Engineering Physics and Mathematics Division

\title{
ENGINEERING PHYSICS AND MATHEMATICS DIVISION PROGRESS REPORT \\ FOR PERIOD ENDING DECEMBER 31, 1992
}

\author{
R. C. Ward, Director
}

Date Published - May 1993

Prepared by the

OAK RIDGE NATIONAL LABORATORY

Oak Ridge, Tennessee 37831 managed by

MARTIN MARIETTA ENERGY SYSTEMS, INC.

for the

U.S. DEPARTMENT OF ENERGY

under contract DE-AC05-84OR21400 
Reports previously issued in this series are as follows:

ORNL-2081
ORNL-2389
ORNL-2609
ORNL-2842
ORNL-3016
ORNL-3193
ORNL-3360
ORNL-3499, Vols. I and II
ORNL-3714, Vols. I and II
ORNL-3858, Vols. I and II
ORNL-3973, Vols. I and II
ORNL-4134
ORNL-4280
ORNL-4433
ORNL-4592
ORNL-4705
ORNL-4800
ORNL-4902
ORNL-4997
ORNL-5101
ORNL-5280
ORNL-5504
ORNL-5725
ORNL-5897, Vols. I and II
ORNL-6030
ORNL-6214
ORNL-6425
ORNL-6582
ORNL-6682

Period Ending September 10, 1956 Period Ending September 1, 1957 Period Ending September 1, 1958 Period Ending September 1, 1959 Period Ending September 1, 1960 Period Ending September 1, 1961 Period Ending September 1, 1962 Period Ending August 1, 1963 Period Ending August 1, 1964 Period Ending August 1, 1965 Period Ending May 31, 1966 Period Ending May 31, 1967 Period Ending May 31, 1968 Period Ending May 31, 1969 Period Ending May 31, 1970 Period Ending May 31, 1971 Period Ending May 31, 1972 Period Ending May 31, 1973 Period Ending August 31, 1974 Period Ending October 31, 1975 Period Ending February 28, 1977 Period Ending November 30, 1978 Period Ending November 30, 1980 Period Ending May 31, 1982 Period Ending December 31, 1983 Period Ending June 30, 1985 Period Ending September 30, 1987 Period Ending August 31, 1989 Period Ending March 31, 1991 
Progress reports for research in the Mathematical Sciences prior to 1984 are as follows:

ORNL-2283

ORNL-2562

ORNL-2915

ORNL-3082

ORNL-3264

ORNL-3423

ORNL-3567

ORNL-3766

ORNL-3919

ORNL-4083

ORNL-4236

ORNL-4385

ORNL-4514

ORNL-4661

ORNL-4761

ORNL-4851

ORNL-4989

UCCND/CSD-18

ORNL/CSD-13

ORNL/CSD-27

ORNL/CSD-34

ORNL/CSD-40

ORNL/CSD-61

ORNL/CSD-82

ORNL/CSD-105

ORNL/CSD-118
Period Ending February 28, 1957

Period Enging August, 31, 1958

Period Ending December 31, 1959

Period Ending December 31, 1960

Period Ending January 31, 1962

Period Ending December 31, 1962

Period Ending December 31, 1963

Period Ending December 31, 1964

Period Ending December 31, 1965

Period Ending December 31, 1966

Period Ending December 31, 1967

Period Ending December 31, 1968

Period Ending December 31, 1969

Period Ending December 31, 1970

Period Ending December 31, 1971

Period Ending December 31, 1972

Period Ending June 30, 1974

Period Ending June 30, 1975

Period Ending June 30, 1973

Period Ending June 30, 1977

Period Ending June 30, 1978

Period Ending June 30, 1979

Period Ending June 30, 1980

Period Ending June 30, 1981

Period Ending June 30, 1982

Period Ending June 30, 1983 


\section{PREFACE}

The primary purpose of this report is to provide an archival record of the research activities of the Engineering Physics and Mathematics Division during the period April 1, 1991, through December 31, 1992. Earlier reports in this series are identified on the previous pages, along with the progress reports describing ORNL's research in the mathematical sciences prior to 1984 when those activities moved into the division.

Division staff have been active participants during the reporting period in defining, planning, and implementing the Department of Energy's (DOE) role in four Presidential Initiatives: Global Change, High Performance Computing and Communications, Biotechnology, and Advanced Manufacturing. Strong emphasis on these national issues will be evident in many of our research programs, e.g., modeling of physical and biological phenomena, scientific computing, informatics, and robotics.

In addition, considerable effort has been extended in laying the groundwork for transferring technology developed in our division to the industrial sector when appropriate. Two Cooperative Research and Development Agreements (CRADA) have been signed and are active, one with Intel's Supercomputer Systems Division on interactive scientific visualization and one with International Business Machines to evaluate a new architecture for parallel computation. Over a dozen more are currently in various stages of the approval and funding process.

Two major administrative decisions significantly affected our division's activities during the reporting period and will continue to do so in the future. First, the DOE's Office of Nuclear Energy decided to shut down the Tower Shielding Facility (TSF). The TSF has been an excellent experimental facility to complement our theoretical and computational studies in reactor design and shielding. That experimental component of our research program will be sorely missed by division and ORNL researchers and by the international shielding community. Second, ORNL created the Center for Computational Sciences (CCS) to manage its High Performance Computing and Communications (HPCC) activities. This organizational change resulted from the laboratory's successful competition for a DOE High Performance Computing Research Center, which was awarded to ORNL in December, 1991. Division management and researchers were heavily involved in developing the proposal and in securing the award. They will continue to play an extremely important role in these activities and now work with CCS management in helping to fulfill the goals and objectives of ORNL's HPCC program.

As in previous reports, our research is described through abstracts of journal articles, technical reports, and presentations organized into sections following the five major operating units in the division: Mathematical Sciences, Intelligent Systems, Nuclear Data and Measurement Analysis, Nuclear Analysis and Shielding, and the Engineering Physics Information Centers. Each section begins with an introduction highlighting honors, awards, and significant research accomplishments in that unit during the reporting period. Some of these items deserve special 
note. GRAIL, a multi-sensor/neural network system for locating genes in genome sequences developed by Ed Uberbacher and Reinhold Mann in our division and Richard Mural in Biology Division, won one of the prestigious and highly competitive 1992 R\&D 100 Awards; JASPER, the joint Japanese-American shielding research program, was completed in 1992; Parallel Virtual Machine (PVM), software developed under the leadership of Al Geist and Jack Dongarra enabling networked computers to function as a single parallel computer, has fast become the de facto industry standard and continues to win awards; in October, 1992, the Oak Ridge Electron Linear Accelerator surpassed 100,000 hours of operation - and without a major work-related injury; and Radiation Shielding Information Center (RSIC) celebrated 30 years of serving the nuclear community in 1992. Information on these accomplishments as well as many others are in the introductions.

Following the abstracts, summary lists of publications and presentations, staff additions and departures, scientific and professional activities of division staff, division seminars, and technical conferences organized and sponsored by our division are included as appendices. Our report concludes with the division's organization chart, as of December 31, 1992, and an author index. 


\section{TABLE OF CONTENTS}

PREFACE

Section 1. MATHEMATICAL SCIENCES

1.0. Introduction . . . . . . . . . . . . . . . . . 3

\section{COMPUTER SCIENCE}

\section{General Parallel Computing}

1.1. A User's Guide to PVM Parallel Virtual Machine

A. Beguelin, J. J. Dongarra, G. A. Geist, R. Manchek, and

V. Sunderam

1.2. Graphical Development Tools for Network-Based Concurrent Supercomputing

A. Beguelin, J. J. Dongarra, G. A. Geist, R. Manchek, and

V. Sunderam

1.3. HeNCE: Graphical Development Tools for Network-Based Concurrent Computing

A. Beguelin, J. J. Dongarra, G. A. Geist, R. Manchek, K. Moore,

R. Wade, and V. Sunderam . . . . . . . . . . . . . . 5

1.4. Heterogeneous Network Computing

A. Beguelin, J. J. Dongarra, G. A. Geist, R. Manchek, and V. Sunderam . . . . . . . . . . . . . . . . 6

1.5. Speedup Properties of Phases in the Execution Profile of Distributed Parallel Programs

B. M. Carlson, T. D. Wagner, L. W. Dowdy, and P. H. Worley . . . . 6

1.6. Communication Performance of the Intel Touchstone Delta Mesh T. H. Dunigan . . . . . . . . . . . . . . . . . . . . . . . . . 6

1.7. Hypercube Clock Synchronization T. H. Dunigan . . . . . . . . . . . . . . . . . . . . . 7

1.8. Kendall Square Multiprocessor: Early Experiences and Performance T. H. Dunigan . . . . . . . . . . . . . . . . . . . . . 7 
1.9. Performance of the Intel iPSC/860 and Ncube 6400 Hypercubes

T. H. Dunigan . . . . . . . . . . . . . . . . . . . . . . . . . 7

1.10. Parallel Superconductor Code Exceeds 2.5 Gflops on iPSC $/ 860$

G. A. Geist, B. Ginatempo. W. A. Shelton, and G. M. Stocks . . . . . 7

1.11. Solving Material Science Problems at OR: L's Center for Computational Studies

G. A. Geist . . . . . . . . . . . . . . . . . . . . . . . . . . . 8

1.12. The PVM System: Supercomputer Level Concurrent Computation on a Heterogeneous Network of Workstations

G. A. Geist and V. S. Sunderam . . . . . . . . . . . . . . . . . . 8

1.13. Network-Based Concurrent Computing on the PVM System

G. A. Geist and V. S. Sunderam . . . . . . . . . . . . . . . . . . 8

1.14. Visualizing the Performance of Parallel Programs

M. T. Heath and J. A. Etheridge . . . . . . . . . . . . . . . . . . 8

1.15. Parallel Computers Take Lead in Complex Problem Solving

M. R. Leuze and R. C. Ward . . . . . . . . . . . . . . . . . . . . 9

1.16. Developing Large Scale Applications by Integrating PVM and the Intel iPS $/ 860$

W. A. Shelton and G. A. Geist . . . . . . . . . . . . . . . . . . . 9

1.17. FERMI Surfaces of $\beta$-Phase NiAl Alloys

G. M. Stocks, W. A. Shelton, D. M. Nicholson, G. A. Geist, and

F. J. Pinski

1.18. The PVM System: Supercomputer Level Concurrent Computation on a Network of IBM RS/6000 Powerstations V. S. Sunderam and G. A. Geist . . . . . . . . . . . . . . . . . . 10

1.19. Standards for Message-Passing in a Distributed Memory Environment D. W. Walker . . . . . . . . . . . . . . . . . . 10

1.20. A New PICL Trace File Format P. H. Worley . . . . . . . . . . . . . . . . . . . . . . . 10

1.21. Phase Modeling of a Parallel Scientific Code P. H. Worley . . . . . . . . . . . . . . . . . . . . . . . . . . . 11 
1.22. PICL: Past, Present, and Future

P. H. Worley . . . . . . . . . . . . . . . . . . . . . 11

1.23. The Effect of Multiprocessor Radius on Scaling

P. H. Worley . . . . . . . . . . . . . . . . . . . . . . . . . 11

\section{Dense Matrix Computations}

1.24. The Design of Scalable Software Libraries for Distributed Memory Concurrent Computers

J. Choi, J. J. Dongarra, and D. W. Walker . . . . . . . . . . . 12

1.25. Level 3 BLAS for Distributed Memory Concurrent Computers

J. Choi, J. J. Dongarra, and D. W. Walker . . . . . . . . . . . . . 12

1.26. ScaLPACK: A Scalable Linear Algebra Library for Distributed Memory Concurrent Computers

J. Choi, J. J. Dongarra, D. W. Walker, and R. Pozo . . . . . . . . . 12

1.27. Numerical Considerations in Computing Invariant Subspaces J. J. Dongarra, S. Hammarling, and J. H. Wilkinson . . . . . . . . 13

1.28. The IBM RISC System/6000 and Linear Algebra Operations J. J. Dongarra, P. Mays, and G. R. di Brozolo . . . . . . . . . . . . 13

1.29. NA-NET Numerical Analysis Net J. J. Dongarra and B. Rosener . . . . . . . . . . . . . . . . . . . 13

1.30. A Parallel Algorithm for the Non-Symmetric Eigenvalue Problem J. J. Dongarra and M. Sidani . . . . . . . . . . . . . . . . . . 14

1.31. Reduction to Condensed Form for the Eigenvalue Problem on Distributed Memory Architectures J. J. Dongarra and R. A. van de Geijn . . . . . . . . . . . . . . . 14

1.32. A Look at Scalable Dense Linear Algebra Libraries J. J. Dongarra, R. A. van de Geijn, and D. W. Walker . . . . . . . . 14

1.33. A Divide and Conquer Approach to the Nonsymmetric Eigenvalue Problem E. R. Jessup . . . . . . . . . . . . . . . . . . . 14 
1.34. A Case Against a Divide Conquer Approach to the Nonsymmetric Eigenvalue Problem

E. R. Jessup .

1.35. Experiments with an Ordinary Differential Equation Solver in the Parallel Solution of Method of Lines Problems on a Shared Memory Parallel Computer, Part II

D. K. Kahaner, E. G. Ng, W. E. Schiesser, and S. Thompson . . . . . 15

1.36. Parallelizing Across Time When Solving Time-Dependent Partial Differential Equations P. H. Worley . . . . . . . . . . . . . . . . . . . . . . . . 16

\section{Sparse Matrix Computations}

1.37. An Introduction to Chordal Graphs and Clique Trees

J. R. S. Blair and B. W. Peyton . . . . . . . . . . . . . . . . . . 16

1.38. On Finding Minimum-Diameter Clique Trees

J. R. S. Blair and B. W. Peyton . . . . . . . . . . . . . . . . . . 16

1.39. Predicting Structure in Nonsymmetric Sparse Matrix Factorizations

J. R. Gilbert and E. G. Ng . . . . . . . . . . . . . . . . . . . . . 17

1.40. An Efficient Algorithm to Compute Row and Column Counts for Sparse Cholesky Factorization

J. R. Gilbert, E. G. Ng, and B. W. Peyton . . . . . . . . . . . . . . 17

1.41. Supernodal Symbolic Cholesky Factorization of a

Local-Memory Multiprocessor E. $N g$. . . . . . . . . . . . . . . . . . . . . . 17

1.42. A Supernodal Cholesky Factorization Algorithm for Shared-Memory Multiprocessors E. G. Ng and B. W. Peyton . . . . . . . . . . . . . . . 18

1.43. A Tight and Explicit Representation of $Q$ in Sparse QR Factorization E. G. Ng and B. W. Peyton . . . . . . . . . . . . . . . . 18

1.44. Block Sparse Cholesky Algorithms on Advanced Uniprocessor Computers E. G. Ng and B. W. Peyton . . . . . . . . . . . . . . . . . 18 
1.45. Partitioning a Chordal Graph Into Transitive Subgraphs for Parallel Sparse Triangular Solution

B. W. Peyton, A. Pothen, and X. Yuan . . . . . . . . . . . . . . . 19

1.46. Parallel Sparse Cholesky Factorization Algorithms for

Shared-Memory Multiprocessor Systems

E. Rothberg, A. Gupta, E. Ng, and B. W. Peyton . . . . . . . . . . . 19

\section{MATHEMATICS}

\section{Applied Mathematics}

1.47. Preconditioned Conjugate Gradient Methods for the Incompressible Navier-Stokes Equations

P. Chin, E. F. D'Azevedo, P. A. Forsyth, and W.-P. Tang . . . . . . . 20

1.48. Drop Tolerance Preconditioning for Incompressible Viscous Flow

E. F. D'Azevedo, P. A. Forsyth, and W.-P. Tang . . . . . . . . . . . 20

1.49. Hypersingular Integral Evaluation Using Maple

L. J. Gray . . . . . . . . . . . . . . . . . . . . . . . . . . . . 20

1.50. Boundary Element Analysis at Oak Ridge National Laboratory

L. J. Gray . . . . . . . . . . . . . . . . . . . . . . . . . . . . 20

1.51. Hypersingular Integral Formulation of Elastic Wave Scattering

L. J. Gray and S. J. Chang . . . . . . . . . . . . . . . . . . . . 21

1.52. Hypersingular Integral Equations at a Corner

L. J. Gray and L. L. Manne . . . . . . . . . . . . . . . . . . . . 21

1.53. Hermite Interpolation Algorithm for Hypersingular Boundary Integrals

L. J. Gray and C. San Soucie . . . . . . . . . . . . . . . . . . . 21

1.54. Optimal Control for Parabolic Systems with Competitive Interactions

S. M. Lenhart and V. Protopopescu . . . . . . . . . . . . . . . . . 21

1.55. A Two-Sided Game for Non Local Competitive Systems with Control on Source Terms

S. M. Lenhart, V. Protopopescu, and S. Stojanovic . . . . . . . . . . 22

1.56. An Overview of Integration Methods for Hypersingular Boundary Integrals

E. Lutz, L. J. Gray, and A. R. Ingraffea . . . . . . . . . . . . . . . 22 
1.57. Indirect Evaluation of Surface Stress in the Boundary Element Method E. Lutz, L. J. Gray, and A. R. Ingraffea . . . . . . . . . . . . . . . 22

1.58. The Performance of a Fluid Code on Massively Parallel Machines V. E. Lynch, B. A. Carreras, J. B. Drake, J. N. Leboeuf, P. Liewer, and D. W. Walker . . . . . . . . . . . . . . . . . . 22

1.59. Three-Dimensional Fracture Simulation with a Single-Domain, Direct Boundary Element Formulation L. F. Martha, A. R. Ingraffea, and L. J. Gray . . . . . . . . . . . . 23

1.60. Simulation of 3D Non-Planar Fatigue Crack Growth in a Turbine Blade Root D. O. Potyondy, L. J. Gray, and A. Ingraffea . . . . . . . . . . . . 23

1.61. Latent Heat Thermal Energy Storage for Lunar Oxygen Production

A. D. Solomon, V. Alexiades, G. Jacobs, M. Naney, and M. Olszewski . . 24

1.62. A Preliminary Study of the Controls on Melting During In Situ Vitrification

A. D. Solomon, J. E. Nyquist, V. Alexiades, G. K. Jacobs, and S. M. Lenhart . . . . . . . . . . . . . . . . . . . . . . . . . . 24

\section{Numerical Analysis}

1.63. Towards a Cost-Effective ILU Preconditioner with High Level Fill E. F. D'Azevedo, P. A. Forsyth, and W.P. Tang . . . . . . . . . . . 25

1.64. Reducing Communication Costs in the Conjugate Gradient Algorithm on Distributed Memory Multiprocessors E. F. D'Azevedo and C. H. Romine . . . . . . . . . . . . . . . . . 25

1.65. Comparison of Iterative Methods for Nonsymmetric Coupled Elliptic Equations J. M. Donato . . . . . . . . . . . . . . . . . . . . . . . . . . . 25

1.66. Iterative Methods for Nonsymmetric Coupled Systems J. M. Donato . . . . . . . . . . . . . . . . . . . . . . . . . . . 26

1.67. The Fourier Analysis Technique and $\epsilon$-Pseudo-Eigenvalues J. M. Donato . 26 
1.68. The Relationship Between $\epsilon$-Pseudo-Eigenvalues and the Fourier Analysis Techniques

J. M. Donato

1.69. Boundary Elements on Distributed Memory Architectures

R. E. Flanery, J. B. Drake, and L. J. Gray . . . . . . . . . . . . . 27

1.70. Two Variants of Minimum Discarded Fill Ordering

P. A. Forsyth, W.-P. Tang, and E. F. D'Azevedo . . . . . . . . . . . 27

1.71. An Implementation of a Parallel MOL Solver on the Intel Gamma

Parallel Computer

W. F. Lawkins and J. S. Payne. . . . . . . . . . . . . . . . . . . 28

1.72. The Hierarchical Spatial Decomposition of Three-Dimensional

Particle-in-Cell Plasma Simulations on MIMD Distributed

Memory Multiprocessors

D. W. Walker . . . . . . . . . . . . . . . . 28

1.73. A FORTRAN 90 Code for Magnetohydrodynamics, Part I:

Banded Convolution

D. W. Walker . . . . . . . . . . . . . . . . . . . . . . 29

\section{Global Climate Modeling}

1.74. Atmospheric Spectral Models on MIMD and SIMD Architectures

J. B. Drake, D. W. Walker, and P. H. Worley . . . . . . . . . . . . 29

1.75. Parallel Algorithms for the Spectral Transform and Semi-Lagrangian Transport Methods

J. B. Drake, D. L. Williamson, and I. Foster . . . . . . . . . . . . . 29

1.76. Parallelizing the Spectral Transform Method - Part II D. W. Walker, P. H. Worley, and J. B. Drake . . . . . . . . . . . . 30

1.77. A Standard Test Set for Numerical Approximations to the Shallow Water Equations in Spherical Geometry

D. L. Williamson, J. B. Drake, J. J. Hack, R. Jakob, and

P. N. Swarztrauber . . . . . . . . . . . . . . . . . . . . . . 31

1.78. Parallelizing the Spectral Transform Method P. H. Worley and J. B. Drake . . . . . . . . . . . . . . . . . . . 31 
1.79. A Mathematical Model for Radiation-Induced Myelopoiesis

T. D. Jones, M. D. Morris, and R. W. Young . . . . . . . . . . . . 31

\section{STATISTICS}

\section{Design and Analysis of Computational Experiments}

1.80. Bayesian Design and Analysis of Computer Experiments: Two Examples T. J. Mitchell and M. D. Morris . . . . . . . . . . . . . . . . . . 32

1.81. Using Computer Experiments to Construct a Cheap Substitute for an Expensive Simulation Model T. J. Mitchell ard M. D. Morris . . . . . . . . . . . . . . . . . . 32

1.82. The Spatial Correlation Function Approach to Response Surface Estimation T. J. Mitchell and M. D. Morris . . . . . . . . . . . . . . . 33

1.83. Exploratory Designs for Computational Experiments M. D. Morris and T. J. Mitchell . . . . . . . . . . . . . . . . . . 33

1.84. Bayesian Design and Analysis of Computer Experiments: Use of Derivatives in Surface Prediction M. D. Morris, T. J. Mitchell, and D. Ylvisaker . . . . . . . . 34

\section{Computational Distribution Theory}

1.85. The Negative Hypergeometric Distribution and Estimation by Moments K. O. Bowman, M. A. Kastenbaum, and L. R. Shenton. . . . . . . . 34

1.86. The Use of Moment Estimators to Determine the Parameters of Concentration Fluctuations in Random Area Atom Probe Analyses K. O. Bowman, M. K. Miller, and L. R. Shenton . . . . . . . . . . . 34

1.87. Parameter Estimation for the Beta Distribution K. O. Bowman and L. R. Shenton . . . . . . . . . . . . . . . . . 35

1.88. Mixtures of Logarithmic Distributions K. O. Bowman, L. R. Shenton, and M. A. Kastenbaum . . . . . . . 35 


\section{Research in General Statistical Methods}

1.89. Overdispersion: Notes on Discrete Distributions

K. O. Bowman, L. R. Shenton, and M. A. Kastenbaum . . . . . . . . 35

1.90. Discrete Pearson Distributions

K. O. Bowman, L. R. Shenton, and M. A. Kastenbaum . . . . . . . . 36

1.91. Asymptotically Valid Prediction Intervals for Linear Models

R. L. Schmoyer . . . . . . . . . . . . . . . . . . . . . . . . . . 36

1.92. Overdispersion of Aggregated Genetic Data

K. O. Bowman and M. A. Kastenbaum . . . . . . . . . . . . . . . 36

1.93. Software Considerations for Reliability Centered Maintenance Analyses

D. M. Flanagan . . . . . . . . . . . . . . . . . 37

1.94. Comparison of Models for Deconvoluting the Compositions of Coexisting Phases

M. K. Miller, K. O. Bowman, A. Cerezo, and J. M. Hyde . . . . . . . 37

1.95. HModel: An X Tool for Global Model Search

G. Ostrouchov . . . . . . . . . . . . . . . . . . . . . . . . 37

\section{Statistical Sampling Theory}

1.96. Statistical Design for Sampling and Analysis of Animal Populations for Chemical Contamination J. J. Beauchamp, J. F. McCarthy, D. Rosenblatt, and L. R. Shugart . . 38

1.97. A Study of Interstate Motor Carrier Vehicle Miles of Travel P. S. Hu, T. Wright, S.-P. Miaou, R. Gorman, and S. C. Davis . . . . 38

1.98. National Profile on Jommercially Generated Low-Level Radioactive Mixed Waste

J. A. Klein, J. E. Mrochek, R. L. Jolley, I. W. Osborne-Lee,

A. A. Francis, and T. Wright . . . . . . . . . . . . . . . . . . . 38

1.99. A Note on Sampling to Locate Rare Defectives with Strong Prior Evidence T. Wright . . . . . . . . . . . . . . . . . . . . . 39 
$\mathrm{xvi}$

\section{Applications of Statistical Methods}

1.100. Selection of Factors Affecting the Presence of Brooktrout in Adirondack Lakes: A Case Study

J. J. Beauchamp, S. W. Christensen, and E. P. Smith . . . . . . . . . 39

1.101. Modeling Speedup in Parallel Sparse Matrix Factorization

M. T. Heath, L. S. Ostrouchov, G. Ostrouchov, and C. H. Romine . . . 40

1.102. Concentration Dependent Protection Against X-Ray Induced

Chromosome Aberrations in Human Lymphocytes by the Aminothiol

WR-1065

L. G. Littlefield, E. E. Joiner, S. P. Colyer, F. Sallam, and

E. L. Frome . . . . . . . . . . . . . . . . . . . . . . . . . . 40

1.103. Experience with the 'Cytochalasin-B Method' for Quantifying

Radiation-Induced Micronuclei in Human Lymphocytes

L. G. Littlefield, A. M. Sayer, F. Sallam, E. L. Frome, and

R. A. Kleinerman. . . . . . . . . . . . . . . . . . . . 41

1.104. Relationship Between Truck Accidents and Highway Geometric Design:

A Poisson Regression Approach

S.P. Miaou, P. S. Hu, T. Wright, A. K. Rathi, and S. C. Davis . . . . 41

1.105. Marrow Cell Kinetics Model: Equivalent Prompt Dose Approximations for Two Special Cases

M. D. Morris and T. D. Jones . . . . . . . . . . . . . . . . . . . 42

1.106. A Cell Kinetics Model of Radiation - Induced Myelopoiesis: Rate

Coefficient Estimates for Mouse, Rat, Sheep, Swine, Dog, and Burro

Irradiated by Photons

M. D. Morris, T. D. Jones, and R. W. Young . . . . . . . . . . . . 42

1.107. Computer Communication: What's Your E-Mail Address?

G. Ostrouchov . . . . . . . . . . . . . . . . . . . . . . . . . .42

1.108. Computer Communication: Resource Discovery

G. Ostrouchov . . . . . . . . . . . . . . . . . . . . . . . 42

1.109. Computer Communication: Alternate Connections and White Pages

G. Ostrouchov . . . . . . . . . . . . . . . . . . . . . 43 
1.110. Using Weibull Statistics to Analyze Ion Beam Enhanced Adhesion as Measured by the Pull Test

J. E. Pawel, W. E. Lever, D. J. Downing, C. J. McHargue,

L. J. Romana, and J. J. Wert . . . . . . . . . . . . . . . . . 43

1.111. Job Factors, Radiation and Cancer Mortality at Oak Ridge

National Laboratory: Follow-Up Through 1984

S. Wing, C. M. Shy, J. L. Wood, S. Wolf, D. L. Cragle,

W. Tankersley, and E. L. Frome

1.112. Assessment of En Route Sector and Terminal Air Traffic Control

Performance

T. A. Vineyard, D. J. Downing, R. C. Durfee, J. J. Edwards,

D. M. Flanagan, M. C. Fletcher, R. T. Goeltz, G. Ostrouchov,

J. A. Rome, M. J. Saltmarsh, J. L. Smyre, and W. R. Wing . . . . . . 44

Section 2. NUCLEAR DATA MEASUREMENT AND EVALUATION

2.0. Introduction . . . . . . . . . . . . . . . . . . 47

\section{EXPERIMENTAL RESULTS AND THEIR ANALYSIS}

2.1. Experimental and Calculated Excitation Functions for Discrete-Line

Gamma-Ray Production Due to $1-40 \mathrm{MeV}$ Neutron Interactions with ${ }^{56} \mathrm{Fe}$

J. K. Dickens, C. Y. Fu, D. M. Hetrick, D. C. Larson, and

J. H. Todd .

2.2. Measurements and Calculations of Radionuclides Created by ${ }^{181} \mathrm{Ta}(\gamma, x n y p)$ Reactions for Bremsstratlung Photons Produced by $150-\mathrm{MeV}$ Electrons

J. K. Dickens and M. A. Miller. . . . . . . . . . . . . . . . . . 50

2.3. Measurements of ${ }^{181} \mathrm{Ta}(\gamma, x n y p)$ Reactions for Bremsstrahlung

Photons Produced by $150-\mathrm{MeV}$ Electrons

J. K. Dickens and M. A. Miller. . . . . . . . . . . . . . . . . . . 50

2.4. Measurement of the Nitrogen Total Cross Section from $0.5 \mathrm{eV}$

to $50 \mathrm{MeV}$ and Analysis of the 433-keV Rosonance

J. A. Harvey, N. W. Hill, and N. M. Larson . . . . . . . . . . . . . 51

2.5. Neutron Physics at the Oak Ridge National Laboratory

J. A. Harvey 
2.6. Review of Fission Product Capture Measurements at the Oak Ridge Electron Linear Accelerator (ORELA)

D. C. Larson and R. L. Macklin 52

2.7. White Source Gamma-Ray-Production Spectral Measurement Facilities in the U.S.

D. C. Larson, J. K. Dickens, R. O. Nelson, and S. A. Wender . . . . . 52

2.8. Study of Radionuclides Created by ${ }^{181} \mathrm{Ta}(\gamma, x n y p)$ Reactions for Bremsstrahlung Photons Produced by $150-\mathrm{MeV}$ Electrons M. A. Miller and J. K. Dickens . . . . . . . . . . . . . . . . . . . 52

2.9. Progress and Problems in Energy-Related Nuclear Data R. W. Peelle . . . . . . . . . . . . . . . . . . 53

2.10. ${ }^{58} \mathrm{Ni}+n$ Transmission, Differential Elastic $\subseteq$ :attering and Capture Measurements and Analysis up to $813 \mathrm{keV}$

C. M. Perey, F. G. Perey, J. A. Harvey, N. W. Hill, N. M. Larson, R. L. Macklin, and D. C. Larson . . . . . . . . . . . . . . . . . 53

2.11. ${ }^{56} \mathrm{Fe}$ and ${ }^{60} \mathrm{Ni}$ Resonance Parameters C. M. Perey, F. G. Perey, J. A. Harvey, N. W. Hill, and N. M. Larson. . . . . . . . . . . . . . . . . . . . . . . . . . . 54

2.12. Electric Properties of the Neutron from Precision Cross Section Measurements J. Schmiedmayer, P. Riehs, J. A. Harvey, and N. W. Hill . . . . . . . 54

2.13. ${ }^{10} \mathrm{~B}\left(n, \alpha_{1} \gamma\right){ }^{7} \mathrm{Li}$ Cross Section Between 0.3 and $4.0 \mathrm{MeV}$ R. A. Schrack, O. A. Wesson, D. C. Larson, J. K. Dickens, and J. H. Todd . . . . . . . . . . . . . . . . . . . . . . . . . . . . 54

2.14. Measurement of the ${ }^{10} \mathrm{~B}(\mathrm{n}, \alpha \gamma)$ Cross Section in the $0.3-$ to $4-\mathrm{MeV}$ Neutron Energy Interval R. A. Schrack, O. A. Wasson, D. C. Larson, J. K. Dickens, and J. H. Todd . . . . . . . . . . . . . . . . . . . . . 55

2.15. Fast Neutron-Induced Changes in Net Impurity Concentration of High-Resistivity Silicon I. Tsveybak, W. Bugg, J. A. Harvey, and J. Walter . . . . . . . . . . 55

2.16. Is the Problem with Pu-239 Fission Cross Section Solved? L. W. Weston, J. A. Harvey, and H. Derrien . . . . . . . . . . . . 55 
2.17. High Resolution Fission Cross Section Measurements of ${ }^{235} \mathrm{U}$ and ${ }^{239} \mathrm{Pu}$ L. W. Weston and J. H. Todd . . . . . . . . . . . . . . . . . . . 56

2.18. R-Matrix Analysis of an ORELA Measurement of the $n+{ }^{48} \mathrm{Ca}$ Total Cross Setion from $100 \mathrm{keV}$ to $3.9 \mathrm{MeV}$

R. F. Carlton, C. H. Johnson, and J. A. Harvey . . . . . . . . . . . 56

2.19. R-Matrix Analysis of an ORELA Measurement of the $n+{ }^{208} \mathrm{~Pb}$ 'Total Cross Section from 78 to $1700 \mathrm{keV}$

R. F. Carlton, R. R. Winters, J. A. Harvey, N. W. Hill, C. H. Johnson, and J. Schmiedmayer . . . . . . . . . . . . . . . . 57

2.20. R-Matrix Analysis of Neutron Total Cross Section Data in the $\mathrm{MeV}$ Region

R. F. Carlton, J. A. Harvey, and C. H. Johrson . . . . . . . . . 57

2.21. Multilevel Resonance Analysis of ${ }^{59}$ Co Transmission Measurements G. de Saussure and N. M. Larson . . . . . . . . . . . . . . . . . . 57

2.22. Multilevel Resonance Analysis of ${ }^{59}$ Co Neutron Transmission Measurements

G. de Saussure, N. M. Larson, J. A. Harvey, and N. W. Hill . . . . . . 58

2.23. Multilevel Resonance Analysis of ${ }^{59}$ Co Neutron Transmission Measurements G. de Saussure, N. M. Larson, J. A. Harvey, and N. W. Hill . . . . . . 58

2.24. Equilibrium and Pre-Equilibrium Models for Calculation of Particle Emission Cross Sections and Spectra C. Y. Fu . . . . . . . . . . . . . . . . . . 54

2.25. Cross Section Parameterization in the Resolved Resonance Region N. M. Larson . . . . . . . . . . . . . . . . . . . . . . . . . . . 59

2.26. Calculation of $(\mathrm{n}, \mathrm{x} \gamma)$ Cross Sections Between Threshold and $100 \mathrm{MeV}$ for $\mathrm{Fe}$ and $\mathrm{Pb}$ Isotopes: Comparison with Experimental Data P. G. Young, R. C. Haight, R. O. Nelson, S. A. Wender, C. M. Laymon, G. L. Morgan, D. M. Drake, M. Drosg, H. Vonach, A. Pavlik, S. Tagesen, D. C. Larson, and D. S. Dale . . . . . . . . . . . . . 59

2.27. Theoretical Calculation of Neutron Induced Data of ${ }^{19} \mathrm{~F}$ and Uncertainties of Parameters Z. Zhixiang, C. Y. Fu, and D. C. Larson . . . . . . . . . . . . . . 59 


\section{CROSS SECTION EVALUATION AND NUCLEAR MODELING}

2.28. International Evaluation Cooperation Task 1.1: Intercomparison of Evaluated Files for ${ }^{52} \mathrm{Cr},{ }^{56} \mathrm{Fe}$, and ${ }^{58} \mathrm{Ni}$

C. Y. Fu, D. C. Larson, D. M. Hetrick, H. K. Vonach, J. Kopecky, S. Iijima, N. Yamamuro, and G. Maino . . . . . . . . . . . . . . . 60

2.29. Tools and Techniques for Evaluation Intercomparison D. M. Hetrick

2.30. Approximate Methods for Generation of Covariance Data for the Structural Materials of ENDF/B-VI

D. M. Betrick, D. C. Larson, and C. Y. Fu . . . . . . . . . . . . 61

2.31. Evaluation of the Silicon Isotopes for ENDF/B-VI

D. M. Hetrick, D. C. Larson, N. M. Larson, C. Y. Fu, and

S. J. Epperson . . . . . . . . . . . . . . . . . . . . . . . . . . 61

2.32. NEACRP/NEANDC Working Group on International Evaluation Cooperation

D. C. Larson, C. L. Dunford, and C. Nordborg . . . . . . . . . . . .61

2.33. Potential Improvements to ENDF/B-VI for Fusion Data

D. C. Larson and C. Y. Fu . . . . . . . . . . . . . . . . . . . . . 62

2.34. Covariances as Input to and Output from Resonance Analyses

N. M. Larson. . . . . . . . . . . . . . . . . . . . . . . . . . . 62

2.35. Covariance Methods for Standards Evaluations

R. W. Peelle . . . . . . . . . . . . . . . . . . 62

2.36. The Resolution of Discrepancies Among Nuclear Data

R. W. Peelle . . . . . . . . . . . . . . . . . 62

2.37. Approximate Methods for Derivation of Covariance Data

S. Tagesen and D. C. Larson . . . . . . . . . . . . . . . . . . . .63

2.38. Covariance Matrix of Model Parameters and Uncertainties of Calculated Neutron Cross Sections on ${ }^{19} \mathrm{~F}$ ENDF/B-VI

Z. Zhao, C. Y. Fu and D. C. Larson . . . . . . . . . . . . . . . . 63

2.39. The Covariance Matrix of Derived Quantities and Their Combination Z. Zhao and F. G. Perey . . . . . . . . . . . . . . . . . . . . . . 63 
2.40. Time Dependent Monte Carlo Calculations of the ORELA Target Neutron Spectrum

S. N. Cramer and F. G. Perey . . . . . . . . . . . . . . . . . . .63

2.41. Computed Secondary-Particle Energy Spectra Following Nonelastic

Neutron Interactions with ${ }^{12} \mathrm{C}$ for $E_{n}$ Between 15 and $60 \mathrm{MeV}$ :

Comparisons of Results from Two Calculational Methods

J. K. Dickens . . . . . . . . . . . . . . . . . . . . . . . . . . 64

2.42. Scintillation Detector Efficiencies for Neutrons in the Energy Region Above $20 \mathrm{MeV}$

J. K. Dickens . . . . . . . . . . . . . . . . . . . . . . . . . .64

2.43. Current Status and Proposed Improvements to the ANSI/ANS-5.1

American National Standard for Decay Heat Power in Light

Water Reactors

J. K. Dickens, T. R. England, and R. E. Schenter . . . . . . . . . . 65

2.44. Analyses of Physics Specimens in Fuel Pins 1 and 2 Irradiated in the

Dounreay Prototype Fast Reactor

S. Raman, B. L. Broadhead, J. K. Dickens, R. L. Walker, and

J. L. Botts .

Section 3. INTELLIGENT SYSTEMS

3.0. Introduction . . . . . . . . . . . . . . . . . . 69

\section{ROBOTICS AND INTELLIGENT SYSTEMS}

3.1. CESAR Robotics and Intelligent Systems Research for Nuclear Environments

R. C. Mann . . . . . . . . . . . . . . 75

3.2. Robotics and Intelligent Systems Research at the Oak Ridge National Laboratory

F. G. Pin

3.3. Proceedings of the Sixth International Symposium on Methodologies for Intelligent Systems K. S. Harber . . . . . . . . . . . . . . . . . . . . . . . . . . . 76 
3.4. Research and Development at ORNL/CESAR Towards Cooperating Robotic Systems for Hazardous Environments

R. C. Mann, K. Fujimura, and M. A. Unseren . . . . . . . . . . . . 76

3.5. A New Wheel Control System for the Omnidirectional HERMIES-III Robot

D. B. Reister. . . . . . . . . . . . . . . . . . . . . . . . . . . 76

3.6. Time Optimal Trajectories for Mobile Robots with Two IndependentlyDriven Wheels

D. B. Reister and F. G. Pin . . . . . . . . . . . . . . . . . . . . 77

3.7. A System for Simulating Shared Memory in Heterogeneous DistributedMemory Networks with Specialization for Robotics Applications J. P. Jones, A. L. Bangs, and P. L. Butler . . . . . . . . . . . . . . 77

3.8. Hetero Helix: Synchronous and Asynchronous Control Systems in Heterogeneous Distributed Networks J. P. Jones, P. L. Butler, S. E. Johnston, and T. E. Heywood . . . . . 78

3.9. A Concurrent Control Architecture for Autonomous Mobile Robots Using Asynchronous Production Systems

S. S. Iyengar, F. G. Pin, J. Graham, V. G. Hedge, and P. Graham . . . 78

3.10. Dynamic Coupling Effects in Modeling and Control of Hard Contact Motion of a Manipulator M. A. Unseren . . . . . . . . . . . . . . . . . . . . . . . . . . 78

3.11. Input Relegation Control for Gross Motion of a Kinematically Redundant Manipulator M. A. Unseren . . . . . . . . . . . . . . . . . . . . . . . . . . 79

3.12. An Implementation of Redundancy Resolution and Stability Monitoring for a Material Handling Vehicle

A. L. Bangs, F. G. Pin, and S. M. Killough . . . . . . . . . . . . . 79

3.13. Autonomous Surveillance for Surface Radiation Contamination J. P. Jones, P. L. Butler, M. Beckerman, and F. J. Sweeney . . . . . . 79

3.14. DEMO 89 - The Initial Experiment with the HERMIES-III Robot D. B. Reister, J. P. Jones, P. L. Butler, M. Beckerman, and F. J. Sweeney 
3.15. Design of an Omnidirectional and Holonomic Wheeled Platform Prototype

S. M. Killough and F. G. Pin . . . . . . . . . . . . . . . . . . . 80

3.16. Mobile Manipulator Configuration Optimization Using Evolutionary

Programming

J. R. McDonnell, B. L. Andersen, W. C. Page, and F. G. Pin . . . . 80

3.17. A Model of Reactive Planning for Multiple Mobile Agents

K. Fujimura . . . . . . . . . . . . . . . . 81

3.18. Motion Planning in Dynamic Environments

K. Fujimura . . . . . . . . . . . . . . . . . . 81

3.19. On Motion Planning Amidst Transient Obstacles

K. Fujimura

3.20. Navigation of a Car-Like Mobile Robot Using a Decomposition of the Environment in Convex Cells

H. A. Vasseur, F. G. Pin, and J. R. Taylor . . . . . . . . . . . . . 82

3.21. Control of Autonomous Mobile Robots Using Custom-Designed Qualitative Reasoning VLSI Chips and Boards

F. G. Pin and R. S. Pattay . . . . . . . . . . . . . . . . . . . . 83

3.22. Using Custom-Designed VLSI Fuzzy Inferencing for the Autonomous Navigation of a Mobile Robot Chips

F. G. Pin, H. Watanabe, J. Symon, and R. S. Pattay . . . . . . . . . 83

3.23. Merging the Fuzzy and Behaviorist Approaches Using VLSI Fuzzy Inferencing Chips: An Efficient Technique for the Autonomous Navigation of a Mobile Robot F. G. Pin and H. Watanabe

3.24. Autonomous Navigation of a Mobile Robot Using Custom-Designed Qualitative Reasoning VLSI Chips and Boards F. G. Pin, H. Watanabe, J. Symon, and R. S. Pattay . . . . . . . . . 84

3.25. Sensor-Based Driving of a Car with Fuzzy Inferencing VLSI Chips and Boards F. G. Pin and Y. Watanabe 
xxiv

3.26. Implementing Valiant's Learnability Theory Using Random Sets

E. M. Oblou

3.27. N-Learners Problem: Learning Boolean Combination of Halfspaces

N. S. V. Rao, E. M. Oblow, and C. W. Glover . . . . . . . . . . 85

3.28. N-Learners Problem: Fusion of Concepts

N. S. V. Rao, E. M. Oblow, C. W. Glover, and G. E. Liepins . . . . . 85

3.29. Analysis of Complexity Bounds for Pac-Learning with Random Sets

E. M. Oblow and V. R. R. Uppuluri . . . . . . . . . . . . . . . . . 86

3.30. Algorithms for Recognizing Planar Polygonal Configurations Using

Perspective Images

N. S. V. Rao, W. Wu, and C. W. Glover . . . . . . . . . . 86

3.31. Empirical Characterization of a High Intensity LED Proximity Sensor

J. E. Baker . . . . . . . . . . . . . . . . . . . . . . . . 86

3.32. Terrain Following of Arbitrary Surfaces Using a High Intensity LED

Proximity Sensor

J. E. Baker

3.33. Phase I Report on Sensor Technology, Data Fusion and Data

Interpretation for Site Characterization

M. Beckerman . . . . . . . . . . . . . . . . . . . . . . . . . 87

3.34. Laser Range Camera Calibration

K. Storjohann and E. Saltzen. . . . . . . . . . . . . . . . . 87

3.35. Restoration and Fusion of Laser Range Camera Images

M. Beckerman and F. J. Sweeney . . . . . . . . . . . . . . . . . 87

3.36. Sensor Data Fusion for Mobile Robots

M. Beckerman . . . . . . . . . . . . . . . . . . . . . . 88

3.37. A Bayes-Maximum Entropy Method for Multi-Sensor Data Fusion

M. Beckerman . . . . . . . . . . . . . . . . . 88

3.38. Classifier System Learning of Boolean Concepts

G. E. Liepins and L. W. Wang . . . . . . . . . . . . . . . . . . . 88 
3.39. Polynomials, Basis Sets, and Deceptiveness in Genetic Algorithms

G. E. Liepins and M. D. Vose . . . . . . . . . . . . . . . . . . . 89

3.40. Genetic Algorithms and Computerized Trading Strategies

R. J. Bauer, Jr. and G. E. Liepins . . . . . . . . . . . . . . . . . 89

3.41. Schemata Disruption

M. D. Vose and G. E. Liepins . . . . . . . . . . . . . . . . . . . 89

\section{HUMAN FACTORS}

3.42. A Research Profile of the Cognitive Systems and Human Factors Group at the Oak Ridge National Laboratory

H. E. Knee and R. J. Carter . . . . . . . . . . . . . . . . . . . .90

3.43. Individual and Team Communication in a Dynamic Task

M. Terranova, D. Hartley, B. G. Coury, and K. N. Hooper . . . . . . . 90

3.44. Survey of Human Factors Applications of Artificial Neural Networks P. F. Spelt . . . . . . . . . . . . . . . . . . . . . . . . 90

3.45. Introduction to Artificial Neural Networks for Human Factors P. F. Spelt . . . . . . . . . . . . . . . . . . . . . . . 91

3.46. Human Factors and Safety Issues Associated with Actinide Retrieval from Spent Light Water Reactor Fuel Assemblies P. F. Spelt . . . . . . . . . . . . . . . . . . . . . . . . . . 91

3.47. Advanced Control Room Design Review Guidelines: Merging Old and New R. J. Carter and J. A. Wachtel . . . . . . . . . . . . . . . . . . . 92

3.48. Models of Human Operators: Their Need and Usefulness for Improvement of Advanced Control Systems and Control Rooms H. E. Knee and J. C. Schryver . . . . . . . . . . . . . . . . . . .92

3.49. A Training Effectiveness Program for Training Systems R. J. Carter . . . . . . . . . . . . . . . . . . . 92

3.50. On the Design and Development of a Human-Robot Synergistic System F. G. Pin, L. E. Parker, and F. Depiero . . . . . . . . . . . . . . . 93 
xxvi

\section{INFORMATICS}

3.51. GRAIL: An Integrated Artificial Intelligence System for Gene Recognition and Interpretation

X. Guan, R. J. Mural, J. R. Einstein, R. C. Mann, and

E. C. Uberbacher . . . . . . . . . . . . . . . . . . . 93

3.52. An Artificial Intelligence Approach to DNA Sequence Feature Recognition

R. J. Mural, J. R. Einstein, X. Guan, R. C. Mann, and

E. C. Uberbacher . . . . . . . . . . . . . . . 94

3.53. GAP - A Computer Program for Gene Assembly

J. R. Einstein, E. C. Uberbacher, X. Guan, R. J. Mural, and

R. C. Mann . . . . . . . . . . . . . . . . . . . . . 94

3.54. Gene Recognition and Assembly in the GRAIL System: Progress and Challenges

E. C. Uberbacher, J. R. Einstein, X. Guan, and R. J. Mural . . . . . . 94

3.55. Computer-Based Construction of Gene Models Using the GRAIL Gene Assembly Program

J. R. Einstein, R. J. Mural, X. Guan, and E. C. Uberbacher . . . . . . 95

3.56. Combining Neural Networks and Expert Systems to Identify Features in DNA Sequences

R. J. Mural, X. Guan, J. R. Einstein, and E. C. Uberbacher . . . . . . 95

3.57. Searching for Consensus Patterns on a Hypercube Complex Systems

X. Guan, R. C. Mann, R. Mural, and E. C. Uberbacher . . . . . . . . 96

\section{Section 4. NUCLEAR ANALYSIS AND SHIELDING}

4.0. Introduction . . . . . . . . . . . . . . . . . . . . . . . .99

\section{FISSION REACTORS}

4.1. Design Calculations for the ANS Cold Neutron Source II: Heating Rates R. G. Alsmiller, Jr. anl R. A. Lillie . . . . . . . . . . . . . . . 101

4.2. Simulation of the Pulsed Neutron Source Experiment M. Carl and F. C. Difilippo . . . . . . . . . . . . . . . . . 101 
4.3. Application of 3-Dimensional Radiation Transport Codes to the Analysis of the CRBR Prototypic Coolant Pipe Chaseway Neutron Streaming Experiment

K. Chatani

4.4. Cross Sections for Actinide Burner Reactors

F. C. Difilippo

4.5. Effects of the Two-Fission-Neutron Distributions in Reactivity Measurements with the ${ }^{252}$ Cf Source

F. C. Difilippo

4.6. LEU-HTR PROTEUS: Theory and Simulations of Reactivity Measurements with the Inverse Kinetics Method

F. C. Difilippo

4.7. LEU-HTR PROTEUS: Neutron Kinetics and Procedures to Analyze

Pulsed Neutron Experiments

F. C. Difilippo

4.8. PNS Measurements of Effective Graphite Absorption

F. C. Difilippo

4.9. Temperature-Dependent Bondarenko Factors for ${ }^{237} \mathrm{~Np},{ }^{241} \mathrm{Am}$, and ${ }^{243} \mathrm{Am}$

F. C. Difilippo

4.10. Temperature-Dependent Bondarenko Factors for ${ }^{243} \mathrm{Cm},{ }^{244} \mathrm{Cm}$, and ${ }^{245} \mathrm{Cm}$

F. C. Diflippo . . . . . . . . . . . . . . . 103

4.11. Stochastic Processes in Nuclear Reactors Under Parametric Excitation F. C. Difilippo and K. Macko . . . . . . . . . . . . . . . . . . . 104

4.12. Application of a New Integral Representation of the $K_{0}$ Bessel Function

F. C. Difilippo and R. B. Perez . . . . . . . . . . . . . . . . . 104

4.13. Status and Covariance Files of the Scattering Matrix of Graphite F. C. Difilippo, J. P. Renier, and B. A. Worley . . . . . . . . . . . 104

4.14. Framework for Monitoring Reliability M. J. Haire, M. S. Smith, and D. R. Moser . . . . . . . . . . . 105 
4.15. Calculated Energy and Angular Dependence of Particle Fluxes at the Exit of the Advanced Neutron Source Radial and Tangential

Beam Tubes

M. Hayashi, T. Nishigori, R. G. Alsmiller, Jr., and R. A. Lillie . . . 105

4.16. Early Test Facilities and Analysic Methods for Radiation Shielding

D. T. Ingersoll and J. K. Ingersoll

4.17. Phase II Testing of ENDF/B-VI Shielding Data

D. T. Ingersoll, R. Q. Wright, and C. O. Slater

4.18. Needs for Evaluated Covariance Datı for Reactor Pressure Vessel

Dosinetry

B. E. Maerker, B. L. Broadhead, and J. J. Wagschal . . . . . . . . 106

4.19. Reactor Physirs Input to the Safety Analysis Report for the High Flux Isotope Reactor

R. T. Primm, III . . . . . . . . . . . . . . . . . . . . . . 106

4.20. Caiculation of Critical Experiment Parameters for the High Flux Isotope Reactor

R. T. Primm, III . . . . . . . . . . . . . . . . . 107

4.21. Criticality Safety Studies for the Storage of Waste from Nuclear Fuel Services in Intercell Storage Wells 2 and 3 of Building 3019

R. T. Primm, III, C. M. Hopper, and G. R. Smolen . . . . . . . . . 107

4.22. An Assessment of Liquid Metal Centrifugal Pumps at Three Fast Reactors

M. S. Smith, D. H. Wood, and J. D. Drischler . . . . . . . . . . . 107

4.23. System Model Development for Nuclear Thermal Propulsion

J. T. Walton, N. A. Bannan, K. R. Perkins, J. J. Buksa, B. A. Worley, and D. Dobranich . . . . . . . . . . . . . . . . . 108

4.24. Motor-Operated Globe Valve Performance in a Liquid Sodium

Environment

D. H. Wood, M. S. Smitth, and J. D. Drischler . . . . . . . . . . . 108 
$\operatorname{xxix}$

\section{HIGH-ENERGY ACCELERATORS AND DETECTORS}

4.25. Liquid Scintillator Calorimetry (Second Year) a Research and Development Renewal Proposal to the Superconducting Super Collider Laboratory

E. Ables..., M. L. Bauer, B. L. Bishop, H. O. Cohn, T. A. Gabriel,

A. Gordeev, Y. Kamyshkov, R. A. Lillie, F. Plasil, K. Read,

M. J. Rennich, A. Savin, K. Shmakov, B. H. Singeltary, A. Smirnov,

E. Tarkovsky, R. A. Todd, K. G. Young, et al. . . . . . . . . . 109

4.26. HECC92, An Updated Intranuclear Cascade Model of High-Energy Hadron-Nucleus Collisions

F. S. Alsmiller, R. G. Alsmiller, Jr., and O. W. Hermann . . . . . . 109

4.27. The Use of Delay Times in the Intranuclear Cascade Model of High-Energy $(\sim 5 \mathrm{GeV})$ Hadron-Nucleus Collisions

F. S. Alsmiller, R. G. Alsmiller, Jr., and O. W. Hermann . . . . . . 110

4.28. Particle Production Models in HETC 88 in the Energy Range 3 to $30 \mathrm{GeV}$

R. G. Alsmiller, Jr., and F. S. Alsmiller . . . . . . . . . . . . . 110

4.29. The Super Fixed Target Beauty Facility at the SSC

S. Anassontzis, H. Brashear, N. Ericison, T. A. Gabriel,

T. Moore, A. Wintenberg, et al.

4.30. Liquid Argon Calorimeter Design Study Using the CALOR89 Code System

C. Y. Fu, R. G. Alsmiller, Jr., and T. A. Gabriel . . . . . . . . . . 111

4.31. The Computational Challenges Provided by the Codes Used to Design and Analyze Superconducting Super Collider Detectors T. A. Gabriel. . . . . . . . . . . . . . . . . . . . . . . . . . 111

4.32. Comparison of Experimental and Calculational Energy Deposition Profiles in Calorimeters and Other Damage Related Data T. A. Gabriel, T. Handler, and P. K. Job . . . . . . . . . . . . . 112

4.33. Charged Particle Track Reconstruction Using Artificial Neural Networks C. W. Glover, C. Y. Fu, T. A. Gabriel, and T. Handler . . . . . . . 112 
4.34. Hybrid Central Tracking Chamber Collaboration - Progress Report for FY 1991

A. T. Goshaw..., T. A. Gabriel, G. T. Alley, C. L. Britton, Jr.,

C. W. Glover, D. Vandergriff, H. R. Brashear, M. Emery,

T. Ryan, et al.

4.35. Feasibility of Using Neural Networks as a Level 2 Calorimeter Trigger for Jet Tagging

T. Handler, E. Neis, C. W. Glover, T. A. Gabriel, and S. Saini . . . 113

4.36. Comparison of CALOR89 Model Predictions with Scintillator

Plate Calorimeter Data

P. K. Job, L. E. Price, J. Proudfoot, T. Handler, B. L. Bishop, and

T. A. Gabriel.

4.37. Design Considerations for a Scintillating Plate Calorimeter

P. K. Job, L. E. Price, J. Proudfoot, T. Handler, and

T. A. Gabriel. . . . . . . . . . . . . . . . . . . . . . . . . . 113

4.38. Systematic Effe :ts in CALOR Simulation Code to Model

Experimental ćonfigurations

P. K. Job, J. Proudfoot, T. Handler, and T. A. Gabriel . . . . . . .

4.39. Silicon Electromagnetic Calorimeter Collaboration Progress Report

H. W. Kraner..,, G. T. Alley, M. L. Bauer, C. L. Britton, Jr.,

H. R. Brashear, H. O. Cohn, T. A. Gabriel, F. Plasil, M. F. Rennich,

R. A. Todd, et al. . . . . . . . . . . . . . . . . 114

4.40. Radiation Shielding Requirements for Manned Deep Space Missions R. T. Santoro and 7. T. Ingersoll . . . . . . . . . . . . . . . . 114

4.41. Liquid Argon Calorimetry for the SSC Progress Report for FY 91 and Request for Funds for FY 92

R. Steiner..., R. G. Alsmiller, Jr., C. Y. Fu, T. A. Gabriel, et al. . . . 115

4.42. Simulation of a Presampler Response with CALOR - A Comparison with Experimental Data

C. Zeitnitz, G. Lindström, E. Fretwurst, V. Riech, T. A. Gabriel, and T. Handler. . . . . . . . . . . . . . . . . . . . . 115 
$\mathrm{xxxi}$

\section{DEFENSE APPLICATIONS}

4.43. Nuclear Vulnerability of the U.S. M60A1 Tank in an Initial Radiation Environment: MASH Code System Analysis

J. M. Barnes, J. O. Johnson, T. J. Burns, and J. D. Drischler . . . . 116

4.44. Decay of Gamma Rzdiation in Fallout Following a Nuclear War

C. M. Haaland . . . . . . . . . . . . . . . . . . . . . . . . . 116

4.45. Perturbed Environment Assessments for Space Vehic'es

C. M. Haaland . . . . . . . . . . . . . . . . . . . . . . . . . 117

4.46. Calculation of Protection Factors of the M1A1 Tank Against Initial Nuclear Radiation

C. M. Haaland, J. O. Johnson, R. T. Santoro, and K. G. Kerris . . . 117

4.47. Nuclear Vulnerability of the U.S. M60A1 Tank in an Initial

Radiation Environment: MASH Code System Analysis

J. O. Johnson, T. J. Burns, J. D. Drischler, and J. M. Barnes . . . . 117

4.48. Analysis of the Fall-1989 Two-Meter Box Test Bed Experiments

Performed at the Army Pulse Radiation Facility (APRF)

J. O. Johnson, J. D. Drischler, and J. M. Barnes . . . . . . . . . . 118

4.49. Analysis of the Spring- 1990 Two-Meter Box Test Bed Experiments

Performed at the Army Pulse Radiation Facility (APRF)

J. O. Johnson, J. D. Drischler, and J. M. Barnes . . . . . . . . . . 118

4.50. Analysis of the Spring-1991 Two-Meter Box Test Bed Experiments

Performed at the Army Pulse Radiation Facility (APRF)

J. O. Johnson, J. D. Drischler, and J. M. Barnes . . . . . . . . . . 119

4.51. Analytic Methods for Radiation Environment Simulation and

Vulnerability Assessment

J. O. Johnson, R. T. Santoro, T. J. Burns, and R. A. Kehlet . . . . 119

4.52. Radiation Protection for Human Exploration of the Moon and Mars: Application of the MASH Code System

J. O. Johnson, R. T. Santoro, J. D. Drischler, and J. M. Barnes . . . 120

4.53. Radiation Exposure Inside Reinforced Concrete Buildings at Nagasaki

W. A. Rhoades, R. L. Childs, and D. T. Ingersoll . . . . . . . . . . 120 
4.54. Transmission Factors for the Penetration of Neutron and Photon Fluence Into Wood-Frame Dwellings, 1990 (TF90)

W. A. Rhoades, R. A. Lillie, and M. B. Emmett . . . . . . . . . . 121

4.55. DNA Radiation Environments Program Fall 1989 2-Meter Box

Experiments and Analysis

R. T. Santoro

4.56. Resolution of Dose and Reduction Factor Questions in Army Pulse

Radiation Facility Experiments: Renormalization of Calculated and Measured Data

R. T. Santoro, J. D. Drischler, and J. O. Johnson . . . . . . . . . 122

4.57. DNA Radiation Environments Program Spring 1990 2-Meter Box Experiments and Analyses

R. T. Santoro and S. Y. Whitaker . . . . . . . . . . . . . . . . 122

\section{GENERAL METHODS DEVELOPMENT}

4.58. A Comparison Between the Finite Difference and Nodal Integral Methods for the Neutron Diffusion Equation

Y. Y. Azmy

4.59. A Nodal Integral Method for Neutron Diffusion in Hexagonal Geometry Y. Y. Azmy

4.60. Arbitrarily High Order Characteristic Methods for Solving the Neutron Transport Equation

Y. Y. Azmy

4.61. General Order Nodal Transport Methods and Application to Parallel Computing

Y. Y. Azmy

4.62. Performance and Performance Modeling of a Parallel Algorithm for Solving the Neutron Transport Equation

Y. Y. Azmy

4.63. General Order Characteristic Methods for Solving Neutron Transport Problems

Y. Y. Azmy 
4.64. Graphical-Based Construction of Combinatorial Geometries for Radiation Transport and Shielding Applications

T. J. Burns

4.65. CGVIEW - A Program to Generate Isometric and Perspective Views of Combinatorial Geometries

T. J. Burns

4.66. Graphical Debugging of Combinatorial Geometry

T. J. Burns and M. S. Smith

4.67. The Weighted-Difference Form of the Nodal Diffusion Method in Cylindrical Geometry

L. Deleanu, Y. Y. Azmy, and R. T. Primm, III . . . . . . . . . . . 126

4.68. The Cell Analytical-Numerical (CAN) Method for the Solution of the Richards Equation

O. A. Elnawawy and Y. Y. Azmy . . . . . . . . . . . . . . . . . 127

4.69. Parallelization of a Spherical $S_{N}$ Algorithm Based on the Spatial Domain Decomposition

A. Haghighat and Y. Y. Azmy . . . . . . . . . . . . . . . . . . 127

4.70. A User's Manual for MASH 1.0 - A Monte Carlo Adjoint Shielding Code

J. O. Johnson . . . . . . . 128

4.71. DRC2: A Code with Specialized Applications for Coupling Localized Monte Carlo Adjoint Calculations with Fluences from Two-Dimensional R-Z Discrete Ordinates Air-Over-Ground Calculations A User's Manual for MASH 1.0 - A Monte Carlo Adjoint

C. O. Slater.

4.72. DCOR: A Deterministic Combat Model Code Y. Y. Azmy

4.73. Lie Group Analysis for a Multispecies, Spatially Inhomogeneous, Mutually Interacting Gas Mixture

Y. Y. Azmy, V. C. Boffi, V. Protopopescu, and J. Mandrekas . . . . 129

4.74. Sustainability and Profitability in Ecological Systems with Harvesting S. J. Gaff and V. Protopopescu . . . . . . . . . . . . . . . . . . 
xxxiv

4.75. Graphics Development of DCOR: Deterministic Combat Model of Oak Ridge

G. Hunt and Y. Y. Azmy . . . . . . . . . . . . . . . . . . . . 130

4.76. Topological Chaos for a Class of Linear Models

V. Protopopescu and Y. Y. Azmy . . . . . . . . . . . . . . . . . 130

4.77. Analytic Modeling vs Simulation for Classical Warfare

V. Protopopescu, R. T. Santoro, Y. Y. Azmy, and B. L. Kirk . . . . 130

4.78. Combat Modeling with Imprecise Data

V. Protopopescu, R. T. Santoro, Y. Y. Azmy, and B. L. Kirk . . . . 131

SENSITIVITY AND UNCERTAINTY ANALYSIS

4.78. GRESS Version 2.0 User's Manual

J. E. Horwedel . . . . . . . . . . . . . . . . . . . . . . . . . 131

4.80. GRESSing Along

J. E. Horwedel . . . . . . . . . . . . . . . . . . . . . . . . . 131

4.81. An Automated Sensitivity Analysis Procedure Applied to a Dissolved

Oxygen Simulation Model

J. E. Horwedel, S. F. Railsback, and H. I. Jager . . . . . . . . . . 132

4.82. Automated Sensitivity Analysis of an Atmospheric Dispersion Model

J. E. Horwedel, R. J. Raridon, and R. Q. Wright . . . . . . . . . . 132

\section{THEORETICAL ENGINEERING}

4.83. Electric Power Generation by Steady Flow Liquid Metal MHD Generators C. M. Haaland . . . . . . . . . . . . . . . . . . . . . . . . . 133

4.84. Minimum Engine Size for Optimum Automobile Acceleration

C. M. Haaland . . . . . . . . . . . . . . . . . . . . . . . . . 
Section 5. ENGINEERING PHYSICS INFORMATION CENTERS (EPIC)

5.0. Introduction . . . . . . . . . . . . . . . . . . . . . . . . . 137

\section{RADIATION TRANSPORT DEVELOPME?,TS}

5.1. Implementation of the Monte Carlo Charged Particle Transport Code EGS4 on the Hypercube

B. L. Kirk, Y. Y. Azmy, T. A. Gabriel, and C. Y. Fu . . . . . . . . 140

5.2. Analytic Estimation of Radiation Effects in Space Using Solid Angles S. N. Cramer. . . . . . . . . . . . . . . . . . . 140

\section{APPLIED NUCLEAR DATA DEVELOPMENTS}

5.3. New Gamma-Ray Buildup Factor Data for Point Kernel Calculations: ANS-6.4.3 Standard Reference Data D. K. Trubey . . . . . . . . . . . . . . . . . . . . . 140

5.4. Specifications for the Development of BUGLE-93: An ENDF/B-VI Multigroup Cross Section Library for LWR Shielding and Pressure Vessel Dosimetru J. E. White, R. Q. Wright, R. W. Roussin, and D. T. Ingersoll . . . . 141

5.5. International Evaluation Cooperation Subgroup 7: Multigroup Cross Section Processing

R. W. Roussin, J. E. White, E. Sartori, G. Panini, R. MacFarlane, D. Muir, M. Mattes, I. Hasegawa, H. Takano, and F. Mann . . . . . 141

5.6. Transport Calculations of Neutron Transmission Through Steel Using ENDF/B-V, Revised ENDF/B-V and ENDF/B-VI Iron Evaluations

M. L. Williams, C. Aboughantous, M. Asgari, J. E. White, R. Q. Wright, and F. B. K. Kam . . . . . . . . . . . . 141

\section{RADIATION TRANSPORT STUDIES}

5.7. Shielding Design Methods of a Synchrotron Radiation Facility Part I: Accelerator S. Ban and H. Hirayama. 
xxxvi

5.8. Time-Dependent Monte Carlo Calculations of the Oak Ridge Electron Linear Accelerator Target Neutron Spectrum.

S. N. Cramer and F. G. Perey . . . . . . . . . . . . . . . . . . 142

5.9. Investigation of Radiation Effects in Hiroshima and Nagasaki Using a General Monte Carlo-Discrete Ordinates Coupling Scheme

S. N. Cramer and C. O. Slater . . . . . . . . . . . . . . . . . 143

\section{INFORMATION ANALYSIS CENTER ACTIVITIES}

5.10. JANE: A New Information Retrieval System for the Radiation Shielding Information Center

D. K. Trubey . . . . . . . . . . . . . . . . . . . . . . . . . . 143

5.11. Deterministic Methods in Radiation Transport

A. F. Rice and R. W. Roussin . . . . . . . . . . . . . . . . . 143

5.12. Computer Code for Checking, Plotting and Processing of Neutron

Cross-Section Covariance Data and Their Application

E. Sartori and R. W. Roussin . . . . . . . . . . . . . . . . . . 144

\section{APPENDICES}

Personnel Changes . . . . . . . . . . . . . . . . . . . . 147

Scientific and Professional Activities . . . . . . . . . . . . . 153

Conferences . . . . . . . . . . . . . . . . . . . . 181

Seminars at ORNL . . . . . . . . . . . . . . . . 184

Publications . . . . . . . . . . . . . . . . . . . . 190

Papers Presented at Scientific Meetings and Seminars . . . . . . . . . 209

Division Organization Chart . . . . . . . . . . . . . . . . 239

Author Index . . . . . . . . . . . . . . . . . . . . . . . . 241 
Section 1

MATHEMATICAL SCIENCES 


\title{
1.0. INTRODUCTION
}

\author{
R. F. Sincovec
}

The Mathematical Sciences Section has three main objectives: to perform research in computer science, mathematics, and statistics germane to the solution of problems of national importance; to collaborate and consult with other scientists and engineers on such problems especially with respect to the computer science, mathematical and statistical aspects of the problems; and to maintain expertise in advanced and leading-edge computer science and mathematical sciences technologies with particular attention to technologies related to advanced large-scale parallel computing and heterogeneous distributed computing. The Mathematical Sciences Section actively pursues partnerships with industry and universities to leverage our research programs and to transfer our technology.

The research program in computer science spans three broad areas: the development of cumputational techniques for efficiently solving Grand Challenge problems, basic research in heterogeneous distributed computing, and the development of algorithms for efficiently perficming matrix computations on advanced computer architectures. Recent highlights of the algorithmic research include the development and analysis of new serial and parallel algorithms for the ordering, symbolic factorization, and numerical factorization of sparse matrix problems, the development and analysis of scalable algorithms for linear algebra problems, the development of a more efficient parallel implementation of conjugate gradient methods, and the development of fast techniques for generating optimal piecewise polynomial models to histogram data. Five Grand Challenge problems are being worked on in MSR in conjunction with the Center for Computational Sciences: groundwater transport, first principles materials calculations, global climate modeling, numerical Tokomak, and laser ablation. These are fundamental problems of particular interest to $\mathrm{DOE}$ and industry. We have continued to develop and maintain PICL, a portable instrumented communication library and ParaGraph, a graphical display system for visualizing the performance of these Grand Challenges. We have continued to evaluate new computers for their viability to solve important problems in science and industry. Recent highlights in heterogeneous distributed computing include developments in fault tolerance, load balancing, and multiprocessor integration with the best ideas being incorporated into PVM. PVM is a software package that allows the utilization of a heterogeneous network of parallel and serial computers as a single computational resource. It is quickly becoming a worldwide defacto standard for distributed computing. A debugging package fbr PVM programs called Xab was investigated. We have also begun development of a new tool on top of PVM called HeNCE that supports the creation, compilation, execution, debugging, and analysis of parallel programs for a heterogeneous group of computers.

Research in applied mathematics diring the reporting period emphasized the mathematical modeling of environmental problems, and their numerical solution on parallel supercomputers. Particular noteworthy accomplishments are (a) the completion of a cooling model for simulating waste site remediation by In-Situ Vitrification; (b) parallel implementation of a groundwater flow model and its application in a study of a particular waste site (WAG-6) at ORNL; (c) improved parallel algorithms for a MIMD implementation of the Global Community Climate Model; (d) development of optimal control techniques for application in "pump-and- 
treat" and bioremediation methods; and (e) the development of a computationally efficient Symmetric Galerkin method for boundary integral analysis. Important ORNL collaborations outside the mathematical sciences section have been with the Solid State Division (parallel molecular dynamics, visualization, thin film stability), Fusion Energy Division (numerical Tokamak), Environmental Sciences (groundwater modeling, In-Situ Vitrification, pore scale analysis), Engineering Physics and Mathematics (robotics), and the Chemical Technology Division (fluid drop motion).

In statistics, our basic research focus has continued to be in the area of computational. statistics and biostatistics. Some of the highlights include the development of an algorithm for use by computational scientists in the design and analysis of computer experiments and the use of derivatives in surface prediction for computer models, the development of methods for estimating cumulative radiation exposures using historical data that are subject to bias and uncertainty, the development of new methods of DNA sequence analysis based on probabilistic models, the development of an algorithm for data-driven global model search with respect to a model optimality criterion within a large user defined class of models, and the development of a methodology for analyzing large scientific datasets by extracting features indicative of changes in the process worthy of further investigation. Collaboration was initiated with the University of Maryland and the University of Tennessee on methods for the control of a nonlinear dynamical systems. Our applied, or collaborative, research has involved our statisticians with work in most of the ORNL divisions and major organizations in Martin Marietta Energy Systems. Examples that illustrate the diversity of this collaborative research are quality control methods for detecting outliers in large data sets, estimation of population size and survival probabilities of Canada geese using tag and recapture/resighting models, site characterization for waste cleanup using new interpolative techniques, and the development of Data Quality Objectives (DQO's) for projects associated with Environmental Restoration and Waste Management projects. Work has continued with the Y-12 Maintenance and Utilities Division on the development and establishment of a reliability centered maintenance (RCM) program. A growing source of consulting activities is defining sampling and methodological schemes for environmental restoration programs.

The computational resources of the Mathematical Sciences Section are concentrated in the Advanced Visualization Laboratory (AVL) and the Center for Computational Sciences (CCS) which together consist of a number of multiprocessor systems and the infrastructure required to support the use of these computers. The primary computational resource of the AVL is an Intel iPSC/860 with 128 processors, each with 8 MBytes of local memory, and capable of sustained performance in excess of 2 Gigaflops. This computer is currently being used on some of ORNL's Grand Challenge problems. A high speed link connects the AVL with the University of Tennessee (UT) Computer Science Department to facilitate the use of computers at both sites. UT computers include a CM-5 with 32 processors and a MasPar MP-2 with 4,096 processors. The primary CCS computers are two Intel Paragon computers, one with 512 processors and the other with 66 processors, and a Kendall Square KSR-2 with 64 processors. There are a number of other smaller MPP systems available plus an extensive infrastructure of Sun and IBM workstations on a local area networks with various compute, network, file, and print servers. 


\section{COMPUTER SCIENCE}

\section{General Parallel Computing}

1.1

\section{A USER'S GUIDE TO PVM PARALLEL VIRTUAL MACHINE}
A. Beguelin
J. J. Dongarra
G. A. Geist
R. Manchek*
V. Sunderam ${ }^{\dagger}$

(Abstract of ORNL/TM-11826, July 1991)

This report is the PVM Version 2.3 User's Guide. It contains an overview of PVM and how it is installed and used. Example programs in C and Fortran are included.

PVM stands for Parallel Virtual Machine. It is a software package that allows the utilization of a heterogeneous network of parallel and serial computers as a single computational resource. PVM consists of two parts: a daemon process that any user can install on a machine, and a user library that contains routines for initiating processes on other machines, for communicating between processes, and synchronizing processes.

Research sponsored by Applied Mathematical Sciences Research Program, U.S. DOE Office of Energy Research.

*University of Tennessee, Knoxville, TN.

†Emory University, Atlanta, GA.

\section{2}

\section{GRAPHICAL DEVELOPMENT TOOLS FOR NETWORK-BASED CONCURRENT SUPERCOMPUTING}
A. Beguelin
J. J. Dongarra
G. A. Geist
R. Manchek*
V. S. Sunderam ${ }^{\dagger}$

\begin{abstract}
[Abstract of paper presented at Supercomputing ' 91 Confernce, Albuquerque, NM, November 18-22, 1991; Proc. pp. $435-445$ (1991)]
\end{abstract}

This paper describes and X-window based software environment called HeNCE (Heterogeneous Network Computing Environment) designed to assist scientists in developing parallel programs that run on a network of computers. HeNCE is built on top of a software package called PVM which supports process management and communication between a network of heterogeneous computers. HeNCE is based on a parallel programming paradigm where an application program can be described by a graph. Nodes of the graph represent subroutines and the arcs represent data dependencies. HeNCE is composed of integrated graphical tools for creating, compiling, executing, and analyzing HeNCE programs.

Research sponsored by Applied Mathematical Sciences Research Program, U.S. DOE Office of Energy Research.

*University of Tennessee, Knoxville, TN.

$\dagger^{\prime}$ Emory University, Atlanta, GA.

\section{3}

\section{HeNCE: GRAPHICAL DEVELOPMENT TOOLS FOR NETWORK-BASED CONCURRENT COMPUTING}
A. Beguelin
J. J. Dongarra
G. A. Geist
R. Manchek*
K. Moore*
R. Wade*
V. Sunderam ${ }^{\dagger}$

[Abstract of paper presented at the Scalable High Performance Computing Conference, Williamsburg, VA, April 26-29, 1992; Proc. J. Saltz and R. Voigt, Eds., IEEE Computer Society Press (1992)]

HeNCE (Heterogeneous Network Computing Environment) is an $\mathrm{X}$ Window based graphical parallel programming environment that was created to assist scientists and engineers with the development of parallei programs. HeNCE provides a graphical interface for creating, compiling, executing, and debugging parallel programs, as well as configuring a distributed virtual computer (using PVM). HeNCE programs can be run on a single Unix workstation or over a network of heterogeneous machines. This paper describes the purpose and use of the HeNCE software.

\footnotetext{
Research sponsored by Applied Mathematical Sciences Research Program, U.S. DOE Office of Energy Research and the National Science Foundation's Science and Technology Center Cooperative.

*University of Tennessee, Knoxville, TN.

$\dagger^{\dagger}$ Emory University, Atlanta, GA.
} 
1.4

\section{HETEROGENEOUS NETWORK COMPUTING}
A. Beguelin
J. J. Dongarra
G. A. Geist
R. Manchek*
V. Sunderam ${ }^{\dagger}$

[Abstract of Supercomputer Review $\$(9), 44$ (1991)]

Heterogeneous networks of computers are becoming commonplace in high-performance computing. Systems ranging from workstations to supercomputers are linked together by high speed networks. Until recently each computing resource on the network remained a separate unit, but now 20-30 institutions worldwide are writing and running truely heterogeneous programs utilizing multiple computer systems to solve applications through the use of a software package called PVM.

PVM stands for Parallel Virtual Machine. PVM is designed from the ground up with heterogeneity and portability as primary goals. As such it is one of the first software systems that allows machines with wildly different architectures and floating point representations to work together on a single computational task.

The Heterogeneous Network Project, being worked on by researchers at Oak Ridge National Laboratory, the University of Tennessee, and Emory University, is involved in the research and development of two software packages specifically designed to facilitate heterogeneous parallel computing. The first package is PVM, which can be used on its own or as a foundation upon which other heterogeneous network software can be built. The second package, still in the early stages of development, is called HeNCE, which stands for Heterogeneous Network Computing Environment. HeNCE is being built on top of PVM with the intention of simplifying the task of writing, compiling, running, debugging, and analyzing programs on a heterogeneous network. The goal is to make network computing accessable to scientists and engineers without the need for extensive training in parallel computing and allowing them to use resources best suited for a particular phase of the computation.

What follows is a description of the basic features of these two packages, and descriptions of our experiences with using PVM.

Research sponsored by Applied Mathematical Sciences Research Program, U.S DOE Office of Energy Research.

* University of Colorado, Boulder, CO.

${ }^{\dagger}$ Emory University, Atlanta, GA.
1.5

\section{SPEEDUP PROPERTIES OF \\ PHASES IN THE EXECUTION \\ PROFILE OF DISTRIBUTED \\ PARALLEL PROGRAMS}

\author{
B. M. Carlson* \\ L. W. Dowdy ${ }^{\dagger}$ \\ T. D. Wagner ${ }^{\dagger}$ \\ P. H. Worley
}

(Abstract of paper presented at the 6th International Conference on Modeling Techniques and Tools for Computer Performance Evaliation, Edinburgh, Scotland, September 16-18, 1992; Proc. pp. 83-95 (1992); also ORNL/TM11900, August 1992]

The execution profile of a distributed-memory parallel program specifies the number of busy processors as a function of time. Periods of homogeneous processor utilization are manifested in many execution profiles. These periods can usually be correlated with the algorithms implemented in the underlying parallel code. Three families of methods for smoothing execution profile data are presented. These approaches simplify the problem of detecting end points of periods of homogeneous utilization. These periods, called phases, are then examined in isolation, and their speedup characteristics are explored. A specific workload executed on an Intel iPSC/860 is used for validation of the techniques described.

Research sponsored by Applied Mathematical Sciences Research Program, U.S. DOE Office of Energy Research.

* University of Toronto, Ontario, Canada.

$\dagger^{\dagger}$ anderbilt University, Nashville, TN.

\section{6}

\section{COMMUNICATION PERFORMANCE OF THE INTEL TOUCHSTONE DELTA MESH}

\section{T. H. Dunigan}

(Abstract of ORNL/TM-11983, January 1992)

The communication performance of the i860based Intel DELTA mesh supercomputer is compared with the Intel iPSC/860 hypercube and the Ncube 6400 hypercube. Single and multiple hop communication bandwidth and latencies are measured. Concurrent communication speeds and speed under network load are also measured. File I/O performance of the mesh-attached Concurrent File System is measured.

Research sponsored by Applied Mathematical Sciences Research Program, U.S. DOE Office of Energy Research. 
1.7

\section{HYPERCUBE CLOCK SYNCHRONIZATION}

\section{T. H. Dunigan}

[Abstrace of Concurrency: Practice \& Experience 4(3), 257 (1992)]

Algorithms for synchion:zing the times and frequencies of the clocks of Intel and Ncube hypercube multiprocessors are presented. Bounds for the error in estimating clock offsets and frequencies are formulated in terms of the clock read error and message transmission time. Clock and communication performance of the Ncube and Intel hypercubes are analyzed, and performance of the synchronization algorithms is presented.

Research sponsored by Applied Mathematical Sciences Research Program, U.S. DOE Office of Energy Research.

1.8

\section{KENDALL SQUARE MULTIPROCESSOR: EARLY EXPERIENCES AND PERFORMANCE}

\section{T. H. Dunigan}

(Abstract of ORNL/TM-12065, April 1992)

Initial performance results and early experiences are reported for the Kendall Square Researcis Multiprocessor. The basic architecture of the shared-memory multiprocessor is described, and computational and $1 /()$ performance is measured for both serial and $r$ arallel programs. Experiences in porting various applications are described.

Research sponsored by Applied Mathematical Sciences Research Program, U.S. DOE Office of Energy Research.

\section{9}

\section{PERFORMANCE OF TKE INTEL IPSC/860 AIND NCUBE 6400 HYPERCUBES}

\section{T. H. Dunigan}

[Abstract of Perallel Comprting 27, 1285 (1991); also ORNL/TM-1!790, April 1991]

The performance of the Intel $\mathrm{iPSC} / 860 \mathrm{~b} /-$ percube and the Ncube 6400 hy. ercube are com- pared with earlier hypercubes from Intel and Nicube. Computation and communication performance for a number of low-level benchmarks are presented for the Intel iPSC $/ 1, \mathrm{iPSC} / 2$, and iPSC/860 and for the Ncube 3200 and 6400 . File I/O performance of the iPSC/860 and Ncube 6400 are compared.

Research sponsored by Applied Mathematical Sciences Research Program, U.S. DOE Offic : of Energy Research.

\subsection{0}

\section{PARALLEL SUPERCONDUCTOR CODE EXCEEDS 2.5 Gflops ON iPSC $/ 860$}
G. \&. Geist
B. Ginatempo *
W. A. Shelton ${ }^{\dagger}$
G. M. Stocks ${ }^{\ddagger}$

(Abstract of Journal of Supercomputing (in press); also paper presented at the Supercomputing ' 91 Conference, Albuquerque, NM, November 18-22, 1991; Proc. (1991)]

Researchers at Oak Ridge National Laboratory have develored a Fortran application code for calculating tie electronic properties and energetics of disordered materials. The same source code has been compiled and run on workstations, Crays, and the Intel iPSC/860. This electronic structures code is capable of running over 2 Gflops on both an 8 processor Cray YMP and a 128 processor Intel iPSC $/ 860$.

Using this new KKR-CPA code, densityof-state computations of a perovskite superconductor executed at a rate of 2527 Mflops on the Intel iPSC/860. This corresponds to a price/performance rate of 842 Mflops per \$1 million based on the list price of this computer. Similar, but smaller, computations done on a network of $10 \mathrm{IBM} R S / 6000$ workstations and also a single IBM RS/ 6000 model 320 executed at a price/performance rate of 1.29 Gflops per $\$ 1$ million and 2.23 Gflops per $\$ 1$ million respectively.

Research sponsored by Applied Mathematical Sciences Research Program, U.S. DOE Office of Energy Research.

* Universita of Messina, Italy.

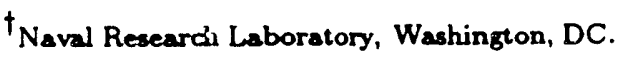

† Metals and Ceramics Division. 
1.11

\section{SOLVING MATERIAL SCIENCE PROBLEMS AT ORNL'S CENTER FOR COMPUTATIONAL STUDIES}

\author{
G. A. Geist
}

(. . ostract of paper presented at the SIAM 40th Anniversary Meeting, Los Angeles, CA, July 20-24, 1992)

Several computational Grand Challenge problems are being addressed at Oak Ridge $\mathrm{Na-}$ tional Laboratory. This talk will address one of these problems - the calculation of the electronic structure of solids from first principles. The talk will describe the problem and an algorithm for the solution of substitutionally disordered materials. We will point out where parallelization and other performance enhancements have been employed.

We will compare our experiences with running this application on a 8 processor Cray YMP, a 128 processor Intel iPSC $/ 860$, a network of Cray processors, and a 512 processor Intel Paragon.

Research sponsored by Applied Mathematical Sciences Research Program, U.S. DOE Office of Energy Research.

\subsection{2}

THE PVM SYSTEM: SUPERCONIPUTER LEVEL CONCURRENT COMPUTATION ON A HETEROGENEOUS NETWORK OF WORKSTATIONS

\section{G. A. Geist V. S. Sunderam*}

[Abstract of paper presented at the 6th Distributed Memory Computing Conference, Portland, OR, April 28-May 2, 1991; Proc. pp. 258-261 (1991)]

The PVM (Parallel Virtual Machine) system enables supercomputer level concurrent computations to be performed on interconnected networks of heterogeneous computer systems. Specifically, a network of 13 IBM RS/6000 powerstations has been used to run superconductor modeling codes at more than 250 Mflops. This paper describes the PVM system and two example applications running $c n$ it.

Research sponsored by Applied Mathematical Sciences Research Frogram, U.S. DOE Office of Energy Research.

*Emory University, Atlanta, GA.
1.13

\section{NETWORK-BASED CONCURRENT COMPUTING ON THE PVM SYSTEM}

\begin{abstract}
G. A. Geist V. S. Sunderam*
[Abstract of Concurtency: Practice and Experience 4(4), 293 (1992); also ORNL/TM-11760, June 1991]

Concurrent computing environments based on loosely coupled networks have proven effective as resources for multiprocessing. Experiences with and enhancements to version 1.0 of PVM (Parallel Virtual Machine) are described in this paper. PVM is a software package that allows the utilization of a heterogeneous network of parallel and serial computers as a single computational resource. This report also describes an interactive graphical interface to PVM, and porting and performance results from production applications.
\end{abstract}

Research sponsored by Applied Mathematical Sciences Research Program, U.S. DOE Office of Energy Research.

*Emory University, Atlanta, GA.

\subsection{4}

\section{VISUALIZING THE PERFORMANCE - T PARALLEL PROGRAMS}

\section{T. Heath* J. A. Etheridge}

[Abstract of IEEE Software, p. 29 (Se-tember 1991); also ORNL/TM-11813, Ma* “9y1]

Graphical visualization aids human comprehension of complex phenomena and large volumes of data. The behavior of parallel programs on advanced architectures is often extremely complex, and monitoring the performance of such programs can generate vast quantities of data. So it seems natural to use visualization to gain insight into the behavior of parallel programs so we can better understand them and improve their performance.

We have developed ParaGraph, a software tool that provides a detailed, dynamic, graphical animation $u$ the behavior of message-passing parallel programs and graphical summaries of their performance.

Research sponsored by Applied Mathematical Sciences Research Program, U.S. DOE Office of Energy Research.

* University of Ilinois, Urbana, IL. 
1.15

\section{PARALLEL COMPUTERS TAKE LEAD IN COMPLEX PROBLEM SOLVING}

\author{
M. R. Leuze R. C. Ward
}

[Abstract of Computers in Physics 5(6), 582 (1991)]

Parallel computing concepts are not new; many early computers used parallelism in some form. However, it is only within the last 20 years that true multiprocessor systems have been constructed, and only within the last 6 to 8 years that trese systems have become cost effective. Today, the most powerful computers in existence are parallel. Despite many remaining problems, numerous applications programs at the leading edge of scientific research are exploiting the power of these machines.

Research sponsored by Applied Mathematical Sciences Research Program, U.S. DOE Office of Energy Research.

\subsection{6}

\section{DEVELOPING LARGE SCALE APPLICATIONS BY INTEGRATING PVM AND THE INTEL IPSC $/ 860$}

\author{
W. A. Shelton \\ G. A. Geist
}

(Abstract of paper presented at the 1992 Annual Intel Supercomputer Users' Group Conference, Dalles, TX, October 4-7, 1992)

We have developed a hybrid parallel computer code for calculating the electronic structure of both ordered and substutionally disordered alloys. This computer algorithm uses PVM (Parallel Virtual Machine) to pass messages to a node on the Intel iPSC/860 and in turn this node broadcasts these messages, using Intel iPSC/860 commands, to the other nodes in the user's application. By using PVM we can integrate multiple Intel machines into our computing environment while avoiding the problem of insufficient memory due to a large host code. This enables us to calculate the electronic structure of large scale systems that would otherwise be unattainable due to a lack of resources. For example, we present the first self-consistent scalar-relativistic electronic structure calculations on the substutionally disordered high temperature superconducting compound $\mathrm{Ba}_{1-x} \mathrm{~K}_{x} \mathrm{BiO}_{3}$.

Research sponsored by U.S. Department of Energy.

\subsection{7}

\section{FERMI SURFACES OF $\beta$-PHASE NiAl ALLOYS}

\author{
G. M. Stocks* W. A. Shelton \\ D. M. Nicholson* G. A. Geist \\ F. J. Pinski ${ }^{\dagger}$
}

(Abstract of paper presented at the Southeastern Section Meeting of the American Physical Society, Oak, Ridge, TN, November 12-14, 1992; Proc. Vol. 37(7), p. 1646 (1992)]

At the stoiciometric composition ( $c=0.5) \mathrm{B}$ phase $\mathrm{Ni}_{c} \mathrm{Al}_{(1-c)}$ alloys form in the $\mathrm{CsCl}$ structure, for $0.5<c<0.625$ the excess $\mathrm{Ni}$ atoms substitute on the Al sublattice as antisite defects. We have calculated the effects of substitutional disorder electronic structure of these alloys using the complex lattice KKR-CPA method. We show the evolution of the Fermi surface with composition. The disorder caused by the random distribution of $\mathrm{Ni}$-antisite defects results in complex $\mathrm{k}$-dependent smearing of the alloy Fermi surface. For $c=0.625$ the Fermi surface is sharp over those parts identified by Zhao and Harmon as giving rise to phonon softening. Here the evolution of this spanning vector is followed over the entire composition range. We advocate the use of ACAR positron annihilation and angle resolved photo-emission experiments to measure the details of the topology of the Fermi surface in the KKR-CPA Bloch spectral functions.

Research sponsored by Division of Materials Science, Office of Basic Energy Sciences; by Assistant Secretary for Conservation and Renewable Energy, Office of Industrial Technologies; by AIC Materials Program; and by Applied Mathematical Sciences Research Program, Office of Energy Research, U.S. DOE.

* Metals and Ceramics Division.

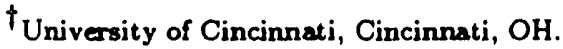


1.18

\section{THE PVM SYSTEM: SUPERCOMPUTER LEVEL CONCURRENT COMPUTATION ON A NETWORK OF IBM RS/6000 POWERSTATIONS}

\section{$\begin{array}{ll}\text { V. S. Sunderam* } & \text { G. A. Geist }\end{array}$}

(Abstract of paper presented at the IBM Large Scale Computer Analysis and Modeling Conference, Yorktown, NY, April 24-26, 1991)

The PVM (Parallel Virtual Machine) system enables supercomputer level concurrent computations to be performed on interconnected networks of heterogeneous computer systems. Specifically, a network of 13 IBM RS/6000 powerstations has been successfully used to execute production quality runs of superconductor modeling codes at more than 250 Mflops. This work demonstrates the effectiveness of cooperative concurrent processing for high performance applications, and shows that supercomputer level computations may be attained at a fraction of the cost on distributed computing platforms.

This paper describes the PVM programming environment and user facilities, as they apply to hardware platforms comprising a network of IBM RS/6000 powerstations. The salient design features of PVM will be discussed; including heterogeneity, scalability, multilanguage support, provisions for fault tolerance, the use of multiprocessors and scalar machines, an interactive graphical front end, and support for profiling, tracing, and visual analysis.

The PVM system has been used extensively, and a range of production quality concurrent applications have been successfully executed using PVM on a variety of networked platforms. The paper will mention representative examples, and discuss two in detail. The first is a material sciences problem that was originally developed on a Cray 2. This application code calculates the electronic structure of metallic alloys from first principles and is based on the KKR-CPA algorithm. The second is a molecular dynamics simulation for calculating materials properties. Performance results for both applications on networks of RS/6000 powerstations will be presented, and accompanied by discussions of the other advantages of PVM and its potential as a complement of alternative to conventional supercomputers.

Research sponsored by Applied Mathematical Sciences Research Program, U.S. DOE Office of Energy Research.

*Emory University, Atlanta, GA.

1.19

\section{STANDARDS FOR MESSAGE- PASSING IN A DISTRIBUTED MEMORY ENVIRONMENT}

\author{
D. W. Walker
}

(Abstract of ORNL/TM-12147, August 1992)

This report presents a summary of the main ideas presented at the First CRPC Workshop on Standards for Message Passing in a Distributed Memory Environment, held April 29-30, 1992, in Williamsburg, Virginia. This workshop attracted 68 attendees including representatives from major hardware and software vendors, and was the first in a series of workshops sponsored by tize Center for Research on Parallel Computation. The aim of this series of workshops is to develop and implement a standard for message passing; on distributed memory concurrent computers, thereby making it easier to develop efficient, portable application codes for such machines. The report discusses the main issues raised in the CRPC workshop, and describes proposed desirable features of a message passing standard for distributed memory environments.

Research sponsored by Applied Mathematical Sciences Research Program, U.S. DOE Office of Energy Research.

\subsection{0}

\section{A NEW PICL TRACE FILE FORMAT}

$$
\text { P. H. Worley }
$$

(Abstract of ORNL/TM-12125, October 1992)

A trace file format is described that will be used in future releases of the Portable Instrumented Communication Library (PICL) and ParaGraph. The new format provides improved sup- 
port for tracing and profiling PICL communication primitives and user-defined events. The new format is also easily extended and may be useful in other instrumentation packages and performance visualization tools.

Research sponsored by Applied Mathematical Sciences Research Program, U.S. DOE Office of Energy Research.

\subsection{1}

\section{PHASE MODELING OF A PARALLEL SCIENTIFIC CODE}

\section{P. H. Worley}

(A) stract of paper presented at the Scalable High Performance Computing Conference, Williamsburg, VA, April 26-29, 1992; Proc. pp. 322-327, J. Saltz and R. Voigt, Eds., IEEE Computer Society Press (1992)]

This paper describes a performance model for a parallel program that solves the nonlinear shallorv water equations using the spectral transform method. The model is generated via a phase analysis, and consists of a sequence of simple models whose sum describes the performance of the entire code. This use of a sequence of simple models increases the range of validity of the model as the problem and machines parameters scale.

Research sponsored by Applied Mathematical Sciences Research Program, U.S. DOE Office of Energy Research.

1.22

\section{PICL: PAST, PRESENT, AND FUTURE}

\section{P. H. Worley}

(Abstract of paper presented at the 1992 Annual Intel Supercomputer Users' Group Conference, Dallas, TX, October 4-7, 1992)

We describe the motivation and history behind the development of PICL, the portable instrumented communication library. We discuss the role PICL plays in research in performance modeling and in the development of Grand Challenge application codes at Oak Ridge National Laboratory (ORNL). We also describe some of the uses of PICL by researchers outside of ORNL. We describe the current status of PICL, including the new trace file format and the new tracing com- mands. We then describe planned extensions to PICL, and PICL's relationship to PVM and the efforts on standardizing message-passing interfaces.

Research sponsored by Applied Mathematical Sciences Research Program, U.S. DOE Office of Energy Research.

1.23

\section{THE EFFECT OF MULTIPROCESSOR RADIUS ON SCALING}

\author{
P. H. Worley \\ [Abstract of Parallel Computing 18(1), 361 (1992)
}

In earlier work, it was established that, for a large class of linear partial differential equations (PDEs), increasing the problem size necessarily increases the amount of data that is needed to approximate the solution of some scalar value in the solution field. In consequence, increasing the problem size of these PDEs increases the execution time, independent of the algorithm, the number of processors, and the interprocessor communication network used to solve the problem. In this paper, the analysis is extended to take into account the effect of the radius of the interprocessor communication network on the growth in the execution time. It is shown that the increasing amount of data also contributes to an increase in the time spent in interprocessor communication in optimal parallel algorithms for the problem. In particular, for any physically-realizable multiprocessor, the time spent on interprocessor communication will be the dominant constraint on the performance of optimal algorithms when the problem and multiprocessor sizes are large.

Research sponsored by Applied Mathematical Sciences Research Program, U.S. DOE Office of Energy Research. 


\section{Dense Matrix Computations}

\subsection{4}

\section{THE DESIGN OF SCALABLE SOFTWARE LIBRARIES FOR DISTRIBUTED MEMORY CONCURRENT COMPUTERS}

\author{
J. Choi J. J. Dongarra \\ D. W. Walker
}

(Abstract of paper presented at the CNRS-NSF Collaborative Workshop on Environments and Tools for Parallel Scientific Computing, St. Hilaire du Touvet, France, September 7-8, 1992)

This paper describes the design of ScalAPACK, a scalable software library for performing dense and banded linear algebra computations on distributed memory concurrent computers. The specification of the data distribution has important consequences for interprocessor communication and load balance, and hence is a major factor in determining performance and scalability of the library routines. The block scattered data distribution is adopted as a simple, yet general-purpose, way of decomposing block-partitioned matrices. Distributed memory versions of the Level 3 BLAS provide an easy and convenient way of implementing the ScaLAPACK routines. Performances and scalability results for the ScaLAPACK LU factorization routine are presented, and potential uses for ScaLAPACK are discussed.

Research sponsored by Applied Mathematical Sciences Research Program, U.S. DOE Office of Energy Research and by DARPA and ARO.

\subsection{5}

\section{LEVEL 3 BLAS FOR DISTRIBUTED MEMORY CONCURRENT COMPUTERS}

\section{J. Choi J. J. Dongarra} D. W. Walker

(Abstract of paper presented at the CNRS-NSF Collaborative Workshop on Environments and Tools for Parallel Scientific Computing, St. Hilaire du Touvet, France, September 7-8, 1992)

This paper discusses issues in developing a version of the Level 3 BLAS distributed memory concurrent computers. The Level 3 BLAS are particularly important whrn using machines with hierarchical memory as they maximize reuse of data in the upper levels of the memory hierarchy. In implementing the distributed Level 3 BLAS a block scattered decomposition is employed. Details of the parallel implementation of DGEMM, for performing matrix-matrix multiplication, and DTRSM, for solving triangular systems, are given, and results are presented for runs on the Intel Touchstone Delta computer.

Research sponsored by Applied Mathematical Sciences Research Program, U.S. DOE Office of Energy Research and by DARPA and ARO.

\subsection{6}

SCaLAPACK: A SCALABLE
LINEAR ALGEBRA LIBRARY
FOR DISTRIBUTED MEMORY
CONCURRENT COMPUTERS

$$
\begin{gathered}
\text { J. Choi J. Dongarra } \\
\text { D. W. Walker R. Pozo* }
\end{gathered}
$$

(Abstract of paper presented at the 4th Symposium on Frontiers of Massively Parallel Computing, McLean, VA, October 19-21, 1992; Proc. pp. 120-127 (1992)]

This paper describes ScalAPACK, a distributed memory version of the LAPACK software package for dense and banded matrix computations. Key design features are the use of distributed versions of the Level 3 BLAS as building blocks, and an object-based interface to the library routines. The square block scattered decomposition is described. The implementation of a distributed memory version of the right-looking LU factorization algorithm on the Intel Delta multicomputer is discussed, and performance results are presented that demonstrate the scalability of the algorithm.

Research sponsored by Applied Mathematical Sciences Research Program, U.S. DOE Office of Energy Research.

*University of Tennessee, Knoxville,TN. 
1.27

\section{NUMERICAL CONSIDERATIONS IN COMPUTING INVARIANT SUBSPACES}

\author{
J. J. Dongarra \\ S. Hammarling* \\ J. H. Wilkinson ${ }^{\dagger}$ \\ [Abstract of SIAM J. Matrix Anal. Appl. 13(1), 145
} (1992)]

This paper describes two methods for computing the invariant subspace of a matrix. The first method involves using transformations to interchange the eigenvalues. The matrix is as sumed to be in Schur form and transformations are applied to interchange neighboring blocks. The blocks can be either one by one or two by two. The second method involves the construction of an invariant subspace by a direct computation of the vectors, rather than by applying transformations to move the desired eigenvalues to the top of the matrix.

Research sponsored by Applied Mathematical Sciences Research Program, U.S. DOE Office of Energy Research; by the National Science Foundation; and by the Science Alliance Program at the University of Tennessee.

*Numerical Algorithms Group Ltd., Oxford, United Kingdom.

$\dagger_{\text {Deceased. }}$

1.28

\section{THE IBM RISC SYSTEM/6000 AND LINEAR ALGEBRA OPERATIONS}

\section{J. J. Dongarra P. Mays* G. R. di Brozolo ${ }^{\dagger}$}

[Abstract of Supercomputer (in press)]

This paper discusses the IBM RISC System $/ 6000$ workstation and a set of experiments with blocked algorithms commonly used in solving problems in numerica! linear algebra. We describe the performance o: these algorithms and discuss the techniques used in achieving high performance on such an architecture.

Research sponsored by Applied Mathematical Sciences Research Program, U.S. DOE Office of Energy Research and by the National Science Foundation and Technology Center.

*Numerical Algorithms Group Ltd., Oxford, United Kingdom.

${ }^{\dagger}$ IBM European Center for Science and Engineering Computation, Giorgione, Italy.

\subsection{9}

\section{NA-NET NUMERICAL ANALYSIS NET}

\section{J. J. Dongarra B. Rosener*}

(Abstract of ORNL/TM-11986, December 1991)

This report describes a facility called NANET created to allow numerical analysts (na) an easy method of communicating with one another. The main advantage of the NA-NET is uniformity of addressing. All mail is addressed to the Internet host "na-net.ornl.gov" at Oak Ridge National Laboratory. Hence, members of the NA-NET do not need to remember complicated addresses or even where a member is currently located. As long as moving members change their e-mail address in the NA-NET everything works smoothly.

The NA-NET system is currently located at Oak Ridge National Laboratory. It is running on the same machine that serves netlib. Netlib is a separate facility that distributes mathematical software via electronic mail. For more information on netlib consult, or send the one-line message "send index" to netlib@ornl.gov. The following report describes the current NA-NET system from both a user's perspective and from an implementation perspective. Currently, there are over 2100 members in the NA-NET. An average of 110 mail messages pass through this facility daily.

Research sponsored by Applied Mathematical Sciences Research Program, U.S. DOE Office of Energy Research and by the National Science Foundation.

*University of Tennessee, Knoxville, TN. 


\section{A PARALLEL ALGORITHM FOR THE NON-SYMMETRIC EIGENVALUE PROBLEM}

\author{
J. J. Dongarra M. Sidani*
}

(Abstract of ORNL/TM-12003, December 1991)

This paper describes a parallel algorithm for computing the eigenvalues and eigenvectors of a non-symmetric matrix. The algorithm is based on a divide-and-conquer procedure and uses an iterative refinement technique.

Research sponsored by Applied Mathematical Sciences Research Program, U.S. DOE Office of Energy Research and by the National Science Foundation.

*University of Tennessee, Knoxville, TN.

\subsection{1}

\section{REDUCTION TO CONDENSED FORM FOR THE EIGENVALUE PROBLEM ON DISTRIBUTED MEMORY ARCHITECTURES}

\section{J. J. Dongarra R. A. van de Geijn*}

(Abstract of ORNL/TM-12006, January 1992)

In this paper, we describe a parallel implementation for the reduction of general and symmetric matrices to Hessenberg and tridiagonal form, respectively. The methods are based on LAPACK sequential codes and use a panel-wrapped mapping of matrices to nodes. Results from experiments on the Intel Touchstone Delta are given.

Research sponsored by Applied Mathematical Sciences Research Program, U.S. DOE Office of Energy Research and by the National Science Foundation and Technology Center.

* University of Texas, Austin, TX.

\section{A LOOK AT SCALABLE DENSE LINEAR ALGEBRA LIBRA RIES}

\author{
J. J. Dongarra R. A. van de Geijn* \\ D. W. Walker
}

[Abstract of ORNL/TM-12126, July 1992; also paper presented at the Scalable High Performance Computing Conference, Williamsburg, VA, April 26-29, 1992; Proc. J. Saltz and R. Voigt. Eds., IEEE Computer Society Press (1992)]

We discuss the essential design features of a library of scalable software for performing dense linear algebra computations on distributed memory concurrent computers. The square block scattered decomposition is proposed as a flexible and general-purpose way of decomposing most, if not all, dense matrix problems. An object-oriented interface to the library permits more portable applications to be written, and is easy to learn and use, since details of the parallel implementation are hidden from the user. Experiments on the Intel Touchstone Delta system with a prototype code that uses the square blocis scattered decomposition to perform $\mathrm{LU}$ factorization are presented and analyzed. It was found th:at the code was both scalable and efficient, performing at about $14 \mathrm{Gflop} / \mathrm{s}$ (double precision) for the largest problem considered.

Research sponsored by Applied Mathematical Sciences Research Program, U.S. DOE Office of Energy Research and by the Defense Advanced Research Projects Agency.

*University of Texas, Austin, TX.

\subsection{3}

\section{A DIVIDE AND CONQUER APPROACH TO THE NONSYMMETRIC EIGENVALUE PROBLEM}

\section{E. R. Jessup}

(Abstract of paper presented at the 13th IMACS World Congress on Computation and Applied Mathematics Dublin, Ireland, July 21-26, 1991; Proc. Vol. 1 (1991 î]

Serial computation combined with high communication costs on distributed-memory multiprocessors make parallel implementations of the QR method for the nonsymmetric eigenvalue problem 
inefficient. This paper introduces an alternative algorithm for the nonsymmetric tridiagonal eigenvalue problem based on rank two tearing and updating of the matrix. The parallelism of this divide and conquer approach stems from independent solution of the updating problems.

Research sponsored by Applied Mathematical Sciences Research Program, U.S. DOE Office of Energy Research.

\subsection{4}

\section{A CASE AGAINST A DIVIDE CONQUER APPROACH TO THE NONSYMMETRIC EIGENVALUE PROBLEM}

\section{E. R. Jessup}

(Abstract of ORNL/TM-11903, December 1991)

Divide and conquer techniques based on rankone updating have proven fast, accurate, and efficient in parallel for the real symmetric tridiagonal and unitary eigenvalue problems and for the bidiagonal singular value problem. Although the divide and conquer mechanism can also be adapted to the real nonsymmetric eigenproblem in a straightforward way, most of the desirable characteristics of the other algorithms are lost. In this paper, we examine the problems of accuracy and efficiency that can stand in the way of a nonsymmetric divide and conquer eigensolver based on low-rank updating.

Research sponsored by Applied Mathematical Sciences Research Program, U.S. DOE Office of Energy Research.
1.35

\section{EXPERIMENTS WITH AN ORDINARY DIFFERENTIAL EQUATION SOLVER IN THE PARALLEL SOLUTION OF METHOD OF LINES PROBLEMS ON A SHARED MEMORY PARALLEL COMPUTER, PART II}

\author{
D. K. Kahaner* \\ W. E. Schiesser ${ }^{\dagger}$ \\ E. G. $\mathrm{Ng}$ \\ S. Thompson
}

(Abstract of paper presented at the 7th International Association for Mathematics and Computer Simulation Conference on Computer Methods for Partial Differential Equations, New Brunswick, NY, June 21-24, 1992; Proc. pp. 404-410 (1992)]

The authors previously considered the use of parallel direct dense matrix techniques for the solution of method of lines problems on a shared memory parallel computer. It was found that substantial speedups can be obtained for dense problems involving spatially discretized systems containing from a few to several hundred ordinary differential equations. This paper reports the results for similar experiments with parallel direct sparse matrix techniques for the solution of such problems. The methods are implemented in the context of a well-known stiff ordinary differential equation solver. Parallelism is exploited by forming the Jacobian matrix in parallel and by using parallel direct sparse linear algebra matrix techniques for the solution of the corrector equations. Timing results are given for several representative problems involving spatially discretized systems containing from several hundred to a few thousand ordinary differential equations. The experiments reported in this paper were performed on a Sequent Balance computer which is a parallel computer with 8 processors sharing a global memory. The results demonstrate that substantial speedups can be obtained in this manner for sparse method of lines problems.

Research sponsored by Applied Mathematical Sciences Research Program, U.S. DOE Office of Energy Research and by the Universities Research Association, Inc.

*National Institute of Standards, Gaithersburg, MD.

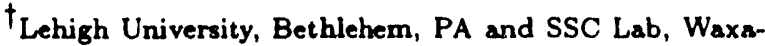
hachie, TX.

$\ddagger_{\text {Radford University, Radford, VA. }}$ 
1.36

\section{PARALLELIZING ACROSS TIME WHEN SOLVING TIME-DEPENDENT PARTIAL DIFFERENTIAL EQUATIONS}

\author{
P. H. Worley
}

(Abstract of ORNL/TM-11963, September 1991)

The standard numerical algorithms for solving time-dependent partial differential equations (PDEs) are inherently sequential in the time direction. This paper describes algorithms for the time-accurate solution of certain classes of linear hyperbolic and parabolic PDEs that can be par- allelized in both time and space and have serial complexities that are proportional to the serial complexities of the best known algorithms. The algorithms for parabolic PDEs are variants of the waveform relaxation multigrid method (WFMG) of Lubich and Ostermann where the scalar ordinary differential equations (ODEs) that make up the kernel of WFMG are solved using a cyclic reduction type algorithm. The algorithms for hyperbolic PDEs use the cyclic reduction algorithm to solve ODEs along characteristics.

Research sponsored by Applied Mathematical Sciences Research Program, U.S. DOE Office of Energy Research.

\section{Sparse Matrix Computations}

1.37

\section{AN INTRODUCTION TO CHORDAL GRAPHS AND CLIQUE TREES}

\section{J. R. S. Blair* $\quad$ B. W. Peyton}

(Abstract of ORNL/TM-12203, November 1992; also paper presented at the Institute for Mathematic and Its Applications Workshop on Sparse Matrix Computations: Graph Theory, Issues and Algorithms, Minneapolis, MN, October 14-18, 1991)

Clique trees and chordal graphs have carved out a niche for themselves in recent work on sparse matrix algorithms, due primarily to research questions associated with advanced computer architectures. This paper is a unified and elementary introduction to the standard characterizations of chordal graphs and clique trees. The pace is leisurely, as detailed proofs of all results are included. We also briefly discuss applications of chordal graphs and clique trees in sparse matrix computations.

Research sponsored by Applied Mathematical Sciences Research Program, U.S. DOE Office of Energy Research.

*University of Tennessee, Knoxville, TN.

\subsection{8 \\ ON FINDING MINIMUM-DIAMETER CLIQUE TREES}

\author{
$\begin{array}{ll}\text { J. R. S. Blair* } & \text { B. W. Peyton }\end{array}$
}

[Abstract of paper presented at the Computer Science Colloquim, Clemson University, Clemson, SC, October 7, 1992; also ORNL/TM-11850, August 1991; also SIAM J. on Discrete Mathematics (in press)]

It is well-known that any chordal graph can be represented as a clique tree (acyclic hypergraph, join tree). Since some chordal graphs have many distinct clique tree representations, it is interesting to consider which one is most desirable under various circumstances. A clique tree of minimum diameter (or height) is sometimes a natural candidate when choosing clique trees to be processed in a parallel computing environment.

This paper introduces a linear time algorithm for computing a minimum-diameter clique tree. The new algorithm is an analogue of the natural greedy algorithm for rooting an ordinary tree in order to minimize its height. It has potential application in the development of parallel algorithms for both knowledge-based systems and the solution of sparse linear systems of equations.

Research sponsored by Applied Mathematical Sciences Research Program, U.S. DOE Office of Energy Research.

*University of Tennessee, Knoxville, TN. 
1.39

\section{PREDICTING STRUCTURE IN NONSYMMETRIC SPARSE MATRIX FACTORIZATIONS}

\section{J. R. Gilbert* $\quad$ E. G. $\mathbf{N g}$}

(Abstract of ORNL/TM-12204, October 1992; also Institute for Mathematics and Its Applications Workshop on Sparse Matrix Computations: Graph Theory, Issues and Algorithms, Minneapolis, MN, October 14-18, 1991)

Many computations on sparse matrices have a phase that predicts the nonzero structure of the output, followed by a phase that actually performs the numerical computation. We study structure prediction for computations that involve nonsymmetric row and column permutations and nonsymmetric or non-square matrices. Our tools are bipartite graphs, matchings, and alternating paths.

Our main new result concerns LU factorization with partial pivoting. We show that if a square matrix $\mathrm{A}$ has the strong Hall property (i.e., is fully indecomposable) then an upper bound due to George and $\mathrm{Ng}$ on the nonzero structure of $\mathrm{L}$ $+U$ is as tight as possible. To show this, we prove a crucial result about alternating paths in strong Hall graphs. The alternating-paths theorem seems to be of independent interest: it can also be used to prove related results about structure prediction for $Q R$ factorization that are due to Coleman, Edenbrandt, Gilbert, Hare, Johnson, Olesky, Pothen, and van den Driessche.

Research sponsored by Applied Mathematical Sciences Research Program, U.S. DOE Office of Energy Research; by the National Science Foundation; and by the Christian Michelsen Institute.

* Xerox Palo Alto Research Center, Palo Alto, CA.

1.40

\section{AN EFFICIENT ALGORITHM TO COMPUTE ROW AND COLUMN COUNTS FOR SPARSE CHOLESKY FACTORIZATION}

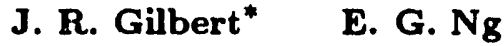 B. W. Peyton}

[Abstract of SIAM J. on Matrix Anelysis and Applications (in press); also ORNL/TM-12195, September 1992]

Let an undirected graph $G$ be given, along with a specified depth-first spanning tree $T$. We give almost-linear-time algorithms to solve the fol- lowing two problems. First, for every vertex $v$, compute the number of descendants $w$ of $v$ which some descendant of $w$ is adjacent (in $G$ ) to $v$. Second, for every vertex $v$, compute the number of ancestors of $v$ that are adjacent (in $G$ ) to at least one descendant of $v$.

These problems arise in Cholesky and $Q R$ factorizations of sparse matrices. Our algorithms can be used to determine the number of nonzero entries in each row and column of the triangular factor of a matrix from the zero/nonzero structure of the matrix. Such a prediction makes storage allocation for sparse matrix factorizations more efficient. Our algorithms run in time linear in the size of the input times a slowly-growing inverse of Ackermann's function. The best previously known algorithms for these problems ran in time linear in the sum of the nonzero counts, which is usually much larger. We give experimental results demonstrating the practical efficiency of the new algorithms.

Research sponsored by Applied Mathematical Sciences Research Program, U.S. DOE Office of Energy Research and by the Institute for Mathematics and Its Applications with funds provided by the National Science Foundation.

* Xerox Palo Alto Research Center, Palo Alto, CA.

\subsection{1}

\section{SUPERNODAL SYMBOLIC CHOLESKY FACTORIZATION OF A LOCAL. MEMORY MULTIPROCESSOR}

\section{E. $\mathbf{N g}$}

(Abstract of Parallel Computing (in press); also ORNL/TM-11836, June 1991)

In this paper, we consider the symbolic factorization step in computing the Cholesky factorization of a sparse symmetric positive definite matrix on distributed-memory multiprocessor systems. By exploiting the supernodal structure in the Cholesky factor, the performance of a previous parallel symbolic factorization algorithm is improved. Empirical tests demonstrate that there can be drastic reduction in the execution time required by the new algorithm on an Intel iPSC/ 2 hypercube.

Research sponsored by Applied Mathematical Sciences Research Program, U.S. DOE Office of Energy Research. 
1.42

\section{A SUPERNODAL CHOLESKY FACTORIZATION ALGORITHM FOR SHARED-MEMORY MULTIPROCESSORS}
E. G. $\mathbf{N g}$
B. W. Peyton

[Abstract of SIAM Journal Sci. Statist. Comput. (in press); also ORNL/TM-11814, April 1991]

This paper presents a new left-looking parallel sparse Cholesky factorization algorithm for shared-memory MIMD multiprocessors. The algo rithm is particularly well-suited for vector supercomputers with multiple processors, such as the Cray Y-MP. The new algorithm uses supernodes in the Cholesky factor to improve performance by reducing indirect addressing and memory traffic. Earlier factorization algorithms have also used supernodes in this manner. The new algorithm, however, also uses supernodes to reduce the number of system synchronization calls, often by an order of magnitude or more in practice. Experimental results on a Sequent Balance 8000 and a Cray Y-MP demonstrate the effectiveness of the new algorithm. On eight processors of a Cray Y. $M P$, the new routine performs the factorization at rates exceeding on Gflop for several test problems from the Harwell Boeing test collection, none of which are exceedingly large by current standards.

Research sponsored by Applied Mathematical Sciences Research Program, U.S. DOE Office of Energy Research.

1.43

\section{A TIGHT AND EXPLICIT REPRESENTATION OF $Q$ IN SPARSE QR FACTORIZATION}
E. G. $\mathbf{N g}$
B. W. Peyton

(Abstract of SIAM J. Matrir Analysis and Applications (in press); also ORNL/TM-12059, May 1992]

In $Q R$ factorization of a sparse $m \times n$ matrix $A(m \geq n)$ the orthogonal factor $Q$ is often stored implicitly as a lower trapezoidal matrix $H$ known as the Householder matrix. This paper presents a simple characterization of the row structure of $Q$, which could be used as the basis for a sparse data structure that can store $Q$ explicitly. The new characterization is a simple extension of a well known row-oriented characterization of the struc- ture of $H$. Hare, Johnson, Olesky, and van den Driessche have recently provided a complete sparsity analysis of the $Q R$ factorization. Let $U$ by the matrix consisting of the first $n$ columns of $Q$. Using results from Ref. 15, we show that the data structures for $H$ and $U$ resulting from our characterizations are tight when $A$ is a strong Hall matrix. We also show that $H$ and the lower trapezoidal part of $U$ have the same sparsity characterization when $A$ is strong Hall. We then show that this characterization can be e..cended to any weak Hall matrix that has been permuted into block upper triangular form. Finally, we show that permuting to block upper triangular form never increases the fill incurred during the factorization.

Research sponsored by Applied Mathematical Sciences Research Program, U.S. DOE Office of Energy Research.

\subsection{4}

\section{BLOCK SPARSE CHOLESKY ALGORITHMS ON ADVANCED UNIPROCESSOR COMPUTERS}

\author{
E. G. Ng $\quad$ B. W. Peyton
}

[Abstract of SIAM J. Sci. Statist. Comput. (in press); also ORNL/TM-11960, December 1991]

As with many other linear algebra algorithms, devising a portable implementation of sparse Cholesky factorization that performs well on the broad range of computer architectures currently available is a formidable challenge. Even after limiting our attention to machines with only one processor, as we have done in this report, there are still several interesting issues to consider. For dense matrices, it is well known that block factorization algorithms are the best means of achieving this goal. We take this approach for sparse factorization as well.

This paper has two primary goals. First, we examine two sparse Cholesky factorization algorithms, the multifrontal method and a blocked left-looking sparse Cholesky method, in a systematic and consistent fashion, both to illustrate the strengths of the blocking techniques in general and to obtain a fair evaluation of the two approaches. Second, we assess the impact of various implementation techniques on time and storage efficie rcy, paying particularly close attention to the workstorage requirement of the two methods and their variants.

Research sponsored by Applied Mathematical Sciences Research Program, U.S. DOE Office of Energy Research. 


\section{PARTITIONING A CHORDAL GRAPH INTO TRANSITTVE SUBGRAPHS FOR PARALLEL SPARSE TRIANGULAR SOLUTION}

\author{
B. W. Peyton A. Pothen* \\ X. Yuan ${ }^{\dagger}$
}

(Abstract of ORNL/TM-12270, December 1992)

A recent approach for solving sparse triangular systems of equations on massively parallel computers employs a factorization of the triangular coefficient matrix to obtain a representation of its inverse in product form. The number of general communication steps required by this approach is proportional to the number of factors in the factorization. The triangular matrix can be symmetrically permuted to minimize the number of factors over suitable classes of permutations, and thereby the complexity of the parallel algorithm can be minimized. Algorithms for minimizing the number of factors over several classes of permutations have been considered in earlier work.

Let $F=L+L^{T}$ denote the symmetric filled matrix corresponding to a Cholesky factor $L$, and let $G_{F}$ denote the adjacency graph of $F$. In this paper we consider the problem of minimizing the number of factors over all permutations which preserve the structure of $G_{F}$. The graph model of this problem is to partition the vertices $G_{F}$ into the fewest transitively closed subgraphs over all perfect elimination orderings while satisfying a certain precedence relationship. The solution to this chordal graph partitioning problem can be described by a greedy scheme which eliminates a largest permissible subgraph at each step. Further, the subgraph eliminated at each step can be characterized in terms of lengths of chordless paths in the current elimination graph. This solution relies on several results concerning transitive perfect elimination orderings introduced in this paper. We describe a partitioning algorithm with $\mathcal{O}(|V|+|E|)$ time and space complexity.

Research sponsored by Applied Mathematical Sciences Research Program, U.S. DOE Office of Energy Research; by Canadian Natural Sciences and Engineering Research Councill; and by the National Science Foundation.

*University of Waterbo, Waterloo, Ontario, Canada.

† IBM Canada Lab, North York, Ontario, Canada.
PARALLEL SPARSE CHOLESKY

FACTORIZATION ALGORITHMS FOR SHARED-MEMORY MULTIPROCESSOR SYSTEMS

\author{
E. Rothberg* \\ A. Gupta* \\ E. $\mathbf{N g}$ \\ B. W. Peyton
}

[Abstract of paper presented at the 7th International Association for Mathematios and Computer Simulation Conference on Computer Methods for Partial Differential Equations, New Brunswick, NY, June 21-24, 1992; Proc. pp. 622-628 (1992)]

In this paper, we consider sparse Cholesky factorization on a multiprocessor system that possesses a globally shared memory. Our algorithm is a parallel version of a serial blocked left-looking factorization algorithm described in Refs. 12 and 17. Unlike previous parallel left-looking algorithms, the new algorithm uses a matrix-matrix multiplication operation to implement its computationally intensive primitives. Consequently, careful implementation of these primitives enables extensive reuse of data in cache for most realistic problems, and thus reduces the volume of traffic to and from main memory. Reducing memory traffic is crucial on many shared-memory multiprocessors because the interconnect to main memory is often a serious bottleneck. We shall compare the performance of the parallel blocked algorithm with an earlier parallel left-looking algorithm studied in Ref. 13.

Research sponsored by Applied Mathematical Sciences Research Program, U.S. DOE Office of Energy Research and by the Department of Computer Science, Stanford University.

*Department of Computer Science, Stanford University, Stanford, CA. 


\section{MATHEMATICS}

\section{Applied Mathematics}

1.47

\section{PRECONDITIONED CONJUGATE GRADIENT METHODS FOR THE INCOMPRESSIBLE NAVIER-STOY.ES EQUATIONS}

\author{
P. Chin * E. F. D'Azevedo \\ P. A. Forsyth ${ }^{*}$ W.-P. Tang ${ }^{*}$
}

[Abstract of Int. J. Numer. Methods Fluids 15, 273 (1992)]

A robust technique for solving primitive variable formulations of the incompressible NavierStokes equations is to use Newton iteration for the fully implicit non-linear equations. A direct sparse matrix method can be used to solve the Jacobian but is costly for large problems; an alternative is to use an iterative matrix method. This paper investigates effective ways of using a conjugategradient-type method with an incomplete $L U$ factorization preconditioner for two-dimensional incompressible viscous flow problems. Special attention is paid to the ordering of unknowns, with emphasis on a minimum updating matrix (MUM) ordering. Numerical results are given for several test problems.

Research sponsored by Applied Mathematical Sciences Re search Program, U.S. DOE Office of Energy Research.

*University of Waterbo, Waterloo, Ontario, Canada.

1.48 tions to steady state. For high Reynold's number flows, the scaling of the variables has an important effect.

Research sponsored by Applied Mathematical Sciences Research Program, U.S. DOE Office of Energy Research.

*University of Waterlıo, Waterloo, Ontario, Canada.

\subsection{9}

\section{HYPERSINGULAR INTEGRAL EVALUATION USING MAPLE}

\section{J. Gray}

[Abstract of Maple Newsletter 8, 57 (1992)]

Hypersingular equations are an essential tool for boundary integral analysis of engineering problems. The main difficulty in employing these equations is reducing the hypersingular integral, defined in terms of a limit to the boundary, to a suitable computational form. In this note, it is shown that symbolic computation can be used to carry out the limit process, yielding exact analytic formulas for the singular integral.

Research sponsored by Applied Mathematical Sciences Research Program, U.S. DOE Office of Energy Research.

\subsection{0}

\section{BOUNDARY ELEMENT ANALYSIS AT OAK RIDGE NATIONAL LABORATORY}

\section{J. Gray}

\begin{abstract}
[Abstract of International Association for Bosndary Ele. ment Methods 5, 3 (1991)]
\end{abstract}

Boundary element analysis has proved useful for solving problems arising in research at Oak Ridge National Laboratory. These applications include electroplating simulation, fracture mechanics, fluid drop motion, and environmental modeling.

Research sponsored by Applied Mathematical Sciences Research Program, U.S. DOE Office of Energy Research. 
$1.5,1$

\section{HYPERSINGULAR INTEGRAL FORMULATION OF ELASTIC WAVE SCATTERING}

\section{J. Gray S. J. Chang*}

[Abstract of Engineering Analysis with Boundary Elements 10, 337 (1992)]

A hypersiugular boundary integral formulation for calculating two dimensional elastic wave scattering from thin bodies and cracks is described. The boundary integral equation for surface displacement is combined with the hypersingular equation for surface traction. The tifficult part in employing the traction equation, ine derivation of analytical formulas for the hypersingular integral by means of a limit to the boundary, is easily handled by means of symbolic computation. In addition, the terms containing an integrable logarithmic sirgularity are treated by straightforward numerical method, bypassing the use of Taylor series expansions. Example wave scattering calculations for cracks and thin ellipses are presented.

Research sponsored by the Exploratory Studies Program of Oak Ridge National Laboratory and by the IBM Bergen Scientific Centre, Bergen, Nor way.

* Research Reactor Dj- ision.

1.52

HYPERSINGULAR INTEGRAL SOUUTIONS AT A CORNER
L. J. Gray
L. Z. Manne*

(Abstract of paper presented at the SLAM 40th Meeting, Las Angeles, CA, July 20-24, 1992)

An efficient boundary integral analysis often requires the use of the hypersinguiar equation obtained by differentiating the standard boundary integral expression. For surface crack problems, or for nonsmooth boundaries with Dirichlet data, the hypersingular integral must be evaluated at a corner point. This paper will discuss the definition of the singular integral at a corner, and describe symbolic computation techniques for its evaluation. It is shown that Hermite interpolation provides a particularly convenient numerical approximation at a corner point. Computational results for two dimensional problems in potential theory and elasticity are presented.

Research sponsored by Applied Mathe.....ivical Sciences Research Program. U.S. DOE Office of Energy Research.

*University of Tennessee, Knoxville, TN.

1.53

\section{HERMITE INTERPOLATION ALGORITHM FOR HYPERSINGULAR BOUNDARY INTEGRALS}
L. J. Gray
C. San Soucie*

[Abstract of Int. Journal for Numerical Methods in Engineering (in press); ciso paper presented at the 13th IMACS World Congruss on Computation and Applied Mathematics, Dublin, Ireland, July 21-26, 1991, Proc. Vol. 1, pp. 2.40-241 (1991)]

A $C^{d}$ Hermite interpolation algorithm for evaluating hypersingular boundary integrals is presented. The unknown surface derivatives required by the approximation are determined by employing a tangential hypersingular equation in addition to the normal derivative equation. Test calculations for the Laplace equation in two dimensions, utilizing crack and noncrack geometries, indicate that the algorithm is a promising technique for three dimensional problems.

Research sponsored by Applied Mathematical Sciences Research Program, U.S. DOE Ofice of Energy Research.

* Louisiana State University, Baton Rouge, LA.

1.54

\section{OPTIMAL CONTROL FOR PARABOLIC SYSTEMS WITH COMPETITIVE INTERACTIONS}

\section{S. M. Lenhart V. Protopopescu}

[Abstract of Jozrnal of Mathematical Analysis and Applications (in press)]

We consider a two-dimensional parabolic system with general competitive interactions as a two-player game with conflicting objectives and with controls on the inhomogeneous (source) terms. We show the existence of an optimal solution of the game as the saddle point of a suitable objective functional.

Research sponsored by U.S. DOE Office of Energy Research. 


\section{A TWO-SIDED GAME FOR NON LOCAL COMPETITIVE SYSTEMS WITH CONTROL ON SOURCE TERMS}

\section{S. Lenhart V. Protopopescu}

\section{S. Stojanovic*}

Abstract of paper presented at the Workshop on Variational Problems, Institute for Mathemstics and lis Ap -lications, Minneapolis. MN, April 15-19, 1991; Proc. (1933); also 30th IEEE Conference on Decision and Control, Brighton, England, December 11-13. 1991; Proc. Vol. 11. pp. 18.38-1839 (1991)]

A two-sided game for itie control of a stationary semilinear competitive system with nouro:al interaction terms is considered. The controls of the system are the autonoinous sources. The saddle point of the system, i.e. the optimal solution of the game, is characterized as the unique solution of the associated optirnality system, which is solved by an iterative schene.

Research sponsured by Applied Mattiematical Sciences Research Prograrn, U.S. DOE Dtfice of Energy liesearch.

* University of Cinciastrati, Cincinnati, OH.

\subsection{6}

\section{AN OVERVIEW OF INTEGRATION METHODS FOR HYPERSINGULAR BOUNDARY INTEGRALS}

\section{E. Lutz* L. J. Gray}

\section{A. R. Ingraffea*}

(Abstract of paper presented at the Boundary Element Methods-13 Conference, Tulsa, OK, August 21-23, 1991)

Several methods of analyzing the hypersingular gradient BIE have been developed recently. This paper is a review highlighting the numerous common aspects and several differences among the methods. Significant common aspects include (a) a regularization of constant and linear terms, (b) analysis of integration points near rather than on the surface, and (c) analysis of the neigiborthood of the singular point rather than of individual elements.

Research sponsored by Applied Mathematical Sciences Research Program, U.S. DOE Office of Energy Research.

*Comell University, Ithaca, NY.

\section{INDIRECT EVALUATION OF SURFACE STRESS IN THE BOUNDARY ELEMENT ME'THOD}

\author{
$\begin{array}{ll}\text { E. Lutz* } & \text { L. J. Gray }\end{array}$ \\ A. R. Ingraffea*
}

[Abstract of paper presented at the IABEM Symposium, Rome, Italy, October 15-19, 1990; Proc. Boundary Integral Methods, pp. 339-348 (1991)]

Evaluation of stress 'on the surface' is a difficult step in the boundary element method for elasticity because it requires evaluation of hypersingular integrals. We describe an indirect method that avoids the hypersingular integral by integrating over a far surface not including the singular point or its local geometry. The derivation makes no demands for smoothness of the local surface, hence can be applied on curved surfaces and at edges. As with other derivations of hypersingular integrals, it requires continuous displacement gradients in the neighborhood of the singular point.

Research sponsored by Applied Mathematical Sciences Research Program, U.S. DOE Office of Energy Research.

*Cornell University, Ithaca, NY.

\subsection{8}

\section{THE PERFORMANCE OF A FLUID CODE ON MASSIVELY PARALLEL MACHINES}
V. E. Lynch*
J. B. Drake
P. Liewer ${ }^{\ddagger}$
B. A. Carreras ${ }^{\dagger}$
J. N. Leboeuf ${ }^{\dagger}$
D. W. Walker

(Abstract of paper presented at the International Sherwood Fusion Theory Conference, Santa Fe, NM, April 6-8, 1992)

The performance of the KITE code on the Oak Ridge Intel hypercube, the JPL Intel hypercube and Delta machine, and the TMC Connection Machine at Los Alamos will be compared with the performance and cost of other machines. With the collaboration of the JPL group, it has been possible to complete the code optimization studics on the hypercube, that is, to find the optimal number of processors for a problem of a given size. From these results, we will present the main characteristics of the next-generation hypercubes needed for plasma turbulence calculations. Tim- 
ings of convolution routines optimized for the Connection machine will also be presented.

The KITE code is an initial value, threedimensional, nonlinear, seven-field code used for plasma turbulence studies. The main components of the code are convolutions of Fourier harmonics and block tridiagonal matrix solves. Direct allocation of all arrays in the KITE code to the nodes of the Intel hypercube has allowed full use of the 128 nodes on the ORNL Intel iPSC/860 hypercube. Using this configuration and recent improvements in the compiler, we have a factor of 5 speedup over a single-processor Cray-II. The JPL Intel hypercube (64 processors, 16 Mbytes per processor) complements results of the ORNL hypercube (128 processors, 8 Mbytes per processor) by having more memory per processor. The output for the restarts and analysis of this code has been changed to a machine independent XDR binary format so we do not loose precision on the restarts and we can analyze this data on workstations from an optical disk.

Also with JPL collaboration, the first runs of this code on the Touchstone Delta machine have been made. A $25 \%$ speedup has been found for the same number of processors (128) as the ORNL hypercube. This is because of the faster communication processes in the Delta machine. A run has been made on all 512 nodes of the Delta. The speedup was a factor of 16 over a single-processor Cray-II. This run had only 2 grid points per node and 1 Fourier harmonic per node, so we are planning other runs that will be of more optimal size for 512 processors.

Research sponsored by U.S. DOE Office of Fusion Energy.

* Computing and Telecommunications Division.

†Fusion Energy Division.

$\ddagger_{\text {Jet }}$ Propulsion Laboratory, California Institute of Technology, Pasadena, CA.
1.59

\section{THREE-DIMENSIONAL FRACTURE SIMULATION WITH A SINGLE- DOMAIN, DIRECT BOUNDARY ELEMENT FORMULATION}
L. F. Martha*
A. R. Ingraffea ${ }^{\dagger}$
L. J. Gray

[Abstract of Int. J. for Num. Methods in Engineering 35, 1907 (1992)]

An efficient hypersingular boundary integral equation method for three-dimensional fracture mechanics was presented in a previous paper. The details of the numerical implementation of this method are further discussed herein. In particular, an algorithm for achieving the required differentiability of the crack surface displacement function is discussed. To illustrate the utility of the methe 1 , computational results for several strongly interacting multiple crack geometries are presented. The calculated stress intensity factors are in excellent agreement with those obtained by an approximate analytical method due to Kachanov and Laures.

Research sponsored by Applied Mathematical Sciences Research Program, U.S. DOE Office of Energy Research; by IBM Bergen Scientific Centre; and by the National Science Foundation

*University of Rio de Janeiro, Rio de Janeiro, Brazil.

${ }^{\dagger}$ Cornell University, Ithaca, NY.

1.60

\section{SIMULATION OF 3D NON-PLANAR FATIGUE CRACK GROWTH IN A TURBINE BLADE ROOT}

\author{
D. O. Potyondy* I. J. Gray \\ A. Ingraffea*
}

[Abstract of J. of the Amer. Soc. Mechanical Engineers (in press); aiso paper presented at the ASME Pressure Vessels and Piping 1992 Summer Conference, New Orleans, LA, June 21-25, 1992]

A case study of fatigue life predictions for the root of a turbine blade containing an initial flaw is presented. A fracture simulation system is utilized to perform simulations of low cycle (startup/shutdown) fatigue crack growth in the root. The turbine blade has a complex structural geometry, and the cracks have been observed to grow in a highly non-planar fashion. Simulation 
of these cracks requires the capability to model three-dimensional geometry and to allow for arbitrary non-planar crack growth. A geometric model of the portion of the root near the crack is created. Boundary conditions for this substructure are obtained from a finite element model of the entire blade (without a crack), loaded by centrifugal forces. One approach of transferring these boundary conditions to the geometric model is described. A small initial crack is added to the geometric model, and a linear elastic boundary element analysis is performed in order to compute the stress intensity factor variation (modes I, II, and III) along the crack front. The crack growth rate curve for the blade material is used along with the modeI stress intensity factor variation to predict the number of cycles, and new crack shape, for a userspecified increment of growth. The model is then locally remeshed for the next boundary element analysis. Comparisons with experimental fatigue tests of this same configuration are presented.

Research sponsored by Applied Mathematical Sciences Research Program, U.S. DOE Office of Energy Research.

*Cornell University, Ithaca, NY.

1.61

\section{LATENT HEAT THERMAL ENERGY STORAGE FOR LUNAR OXYGEN PRODUCTION}
A. D. Solomon*
G. Jacobs ${ }^{\dagger}$
V. Alexiades
M. Olszewski $\oint$

\begin{abstract}
(Abstract of paper presented at the 1992 American Society of Mechanical Engineers Annual Winter Meeting, Anaheim, CA, November 8-13, 1992; Proc. Fundamentals of Phase Change: Freezing, Melting, and Sublimation, HTDVol. 215, pp. 73-77, P. G. Kroeger and Y. Bayazitoglu, Eds. (1992)]
\end{abstract}

A necessary component of a solar-based lunar oxygen production system is a thermal energy storage module. We discuss some of the heat transfer and phase change problems associated with the design and operation of such a mod- ule based on the latent heat of melting of lunar rock.

Research sponsored by Applied Mathematical Sciences Research Program, U.S. DOE Office of Energy Research.

* Consultant, Omer, Israel.

$\dagger_{\text {Environmental Sciences Division. }}$

$\ddagger$ Office of Operational Readiness and Safety.

$\S_{\text {Engineering Technology Division. }}$

1.62

\section{A PRELIMINARY STUDY OF THE CONTROLS ON MELTING DURING IN SITU VITRIFICATION}
A. D. Solomon *
J. E. Nyquist ${ }^{\dagger}$
V. Alexiades
G. K. Jacobs ${ }^{\dagger}$
S. M. Lenhart

(Abstract of ORNL/ER-30, December 1991)

This work identified key parameters and processes in the in situ vitrification melt cycle and developed an improved understanding of ISV. Analytical approximations for several properties of molten were determined from available data. An approximation for the rate of melting versus time was derived that is consistent with data from field experiments.

Research sponsored by U.S. DOE Office of Environmental Restoration and Waste Management.

* Omer, Israel.

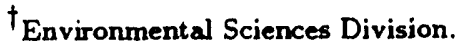




\section{Numerical Analysis}

1.63

\section{TOWARDS A COST-EFFECTIVE ILU PRECONDITIONER WITH HIGH LEVEL FILL}

\author{
E. F. D'Azevedo P. A. Forsyth* \\ W.-P. Tang*
}

[Abstract of BIT 32, 447 (1992)]

There has been increased interest in the effect of the ordering of the unknowns on Preconditioned Conjugate Gradient (PCG) methods. A recently proposed Minimum Discarded Fill (MDF) ordering technique is effective in finding good $\operatorname{ILU}(\ell)$ preconditioners, especially for problems arising from unstructured finite element grids. This algorithm can identify anisotropy in complicated physical structures and orders the unknowns in an appropriate direction. The MDF technique may be viewed as an analogue of the minimum deficiency algorithm in sparse matrix technology, and hence is expensive to compute for high level ILU $(\ell)$ preconditioners.

In this work, several less expensive variants of the MDF technique are explored to produce cost-effective ILU preconditioners. The Threshold MDF ordering combines MDF ideas with drop tolerance techniques to identify the sparsity pattern in the ILU preconditioners. The Minimum Update Matrix (MUM) ordering technique is a simplification of the MDF ordering and is an analogue of the minimum degree algorithm. The MUM ordering method is especially effective for large matrices arising from Navier-Stokes problems.

Research sponsored by Applied Mathematical Sciences Research Program, U.S. DOE Office of Energy Research.

*University of Waterloo, Waterloo, Ontario, Canada.
1.64

\section{REDUCING COMMUNICATION COSTS IN THE CONJUGATE GRADIENT ALGORITHM ON DISTRIBUTED MEMORY MULTIPROCESSORS}
E. F. D'Azevedo
C. H. Romine
(Abstract of ORNL/TM-12192, September 1992)

The standard formulation of the conjugate gradient algorithm involves two inner product computations. The results of these two inner products are needed to update the search direction and the computed solution. In a distributed memory parallel environment, the computation and subsequent distribution of these two values requires two separate communication and synchronization phases. In this paper, we present a mathematically equivalent rearrangement of the standard algorithm that reduces the number of communication phases. We give a second derivation of the modified conjugate gradient algorithm in terms of the natural relationship with the underlying Lanczos process. We also present empirical evidence of the stability of this modified algorithm.

Research sponsored by Applied Mathematical Sciences Research Program, U.S. DOE Office of Energy Research.

\subsection{5}

\section{COMPARISON OF ITERATIVE METHODS FOR NONSYMMETRIC COUPLED ELLIPTIC EQUATIONS}

\section{J. M. Donato}

(Abstract of paper presented at the SIAM 40th Anniversary Meeting, Los Angeles, CA, July 20-24, 1992)

Many interesting areas of current industry work, such as semiconductor modeling, deal with non-linear coupled systems of partial differential equations.

Here we examine iterative methods for the solution of a two-dimensional model with two coupled elliptic equations based on a linearized steady-state form of the two carrier drift-diffusion equations from semiconductor modeling. Discretizing this model system yields a large, non- 
symmetric, sparse matrix. The coupling between the two discretized elliptic equations is regulated via two coupling parameters.

Various point and block methods for a range of values of the coupling parameters are analyzed. Hybrid iterative methods, such as Alternate Block Factorization (ABF), are among the methods employed. We also employ GMRES with various preconditioners, including block and point incomplete LU factorizations.

The performance of these methods is compared. It is seen that the preferred ordering of the grid variables and the choice of iterative method are dependent upon the magnitudes of the coupling parameters. This information can then aid in the choice of iterative methods for solving more complicated, yet analogous, coupled systems.

Research sponsored by Applied Mathematical Sciences Research Program, U.S. DOE Office of Energy Research.

\subsection{6}

\section{ITERATIVE METHODS FOR NONSYMMETRIC COUPLED SYSTEMS}

\section{J. M. Donato}

(Abstract of paper presented at the Copper Mountain Conference on Iterative Methods, Copper Mountain, CO, April 9-14, 1992)

Many interesting areas of current industry work, such as semiconductor modeling, deal with non-linear coupled systems of partial differential equations.

Here we examine iterative methods for the solution of a two-dimensional model coupled system based on a linearized form of the two carrier driftdiffusion equations from semiconductor modeling. Discretizing this model system yields a large, noncymmetric, sparse matrix. The coupling between the two carrier equations is regulated via two coupling parameters.

Various point and block methods for a range of values of the coupling parameters are analyzed. Hybrid iterative methods, such as Alternate Block Factorization (ABF), are among the methods employed. We also employ GMRES with various preconditioners, including block and point incomplete LU factorizations.

The performance of these methods is compared. It is seen that the preferred ordering of the grid variables and the choice of iterative method are dependent upon the magnitudes of the coupling parameters. This information can then aid in the choice of iterative methods for solving more complicated, yet analogous, coupled systems.

Research sponsored by Applied Mathematical Sciences Research Program, U.S. DOE Office of Energy Research.

\subsection{7}

\section{THE FOURIER ANALYSIS TECHNIQUE AND €-PSEUDO-EIGENVALUES}

\section{J. M. Donato}

(Abstract of paper presented at the Copper Mountain Conference on Iterative Methods, Copper Mountain, CO, April 9-14, 1992)

The spectral radii of iteration matrices and the spectra and condition numbers of preconditioned systems are important in forecasting the convergence rates for iterative methods.

The Fourier analysis technique has been shown to be a useful method in heuristically studying the effectiveness of iterative methods by determining approximate expressions for the eigenvalues or condition numbers of matrix systems. It does not always yield a good approximation for the exact eigenvalues for non-symmetric matrices. Yet the technique works well in predicting experimental behavior of iterative methods.

For non-symmetric matrices the eigenvalues may be highly sensitive to perturbations. The spectral radii of nonsymmetric iteration matrices may not give a numerically realistic indication of the convergence of the iterative method. Trefethen and others have presented a theory on the use of $\epsilon$ pseudo-eigenvalues in the study of nonsymmetric matrix equations.

In this paper, it is shown that for Toeplitz matrices the theory of $\epsilon$-pseudo-eigenvalues includes the Fourier analysis technique as a limiting case. The Fourier eigenvalues can be viewed as an approximation to the $\epsilon$-pseudo-eigenvalues. Hence, the theory of $\epsilon$-pseudo-eigenvalues can be used in explaining the efficacy of the Fourier analysis technique.

Research sponsored by Applied Mathematical Sciences Research Program, U.S. DOE Office of Energy Research. 
1.68

\section{THE RELATIONSHIP BETWEEN $\epsilon$-PSEUDO-EIGENVALUES AND THE FOURIER ANALYSIS TECHNIQUES}

\section{J. M. Donato}

(Abstract of paper presented at the SIAM 40th Anniversary Meeting, Los Angeles, CA, July 20-24, 1992)

The spectral radii of iteration matrices and the spectra and condition numbers of preconditioned systems are important in forecasting the convergence rates for iterative methods.

For non-symmetric matrices the eigenvalues may be highly sensitive to perturbations. The spectral radii of nonsymmetric iteration matrices may not give a numerically realistic indication of the convergence of the iterative method. Trefethen and others have presented a theory on the use of $\epsilon$ pseudo-eigenvalues in the study of nonsymmetric matrix equations.

The Fourier analysis technique has been a useful tool in studying the effectiveness of iterative methods by determining approximate expressions for the eigenvalues or condition numbers of matrix systerns. While it does not always yield a good approximation for the exact eigenvalues for non-symmetric matrices, the technique works well in predicting experimental behavior of iterative methods.

In this paper, it is shown that for Toeplitz matrices the theory of $\epsilon$-pseudo-eigenvalues includes the Fourier analysis technique as a limiting case. The Fourier eigenvalues can be viewed as an approximation to the $\epsilon$-pseudo-eigenvalues. Hence, the theory of $\epsilon$-pseudo-eigenvalues can be used in explaining the efficacy of the Fourier analysis technique for analyzing systems where the coefficient matrix is Toeplitz.

Research sponsored by Applied Mathematical Sciences Research Program, U.S. DOE Office of Energy Research.
1.69

\section{BOUNDARY ELEMENTS ON DISTRIBUTED MEMORY ARCHITECTURES}

\author{
R. E. Flanery J. B. Drake \\ L. J. Gray
}

[Abstract of paper presented at the IABEM-2 (International Association for Boundary Elements Meeting), Boulder, CO, August 2-5, 1992]

The modeling of the scattering and transmission of elastic waves will, in general, give rise to large scale computations. Transient problems are inherently computationally intensive and frequency domain solutions can, depending upon the scattering geometry, involve a large number of unknowns. It is therefore desirable to investigate parallel algorithms for elastodynamic modeling.

A previous parallel boundary element algorithm for Laplace's equation employed a row oriented decomposition strategy. Each processor of a distributed memory parallel computer was responsible for constructing specified rows of the coefficient matrix, and for manipulating these rows in the factorization and solve routines. There are two significant drawbacks to this approach. First, if the number of rows is not a multiple of the number of processors, the load balancing will suffer, and second, each processor must have knowledge of the complete geometry. As the local memory is usually somewhat limited, this can potentially be a problem for large scale calculations.

Research sponsored by Applied Mathematical Sciences Research Program, U.S. DOE Office of Energy Research.

\subsection{0}

\section{TWO VARIANTS OF MINIMUM DISCARDED FILL ORDERING}

\section{P. A. Forsyth $\quad$ W.-P. Tang* \\ E. F. D'Azevedo}

[Abstract of paper presented at the IMACS International Symposium on Iterative Methods in Linear Algebra, Brussels, Belgium, April 2-5, 1991; Proc. pp. 603-612 (1991)]

It is well known that the ordering of the unknowns can have a significant effect on the convergence of Preconditioned Conjugate Gradient (PCG) methods. There has been considerable experimental work on the effects of ordering for reg- 
ular finite difference problems. In many cases, good results have been obtained with preconditioners based on diagonal, spiral or natural row orderings. However, for finite element problems having unstructured grids or grids generated by a local refinement approach, it is difficult to define many of the orderings used for more regular problems. A recently proposed Minimum Discarded Fill (MDF) ordering technique is effective in finding high quality Incomplete LU (ILU) preconditioners, especially for problems arising from unstructured finite element grids. Testing indicates this algorithm can identify a rather complicated physical structure in an anisotropic problem and orders the unknowns in the "preferred" direction. The MDF technique may be viewed as the numerical analogue of the minimum deficiency algorithm in sparse matrix technology. At any stage of the partial elimination, the MDF technique chooses the next pivot node so as to minimize the amount of discarded fill, and hence the MDF ordering is expensive to compute for high-level ILU preconditioners.

In this work, two efficient variants of the MDF technique are explored to produce cost-effective high-order ILU preconditioners. The Threshold MDF orderings combine MDF ideas with drop tolerance techniques to identify the sparsity pattern in the ILU preconditioners. These techniques identify an ordering that encourages fast decay of the entries in the ILU factorization. The Minimum Update Matrix (MUM) ordering technique is a simplification of the MDF ordering and is closely related to the minimum degree algorithm. The MUM ordering is especially effective for large problems arising from Navier-Stokes problems. Some interesting pictures of the orderings are presented using a visualization tool developed at the University of Waterloo.

Research sponsored by the Natural Sciences and Engineering Research Council of Canada; by the Information Technology Research Centre, Province of Ontario; and by Applied Mathematical Sciences Research Program, U.S. DOE Office of Energy Research.

*University of Waterloo, Waterioo, Ontario, Canada.
1.71

\section{AN IMPLEMENTATION OF A PARALLEL MOL SOLVER ON THE INTEL GAMMA PARALLEL COMPUTER}

\author{
W. F. Lawkins J. S. Payne*
}

[Abstract of paper presented at the 7th International Association for Mathematics and Computer Simulation Conference on Computer Methods for Partial Differential Equations, New Brunswick, NJ, June 21-24, 1992; Proc. (1992)]

An implicit parallel method-of-lines solver that has been implemented on the MIMD Intel Gamma prototype supercomputer is discussed. The strategy for implementation is to execute the ODE solver sequentially and to do the numerical linear algebra in parallel. Performance studies for this implementation are presented.

Research sponsored by Applied Mathematical Sciences Research Program, U.S. DOE Office of Energy Research.

*Computing and Telecommunications Division.

\subsection{2}

\section{THE HIERARCHICAL SPATIAL DECOMPOSITION OF THREE- DIMENSIONAL PARTICLE-IN- CELL PLASMA SIMULATIONS ON MIMD DISTRIBUTED MEMORY MULTIPROCESSORS}

\author{
D. W. Walker
}

(Abstract of ORNL/TM-12071, July 1992)

The hierarchical spatial decomposition method is a promising approach to decomposing the particles and computational grid in parallel particle-in-cell application codes, since it is able to maintain approximate dynamic load balance while keeping communication costs low. In this paper we investigate issues in implementing a hierarchical spatial decomposition on a hypercube multiprocessor. Particle attention is focused on the communication needed to update guard ring data, and on the load balancing method. The hierarchical approach is compared with other dynamic load balancing schemes.

Research sponsored by Applied Mathematical Sciences Research Program, U.S. DOE Office of Energy Research. 
1.73

\section{A FORTRAN 90 CODE FOR MAGNETOHYDRODYNAMICS, PART I: BANDED CONVOLUTION}

\author{
D. W. Walker
}

(Abstract of ORNL/TM-12032, March 1992)

This report describes progress in developing a Fortran 90 version of the KITE code for studying plasma instabilities in Tokamaks. In particu- lar, the evaluation of convolution terms appearing in the numerical solution is discussed, and timing results are presented for runs performed on an $8 \mathrm{k}$ processor Connection Machine (CM-2). Estimates of the performance on a full-size $64 \mathrm{k} \mathrm{CM}-2$ are given, and range between 100 and 200 Mflops. The advantages of having a Fortran 90 version of the KITE code are stressed, and the future use of such a code on the newly announced CM5 and Paragon computers, from Thinking Machines Corporation and Intel, is considered.

Research sponsored by U.S. DOE Office of Fusion Energy.

\section{Global Climate Modeling}

\subsection{4}

\section{ATMOSPHERIC SPECTRAL MODELS ON MIMD AND SIMD ARCHITECTURES}

\author{
J. B. Drake D. W. Walker \\ P. H. Worley
}

(Abstract of paper presented at the Minisymposium on Computing Climate Change: Can We Beat Nature? Supercomputing ' 91 , Albuquerque, NM, November 21, 1991)

The spectral transform method is one of the standard numerical techniques for solving partial differential equations on the sphere and has found wide application in global climate inodeling. This paper describes a parallel algorithm for the spectral transform method and its implementation on the Intel-iPSC/860 in the context of a full climate code, NCAR's Community Climate Model, CCM2.

The spectral transform method involves the transformation of data between the physical, Fourier and spectral domains. Fourier transforms in the longitude direction are followed by summation in the latitude direction to evaluate the discrete spectral transform. A simple way of parallelizing the spectral method is to decompose the physical problem domain in just the latitude direction. This allows an optimized sequential FFT algorithm to be used in the longitude direction. However, this approach limits the number of processors that can be effectively utilized in a simulation. Decomposing the problem in both directions allows the parallelism inherent in the problem to be exploited more effectively. The two dimensional decomposition over longitude and latitude requires a parallel FFT as well as a parallel Legendre transform, but maximizes the parallelism of the computation in the vertical direction. Since the vertical coupling of climate models is most severe and involves the dominant computational load, very effective parallel implementations can be achieved.

Research sponsored by U.S. DOE Office of Health and Environmental Research.

\subsection{5}

\section{PARALLEL ALGORITHMS FOR THE SPECTRAL TRANSFORM AND SEMI-LAGRANGIAN TRANSPORT METHODS}

\author{
J. B. Drake D. L. Williamson* \\ I. Foster ${ }^{\dagger}$
}

(Abstract of paper presented at the Computer Hardware Advanced Mathematics and Model Physics Science Team Meeting, Las Vegas, NV, March 17, 1992)

Our work involves the use of parallel supercomputers to increase the throughput of climate simulations. The research seeks to develop efficient parallel algorithms for the spectral transform method and the semi-Lagrangian transport method used in the Community Climate Model. In addition it seeks to provide variants of these un- 
derlying algorithms that have superior serial and parallel efficiencies. The following tasks are included:

- introduction of finer grain parallelism in the MIMD spectral transform algorithrn,

- improvement of the parallel FFT used for the MIMD spectral transform method,

- development of communication algorithms for an efficient, scalable spectral transform on data parallel computer architectures,

- research on the semi-Lagrangian transport method for climate modeling on parallel computing platforms,

- research on isotropic transform grids for the spectral transform method on parallel computing platforms.

Research sponsored by Applied Mathematical Sciences Re search Program, U.S. DOE Office of Energy Research.

*National Center for Atmospheric Research, Boulder, CO.

† Argonne National Laboratory, Argonne, IL.

\subsection{6}

\section{PARALLELIZING THE SPECTRAL TRANSFORM METHOD - PART II}

\section{W. Walker P. H. Worley \\ J. B. Drake}

(Abstract of Concurrency: Practice and Experience 4(7), 509 (1992); also ORNL/TM-11855, July 1991]

The spectral transform method is a widelyused numerical technique for solving partial differential equations on the sphere in global climate modeling. This paper describes the parallelization and performance of the spectral method for solving the nonlinear shallow water equations on the surface of a sphere using a 128-node Intel iPSC/860 hypercube. Solving the shallow water equations represents a computational kernel of more complex climate models. This work is part of a research program to develop climate models that are capable of much longer simulations at a signif- icantly finer resolution than current models. Such models are important in underst anding the effects of the increasing atmospheric concentrations of greenhouse gases, and the computational requirements are so large that massively parallel multiprocessors will be necessary to run climate models simulations in a reasonable amount of time.

The spectral method involves the transformation of data between the physical, Fourier, and spectral domains. Each of these domains is two-dimensional. The spectral method performs Fourier transforms in the longitude direction followed by summation in the latitude direction to evaluate the discrete spectral transform. A simple way of parallelizing the spectral code is to decompose the physical problem domain in just the latitude direction. This allows an optimized sequential FFT algorithm to be used in the longitude direction. However, this approach limits the number of processors that can be brought to bear on the problem. Decomposing the problem over both directions allows the parallelism inherent in the problem to be exploited more effectively - the grain size is reduced, so that more processors can be used.

Results are presented that show that decomposing over both directions does result in a more rapid solution of the problem. The results show that for a given problem and number of processors, the optimum decomposition has approximately equal numbers of processors in each direction. Load imbalance also has an impact on the performance of the method. The importance of minimizing communication latency and overlapping communication with calculation is stressed. General methods for doing this, that may be applied to many other problems, are discussed.

Research sponsored by Applied Mathematical Sciences Research Program, U.S. DOE Office of Energy Research. 


\section{A STANDARD TEST SET FOR NUMERICAL APPROXIMATIONS TO THE SHALLOW WATER EQUATIONS IN SPHERICAL GEOMETRY}

\author{
D. L. Williamson* \\ J. B. Drake \\ J. J. Hack* \\ R. Jakob* \\ P. N. Swarztrauber ${ }^{*}$
}

(Abstract of ORNL/TM-11895, August 1991)

A suite of seven test cases is proposed for the evaluation of numerical methods intended for the solution of the shallow water equations in spherical geometry. The shallow water equations exhibit the major difficulties associated with the horizontal dynamical aspects of atmospheric modeling on the spherical earth. These cases are designed for use in the evaluation of numerical methods proposed for climate modeling and to identify the potential trade-offs which must always be made in numerical modeling. Before a proposed scheme is applied to a full baroclinic atmospheric model it must perform well on these problems in comparison with other currently accepted numerical methods. The cases are presented in order of complexity. They consist of advection across the poles, steady state geostrophically balanced flow of both global and local scales, forced nonlinear advection of an isolated low, zonal flow impinging on an isolated mountain, Rossby-Haurwitz waves and observed atmospheric states. One of the cases is also identified as a computer performance/algorithm efficiency benchmark for assessing the performance of algorithms adapted to massively parallel computers.

Research sponsored by U.S. DOE Office of Health and Environmental Research.

* National Center for Atmospheric Research, Boulder, CO.

\section{PARALLELIZING THE SPECTRAL TRANSFORM METHOD}
P. H. Worley
J. B. Drake

(Abstract of Concurrency: Practice and Experience 4(4), 269 (1992); also paper presented at the 6th Distributed Memory Computing Conference, Portland, OR, April 28May 2, 1991; Proc. pp. 306-313 (1991)]

The spectral transform method is a standard numerical technique used to solve partial differential equations on the sphere in global climate modeling. In particular, it is used in CCM1 and CCM2, the Community Climate Models developed at the National Center for Atmospheric Research. This paper describes initial experiences in parallelizing a program that uses the spectral transform method to solve the nonlinear shallow water equations on the sphere, showing that an efficient implementation is possible on the Intel iPSC/860. The use of PICL, a portable instrumented communication library, and ParaGraph, a performance visualization tool, in tuning the implementation is also described.

The Legendre transform and the Fourier transform comprise the computational kernel of the spectral transform method. This paper is a case study of parallelizing the Legendre transform. For many problem sizes and numbers of processors, the spectral transform method can be parallelized efficiently by parallelizing only the Legendre transform.

Research sponsored by Applied Mathematical Sciences Research Program, U.S. DOE Office of Energy Research.

\subsection{9}

\section{A MATHEMATICAL MODEL FOR RADIATION-INDUCED MYELOPOIESIS}

\author{
T. D. Jones* M. D. Morris \\ R. W. Young ${ }^{\dagger}$
}

[Abstract of Radiation Research 128, 258 (1991)]

A model for damage, repair, killing, and repopulation of myelopoietic marrow is presented. Evaluation produces time and dose-rate profiles during and following any complex irradiation. Equations model variable dose rates, multiple ex- 
posure, different sources, and arbitrary intervals between treatments. If factors which dominate the control of biological processes can be demonstrated, an option is to set biological rate constants to experimentally determined values. Previously, knowledge did not permit identification of dominating biological processes and their temporal rates. But a unique feature of this study is that unspecified lesions for killing and injury of cells are evaluated from mortality data on the animal species of choice. "Unspecified" is used to indicate a condition of assumption-free modeling of molecular processes, whereby rate constants for cellular effects are simply computed directly from animal mortality data. Coefficients (estimated by maximum-likelihood methods for nonspecific pro- cesses) are compared with experimental values for specific processes. The model has many uses, including modeling of the myelopoietic potential as a function of time. Another option is to calculate the whole-body survival curve for cells that control myelopoiesis as a result of the treatment schedule. Also through simple extensions of the model, an extremely complex protocol can be identified with an equivalent prompt dose value-even for partialbody, fractionated exposures.

Research sponsored by the Defense Nuclear Agency and the U.S. Department of Energy.

* Health and Safety Research Division.

'Defense Nuclear Agency, Washington, DC.

\section{STATISTICS}

\section{Design and Analysis of Computational Experiments}

\subsection{0}

\section{BAYESIAN DESIGN AND ANALYSIS OF COMPUTER EXPERIMENTS: TWO EXAMPLES}

\section{T. J. Mitchell \\ M. D. Morris}

[Abstract of Statistica Sinica 2, 359 (1992)]

In a computer experiment, the data are produced by a computer program that models a physical system. The experiment consists of a set of model runs; the design of the experiment specifies the choice of program inputs for each run. This report demonstrates two applications of a Bayesian method for the design and analysis of computer experiments (DACE) to predict model output corresponding to input values for which the model has not been run. When the original code is long-running, the fast predictor produced by this method can serve as an efficient, though approximate, substitute. The models used in the two examples are (i) a computer model for the combustion of methane and (ii) a computer model that simulates the compression molding of sheet molding compound (SMC) in the manufacture of an automobile hood.

Research sponsored by Applied Mathematical Sciences Research Program, U.S. DOE Office of Energy Research.

\subsection{1}

\section{USING COMPUTER EXPERIMENTS TO CONSTRUCT A CHEAP SUBSTITUTE FOR AN EXPENSIVE SIMULATION MODEL}

\section{T. J. Mitchell M. D. Morris}

(Abstract of paper presented at the Computing Science and Statistics: Interface 1991, Seattle, WA, April 22-24, 1991; Proc. pp. 272-277 (1991)]

There is widespread use of computer models as tools in scientific research. As surrogates for physical or behavioral systems, such models 
can be subjected to experimentation, the goal being to predict how the corresponding real system would behave under certain conditions. For longrunning (expensive) model codes, there may be a severe limitation on the number of experiments that can reasonably be done. This motivates the construction of a fast-running (cheap) approximation to the original code, for use in experiments where a large number of runs may be necessary. Here we discuss our approximation of a simulation model for the compression molding of sheet molding compound, applied to the manufacture of an automobile hood. The approximation was constructed using Bayesian interpolation methods for prediction of the movement of the flow front. The predictions were based on data generated by a sequence of computer experiments, using designs chosen according to a type of D-optimality criterion.

Research sponsored by Applied Mathematical Sciences Research Program, U.S. DOE Office of Energy Research.

\subsection{2}

\section{THE SPATIAL CORRELATION FUNCTION APPROACH TO RESPONSE SURFACE ESTIMATION}

\section{T. J. Mitchell M. D. Morris}

(Abstract of paper presented at the 1992 Winter Simulation Conference, Arlington, VA, December 13-16, 1992)

This is an expository paper which discusses an alternative to conventional response surface methodology for use in simulation experiments where the objective is to express an output variable (response) as a function of several input variables. The method is Bayesian in the sense that uncertainty about the true response function $y$ is expressed by the random function $Y$, defined on the region of interest $X$ in the space of the input parameters. If $Y$ is Gaussian, straightforward formulas exist for updating $Y$ given observations of $y\left(x^{1}\right), y\left(x^{2}\right), \cdots, y\left(x^{n}\right)$, which are available from $n$ simulation runs at different settings $\left(x^{i} \in X\right)$ of the input parameters. The posterior mean of $Y(x)$, viewed as a function of $x$, serves as the estimated response function $\hat{\mathbf{y}}$. The method is driven primarily by means of a chosen spatial correlation function $(S C F)$, which defines the prior correla- tion between the responses at any two points in the space of the input parameters. Once the SCF is chosen, the method is naturally adaptive $-\hat{y}$ becomes more subtle and complex as more simulation runs are made, with no intervention required to add terms to a parametric model. Although much of the focus of this paper is on deterministic simulations, where we have had most of our experience, we shall show how modifications can be made to handle "random" responses. Some examples are discussed to illustrate the ideas and the nature of the results.

Research sponsored by Applied Mathematical Sciences Research Program, U.S. DOE Office of Energy Research.

\subsection{3}

\section{EXPLORATORY DESIGNS FOR COMPUTATIONAL EXPERIMENTS}

\author{
M. D. Morris T. J. Mitchell
}

[Abstract of ORNL/TM-12045, October 1992; also paper preserited at the Institute for Mathematical Statistics Special Topics Meeting on Industrial Statistics, Philadelphia PA, June 10-12, 1991; Proc. IMS Bulletin (1991)]

Recent work by Johnson, Moore, and Ylvisaker (1990) establishes equivalence of the maximin distance design criterion and an entropy criterion motivated by function prediction in a Bayesian setting. The latter criterion has been used by Currin, Mitchell, Morris, and Ylvisaker (1991) to design experiments for which the motivating application is approximation of a complex deterministic computer model. Because computer experiments often have a large number of controlled variables (inputs), maximin designs of moderate size are often concentrated in the corners of the cuboidal design region, i.e. each input is represented at only two levels. Here we will examine some maximin distance designs constructed within the class of Latin hypercube arrangements. The goal of this is to find designs which offer a compromise between the entropy/maximin criterion, and good projective properties in each dimension (as guaranteed by Latin hypercubes). A simulated annealing search algorithm is presented for constructing these designs, and patterns apparent in the optimal designs are discussed.

Research sponsored by Applied Mathematical Sciences Research Program, U.S. DOE Office of Energy Research. 
1.84

\section{BAYESIAN DESIGN AND ANALYSIS OF COMPUTER EXPERIMENTS: USE OF DERIVATIVES IN SURFACE PREDICTION}

\section{D. Morris T. J. Mitchell D. Ylvisaker ${ }^{*}$}

(Abstract of Technometrics (in press); also ORNL/TM11699, June 1991; also paper presented at the 1991 Joint Statistical Meetings, Atlanta, GA, August 19-22, 1991]

The work of Currin et al. (1988) and others in developing "fast predictive approximations" of computer models is extended for the case in which derivatives of the output variable of interest with respect to input variables are available. In addition to describing the calculations required for the Bayesian analysis, the issue of experimental design is also discussed, and an algorithm is described for constructing "maximin distance" designs. An example is given based on a demonstration model of eight inputs and one output, in which predictions based on a maximin design, a Latin hypercube design, and two "compromise" designs are evaluated and compared.

Research sponsored by Applied Mathematical Sciences Research Program, U.S. DOE Office of Energy Research.

*University of California, Los Angeles, CA.

\section{Computational Distribution Theory}

\subsection{5}

\section{THE NEGATIVE HYPERGEOMETRIC DISTRIBUTION AND ESTIMATION BY MOMENTS}

\section{K. O. Bowman M. A. Kastenbaum*} L. R. Shenton ${ }^{\dagger}$

[Abstract of Commun. Statist. Simula. 21(2), 301 (1992)]

An exact evaluation of the joint efficiency of the method of moments is given, along with a study of properties of the moment estimation for the basic beta distribution parameters. Series are derived for the first four moments of the moment estimators; summation techniques are required for evaluations, and simulation approaches are used for validation.

Research sponsored by U.S. DOE and by the Center for Indoor Air Research.

*Fort Myers, FL.

†University of Georgia, Athens, GA.
1.86

THE USE OF MOMENT ESTIMATORS TO DETERMINE THE PARAMETERS OF CONCENTRATION FLUCTUATIONS IN RANDOM AREA ATOM PROBE ANALYSES

\section{K. O. Bowman M. K. Miller* \\ L. R. Shenton ${ }^{\dagger}$}

[Abstract of Sufface Science (in press); also paper presented at the 39th International Field Emission Symposium, Halifax, Canada, August 10-14, 1992]

A method based on moment estimators for the estimation of the extent of concentration fluctuations in atom probe random area analyses is described. The extent of the concentration fluctuations determined from this moment estimators method and from the maximum likelihood $\mathrm{P}_{a}$ method showed excellent agreement for a series of random area analyses obtained from a spinodally decomposed $\mathrm{Fe}-45 \% \mathrm{Cr}$ alloy that was aged at $500^{\circ} \mathrm{C}$ within the low temperature miscibility gap.

Research sponsored by Division of Materials Sciences, U.S. DOE.

* Metals and Ceramics Division.

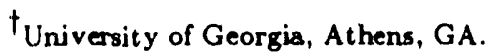


1.87

\section{PARAMETER ESTIMATION FOR THE BETA DISTRIBUTION}
K. O. Bowman
L. R. Shenton*

[Abstract of J. Statist. Comput. Simul. 43, 217 (1992)]

Moment estimators, based on the first two sample moments, for the two index parameters of the beta density (known end-points) are studicd. Four moments of these estimators are set up using Computer Oriented Extended Taylor Series (COETS) to 60 terms followed by rational fraction approximations. These indicate, over a limited parameter space, that allowing for simplicity of calculation and other characteristics they are preferable to maximum likelihood estimators.

Research sponsored by Applied Mathematical Sciences Research P: ogram, U.S. DOE Office of Energy Research.

*University of Georgia, Athens, GA.
1.88

\section{MIXTURES OF LOGARITHMIC DISTRIBUTIONS}

\author{
K. O. Bowman L. R. Shenton* \\ M. A. Kastenbaum ${ }^{\dagger}$ \\ [Abstract of IMS Bulletin 20, 239 (1991)]
}

In the abstract $90 t-37$, we introduced mixtures of binomial distributions derived by assuming that the probability parameter $p$ varies according to some law. We used the transformation $p=\exp (-t)$ and considered various appropriate densities for the transformed variables. It turns out that the Laplace transform is now the fundamental entity. Large numbers of new binomial mixtures are generated in this way. The transformation may involve several variates leading to "multivariate" binomial mixtures. An extension of this to the logarithmic distribution with parameter $p$ is possible. Frullani integrals and Laplace transforms are encountered.

Research sponsored by U.S. DOE and by the Center for Indoor Air Research.

*University of Georgia, Athens, GA.

$\dagger_{\text {Fort Myers, FL. }}$

\section{Research in General Statistical Methods}

\subsection{9}

\section{OVERDISPERSION: NOTES ON DISCRETE DISTRIBUTIONS}

\author{
K. O. Bowman \\ M. A. Kastenbaum ${ }^{\dagger}$ \\ L. R. Shenton*
}

(Abstract of ORNL/TM-12167, Septemt I 1992)

We introduce mixtures of binomial dis'ributions derived by assuming that the prot at:lity parameter $p$ varies accord 6 to some law. We use the transformation $p=\exp (-t)$ and consider various arpropriate densities for the transformed variables. In the prccess, the Laplace transform becomes the fundamental entity. Large numbers of new binomial mixtures are generated in this way. Some transformations may involve several variates that lead to "multivariate" binomial mixtures. An extension of this to the logarithmic distribution, with parameter $p$, is possible. Frullani integrals and Laplace transforms are encountered.

Graphical representations of some of the more significant distributions are given. These include probability functions, regions of validity, and three dimensional representations ui probability functions showing the response to variation of parameters when two parameters are involved.

Research sponsored by the Center for Indoor Air and Research and U.S. DOE.

*University of Georgia, Athens, GA.

$t_{\text {Ft. Myers, }}$ LL. 


\subsection{0}

\section{DISCRETE PEARSON DISTRIBUTIONS}

\section{K. O. Bowman L. R. Shenton* M. A. Kastenbaum ${ }^{\dagger}$}

(Abstract of Institute of Mathematical Statistics Bulletin 20, 410 (1991); also Journal of Computational and Graph. ical Statistics (in press); also ORNL/TM-11899, November 1991]

These distributions are generated by a first order recursive scheme which equates the ratio of successive probabilities to the ratio of two corresponding quadratics. The use of a linearized form of this model will produce equations in the unknowns matched by an appropriate set of moments (assumed to exist). Given the moments we may find valid solutions. There are two cases; (a) distributions defined on the ron-negative integers (fnite or infinite) and (b) distributions defined on negative integers as well. For (a) given the first four moments, it is possible to set this up as equations of finite or infinite degree in the probability of a zero occurrence, the $s$ the component being a product of $s$ ratios of linear forms in this probability. For (b) the equation for the zero probability is purely linear but may involve slowly converging series; here a particular case is the discrete normal. Regions of validity are being studied.

Research sponsored by U.S. DOE and by the Center for Indoor Air Research.

*University of Georgia, Athens, GA.

†Fort Myers, FL.

1.91

\section{ASYMPTOTICALLY VALID PREDICTION INTERVALS FOR LINEAR MODELS}

\section{R. L. Schmoyer}

[Abstract of Technometrics 34(4), 399 (1992)]

For estimating the parameters of a linear model, whether by least squares or many robust alternativs, one can use confidence limits that are based on large-sample normal approximations. These limits are asymptotically valid for a wide range of error distributions. Unfortunately, asymptotic validity does not automatically hold for prediction limits for a new observation, because they depend on both parameter estimates and the underlying error distribution. Resampling methods provide prediction limits that are asymptotically valid, but they tend to be computationally intensive, especially when used with robust estimators. This article presents alternative prediciion limits that are asymptotically valid, are not based on resampling, and are computationally more tractable than methods that are. They are based on quantiles of a convolution of the empirical distribution of the residuals and the limiting normal distribution of the parameter estimates. Simulations suggest that the proposed limits perform reasonably and are comparable to limits based on reampling.

Research sponsored by U.S. DOE Office of Health and Environmental Research.

1.92

\section{OVERDISPERSION OF AGGREGATED GENETIC DATA}

\section{K. O. Bowman M. A. Kastenbaum*}

[Abstract of Mutation Research (in press)]

Aggregation of sampled Poisson data clusters results in overdispersed data sets whose variances exceed their means. Such mixtures of Poissons are shown to result in the negative binomial distribution, under certain conditions. When applied to aggregated data on spontaneous aberrations in peripheral lymphocytes from normal human beings, the negative binomial does very well at characterizing the observed relative frequencies. A mixture of binomials that reduces to the beta-binomial is also discussed. Both distributions are characterized by two parameters that may be estimated, in closed-form, from the respective estimates of the sample mean and variance.

Research sponsored by U.S. DOE Center for Indoor Air Research.

*Bayse, VA. 
1.93

\section{SOFTWARE CONSIDERATIONS FOR RELIABILITY CENTERED MAINTENANCE ANALYSES}

\author{
D. M. Flanagan
}

(Abstract of ORNL/M-1530, July 1991)

Software needs and some software tools for reliability, maintainability, and availability are considered for a Reliability Centered Maintenance pilot study for the Y-12 Facility operated by the Department of Energy in Oak Ridge, Tennessee. This initial study recommends software tools and issues to be considered further.

Research sponsored by Applied Mathematical Sciences Research Program, U.S. DOE Office of Energy Research.

\subsection{4}

\section{COMPARISON OF MODELS FOR DECONVOLUTING THE COMPOSITIONS OF COEXISTING PHASES}

\author{
M. K. Miller* \\ A. Cerezo ${ }^{\dagger}$ \\ K. O. Bowman \\ J. M. Hyde ${ }^{\dagger}$
}

[Abstract of Applied Surface Science (in press); also paper presented at the 39th International Field Emission Symposium, Halifax, Canada, August 10-14, 1992]

A comparison has been performed on the best fits between frequency distributions obtained from atom probe random area analyses and probability densities obtained from 1) the sinusoidal or $\mathrm{P}_{a}$ model, 2) a mixture of two binomial distributions, and 3) mixtures of two normal distributions. The materials used for this comparison were $\mathrm{Fe}-24 \% \mathrm{Cr}$ and $\mathrm{Fe}-45 \% \mathrm{Cr}$ alloys aged between 4 and $500 \mathrm{~h}$ at $500^{\circ} \mathrm{C}$ to produce a mixture of an iron-rich $\alpha$ phase and a chromium-enriched $\alpha^{\prime}$ phase. The modified three parameter Gaussian model provided satisfactory fits for both materials and all aging conditions.

Research sponsored by U.S. DOE Office of Materials Sciences.

*Metals and Ceramics Division.

$t_{\text {University of Oxford, Oxford, UK. }}$
1.95

\section{HMODEL: AN X TOOL FOR GLOBAL MODEL SEARCH}

\section{G. Ostrouchov}

Abstract of paper presented at the 10th Symposium on Computatiunal Statistics, Neuchatel, Switzerland, August 23-28, 1992; Proc. Computational Statistics, Vol. 1, pp. 269-274, Y. Dodge and J. Whittaker, Eds., Physica Verlag (1992)]

HModel is a global model search tool that has $\mathrm{X}$ window graphics capability. The user defines the largest generalized polynomial model to consider, HModel generates the collection of all smaller hierarchical models that it contains and displays a graphical representation of the collection. Then, given a model optimality criterion threshold HModel finds all models that are at least as good. That is, HModel classifies all models into two groups: those at or below the threshold and those above the threshold. If the set of models at least as good as the threshold is small, finding this set requires fitting only a small fraction of all models. The selection of models to fit can be automatic or conveniently controlled by the user. A search string facility for selecting sets of models as well as other set operations are also available. All of these functions interact with the graphical representation of the collection of models. The current implementation and this paper focus on log-linear models for categorical data and the Akaike and Bayes information criteria although the underlying ideas are more general. The search can manipulate a collection of up to about 200,000 models on a high performance workstation. For example, six categorical variables give 140,035 possible models of order three or less.

Research sponsored by U.S. Department of Energy. 


\section{Statistical Sampling Theory}

1.96

\section{STATISTICAL DESIGN FOR SAMPLING AND ANALYSIS OF ANIMAL POPULATIONS FOR CHEMICAL CONTAMINATION}

\section{J. J. Beauchamp \\ J. F. McCarthy* \\ D. Rosenblatt ${ }^{\dagger}$ \\ L. R. Shugart*}

[Abstract of Risk Analysis 12(2), 233 (1992)]

A strategy for sampling of animal tissues and a statistical approach for analyzing data on body burdens of a parent chemical and its metabolites is presented such that the data may be evaluated in relation to the detection limit(s) of the analytical techniques used and the criterion levels established for acceptable tissue concentrations.

Research sponsored by the U.S. Army Biomedical Research and Development Laboratory and U.S. DOE.

*Environmental Sciences Division.

$\dagger_{\text {U.S. Army Biomedical Research and Development Labo }}$ ratory, Fort Detridk, MD.

\subsection{7}

\section{A STUDY OF INTERSTATE MOTOR CARRIER VEHICLE MILES OF TRAVEL}

\section{P. S. Hu* T. Wright S.-P. Miaou ${ }^{\dagger} \quad$ R. Gorman ${ }^{\ddagger}$} S. C. Davis ${ }^{\dagger}$

[Abstract of Transpn. Res.-A 25A(6), 451 (1991)]

This article summarizes the evaluation results of six data sources in terms of their ability to estimate the number of commercial trucks operating in interstate commerce and their vehicle miles of travel by carrier type and by state. The six data sources were: (a) Truck Inventory and Use Survey of the U.S. Bureau of the Census; (b) Nationwide Truck Activity and Commodity Survey of the U.S. Bureau of the Census; (c) National Truck Trip Information Survey of the University of Michigan Transportation Research Institute; (d) Highway Performance Monitoring Sys- tem of the Federal Highway Administration, U.S. Department of Transportation; (e) International Registration Plan of the American Association of Motor Vehicle Administrators; and (f) State fuel tax reports from each individual state and the International Fuel Tax Agreement. Evaluation results concluded that none of the data sources by themselves were capable of providing reliable estimates at the same level. Although several attempts were made to combine the strengths of different data sources so that reliable estimates could be generated, none of the were successful. Data inconsistency and incompatibility contributed primarily to the failures. Although several of the six data sources by themselves could provide estimates at the national level, each had limitations. As a result of these findings, two cost-effective methodologios were proposed to estimate the number of commercial trucks operating in interstate commerce and their vehicle miles of travel by carrier type. Neither method required collecting additional data.

Research sponsored by U.S. Department of Energy.

* Center for Transportation Analysis, ORNL.

$\dagger_{\text {University of Tennessee, Knoxville, TN. }}$

${ }^{\dagger}$ Federal Highway Administration, U.S. Department of Transportation, Washington, DC.

\subsection{8}

\section{NATIONAL PROFILE ON COMMERCIALLY GENERATED LOW-LEVEL RADIOACTIVE MIXED WASTE}
J. A. Klein*
J. E. Mrochek*

R. L. Jolley*

I. W. Osborne-Lee*
A. A. Francis ${ }^{\dagger}$
T. Wright

(Abstract of ORNL-6731, December 1992)

This report details the findings and conclusions drawn from a survey undertaken as part of a joint U.S. Nuclear Regulatory Commission and U.S. Environmental Proteciton Agency-sponsored project entitled "National Profile on Commercially Generated Low-Level Radioactive Mixed Waste." The overall objective of the work was 
to compile a national profile on the volumes, characteristics, and treatability of commercially generated low-level mixed waste for 1990 by five major facility categories-academic, industrial, medical, and NRC-/Agreement State-licensed government facilities and nuclear utilities. Included in this report are descriptions of the methodology used to collect and collate the data, the procedures used to estimate the mixed waste generation rate for commercial facilities in the United States in 1990, and the identification of available treatment technologies to meet applicable EPA treatment standards (40 CFR Part 268) and, if possible, to render the hazardous component of specific mixed waste streams nonhazardous. The report also contains information on existing and potential commercial waste treatment facilities that may provide treatment for specific waste streams identified in the national survey. The report does not include any aspect of the Department of Energy's (DOE's) management of mixed waste and generally does not address wastes from remedial action activities.

Research sponsored by the Office of Nuclear Material Safety and Safeguards, U.S. Nuclear Regulatory Commission and by the Office of Solid Waste, U.S. Environmental Protection Agency.

* Chemical Technology Division.

$\dagger_{\text {Health and Safety Research Division. }}$
1.99

\section{A NOTE ON SAMPLING TO LOCATE RARE DEFECTIVES WITH STRONG PRIOR EVIDENCE}

\author{
T. Wright
}

[Abstract of Biometrika 79(4), 685 (1992)]

Inference about the number of defectives in a finite population is considered. Using a Bayesian model for computing the probability of unobserved defectives given the results of the sample, a criterion for sample size determination is introduced for two cases: (i) when there is a uniform prior, and (ii) when there is strong prior information. Depending on the value of the population size $N$, the savings in terms of a sampling effort rather than a census can be significant. When there is strong prior information, an explicit decision rule is given for determining whether a sample is needed or if the prior information alone is sufficient.

Research sponsored by Applied Mathematical Sciences Research Program, U.S. DOE Office of Energy Research.

\section{Applications of Statistical Methods}

\subsection{0}

\section{SELECTION OF FACTORS AFFECTING THE PRESENCE OF BROOKTROUT IN ADIRONDACK LAKES: A CASE STUDY}

\author{
J. J. Beauchamp S. W. Christensen* \\ E. P. Smith ${ }^{\dagger}$ \\ [Abstract of paper Can. J. Fish. Aquat. Sci. 49(3), 597 \\ (1992)]
}

We used multiple logistic regression techniques to develop models for estimating the probability of brook trout (Salvelinus fontinalis) presence/absence as a function of observable water chemistry variables and watershed characteristics. The data set consists of the Adirondack Lakes Survey Corporation data collected on 1469 lakes during 1984-87. Two models fitted to a randomly selected development subset of lakes, using two sets of candidate explanatory/predictor variables of particular interest, were compared on the basis of coefficient consistency and predictive ability. In addition to the usual maximum likelihood logistic regression results, we also applied collinearity and other associated diagnostics and variable-selection procedures designed specifically for the logistic regression model to arrive at parsimonious models. Both models correctly predicted fish presence in more then $85 \%$ of the model development set and more than $80 \%$ of the lakes in the verification data. For those variables appearing in both models, the 
signs of the estimated coefficients were the same and in agreement with expectation. The removal of influential observations, as indicated by the logistic regression diagnostics, caused all of the estimated coefficients to increase in absolute magnitude. This results in a model which is more sensitive to changes in the explanatory variables.

Research sponsored by the Adirondack Lakes Survey Corporation; by the U.S. Environmental Protection Agency; and by U.S. Department of Energy.

*Environmental Sciences Division.

†Virginia Polytechnic Institute and State University, Blacksburg, VA.

\subsection{1}

\section{MODELING SPEEDUP IN PARALLEL SPARSE MATRIX FACTORIZATION}
M. T. Heath
L. S. Ostrouchov*
G. Ostrouchov
C. H. Romine

[Abstract of Concurrency: Practice and Experience (in press)]

This paper is an attempt to explain the observed performance of sparse matrix factorization algorithms on parallel computers. In particular, we examine whether the disappointing performance of these algorithms is due to insuffcient parallelism in the problem or to the architectural characteristics of existing parallel computers. Through a series of theoretical models of increasing realism, we first determine upper and lower bounds on the speedup that can be expected in practice for this problem, and end with a parameterized model that is capable of reproducing the full range of behavior within these bounds, including the speedups actually observed in practice. This model suggests that the current limits on speedup in sparse factorization are due to poor communication performance of the present generation of parallel computer architectures rather than to a lack of parallelism in the problem.

Research sponsored by Applied Mathematical Sciences Research Program, U.S. DOE Office of Energy Research.

*University of Tennessee, Knoxville, TN.
1.102

\section{CONCENTRATION DEPENDENT \\ PROTECTION AGAINST X-RAY INDUCED CHROMOSOME ABERRATIONS IN HUMAN LYMPHOCYTES BY THE AMINOTHIOL WR-1065}

\author{
L. G. Littlefield* \\ S. P. Colyer ${ }^{*}$ \\ E. E. Joiner* \\ E. L. Frome \\ F. Sallam ${ }^{\dagger}$
}

[Abstract of Radiation Research (in press)]

WR-1065, the free-thiol form of WR-2721, protects against radiation-induced strand breaks, cell killing, transformation, hprt mutations, and chromosome aberrations in mammalian cells. Its radioprotective mechanisms are complex and may involve scavenging of $\mathrm{OH}$ radicals, chemical repair of DNA damage via hydrogen donation, or lowering of oxygen tension. We conducted studies to measure the efficiency of WR-1065 in modifying radiation-induced chromosome aberrations in human lymphocytes. $G_{0}$ lymphocytes were incubated for 30 min in medium containing 1-12 $\mathrm{mM} \mathrm{WR}-1065$, exposed to 0 or $3.1 \mathrm{~Gy} 220 \mathrm{kV} \mathrm{X}$ rays, washed, and cultured for evaluations of chromosome aberrations and micronuclei (MN). Neither proliferation kinetics nor baseline frequencies of aberrations or MN were affected in nonirradiated cultures incubated in WR-1065 for up to 45 min. Radiation-induced chromosome aberrations and $\mathrm{MN}$ varied inversely as a logarithmic function of thiol concentration and at extracellular concentrations of 8-12 mM, WR-1065 protected against $\geq 85 \%$ of $\mathrm{X}$-ray-induced chromosome damage as measured by either cytogenetic endpoint. Compared with our earlier findings, WR-1065 is more efficient in modulating $\mathrm{X}$-ray-induced chromosome aberrations than dimethyl sulfoxide, which is postulated to provide protection via selective scavenging of $\mathrm{OH}$ radicals. This observation suggests that mechanisms in addition to $\mathrm{OH}$ radical scavenging are involved in radioprotection afforded by this aminothiol.

Research sponsored by U.S. DOE/ORAU contract and NCI grant.

*Oak Ridge Institute of Science and Education, Oak Ridge, TN.

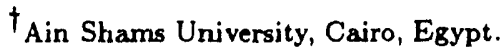


1.103

\section{EXPERIENCE WITH THE 'CYTOCHALASIN-B METHOD' FOR QUANTIFYING RADIATION- INDUCED MICRONUCLEI IN HUMAN LYMPHOCYTES}

\author{
L. G. Littlefield * \\ F. Sallam ${ }^{\dagger}$ \\ A. M. Sayer* \\ R. A. Kleinerman ${ }^{\ddagger}$
}

[Abstract of paper presented at the 9th International Congress on Radiation Research, Toronto, Canada, July 712, 1991; Proc. Radiation Research: A Twentieth-Century Perspective, Vol. II: Congress Proceedings, pp. 183-188, Academic Press (1992)]

Asymmetrical chromosome aberrations in lymphocyte metaphases are sensitive biomarkers of radiation dose in recently exposed persons. A portion of these induced acentric fragments will be excluded from the main nucleus during postmitotic nuclear reformation, and appear as micronuclei (MN) at the second interphase postexposure. Scoring of MN has been recommended as a simple alternative for quantifying chromosome damage, since the method can be accomplished rapidly and requires less expertise than that needed for aberration analysis. In 1985, Fenech and Morley proposed an innovative approach for evaluating radiation induced $\mathrm{MN}$ in lymphocytes. The method employs cytochalasin-B (Cyt-B) to inhibit cytokinesis in proliferating lymphocytes, yielding binucleate products that contain two discrete daughter nuclei within a single cytoplasmic membrane. Numerous laboratories have now employed this method to obtain interphase II cells for evaluations of baseline and induced MN. As experience using the Cyt-B method has been gained, it is now apparent that several variables may affect interpretation of data. For example, because MN arise not only from acentrics, but also from lagging whole chromosomes, background $\mathrm{MN}$ frequencies tend to be quite variable, which could possibly confound use of MN to estimate dose in persons having suspected low dose exposures. Several technical variables may also affect recovery of $\mathrm{MN}$ in irradiated lumphocytes. We have used the Cyt-B method to compare $\mathrm{MN}$ and aberration frequencies in several persons having real or suspected radiation exposures, and have also used the technique to compare effectiveness of radiations having differing LETs. An overview of our findings will be presented to highlight applications ind limitations of the MN system in quantifying radiation induced chromosome damage in human lymphocytes.

Research sponsored by U.S. DOE/ORAU contract and NCI-DOE Interagency Agreement.

*Oak Ridge Associated Universitie, Oak Ridge, TN.

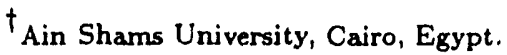

$\ddagger_{\text {National Cancer institute, Bethesda, MD. }}$

1.104

\section{RELATIONSHIP BETWEEN TRUCK ACCIDENTS AND HIGHWAY GEOMETRIC DESIGN: A POISSON REGRESSION APPROACH}

\author{
S.-P. Miaou* P. S. Hu* \\ T. Wright A. K. Rathi* \\ S. C. Davis*
}

[Abstract of Transportation Research Record 1376, 10 (1992)]

A Poisson regression model is proposed to establish empirical relationships between truck accidents and key highway geometric design variables. For a particular road section, the number of trucks involved in accidents over 1 year was assumed to be Poisson-distributed. The Poisson rate was related to the road section's geometric, traffic, and other explanatory variables (or covariates) by a loglinear function, which ensures that the rate is always nonnegative. The primary data source used was the Highway Safety Information System (HSIS), administered by FHWA. Highway geometric and traffic data for rural Interstate highways and the associated truck accidents in one HSIS state from 1985 to 1987 were used to illustrate the proposed model. The maximum likelihood method was used to estimate the model coefficients. The final model suggested that annual average daily traffic per lane, horizontal curvature, and vertical grade were significantly correlated with truck accident involvement rate but that shoulder width had comparably less correlation. Goodness-of-fit test statistics indicated that extra variation (or overdispersion) existed in the developed Poisson model, which was most likely due to the uncertainties in truck exposure data and omitted variables in the model. This suggests that better quality in truck exposure data and additional covariates could probably improve the current model. Subsequent analyses subsiested, 
however, that this overdispersion did not change that conclusions about the relationships between truck accidents and the examined geometric and traffic variables.

Research sponsored by U.S. DOE and Office of Safety and Traffic Operations Research and Development, FHWA, U.S. Department of Transportation.

*Energy' Division.

1.105

\section{MARROW CELL KINETICS MODEL: EQUTVALENT PROMPT DOSE APPROXIMATIONS FOR TWO SPECIAL CASES}

\author{
M. D. Morris T. D. Jones*
}

(Abstract of ORNL/TM-12255, November 1992)

Two simple algebraic expressions are described for approximating the "equivalent prompt dose" as defined in the model of Jones et al. These approximations apply to two specific radiation exposure patterns: (1) a pulsed dose immediately followed by a protracted exposure at relatively low, constant dose rate and (2) an exponentially decreasing exposure field.

Research sponsored by U.S. Department of Energy and Defense Nuclear Agency.

${ }^{*}$ Health and Safety Research Division.

1.106

\section{A CELL KINETICS MODEL OF RADIATION - INDUCED MYELOPOIESIS: RATE COEFFICIENT ESTIMATES FOR MOUSE, RAT, SHEEP, SWINE, DOG, AND BURRO IRRADIATED BY PHOTONS}

$$
\begin{aligned}
& \text { M. D. Morris T. D. Jones* } \\
& \text { R. W. Young } \\
& \text { [Abstract of Radiation Research (in press)] }
\end{aligned}
$$

Rate coefficients in the model of cell kinetics and mortality introduced by Jones et al. [Radiat. Res. 128, 258-266 (1991)] are estimated using mortality data from 27 animal experiments. Adjustments are made for the six species and three nominal classes of gamma radiation represented in these studies. The evaluated model fits most of the mortality data quite well when the rate coefficient representating cellular proliferation is fitted to in. dividual species and each of the other coefficients are given a single value across the entire data set. Results are qualitatively similar to those reported by Morris et al. [Radiat. Res. 128, 267-275 (1991)] who estimated the rate coefficients from a limited number of mouse studies involving only $250 \mathrm{kVp} \mathrm{x}$-rays. As in the earlier study, estimates here lead to greater cell survival than is observed for nonterminally differentiated reproducing cells from the marrow.

Research sponsored by the Defense Nuclear Agency and U.S. Department of Energy.

*Health and Safety Research Division.

†Defense Nuclear Agency, Washington, DC.

1.107

COMPUTER COMMUNICATION: WHAT'S YOUR E-MAIL ADDRESS?

\section{G. Ostrouchov}

[Abstract of Statistical Computing and Statistical Graphics Newsletter 2(2), 22 (1991)]

This article discusses electronic mail on the Internet and its use in communicating with other researchers. The domain name address system and routing of messages are explained, and several sources of electronic mail addresses of researchers in mathematical sciences are included.

Research sponsored by U.S. Department of Energy.

\subsection{8 \\ COMPUTER COMMUNICATION: RESOURCE DISCOVERY}

\section{G. Ostrouchov} [Abstract of Statistical Computing and Statistical Graphics
Newsletter 3(1), 21 (1992)]

This article discusses several resources available over the Internet. This includes archie and rarchic for finding resources on the Internet, a new document describing the Internet, and PVM (Parallel Virtual Machine) for harnessing parallel power from a heterogeneous collection of networked computers.

Research sponsored by Applied Mathematical Sciences Research Program, U.S. DOE Office of Energy Research. 
1.109

\section{COMPUTER COMMUNICATION: ALTERNATE CONNECTIONS AND WHITE PAGES}

\section{G. Ostrouchov}

[Abstract of Statistical Computing and Statistical Graphics Newsletter 3(2), 22 (1992)]

Many researchers in statistics do not have 2 direct link to the Internet and thus cannot take advantage of the many resources it offers. Some alternatives to a direct Internet link are discussed. These alternatives allow indirect Internet access. Also, several "white pages" services on the Internet are discussed.

Research sponsored by U.S. Department of Energy.

\subsection{0}

\section{USING WEIBULL STATISTICS TO ANALYZE ION BEAM ENHANCED ADHESION AS MEASURED BY THE PULL TEST}
J. E. Pawel ${ }^{*}$
D. J. Downing
L. J. Romana*
W. E. Lever
C. J. McHargue ${ }^{\dagger}$
J. J. Wert ${ }^{\ddagger}$

[Abstract of paper presented at the Materials Research Society 1991 Fall Meeting, Boston, MA, December 2-6, 1991; Proc. Mat. Res. Soc. Symp. Vol. 239, p. 541 (1992)]

The adhesion of iron films to single crystal $\mathrm{Al}_{2} \mathrm{O}_{3}$ substrates was investigated using a pull test. Chromium $(300 \mathrm{keV})$ or nickel $(340 \mathrm{keV})$ ions were implanted to a fluence of $1 \times 10^{15}$ ions$\mathrm{cm}^{-2}$ after film deposition. The adhesion test results were widely scattered due to a random distribution of interfacial flaw sizes controlling the failure nucleation. Because Weibull statistics were developed to describe the failure probability due to a population of flaw-initiated cracks, the Weibull distribution was chosen to analyze the data. Modifications in the adhesion strength due to the ion implantation were reflected in the failure distributions. It was found that the chromium ions improved the adhesion of the $\mathrm{Fe} / \mathrm{Al}_{2} \mathrm{O}_{3}$ system while the implantation of nickel did not.

\footnotetext{
Research sponsored by U.S. DOE, Conservation and Renewable Energs:

* Metals and Ceramics Division.

$\dagger_{\text {University of Tennessee, Knoxville, TN. }}$

†Vanderbilt University, Nashville, TN.
}

1.111

JOB FACTORS, RADIATION

AND CANCER MORTALITY AT

OAK RIDGE NATIONAL

LABORATORY: FOLLOW-UP THROUGH 1984
S. Wing ${ }^{*}$
C. M. Shy ${ }^{*}$
J. L. Wood ${ }^{*}$
S. Wolf*
D. L. Cragle ${ }^{\dagger}$
W. Tankersley ${ }^{\dagger}$

E. L. Frome

[Abstract of American Journal of Industrial Medicine (in press)]

A previous study of mortality among white men hired at Oak Ridge National Laboratory between 1943 and $1972(n=8,318)$ revealed an association between low dose external penetrating ionizing radiation and cancer mortality in follow-up through 1984. The association was not observed in follow-up through 1977. This report considers the role of possible selection and confounding factors not previously studied. Control for hire during the World War II era and employment duration of less than one year had little effect on the radiation risk estimates. Risks associated with length of time spent in 15 job categories were considered as proxies for the effects of other occupational carcinogens. Adjustment for employment duration in each job category one at a time produced only small changes in the radiation risk estimate. Adjustment for potential exposures to beryllium, lead and mercury also had little effect on the radiation risk estimates. These analyses suggest that selection factors and potential for chemical exposure do not account for the previously noted association of external radiation dose with cancer mortality. However, power to detect effects of chemical exposures is limited by a lack of individual exposure measures.

Research sponsored by U.S. DOEjORAU cuntract.

*University of North Carolina, Chapel Hill, NC.

†Oak Ridge Associated Universities, Oak Ridge, TN. 
1.112

\section{ASSESSMENT OF EN ROUTE SECTOR AND TERMINAL AIR TRAFFIC CONTROL PERFORMANCE}

T. A. Vineyard*

R. C. Durfee ${ }^{\dagger}$

D. M. Flanagan

R. T. Goeltz \#

J. A. Rome**

J. L. Smyre ${ }^{\dagger}$

D. J. Downing

J. J. Edwards ${ }^{\ddagger}$

M. C. Fletcher $\S$

G. Ostrouchov

M. J. Saltmarsh***

W. R. Wing***

(Abstract of K/DSRD-733, June 1991)

In March 1991, the Federal Aviation Administration (FAA) requested (through an interagency agreernent with the Department of Energy) assistance from Martin Marietta Energy Systems, Inc. (Energy Systems), in a comprehensive study designed to assess en route sector and terminal Air Traffic Control performance. The study was in response to a Congressional mandate to evaluate system performance and develop recommendations and options to increase and improve en route sector throughput.

Research sponsored by U.S. Department of Energy.

*Data Systems R\&D.

${ }^{\dagger}$ Computing Applications Division.

$\ddagger_{\text {Business Systems. }}$

$\S_{\text {Computing and Telecommunications Division. }}$

\# Energy Division.

**Fusion Energy Division.

*** Central Management. 
Section 2

NUCLEAR DATA MEASUREMENT AND EVALUATION 


\title{
2.0. INTRODUCTION
}

\author{
D. C. Larson
}

Activities in the Nuclear Data Measurement and Evaluation Section center around measurements of neutron cross sections and related quantities performed using the Oak Ridge Electron Linear Accelerator (ORELA). Both basic research and work of interest to applications of nuclear physics are performed. In addition to measurement work, complete neutron cross section evaluations are produced, and model codes which support the evaluation work are developed and improved.

We had several significant achievements during this time period, which will be highlighted in the following paragraphs. First, at 8:48 p.m. on October 13, 1992, ORELA passed the 100,000 hours of operation milestone. It became operational in October, 1969 and during 23 years of operation has averaged nearly 4400 hours of operation per year. The availability of ORELA has been over $90 \%$ for the past 20 years. The Operations Staff, consisting of two engineers and three operators, still keeps us operating five days per week around the clock. Perhaps as notable as the fact of 100,000 operating hours is the record keeping over the 23 years which provided this information. The machine still regularly exceeds the acceptance criteria when required for an experiment. As a result of this achievement, the ORELA Operations Team won an ORNL World-Class Teamwork Award.

Since its inception, over 500 publications and several hundred reports have been written to describe ORELA-based research. Capture cross sections for over 180 nuclides have been measured to meet applied needs and for astrophysics studies, and total, fission, elastic and inelastic cross sections, as well as neutron and gammaray production cross sections, have been measured. ORELA data have impacted over $80 \%$ of the evaluations in the U. S. Evaluated Nuclear Data File (ENDF/B), used worldwide for nuclear technology development. It has been, and continues to be a very productive facility.

One measure of the quality of ORELA-based research is the fact that in 1992 two ORELA staff members, Jack Harvey and Nat Hill, were on teams which won two of the ten Martin Marietta Energy Systems Technical Achievement Awards presented to ORNL. A team led by Jack Harvey with members Nat Hill, and two researchers from the Technical University of Vienna received an award for a measurement done at ORELA which provided the first meaningful value of the induced electric polarizability of the neutron. Over twenty searches at other laboratories by various methods had failed to verify an electric polarizability for the neutron. Current understanding of the structure of the neutron is based on the quark model, which implies a non-zero induced polarizability. Results of the difficult but successful transmission measurements give a new test to be met by quark model calculations.

Harvey and Hill were also members of a team led by Herb Mook of the Solid State Division investigating lattice dynamics of high-temperature (high-Tc) superconductors at ORELA. Very accurate neutron transmission measurements were used to demonstrate that phonon states in the high-Tc material must soften at the superconducting transition. This result is important because it shows for the first time that lattice vibrations and high-Tc superconductivity are closely related. This work is an outstanding example of the interdisciplinary interaction that is possible 
at national laboratory. In both cases, ORELA was selected for its intensity and low background properties.

Cross sections for $\mathrm{Pu}-239$ play an important role in efficient and safe operation of nuclear reactors. In the resolved resonance region of the $\mathrm{Pu}-239$ fission cross section there has been a discrepancy in the measured average value over wide neutron energy intervals as well as a discrepancy in the value of the cross sections between resonances. This was one of six major problems in nuclear data initially singled out for study by a Nuclear Energy Agency international committee. Very careful additional measurements at ORELA by L. W. Weston and J. H. Todd, and corresponding resonance parameter analysis by $H$. Derrien (Japan) have been carried out and this important problem is now considered solved. Publication of the results are underway and the international committee has given preliminary approval of the results. This is an example of how nuclear data measurements at ORELA, with international cooperation, continue to eliminate important discrepancies in the data base for applied work.

ORELA staff member J. K. Dickens has been selected as General Chairman of the 1994 International Conference on Nuclear Data for Science and Technology, to be held in Gatlinburg. This is the next in a series of conferences previously held in Knoxville (1979), Antwerp (1982), Santa Fe (1985), Mito (1988) and Julich (1991). The program will include a wide range of interdisciplinary studies dealing with measurement, calculation, evaluation, application and use of nuclear data. Both energy and non-energy related data will be treated with emphasis on application-oriented numerical data. Special attention will be given to new applications (technology transfer) of nuclear data. The conference has national and international sponsorship, and traditionally gathers about 300 experts in the nuclear data field.

Our Section hosted an international Specialists' Meeting on Evaluation and Processing of Covariance Data in October, 1992. Over half of the 21 participants were non-US. This Nuclear Energy Agency sponsored meeting was approved to address all problems concerning uncertainty estimates of evaluated nuclear data. The papers focussed on reviewing the need for evaluated uncertainty and covariance information in various fields of applied nuclear science, methods developed for deriving uncertainty estimates from existing data and theoretical considerations, representation of information in covariance files, and problems processing the data.

Several members of our Section are also involved in a NEA Working Group on International Evaluation Cooperation. The purpose of this activity is to identify and eliminate cross section discrepancies among the four major evaluated data libraries in the world. As the work continues, the libraries are expected to converge into one library for world-wide use.

Nuclear model and analysis codes continue to be improved and be used around the world. The resonance parameter code SAMMY developed in our Section by N. M. Larson (CAD) can now analyze differential elastic scattering data, including corrections for finite size samples, and is nearly ready to analyze capture cross section data, again correcting the data for finite size and multiple scattering effects. These developments will keep it unique and at the front of resonance parameter analysis code development. The nuclear model code TNG, developed by C. Y. $\mathrm{Fu}$, continues to broaden its capabilities and improve its physics content through improvements to the level density. It is the workhorse of cross section evaluation work for the $\mathrm{MeV}$ region in our Section. 
Unfortunately, even the above achievements are not sufficient to retain adequate funding. In two years, our total budget has been cut a factor of five from $\$ 3.8 \mathrm{M}$ (FY92) to $\$ 0.75 \mathrm{M}$ (FY94). This cut reflects a combination of budget pressures in DOE/Nuclear Physics and loss of appreciation of the importance of nuclear data to technology development. In 1991 there were three major producers of nuclear data in the US, now two are gone (at ANL and LANL), and we are severely hampered. ORELA is the last intense, pulsed neutron source in the US able to provide high resolution neutron data in the resonance region. The situation around the world is not much better, and international agencies have become very concerned about the loss of nuclear data measurement capability. It soon will be extinct if present policies are not reversed. We are actively working to identify our customer base and provide products and services of value to them, thus broadening our base of support to both new and traditional users of nuclear data. 


\section{EXPERIMENTAL RESULTS AND THEIR ANALYSIS}

2.1

\section{EXPERIMENTAL AND CALCULATED EXCITATION FUNCTIONS FOR DISCRETE-LINE GAMMA-RAY PRODUCTION DUE TO 1-40 MeV NEUTRON INTERACTIONS WITH ${ }^{56} \mathrm{Fe}$}

\author{
J. K. Dickens \\ C. Y. Fu
}

D. M. Hetrick* J. H. Todd ${ }^{\dagger}$

[Abstract of paper presented at the International Conference on Nuclear Dala for Science and Technology, Julich, Federal Republic of Germany, May 13-17, 1991; Proc. pp. 307-309, S. M. Qaim, Ed., Springer-Verlag (1992)]

Measuring cross sections for gamma-ray production from tertiary reactions is one of the ways to gain experimental information about these reactions. To this end, inelastic and other nonelastic neutron iteractions with ${ }^{56} \mathrm{Fe}$ have been studied for incident neutron energies between 0.8 and $41 \mathrm{MeV}$. An iron sample isotopically enriched in the mass 56 isotope was used. Gamma rays representing 70 transitions among levels in residual nuclei were identified, and production cross sectiors were deduced. The reactions studied were ${ }^{56} \mathrm{Fe}\left(n, n^{\prime}\right){ }^{56} \mathrm{Fe},{ }^{56} \mathrm{Fe}(n, p){ }^{56} \mathrm{Mn},{ }^{56} \mathrm{Fe}(n, 2 n){ }^{55} \mathrm{Fe}$, ${ }^{56} \mathrm{Fe}(n, d+n, n p){ }^{55} \mathrm{Mn},{ }^{56} \mathrm{Fe}(n, t+n, n d+n, 2 n p)$ ${ }^{54} \mathrm{Mn}, \quad{ }^{56} \mathrm{Fe}(n, \alpha){ }^{53} \mathrm{Cr},{ }^{56} \mathrm{Fe}(n, n \alpha){ }^{52} \mathrm{Cr}$, and ${ }^{56} \mathrm{Fe}(n, 3 n){ }^{54} \mathrm{Fe}$. Experimental excitation functions have been compared with cross sections calculated using the nuclear reaction model code TNG, with generally favorable results.

Research sponsored by U.S. DOE Office of Energy Research, Division of Nuclear Physics.

* Computing and Telecommunications Division.

$\dagger_{\text {Instrumentation and Controls Division. }}$
2.2

MEASUREMENTS AND CALCULATIONS OF RADIONUCLIDES CREATED BY

${ }^{181} \mathrm{Ta}(\gamma, x$ nyp $)$ REACTIONS FOR

BREMSSTRAHLUNG PHOTONS PRODUCED BY 150-MeV ELECTRONS

\section{J. K. Dickens M. A. Miller*}

[Abstract of Nucl. Sci. Eng. (in press)]

Twenty-three radionuclides, including isomers, from ${ }^{168 \mathrm{rn}} \mathrm{Lu}$ to ${ }^{180} \mathrm{Ta}$, having half lives between $7 \mathrm{~min}$ and $1.4 \mathrm{yr}$, were produced by photon interactions with a sample of elemental tantalum. Yields of production were deduced from decay gamma-ray data obtained using highresolution detection systems and range over six orders of magnitude. The measured yield data for masses $\geq 171$ were compared with calculated values. Good agreement was obtained for masses $\geq 174$, but for lighter masses the calculations tend to overestimate the experimental yields by up to a factor of eight. These results should have practical use not only for present electron accelerator operation but also for shielding calculations needed for future accelerator designs and applications.

Research sponsored by U.S. DOE Division of Nuclear Physics.

*University of Wisconsin, River Falls, WI.

\section{3}

MEASUREMENTS OF ${ }^{181} \mathrm{Ta}(\gamma, x n y p)$ REACTIONS FOR BREMSSTRAHLUNG PHOTONS PRODUCED BY 150-MeV ELECTRONS

\section{J. K. Dickens M. A. Niller*}

[Abstract of paper presented at the Southeastern Section Meeting of the American Physical Society, Oak Ridge, TN, November 12-14, 1992; Proc. Vol. 37(7), p. 1677 (1992)]

Twenty-three radionuclides, including isomers, from ${ }^{168 \pi \mathrm{m}} \mathrm{Lu}$ to ${ }^{180 \mathrm{~m}} \mathrm{Ta}$, having half lives between $7 \mathrm{~min}$ and $1.4 \mathrm{yr}$ were produced by photon interactions with elemental tantalum. Yields of production, ranging over six orders in magni- 
tude, were deduced from gamma-ray decay data obtained using high-resolution Ge detectors. The measured yield data decrease by approximately a factor of two for each emitted nucleon. Calculated yields based on Levinger's model for photon interactions in nuclei followed by intranuclear cascade and then followed by compound nucleus decay were compared with yield data for final masses $\geq 171$ with generally good agreement.

Research sponsored by U.S. DOE Division of Nuclear Physics.

*University of Wisconsin, River Falls, WI.

\section{4}

\section{MEASUREMENT OF THE NITROGEN TOTAL CROSS SECTION FROM $0.5 \mathrm{eV}$ TO $50 \mathrm{MeV}$ AND ANALYSIS OF THE 433-keV RESONANCE}

\section{J. A. Harvey N. W. Hill* N. M. Larson ${ }^{\dagger}$}

[Abstract of paper presented at the International Conference on Nuclear Data for Science and Technology, Julich, Federal Republic of Germany, May 13-17, 1991; Proc. pp. 729-731, S. M. Qaim, Ed., Springer-Verlag (1992)]

High-resolution neutron transmission measurements have been made on several thicknesses of nitrogen gas samples from $0.5 \mathrm{eV}$ to $50 \mathrm{MeV}$ at the Oak Ridge Electron Linear Accelerator (ORELA). A preliminary $\mathrm{R}$-matrix analysis has been done for resonances up to $800 \mathrm{keV}$. An $\mathrm{R}$ matrix analysis of previous data was done by LANL for ENDF/B-VI, including the lowest energy resonance in ${ }^{14} \mathrm{~N}$ at $433 \mathrm{keV}$. They found a spin of $3 / 2$ (with $\ell=1$ ) and a peak cross section of $7.0 \mathrm{~b}$. Analysis of the present data yield a spin of $7 / 2$ (requiring $\ell \leq 2$ ) and a peak cross section of $11.5 \mathrm{~b}$ for this resonance. These results are important for transport calculations of neutrons through air. Scattering measurements are planned to determined the parity of this resonance.

Research sponsored by U.S. DOE Office of Energy Research, Division of Nuclear Physics.

*Instrumentation and Controls Division.

${ }^{\dagger}$ Computing and Telecornmunications Division.
2.5

\section{NEUTRON PHYSICS AT THE OAK RIDGE NATIONAL LABORATORY}

\section{J. A. Harvey}

[Abstract of paper presented at the Southeastern Section Meeting of the American Physical Society, Oak Ridge, TN, November 12-14, 1992; Proc. Vol. 37(7), p. 1641 (1992)]

Neutron physics began at ORNL soon after the operation of the Graphite Reactor in November 1943 emphasizing both basic physics (nuclear and solid state) and applied physics to satisfy nuclear data needs for reactor development. Notable achievements in the 1940 s were the first measurement of the decay of the neutron (and its half life) by Art Snell and coworkers, and the first Laue diffraction from powdered crystals using thermal neutrons by Ernie Wollan and Cliff Shull. After the installation of a $2 \mathrm{MV}$ and a $5 \mathrm{MV}$ Van de Graaff accelerator in the new High Voltage Laboratory in the early 1950s, aggressive fast neutron programs were initiated by Joe Fowler to measure fission, capture, scattering and total neutron cross sections for nuclides throughout the periodic table. With the operation of the Oak Ridge Research Reactor (ORR) in the late 1950s, thermal and resonance neutron beams were used to study details of the fission process, to measure the neutron total and capture cross sections of many isotopes (both stable and radioactive) from thermal to about $10 \mathrm{keV}$ neutron energy using microsecond pulses from a fast chopper and flight path up to 180 meters long, and for neutron diffraction studies on magnetic crystals. Other experiments at the ORR were measurements of the gravitational acceleration of free neutrons, of the static electric dipole moment of the neutron, and of the electron neutrino angular correlation from $\mathrm{He}-6$. To meet demands for improved energy resolution and intensity for measurements needed in the $\mathrm{keV}$ energy region for the proposed fast breeder reactor and for basic physics, the Oak Ridge Electron Linear Accelerator (ORELA) laboratory was constructed in the late 1960s. ORELA has been in operation for over 20 years producing high intensity nanosecond pulses for neutron measurements from $\sim 10^{-3} \mathrm{eV}$ to $\sim 100 \mathrm{MeV}$. Almost all types of cross measurements have been made at ORELA such as fission (to study subthreshold fission of the even-A heavy nuclides and of the fissile nuclides 
of importance to reactor designers), capture (to study the formation of heavy elements in stars by neutron capture of $\mathrm{keV}$ energy neutrons), and total and scattering (to study refinements of nuclear reaction models). Recent experiments at ORELA have discovered phonon softening around the critical temperature of high temperature superconductors and produced the first significant measurement of the induced electric polarizability of the neutron.

Research sponsored by U.S. DOE Division of Nuclear Physics.

2.6

\section{REVIEW OF FISSION PRODUCT \\ CAPTURE MEASUREMENTS AT THE OAK RIDGE ELECTRON LINEAR ACCELERATOR (ORELA)}

\section{C. Larson $\quad$ R. L. Macklin}

[Abstract of paper presented at the NEANSC Specialists' Meeting on Fission Product Nuclear Data, JAERJ Tokai Research Establishment, Ibaraki-Ken, Japan, May 28-29, 1992; Proc. pp. 127-138 (1992)]

The 40-meter capture system of $R$. L. Macklin at ORELA has been used to measure capture cross sections for over eighty isotopes between $A$ $=75$ and 161 , the primary mass region of interest for fission products. This review outlines the primary features of the capture system, lists the isotopes measured and their references, and describes recent changes incorporated in the capture system.

Research sponsored by U.S. DOE Office of Energy Rescarch, Division of Nuclear Physics.

\section{7}

\section{WHITE SOURCE GAMMA-RAY- PRODUCTION SPECTRAL MEASUREMENT FACILITIES IN THE U.S.}
D. C. Larson
J. K. Dickens
R. O. Nelson*
S. A. Wender*

A bstract of paper presented at the International Conference on Nuclear Data for Science and Technology, Julich, Federal Republic of Germany, May 13-17, 1991; Proc. pp. 422-426, S. M. Qaim, Ed., Springer-Verlag (1992)]

The two primary neutron sources for measuring gamma-ray-production (GRP) cross sections for basic and applied work in the U.S.A. are the Oak Ridge Electron Linear Accelerator (ORELA) located at Oak Ridge National Laboratory (ORNL) and the Weapons Nuclear Research (WNR) Facility located at the Los Alamos National Laboratory (LANL). ORELA is based on a $180-\mathrm{MeV}$ electron linear accelerator, while the WNR facility uses the Los Alamos Meson Physics Facility $800 \mathrm{MeV}$ proton beam to produce neutrons. The facilities collectively cover the neutronenergy range from thermal to over $700 \mathrm{MeV}$. The paper describes the present capabilities for GRP measurements at each facility.

Research sponsored by U.S. Department of Energy.

* Los Alamos National L.sboratory, Los Alamos, NM.

\section{8}

\section{STUDY OF RADIONUCLIDES CREATED BY ${ }^{181} \mathrm{Ta}(\gamma$, xnyp $)$ REACTIONS FOR BREMSSTRAHLUNG PHOTONS PRODUCED BY 150-MeV ELECTRONS}
M. A. Miller*
J. K. Dickens

(Abstract of ORNL/TM-11944, December 1991)

Ten radionuclides, including isomers, from ${ }^{172} \mathrm{Ta}$ to ${ }^{180} \mathrm{Ta}$ and ${ }^{180 m} \mathrm{Hf}$ were produced by photon interactions with a sample of elemental tantalum and measured by counting photons using a high-resolution detection system. Relative yields of these radionuclides were obtained. In addition, precision half lives were obtained for $175,176,180 \mathrm{Ta}$ and ${ }^{180 \mathrm{~m}} \mathrm{Hf}$. Those obtained for the three $\mathrm{Ta}$ isotopes agree with previously reported values. For ${ }^{180 m} \mathrm{Hf}$, the present measurements resulted in a half life determination of 6.05 $\pm 0.06 \mathrm{hr}$, or about $10 \%$ longer than the currently adopted value for this half life.

Research sponsored by U.S. DOE Division of Nuclear Physics.

*University of Wisconsin, River Falls, WI. 
2.9

\section{PROGRESS AND PROBLEMS IN ENERGY-RELATED NUCLEAR DATA}

\author{
R. W. Peelle
}

(Abstract of paper presented at the International Conference on Nuclear Data for Science and Technology, Julich, Germany, May 13-17, 1991; Proc. pp. 1011-1013, S. M. Qaim, Ed., Springer-Verlag (1992)]

The International Conference is reviewed relative to needs for measured data in nuclear energy applications. Notable achievernents were recorded in several research areas. Elsewhere, obstacles have inhibited development of adequate data. Major data areas are identified with various levels of progress during recent years. The present energyrelated nuclear data base is characterized as inadequate relative to a rising standard of quality. Ongoing efforts can achieve the continual improvement required for future technology.

Research sponsored by U.S. DOE Division of Nuclear Physics.

2.10

\section{${ }^{88} \mathrm{Ni}+n$ TRANSMISSION, DIFFERENTIAL ELASTIC SCATTERING AND CAPTURE MEASUREMENTS AND ANALYSIS UP TO $813 \mathrm{keV}$}

\author{
C. M. Perey \\ J. A. Harvey \\ F. G. Perey \\ N. M. Larson ${ }^{\dagger}$ \\ D. C. Larson \\ N. W. Hill* \\ [Abstract of Phys. Rev. C (in press)]
}

High-resolution neutron cross section measurements for ${ }^{58} \mathrm{Ni}$-enriched targets were made at the Oak Ridge Electron Linear Accelerator of transmission below $20 \mathrm{MeV}$, of differential elastic scattering from $10 \mathrm{keV}$ to $5 \mathrm{MeV}$, and of capture from $2.6 \mathrm{keV}$ to $2.5 \mathrm{MeV}$. The transmission data were analyzed from 0.1 to $813 \mathrm{keV}$ with a multilevel R-matrix code which uses Bayes' theorem for the fitting process. This code provides energies and neutron widths of resonances within the analyzed region, as well as a possible parameterization for resonances external to that region, as a way of describing the smooth cross section over the entire energy range. The differential elastic data at different scattering angles were compared to theoretical calculations from 30 to 813 $\mathrm{keV}$ using an R-function code which is based on the Blatt-Biedenharn formalism. Various combinations of spin and parity were tested to predict cross sections for the well-defined $\ell \geq 0$ resonances, and comparison with the data then provided spin and parity assignments for most of these resonances. The capture data were analyzed from 5 to $450 \mathrm{keV}$ with a least-squares fitting code using the Breit-Wigner formula. The resulting set of resonance parameters yields values for the thermal total and capture cross sections within experimental uncertainties. A total of 482 resonances are reported, of which five are fictitious $s$-wave resonances outside the analyzed energy region and 61 are seen and analyzed only in the capture data. The reduced widths of the $61 \mathrm{~s}$-wave resonances follow the Porter-Thomas distribution and their nearest neighbor spacings agree with the Wigner distribution. The average s-wave level spacing is $13.1 \pm 0.9 \mathrm{keV}$ and the s-wave strength function is $(3.2 \pm 0.6) \times 10^{-4}$. Since most of the large non s-wave resonances have their angular momentum assigned with confidence, the strengih functions for the $p$ - and $d$-wave resonances could be determined; values are $(1.3 \pm 0.2) \times 10^{-4}$ and $(3.0 \pm 0.4) \times 10^{-4}$, respectively. The level densities calculated with the Fermi-gas model for $\ell=0$ and $\ell \geq 0$ resonances are compared with the cumulative number of observed resonances. The average radiation widths were deduced from resonances analyzed in the three data sets below $450 \mathrm{keV}$. The mean values and standard deviations of the distributions of the radiation widths are $2.3 \pm 1.7$ $\mathrm{eV}$ for the s-wave resonances, $0.77 \pm 0.32 \mathrm{eV}$ for the p-wave resonances, and $1.4 \pm 0.5 \mathrm{eV}$ for the $\mathrm{d}$-wave resonances. The average capture cross section as a function of the incident neutron energy is compared to a prediction based on the tail of the giant electric dipole resonance.

Research sponsored by U.S. DOE Division of Nuclear Physics.

\footnotetext{
*Instrumentation and Controls Division.

${ }^{\dagger}$ Computing and Telecommunications Division.
} 
2.11

\section{${ }^{56} \mathrm{Fe}$ AND ${ }^{60} \mathrm{Ni}$ RESONANCE PARAMETERS}

\author{
C. M. Perey F. G. Perey \\ J. A. Harvey N. W. Hill ${ }^{*}$ \\ N. M. Larson ${ }^{\dagger}$
}

[Abstract of paper presented at the International Conference on Nuclear Data for Science and Technology, Julich, Federal Republic of Germany, May 13-17, 1991; Proc. pp. 41-43, S. M. Qaim, Ed., Springer-Verlag (1992)]

High-resolution neutron transmission and differential elastic-scattering measurements were made for a ${ }^{56} \mathrm{Fe}$-enriched iron target at the Oak Ridge Electron Linear Accelerator (ORELA). A natural iron target was used for transmission measurements below $160 \mathrm{keV}$. The data were analyzed from 5 to $850 \mathrm{keV}$. Parameters were obtained for $33 \ell=0$ and $242 \ell>0$ resonances. New ${ }^{6}$ Li-glass transmission data were acquired for two ${ }^{60} \mathrm{Ni}$ enriched sample thicknesses. The neutron width for the $2.253-\mathrm{keV}$ resonance was determined to be $59.3 \pm 0.6 \mathrm{meV}$ and the radiation width $553 \pm 50$ $\mathrm{meV}$.

Research sponsored by U.S. DOE Office of Energy Research, Division of Nuclear Physics.

*Instrumentation and Controls Division..

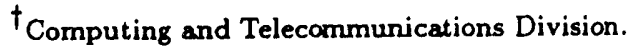

\subsection{2}

\section{ELECTRIC PROPERTIES OF THE NEUTRON FROM PRECISION CROSS SECTION MEASUREMENTS}
J. Schmiedmayer*
P. Riehs*
J. A. Harvey
N. W. Hill

[Abstract of paper presented at the International Conference on Nuclear Data for Science and Technology, Julich, Federal Republic of Germany, May 13-17, 1991; Proc. pp. 163-165, S. M. Qaim, Ed., Springer-Verlag (1992); also Spring Meeting of Nuclear Physics Sections, University of Salzburg, Austria, February 24-28, 1992]

In neutron nuclear interaction the electric charge structure of the neutron (first inferred by its high magnetic moment and now established by the quark picture for hadrons) interacts with the Coulomb field of atomic nuclei. Contributions are expected from the electric polarizability $\alpha_{n}$, because of an induced electric dipole moment, and from a nonzero mean squared charge radius $<\rho r^{2}>$. The latter is a lowest order term of the intrinsic neutron electron interaction seen on top of the Foldy interaction, which comes from the magnetic moment of the neutron. Both $\alpha_{n}$ and $<\rho r^{2}>$ change the scattering cross section $\sigma_{s}$ as much as 0.2 percent.

To determine $<\rho r^{2}>$, the corrected shape of $\sigma_{s}$ in first born approximation is $4 \pi\left(b_{N c}^{2}-\right.$ $\left.2 b_{N c}\left(b_{F}+b_{1}\right) Z(1-f(k))+\ldots\right) . \quad Z$ is the nuclear charge number and $f(k)$ the atomic form factor, $b_{N c}, b_{F}$ and $b_{1}$ are the scattering lengths of nuclear-, Foldy-, and intrinsic $n-\mathrm{e}^{-}$-interaction. From $b_{1}$ a value of $\left\langle\rho r^{2}\right\rangle$ is derived. Preliminary measurements have been made at ORELA to study count rates and backgrounds down to $0.15 \mathrm{eV}$ using a flight path length of $18 \mathrm{~m}$. Additional measurements using liquid $\mathrm{Pb}$ samples, are planned for December 1990. Results are expected for the date of the meeting.

Research sponsored by U.S. DOE Office of Energy Research, Division of Nuclear Physics.

${ }^{*}$ Institut fur Kernphysik der Tu Wien, Wien, Austria.

$\dagger_{\text {Instrumentation and Controls Division. }}$

2.13

\section{${ }^{10} B\left(n, \alpha_{1} \gamma\right){ }^{7} \mathrm{Li}$ CROSS SECTION BETWEEN 0.3 AND 4.0 MeV}

\author{
R. A. Schrack* O. A. Wasson* \\ D. C. Larson J. K. Dickens \\ J. H. Todd \\ [Abstract of Nucl. Sci. Eng. (in press)]
}

Relative cross-section measurements for the ${ }^{10} \mathrm{~B}\left(\mathrm{n}, \alpha_{1} \gamma\right){ }^{7} \mathrm{Li}$ reaction were made using the ORELA neutron source. The cross sections were measured by observing the $478-\mathrm{keV}$ photon using a 30\% "efficiency" intrinsic germanium detector. The neutron flux was monitored with a high efficiency plastic scintillator. Monte Carlo calculations were used to provide for multiple-scattering and neutron-attenuation corrections to the data. The measured cross sections differ as much as $40 \%$ from the ENDF/B-VI evaluation for incident neutron energies greater than $1.5 \mathrm{MeV}$.

Research sponsored by U.S. DOE Division of Nuclear Physics.

*National Institute of Standards and Technology, Gaithersburg, MD.

$\dagger_{\text {Instrumentation and Controls Division. }}$ 


\subsection{4}

\section{MEASUREMENT OF THE ${ }^{10} \mathrm{~B}(\mathrm{n}, \alpha \gamma)$ CROSS SECTION IN THE 0.3- TO 4-MeV NEUTRON ENERGY INTERVAL}

\author{
R. A. Schrack* \\ O. A. Wasson* \\ D. C. Larson \\ J. H. Todd ${ }^{\dagger}$ \\ J. K. Dickens
}

[Abstract of paper presented at the International Conference on Nuclear Data for Science and Technology, Julich, Germany, May 13-17, 1991; Proc. pp. 507-509, S. M. Qaim, Ed., Springer-Verlag (1992)]

This new measurement was performed in order to improve the accuracy of this important neutron cross section standard in the neutron energy region above $600 \mathrm{keV}$. The experiment was carried out at the $150-\mathrm{m}$ flight path of the ORELA pulsed neutron time-of-flight facility located at Oak Ridge National Laboratory. The relative cross section was measured in the neutron energy region from 0.3 to $4 \mathrm{MeV}$. A beryllium target neutron source was used to provide increased neutron flux at the high end of the neutron energy range and to reduce the $\gamma$-ray flash intensity. A high purity germanium detector having $30 \%$ efficiency was used to measure the $478-\mathrm{keV} \gamma$ ray from the ${ }^{10} \mathrm{~B}(\mathrm{n}, \alpha \gamma)$ reaction in the sample, which was positioned $19 \mathrm{~m}$ from the neutron source. The neutron fluence incident on the sample was determined from measurements using the NIST Black Detector which was positioned at $150 \mathrm{~m}$ on the same flight path. The efficiency and response of this detector have been carefully determined in a series of previous measurements and calculations at NIST. The neutron flight time and pulse amplitude for each event in both detectors were recorded in the new ORELA data acquisition system based on a microcomputer. The data reduction and analysis was performed at NIST using a newly written series of programs. The effects of neutron scattering in the boron sample have been extensively studied in the analysis. The absolute cross section, which was obtained from normalization to the ENDF/B-6 evaluation in the lower neutron energy region, will be presented and compared with previous measurements.

Research sponssred by U.S. DOE Office of Energy Research, Division of Nuclear Physics.

*National Institute of Standards and Technology, Gaithersburg, MD.

$\dagger_{\text {Instrumentation and Controls Division. }}$

2.15

\section{FAST NEUTRON-INDUCED CHANGES IN NET IMPURITY CONCENTRATION OF HIGH- RESISTIVITY SILICON}

\author{
I. Tsveybak* \\ W. Bugg* \\ J. A. Harvey \\ J. Walter ${ }^{\dagger}$
}

(Abstract of paper presented at the 1992 IEEE Annual
Conference on Nuclear and Space Radiation Effects, New
Orleans, LA, July 13-17, 1992; also Southeastern Section
Meeting of the American Physical Society, Oak Ridge, TN,
November 12-14, 1992; Proc. Vol. 37(7), p. 1675 (1992)]

Resistivity changes produced by $1 \mathrm{MeV}$ neutron irradiation at room temperature have been measured in float-zone grown $\mathrm{n}$ and p-type silicon with initial resistivities ranging from 1.8 to $100 \mathrm{k} \Omega^{*} \mathrm{~cm}$. Observed changes are discussed in terms of net electrically active impurity concentration. A model is presented which postulates escape of $\mathrm{Si}$ self-interstitials and vacancies from damage clusters and their subsequent interaction with impurities and other pre-existing defects in the lattice. Differences in the behavior of n-type and $p$-type material are explained on the basis of a faster removal of substitutional $P$ and a more nonuniform spatial distribution of the original $P$ concentration.

Research sponsored U.S. DOE Division of Nuclear Physics.

*University of Tennessee, Knoxville, TN.

$\dagger_{\text {IntraSpec Inc., Cak Ridge, TN. }}$

2.16

\section{IS THE PROBLEM WITH \\ Pu-239 FISSION CROSS SECTION SOLVED?}

\section{W. Weston J. A. Harvey H. Derrien*}

[Abstract of paper presented at the Southeastern Section Meeting of the American Physical Soriety, Oak Ridge, TN, November 12-14, 1992; Proc. Vol. 37(7), p. 1677 (1992)]

In the resolved resonance region of the $\mathrm{Pu}$ 239 fission cross section there has been a discrepancy in the measured average value and a discrepancy in the value of the cross sections between resonances. This was one of 6 major discrepancies in nuclear data singled out for study by an international committee. Very careful additional 
measurements and analysis have been carried out at ORELA and the problem is considered solved. Publications of the results are underway and the international committee has given preliminary approval of the results. The low fission cross sections between resonances which were previously reported in ORELA measurements have been confirmed both by new fission cross section measurements and by simultaneous fitting with results from ORELA transmission measurements. Very careful normalization measurements indicate that the previous ORELA fission measurements which were low compared to other data must be normalized upward by $3 \%$. This will bring most data sets into agreement within experimental uncertainties. This is an example of how nuclear data measurements at ORELA, with international cooperation, continue to eliminate important discrepancies.

Research sponsored U.S. DOE Division of Nuclear Physics.

* Japan Atomic Energy Research Institute, Japan.

2.17

\section{HIGH RESOLUTION FISSION CROSS SECTION MEASUREMENTS OF ${ }^{235} \mathrm{U}$ AND ${ }^{239} \mathrm{Pu}$}

\section{W. Weston J. H. Todd*}

[Abstract of Nucl. Sci. Eng. 111, 415 (1992)]

The fission cross sections of ${ }^{235} \mathrm{U}$ and ${ }^{239} \mathrm{Pu}$ are measured with very high neutron energy resolution $(0.17 \mathrm{~ns} / \mathrm{m})$ in the energy region from 100 to $2,000 \mathrm{eV}$ for ${ }^{235} \mathrm{U}$ and to $20,000 \mathrm{eV}$ for ${ }^{239} \mathrm{Pu}$. The purpose of this measurement is to provide fission cross sections with energy resolution comparable with that available from transmission measurements for the purpose of deriving multilevel resolved resonance parameters. Fission ion chambers are used to detect fission fragments, and a ${ }^{10} \mathrm{~B}$ ionization chamber is used to measure the relative neutron flux at the $86-\mathrm{m}$ flight path of the Oak Ridge Electron Linear Accelerator. The measured fission cross sections are the highest resolution measurements of good accuracy reported in the neutron energy range above $400 \mathrm{eV}$.

Research sponsored by U.S. DOE Division of Nuclear Physics.

*Instrumentation and Controls Division.
2.18

\section{R-MATRIX ANALYSIS OF AN ORELA MEASUREMENT OF THE $n+{ }^{48} \mathrm{Ca}$ TOTAL CROSS SECTION FROM $100 \mathrm{keV}$ TO $3.9 \mathrm{MeV}$}
R. F. Carlton*
C. H. Johnson ${ }^{\dagger}$

\section{J. A. Harvey}

(Abstract of paper presented at the 1992 Joint April Meeting of the American Physical Society and the American Association of Physics Teachers, Washington, DC, April 20-24, 1992; Proc. Vol. 37(2), p. 902 (1992)]

Earlier work predicted little s-wave strength in the region above $2 \mathrm{MeV}$, based on shell model in the continuum calculations. Only three narrow low energy resonances were consistent with a $1 / 2^{+}$assignment. With improved statistics we conclude only one of these is s-wave, and have identified a total of 9 below 4-MeV. The strength function based on the earlier work was $\approx 10 \%$ of the value expected. The current estimate is $(2.6 \pm 1.3) \times 10^{-4}$ in good agreement with systematic trends. We have made $\mathrm{J}^{\pi}$ assignments to $15 \mathrm{p}$-wave resonances and $38 \mathrm{~d}$-wave resonances. The $d_{5 / 2}$ interaction continues to dominate with a strength of $(10.7 \pm 1.3) \times 10^{-4}$. Assuming tnat the observed reduced neutron widths, $\gamma_{J \ell}^{2}$, are drawn from Porter-Thomas distributions with population means $<\gamma_{J \ell}^{2}>$, and are larger than $1 / 4$ that mean, we estimate that we have missed or spuriously identified $2-p_{1 / 2}, 7-p_{3} / 2,8-d_{3 / 2}$, and $2-d_{5 / 2}$ resonances. The observed level densities can be described using the back-shifted Fermi-gas model with density parameters, $a_{c}=3.98 \pm 0.14 \mathrm{MeV}^{-1}$ and $U_{0}=0.24 \pm 0.01 \mathrm{MeV}$.

Research sponsored by U.S. Department of Energy.

* Middle Tennessee State University, Murfreesboro, TN.

${ }^{\dagger}$ Consultant. 
2.19

\section{R-MATRIX ANALYSIS OF AN ORELA MEASUREMENT OF THE $\mathrm{n}+{ }^{208} \mathrm{~Pb}$ TOTAL CROSS SECTION FROM 78 TO $1700 \mathrm{keV}$}

\author{
R. F. Cariton* \\ J. A. Harvey \\ C. H. Johnson $\S$ J. Schmiedmayer \#
}

[Abstract of paper presented at the American Physical Society Spring Meeting, Washington, DC, April 21-25, 1991; Proc. Vol. 36(4), p. 1349 (1991)]

The energy resolution of the measurement is better than $1 / 5000$ which has allowed tentative $\mathrm{J} \ell$ assignments to be made for $13 \mathrm{~s}_{1 / 2}, 12 \mathrm{p}_{1 / 2}$, and $25 \mathrm{p}_{3 / 2}$ resonances. Assuming that the observed reduced neutron widths, $\gamma_{\lambda}^{2}(\mathrm{~J} \ell)$, are drawn from Porter-Thomas distributions with population means $\left\langle\gamma_{\lambda}^{2}(\mathrm{~J} \ell)\right\rangle$ and that the observed $\gamma_{\lambda}^{2}(\mathrm{~J} \ell)$ are larger than one-fourth the $\left\langle\gamma_{\lambda}^{2}(\mathrm{~J} \ell)\right\rangle$, we estimate that our preliminary analysis has missed or spuriously identified only one s-wave, perhaps as inany as $10 \mathrm{p}_{1 / 2}$, and not more than $3 \mathrm{p}_{3 / 2}$ resonances. The neutron strength functions, in conventional $10^{-4}$ units, derived from this work are $(0.71 \pm 0.30),(0.22 \pm 0.10)$, and $(0.31 \pm 0.09)$ for $s_{1 / 2}, p_{1 / 2}$, and $p_{3 / 2}$ partial waves. The observed $s_{1 / 2}, p_{1 / 2}$, and $p_{3 / 2}$ level densities can be described using the back-shifted Fermi-gas model with optimal density parameters, $a_{c}=10.84 \mathrm{MeV}$ and $\mathrm{U}_{o}=1.16 \mathrm{MeV}$.

Research sponsored by U.S. DOE Division of Nuclear Physics.

* Middle Tennessee State University, Murfreesboro, TN.

${ }^{\dagger}$ Denison University, Granville, $\mathrm{OH}$.

†Instrumentation and Controls Division.

$\S_{\text {Consultant. }}$

\# Technical University of Vienna.

2.20

\section{R-MATRIX ANALYSIS OF NEUTRON TOTAL CROSS SECTION DATA IN THE MeV REGION}

\author{
R. F. Carlton* J. A. Harvey \\ C. H. Johnson ${ }^{\dagger}$
}

[Abstract of paper presented at the Southeastern Section Meeting of the American Physical Socjety, Oak Ridge, TN, November 12-14, 1992; Proc. Vol. 37(7), p. 1676 (1992)]

Many complications are associated with the resonance analysis of neutron total cross section data. Even for spin-zero nuclei, making definite spin assignments is difficult above about one $\mathrm{MeV}$ for all but the lightest nuclei. Also, resonances outside the analyzed energy region are often crucial to the fits and assignments. Good fits to the data may be obtained with incorrect spin and parity assignments since interference between overlapping multiplets can be complex to decipher. Examples will be shown from measurements on ${ }^{48} \mathrm{Ca},{ }^{86} \mathrm{Kr}$, and ${ }^{208} \mathrm{~Pb}$. Techniques and methodologies for extracting $\mathrm{J}^{\pi}$-separated level densities, strength functions, scattering functions, and $\mathrm{R}$ functions have been developed from this source of information on the properties of states in the unbound region. These deduced quantities can be connected to models of nuclear structure.

Research sponsored by U.S. DOE Office of Energy Research, Division of Nuclear Physics.

*Middle Tennessee State University, Murfreesboro, TN.

†onsultant.

2.21

MULTILEVEL RESONANCE ANALYSIS OF ${ }^{59} \mathrm{Co}$ TRANSMISSION MEASUREMENTS

\section{G. de Saussure N. M. Larson*}

(Abstract of paper presented at the International Conference on Nuclear Data for Science and Technology, Julich, Germany, May 13-17, 1991; Proc. pp. 109-111, S. M. Qaim, Ed., Springer-Verlag (1992)]

Large discrepancies exist between the highresolution ${ }^{59}$ Co neutron transmission data of Harvey et al. and the resolved resonance parameters of ENDF/B-VI. In order to provide new resonance 
parameters consistent with these data, the highresolution transmission measurements have been analyzed with the computer code SAMMY. Results of that analysis are reported here.

Research sponsored by U.S. DOE Office of Energy Research, Division of Nuclear Physics.

* Computing and Telecommunications Division.

\subsection{2}

\section{MULTILEVEL RESONANCE ANALYSIS OF ${ }^{59}$ Co NEUTRON \\ TRANSMISSION MEASUREMENTS}

\author{
G. de Saussure \\ N. M. Larson* \\ J. A. Harvey \\ N. W. Hill ${ }^{\dagger}$
}

[Abstract of Ann. Nucl. Energy 19(7), 393 (1992)]

Large discrepancies exist between the recent high-resolution ORELA neutron transmission data of ${ }^{59} \mathrm{Co}$ and transmissions computed from the resolved resonance parameters of ENDF/B-VI. In order to provide new resonance parameters consistent with these data, the transmission measurements have been analyzed with the computer code SAMMY in the energy range $200 \mathrm{eV}$ to $100 \mathrm{keV}$. The resonance parameters reported in this paper provide an accurate total cross section from $10^{-5}$ $\mathrm{eV}$ to $100 \mathrm{keV}$ and correctly reproduce the thermal capture cross section. Thermal cross-section values and related quantities are also reviewed here.

Research sponsored by U.S. DOE Division of Nuclear Physics.

* Computing and Telecommunications Division.

$\dagger_{\text {Consultant. }}$

\subsection{3}

\section{MULTILEVEL RESONANCE ANALYSTS OF ${ }^{59} \mathrm{Co}$ NEUTRON TRANSMISSION MEASUREMENTS}
G. de Saussure
N. M. Larson*
J. A. Harvey
N. W. Hill ${ }^{\dagger}$

(Abstract of ORNL/TM-11762, May 1991)

High-resolution neutron transmission measurements through several thicknesses of ${ }^{59} \mathrm{Co}$ were performed in 1986 at the Oak Ridge Electron Linear Accelerator (ORELA) in conjunction with the ENDF/B-VI evaluation of ${ }^{59} \mathrm{Co}$ done at $\mathrm{Ar}$ - gonne National Laboratory. These measurements have much better energy resolution than previously published transmission measurements. The results of one of the measurements were used in the ENDF/B-VI evaluation of the total cross section above $100 \mathrm{keV}$, but the data were not used below $100 \mathrm{keV}$, where the ENDF/B-VI cross sections are represented by resolved resonance parameters based on a compilation of Mughabghab et al.

The transmission measurements are not very sensitive to the values of the resonance capture widths; therefore, the capture widths obtained in this analysis have large uncertainties. An attempt was made to adjust the capture widths to give capture areas consistent with the capture areas reported by Spencer et al. However, the attempt was not successful because the correspondence between the resonances observed in transmission and those observed in capture is not always unambiguous and because the capture areas measured by Spencer et al. often have large and uncertain multiple scattering contributions. Resonance parameters are required to properly estimate these contributions.

Research sponsored by U.S. DOE Division of Nuclear Physics.

*Computing and Telecommunications Division.

$\dagger_{\text {Instrumentation and Controls Division. }}$

\subsection{4}

EQUILIBRIUM AND PRE-EQUILIBRIUM MODELS FOR CALCULATION OF PARTICLE EMISSION CROSS SECTIONS AND SPECTRA

$$
\text { C. Y. Fu }
$$

(Abstract of paper presented at the Symposium on Nuclear Data Evaluation Methodology, Brookhaven National Laboratory, Upton, NY, October 12-16, 1992)

Improvements of equilibrium and preequilibrium models that have been applied to the evaluations of the recently released nuclear data libraries are reviewed. The improvements include energy-angle correlated neutron emission in the continuum, charged-particle and recoil spectra, widths-fluctuation correction for the continuum, pre-equilibrium capture, and gamma-ray strength functions. Their impacts on evaluated data are summarized.

Research sponsored by U.S. DOE Office of Energy Research, Division of Nuclear Physics. 
2.25

\section{CROSS SECTION \\ PARAMETERIZATION \\ IN THE RESOLVED \\ RESONANCE REGION}

\section{N. M. Larson*}

(Abstract of paper presented at the Symposium on Nuclear Data Evaluation Methodology, Brookhaven National Laboratory, Upton, NY, October 12-16, 1992)

Experimental techniques, methods, and equipment have evolved to provide more accurate neutron cross section data with better energy resolution. Keeping pace with those developments has been a challenge for data analysts; commensurate improvements in analysis tools are required. In this paper, analysis techniques for neutron timeof-flight data in the resolved resonance region are discussed, with emphasis on contemporary needs.

Research sponsored by U.S. DOE Office of Energy Research, Division of Nuclear Physics.

* Computing and Telecommunications Division.

\subsection{6}

\section{CALCULATION OF $(n, x \boldsymbol{\gamma})$ CROSS SECTIONS BETWEEN THRESHOLD AND $100 \mathrm{MeV}$ FOR Fe AND Pb ISOTOPES: COMPARISON WITH EXPERIMENTAL DATA}

\author{
P. G. Young* \\ R. O. Nelson* \\ C. M. Laymon* \\ D. M. Drake* \\ H. Vonach ${ }^{\dagger}$ \\ S. Tagesen ${ }^{\dagger}$ \\ D. S. Dale \\ R. C. Haight* \\ S. A. Wender* \\ G. L. Morgan* \\ M. Drosg ${ }^{*}$ \\ A. Pavlik \\ D. C. Larson
}

Abstract of paper presented at the International Atomic Energy Agency Final Meeting of a Co-ordinated Research Programms, Vienna, Austria, June 20-22, 1990; Proc. pp. 239-250 (1991)]

An experimental program is in progress at the Los Alamos National Laboratory WNR/LAMPF facility to perform high-resolution measurements of $(n, x \gamma)$ cross sections for individual lines up to incident neutron energies in the medium-energy range for a variety of target materials. Part of the purpose of these measurements is to provide a data base for testing the details of nuclear mod- els in this energy range, with the goal of facilitating model improvements. In this paper initial calculations using the GNASH nuclear theory code and the level density models of Gilbert-Cameron and Ignatyuk are described. The results are compared to the existing experimental data base for $204,206,207,208 \mathrm{~Pb}$ isotopes at lower energies and to preliminary data from the WNR/LAMPF measurements on ${ }^{\text {nat }} \mathrm{Fe}$ and ${ }^{208} \mathrm{~Pb}$ up to $E_{n}=100$ $\mathrm{MeV}$.

Research sponsored by U.S. DOE Office of Energy Research, Division of Nuclear Physics.

*Los Alamos National Laboratory, Los Alamos, NM.

$\dagger_{\text {University of Vienna, Austria. }}$

$\ddagger_{\text {Unives sity of Mlinois, Urbana, IL. }}$

\subsection{7}

\section{THEORETICAL CALCULATION OF NEUTRON INDUCED DATA OF ${ }^{19}$ F AND UNCERTAINTIES OF PARAMETERS}

\section{$\begin{array}{ll}\text { Z. Zhixiang* } & \text { C. Y. Fu }\end{array}$}

D. C. Larson

[Abstract of Chinese Journal of Nuclear Physics 14(1), 67 (1992)]

Nuclear model codes are used to calculate neutron induced data on ${ }^{19} \mathrm{~F}$ for incident energies from 2 to $20 \mathrm{MeV}$. The model parameters in the codes are adjusted to best reproduce experimental data. The calculated results are compared to the measured data and the evaluated values of ENDF/B-V. The covariance matrix for several of the most sensitive model parameters is given based on the scattering of the measured data around the theoretical curves and the long-range correlation error of the measured data. These results form the basis for the new ENDF/B-VI fluorine evaluation.

Research sponsored by U.S. DOE Division of Nuclear Physics.

* China Institute of Atomic Energy, China. 


\section{CROSS SECTION EVALUATION AND NUCLEAR MODELING}

\author{
INTERNATIONAL EVALUATION \\ COOPERATION TASK 1.1: \\ INTERCOMPARISON OF EVALUATED \\ FILES FOR ${ }^{52} \mathrm{Cr},{ }^{56} \mathrm{Fe}$, \\ AND ${ }^{58} \mathrm{Ni}$
C. Y. Fu
D. C. Larson
D. M. Hetrick*
J. Kopecky ${ }^{\ddagger}$
S. Iijima ${ }^{\S}$
N. Yamamuro\#
G. Maino** \\ H. K. Vonach ${ }^{\dagger}$
}

Abstract of paper presented at the International Conference on Nuclear Data for Science and Technology, Julich, Germany, May 13-17, 1991; Proc. pp. 857-859, S. M. Qaim, Ed., Springer-Verlag (1992)]

Task 1.1 (indicated in the title) is one of six initial tasks in a joint NEACRP/NEANDC Task Force on Evaluation Cooperation organized to promote international evaluation collaboration. The purpose of Task 1.1 is to graphically compare selected evaluations for structural materials in ENDF-VI (USA), JEF-II/EFF-II (Europe), and JENDL-III (Japan). The isotopes ${ }^{52} \mathrm{Cr},{ }^{56} \mathrm{Fe}$, and ${ }^{58} \mathrm{Ni}$ are especially interesting because they are important components of steel and because new evaluations for them have recently been completed for each library.

Large discrepancies have been found in some areas. For example, the spreads in the evaluated ${ }^{56}(\mathrm{n}, \alpha)$ cross sections around $10 \mathrm{MeV}$ and in the neutron emission spectra for ${ }^{58} \mathrm{Ni}$ for $E_{n}=8 \mathrm{MeV}$ and $E_{n}^{\prime}=1.5 \mathrm{MeV}$ are more than $30 \%$. Communications among the participants have been initiated for improving the evaluations in individual libraries, for assessing differences associated with present-day evaluation techniques, and for more consistent utilization of the formats.

Research sponsored by U.S. DOE Office of Energy Research, Division of Nuclear Physics.

*Computing and Telecommunications Division.

$\dagger$ Vienna University, Vienna, Austria.

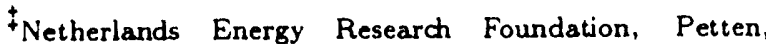
Netherlands.

$\S_{\text {Nuclear Engineering Laboratory, Kawasaki, Kanagawa, }}$ Japan.

\# Data Engineering, Inc., Yokohama, Kanagawa, Japan.

**ENEA, Bologna, Italy.
2.29

\section{TOOLS AND TECHNIQUES FOR EVALUATION INTERCOMPARISON}

\author{
D. M. Hetrick*
}

(Abstract of paper presented at the Symposium on Nuclear Data Evaluation Methodology, Brookhaven National Laboratory, Upton, NY, October 12-16, 1992)

Graphical comparison of cross sections computed from evaluations (ENDF/B-VI, JEF2/ EFF2, BROND, JENDL-3) versus measured data can be quite helpful in showing weaknesses in the evaluations, as well as uncovering discrepancies. A number of computer programs have been developed at the Oak Ridge Linear Accelerator (ORELA) facility that will read the appropriate data in ENDF format (V or VI) from the evaluations, perform the required calculations, and plot results versus available data. These codes have been used for comparison analyses of the structural material evaluations done at ORELA for version VI and are capable of plotting results for 1) reaction cross sections, 2) differential scattering cross sections, 3) angle-integrated emission spectra for neutrons, protons, alphas, etc., 4) doubledifferential cross sections of neutron emission, and 5) total secondary gamıma-ray spectra. The programs are written in FORTRAN, are interactive in that they prompt the user for information, and can be run either on a VAX mainframe computer with the results going to a plotter, or on an IBM cumpatible PC that is connected to a HewlettPackard (or compatible) laser printer. Plot files produced on the PC can be imported by a word processor. This paper describes these computer codes and the options that are available. Example plots are shown which compare evaluated cross sections with experimental data.

Research sponsored by U.S. DOE Office of Energy Research, Division of Nuclear Physics.

*Computing and Telecommunications Division. 
2.30

\section{APPROXIMATE METHODS FOR GENERATION OF COVARIANCE DATA FOR THE STRUCTURAL MATERIALS OF ENDF/B-VI}

\author{
$\begin{array}{ll}\text { D. M. Hetrick* } & \text { D. C. Larson }\end{array}$
}

C. Y. Fu

Abstract of paper presented at the Nuclear Energy Agency Nuclear Science Committee Specialists Meeting on Evaluation and Processing of Covariance Data, Oak Ridge National Laboratory, Oak Ridge, TN, October 7-9, 1992; Proc. pp. 173-182 (1993)]

The considerations that governed the development of cross-section uncertainty files for the isotopes of $\mathrm{Cr}, \mathrm{Fe}, \mathrm{Ni}, \mathrm{Cu}$, and $\mathrm{Pb}$ in ENDF/B-VI are summarized. Four different approaches were used in proviaing the uncertainty information. Illustrative examples are given which show the resulting standard deviations as a function of incident energy and the corresponding correlation matrices.

Research sponsored by U.S. DOE Office of Energy Research, Division of Nuclear Physics.

*Computing and Telecommunications Division.

\subsection{1}

\section{EVALUATION OF THE SILICON ISOTOPES FOR ENDF/B-VI}

\author{
D. M. Hetrick ${ }^{*}$ \\ D. C. Larson \\ N. M. Larson* \\ C. Y. Fu \\ S. J. Epperson ${ }^{\dagger}$
}

(Abstract of paper presented at the International Conference on Nuclear Data for Science and Technology, Julich, Germany, May 13-17, 1991; Proc. pp. 909-911, S. M. Qaim, Ed., Springer-Verlag (1992)]

Isotopic evaluations for ${ }^{28,29,30} \mathrm{Si}$ performed for ENDF/B-VI are briefly reviewed. The evaluations are based on analysis of experimental data and results of model calculations. Evaluated data are given for neutron induced reaction cross sections, angular and energy distributions, and gamma-ray production cross sections. All necessary data given to allow KERMA (Kinetic Energy Released in MAterials) and displacement cross sections to be calculated directly from information available in the evaluations. These quanti- ties are fundamental to studies of neutron heating and radiation damage.

Research sponsored by U.S. DOE Office of Energy Research, Division of Nuclear Physics

* Computing and Telecommunications Division.

$\dagger^{\dagger}$ University of Florida, Gainesville, FL.

2.32

\section{NEACRP/NEANDC WORKING GROUP ON INTERNATIONAL EVALUATION COOPERATION}

\author{
D. C. Larson C. L. Dunford* \\ C. Nordborg ${ }^{\dagger}$
}

Abstract of paper presented at the ANS Topical Meeting on New Horizons in Radiation Protection and Shielding, Pasco, WA, April 26-30, 1992; Proc. pp. 392-399 (1992)]

In the last three years, several newly evaluated nuclear data libraries have been released. Japan completed JENDL-3 in late 1989, JEF2/EFF-2 was completed by Europe in 1991, and ENDF/B-VI was completed by the U.S. in 1989. With the support of the NEACRP and the NEANDC, (recently combined into the NEA Nuclear Science Committee NEANSC) a Working Group was formed in 1989 to promote cooperative activities among the evaluation groups in OECD countries. Technical activities of the Working Group are carried out by subgroups formed to carry out specific investigations. Seven subgroups are currently active, with four more initiated by the Working Group at its meeting in May 1991. Brief descriptions of current subgroup activities are given.

Research sponsored by U.S. DOE Office of Energy Research, Division of Nuclear Physics.

*Brookhaven National Laboratory, Upton, NY.

$\dagger_{\text {NEA Data Bank, France. }}$ 
2.33

\section{POTENTIAL IMPROVEMENTS TO ENDF/B-VI FOR FUSION DATA}

\author{
D. C. Larson C. Y. Fu
}

Abstract of paper presented at the Topical Conference on Nuclear Data for Fusion Reactor Technology, Karlsruhe, Germany, October 23, 1991; Proc. pp. 113-118 (1992)]

While ENDF/B-VI is a significant improvement over previous versions for fusion reactor design calculations, several areas have not received attention and may need improvement. In addition, broadening of the contents of the evaluations to contain information not easily derived at present should be considered.

Research sponsored by U.S. DOE Division of Nuclear Physics.

\subsection{4}

\section{COVARIANCES AS INPUT TO AND OUTPUT FROM RESONANCE ANALYSES}

\section{N. M. Larson*}

Abstract of paper presented at the Nuclear Energy Agency Nuclear Science Committee Specialists' Meeting on Evaluation and Processing of Covariance Data, Oak Ridge, TN, October 7-9, 1992; Proc. pp. 221-236 (1993)]

Accurate data analysis requires understanding of the roles played by both data and parameter covariance matrices. In this paper the entire data reduction/analysis process is examined, for neutron-induced reactions in the resonance region. Interrelationships between data and parameter covariance matrices are examined and alternative reduction/analysis methods discussed.

Research sponsored by U.S. DOE Office of Energy Research, Division of Nuclear Physics.

*Computing and Telecommunications Division.
2.35

COVARIANCE METHODS FOR

R. W. Peelle

(Abstract of paper presented at the Symposium on Nuclear Data Evaluation Methodology, Brookhaven National Laboratory, Upton, NY, October 12-16, 1992)

Neutron cross-section standards evaluations differ from most others in that more accurate results are expected and in that a greater number and variety of interdependent measurements should be included. Therefore, cross sections and covariances for several reactions need to be evaluated in the same procedure. The constraints of reaction theory should be included. The multiphase work of the Standards Subcommittee of the Cross Section Evaluation Working Group provides examples of problems encountered in such covariance analyses, and in some cases approaches available to solve them. The small output uncertainties that are characteristic of such data combination projects are discussed to help reconcile them with the qualitative impression of experts that larger uncertainties actually prevail. For those cross sections constrained by reaction theory, improved techniques are needed to treat energy regions where existing data are inconsistent with plausible resonance structures. Suggestions are included for covariance methods that might be employed in future standards evaluations.

Research sponsored by U.S. DOE Office of Energy Research, Division of Nuclear Physics.

2.36

\section{THE RESOLUTION OF DISCREPANCIES AMONG NUCLEAR DATA}

\author{
R. W. Peelle
}

(Abstract of paper presented at the Symposium on Nuclear Data Evaluation Methodology, Brookhaven National Laboratory, Upton, NY, October 12-16, 1992)

Significant differences among input data occur in the evaluation of nuclear data because it is difficult to achieve experimental results with the accuracy required for some applications. Types of "discrepancies" are classified. The means are reviewed by which an evaluator may treat discrepan- 
cies in the process of evaluation. When all means fail that are based on how the discrepant data were obtained, the perplexed evaluator must sometimes combine discrepant data based just on the stated values and uncertainties; techniques for treating such challenges are compared. Some well-known data discrepancies are examined as examples.

Research sponsored by U.S. DOE Office of Energy Research, Division of Nuclear Physics.

\subsection{7}

\section{APPROXIMATE METHODS FOR DERIVATION OF COVARIANCE DATA}
S. Tagesen*
D. C. Larson

(Abstract of paper presented at the Symposium on Nuclear Data Evaluation Methodology, Brookhaven National Laboiatory, NY, October 12-16, 1992)

Several approaches for the derivation of covariance information for evaluated nuclear data files (EFF-2 and ENDF/B-VI) have been developed and used at IRK and ORNL respectively. Considerations are presented which govern the choice of a particular method depending on the quantity and quality of available data. Advantages/disadvantages are discussed and examples of results are given.

Research sponsored by U.S. DOE Office of Energy Research, Divisian of Nuclear Physics.

* University of Vienna, Vienna, Austria.

2.38

COVARIANCE MATRIX OF MODEL PARAMETERS AND UNCERTAINITIES OF CALCULATED NEUTRON CROSS SECTIONS ON ${ }^{10} \mathrm{~F}$

\section{$\begin{array}{ll}\text { Z. Zhao* C. Y. Fu } & \text { C. }\end{array}$ D. C. Larson}

(Abstract of paper presented at the Nuclear Energy Agency Nuclear Science Committee Specialists' Meeting on Evaluation and Processing of Covariance Data, Oak Ridge, TN, October 7-9, 1992; Proc. pp. 71-75 (1993)]

A simplified method to estimate covariance matrix of the model parameters is presented in this work and applied to calculate covariance matrix of calculated cross sections on ${ }^{19} \mathrm{~F}$.

Research sponsored by U.S. DOE Office of Energy Research, Division of Nuclear Physics.

*Institute of Atomic Energy, Beijing, China.

2.39

THE COVARIANCE MATRIX
OF DERIVED QUANTITIES
AND THEIR COMBINATION

$$
\text { Z. Zhao* F. G. Perey }
$$

(Abstract of ORNL/TM-12106, June 1992)

The covariance matrix of quantities derived from measured data via nonlinear relations are only approximate since they are functions of the measured data taken as estimates for the true values of the measured quantities. The evaluation of such derived quantities entails new estimates for the true values of the measured quantities and consequently implies a modification of the covariance matrix of the derived quantities that was used in the evaluation process. Failure to recognize such an implication can lead to inconsistencies between the results of different evaluation strategies. In this report we show that an iterative procedure can eliminate such inconsistencies.

Research sponsored by U.S. DOE Division of Nuclear Physics.

*Institute of Atomic Energy, Beijing, China.

\subsection{0}

\section{TIME DEPENDEN'T MONTE CARLO CALCULATIONS OF THE ORELA TARGET NEUTRON SPECTRUM}

\author{
S. N. Cramer F. G. Perey
}

[Abstract of Nucl. Sci. Eng. (in press)]

The time dependent spectrum of neutrons in the water-moderated Oak Ridge Electron Linear Accelerator (ORELA) target has been calculated using a modified version of the MORSE multigroup Monte Carlo code with an analytic hydrogen scattering model. Distributions of effective neutron distance traversed in the target are estimated with a time and energy dependent algorithm from the leakage normal to the target face. These data are used in the resonance shape analy. ses of time-of-flight cross section measurements to account for the experimental resolution function. The $20 \mathrm{MeV}-10 \mathrm{eV}$ energy range is adequately represented in the MORSE code by the 174 group VITAMIN-E cross section library with a $\mathrm{P}_{5}$ expansion. An approximate representation of the ORELA positron source facility, recently installed near the target, has been included in the calcula- 
tions to determine any perturbations the positron source might create in the computed neutron distributions from the target. A series of coupled Monte Carlo calculations was performed from the target to the positron source and back to the target using a next-event estimation surface source for each step. The principal effect of the positron source was found to be an increase in the distance for the lower energy neutron spectra, producing no real change in the distributions where the ORELA source is utilized for experiments. Different configurations for the target were investigated in order to simulate the placement of a shadow bar in the neutron beam. These beam configurations included neutrons escaping from: (1) the central tantalum plates only, (2) the entire target with the tantalum plates blocked out, and (3) only a small area from the water. Comparisons of the current data with previous calculations having a less detailed model of the tantalum plates have been satisfactory.

Research sponsored by U.S. DOE Office of Nuclear Physics.

\subsection{1}

\section{COMPUTED SECONDARY-PARTICLE ENERGY SPECTRA FOLLOWING NONELASTIC NEUTRON INTERACTIONS WITH ${ }^{12} \mathrm{C}$ FOR E $_{n}$ BETWEEN 15 AND $60 \mathrm{MeV}$ : COMPARISONS OF RESULTS FROM TWO CALCULATIONAL METHODS}

\section{J. K. Dickens}

\section{(Abstract of ORNL/TM-11812, April 1991)}

The organic scintillation detector response code SCINFUL has been used to compute secondary-particle energy spectra, $d \sigma / d E$, following nonelastic neutron interactions with ${ }^{12} \mathrm{C}$ for incident neutron energies between 15 and $60 \mathrm{MeV}$. The resulting spectra are compared with published similar spectra computed by Brenner and Prael who used an intranuclear cascade code, including alpha clustering, a particle pickup mechanism, and a theoretical approach to sequential decay via intermediate particle-unstable states. The similarities of and the differences between the results of the two approaches are discussed.

Research sponsored by U.S. DOE Division of Nuclear Physics.

2.42

\section{SCINTILLATION DETECTOR EFFICIENCIES FOR NEUTRONS IN THE ENERGY REGION ABOVE $20 \mathrm{MeV}$}

\author{
J. K. Dickens
}

(Abstract of paper presented at the Nuclear Energy Agency Nuclear Data Committee Specialists' Meeting on Neutron Cross Section Standards for the Energy Region Above $20 \mathrm{MeV}$, Uppsala, Sweden, May 21-23, 1991; Proc. NEANDC-305 "U" Organization for Economic Cooperation and Development, pp. 142-153 (1991)]

The computer program SCINFUL (for SCINtillator FULl response) is a program designed to provide a calculated complete pulse-height response anticipated for neutrons being detected by either an NE-213 (liquid) scintillator or an NE-110 (solid) scintillator in the shape of a right circular cylinder. The point neutron source may be placed at any location with respect to the detector, even inside of it. The neutron source may be monoenergetic, or Maxwellian distributed, or distributed between chosen lower and upper bounds. The calculational method uses Monte Carlo techniques, and it is relativistically correct. Extensive comparisons with a variety of experimental data have been made. There is generally overall good agreement (less than 10\% differences) of results from SCINFUL calculations with measured integral detector efficiencies for the design incident neutron energy range of 0.1 to $80 \mathrm{MeV}$. Calculations of differential detector responses, i.e., yield versus response pulse height, are generally within about $5 \%$ on the average for incident neutron energies between 16 and $50 \mathrm{MeV}$ and for the upper $70 \%$ of the response pulse height. For incident neutron energies between 50 and $80 \mathrm{MeV}$, the calculated shape of the response agrees with measurements, but the calculations tend to underpredict the absolute values of the measured responses. Extension of the program to compute responses for incident neutron energies greater than $80 \mathrm{MeV}$ will require new experimental data on neutron interactions with carbon.

Research sponsored by U.S. DOE Office of Energy Research, Division of Nuclear Physics. 
2.43

\section{CURRENT STATUS AND PROPOSED IMPROVEMENTS TO THE \\ ANSI/ANS-5.1 AMERICAN NATIONAL STANDARD FOR DECAY HEAT POWER IN LIGHT WATER REACTORS}

\section{J. K. Dickens $\quad$ T. R. England*} R. E. Schenter ${ }^{\dagger}$

[Abstract of Nuclear Safety 32(2), 209 (1991)]

The American National Standard for Decay Heat Power in Light Water Reactors (ANSI/ANS5.1) was issued in 1979 and has been used extensively for the past decade. Since the standard was issued, there have been new decay-heat measurements, and new and improved calculational capabilities have been developed. In this article the standard is compared with these new data and with calculations based on improved methods. Three foreign standards or proposed standards have also been developed, and their contents are compared with the present American standard. Proposals for improving the standard are presented and discussed.

Research sponsored by U.S. DOE Division of Nuclear Physics.

*Los Alamos National Laboratory, Los Alamos, NM.

${ }^{\dagger}$ Westinghouse Hanford Company, Richland, WA.

\subsection{4}

\section{ANALYSES OF PHYSICS SPECIMENS \\ IN FUEL PINS 1 AND 2 IRRADIATED IN THE DOUNREAY PROTOTYPE FAST REACTOR}

\author{
S. Raman* \\ B. L. Broadhead ${ }^{\dagger}$ \\ J. K. Dickens \\ J. L. Botts ${ }^{\ddagger}$ \\ R. L. Walker ${ }^{\ddagger}$ \\ (Abstract of ORNL-6632, January 1992)
}

The United States and the United Kingdom have been engaged in a joint research program in which samples of fissile and fertile actinides have been incorporated into three separate fuel pins and irradiated in the Dounreay Prototype Fast Reactor in Scotland. The actinides in the second fuel pin were studied for fission-product decay, specifically to obtain absolute yields of ${ }^{137} \mathrm{Cs}$. Comparisons with calculated yields result in ratios of measured to calculated between $0.67( \pm 0.05)$ and 1.09 ( \pm 0.18$)$. Plotting of experimental versus calculated values of ${ }^{137} \mathrm{Cs}$ indicated that the assumed flux levels were some 5 to $20 \%$ overestimated. This fiux level information will be useful in the forthcoming analysis of the last fuel pin, FP-4.

Research sponsored by U.S. DOE Office of Reactor Systems and Technology.

*Physics Division.

${ }^{\dagger}$ Computing and Telecommunications Division.

$\ddagger_{\text {Analytical Chemistry Division. }}$ 
Section 3

INTELLIGENT SYSTEMS 


\title{
3.0. INTRODUCTION
}

\author{
R. C. Mann
}

Research activities in the Intelligent Systems Section (ISS) concentrate on a broad spectrum of challenging areas ranging from intelligent interfaces between humans and complex systems to autonomous mobile robots, as well as biomedical applications of advanced pattern recognition systems. The section comprises the Autonomous Robotic Systems Group, the Adaptive Algorithms and Sensor Systerns Group, the Informatics Group and the Cognitive Systems and Human Factors Group.

The Center for Engineering Systems Advanced Research (CESAR), sponsored by the Engineering Sciences Program of the Department of Energy (DOE) Office of Basic Energy Sciences, represents the section's core long-term basic research program in intelligent machines. CESAR research includes studies in multiple cooperating robots, multi-sensor data analysis and fusion, control of mobile robots and manipulators, machine learning, and embedded high performance computing. With support from the DOE Office of Nuclear Energy, the ISS has been performing applied robotics research, systems integration, and has provided overall coordination and management of a consortium of four university research groups (Florida, Michigan, Tennessee, Texas) in a program aimed at robotics for advanced nuclear power stations. The ISS also contributed unique expertise to the DOE Office of Nuclear Energy Advanced Controls Program for tasks related to cognitive modeling and experimental human performance research. Advanced intelligent machine and robotics technology development was also performed in the ISS for a large multi-laboratory program in robotics for environmental restoration and waste management funded by the DOE Office of Environmental Restoration and Waste Management. Non-DOE sponsors of research in the ISS include the Department of Defense, the National Highway Transportation Safety Administration, the Federal Highway Administration and the Nuclear Regulatory Commission.

The section has outstanding facilities that support experimental research in autonomous and human-machine systems, as well as human performance experimental investigations. The CESAR laboratory with its mobile robots (HERMIES-IIB, HERMIES-III, a new experimental platform, and a commercially available mobile robot, ANDROS), redundant degrees-of-freedom research manipulators, laser range cameras, multi-spectral vision system, and computer network including hypercube concurrent processors, parallel systolic arrays, custom-made VLSI fuzzy logic processors, and scientific workstations, continues to be used as a collaborative research facility by numerous guest researchers and students from the U.S. and overseas (Japan, France, Germany, Denmark, Norway, Belgium). Our Cognitive Engineering Research Laboratory currently conducts eye-gaze experiments to support cognitive modeling and human performance research, and "natural" means for human-in-the-loop processing.

Significant technical progress was achieved in a number of areas. The following paragraphs briefly summarize a number of highlights: 
Autonomous Robotic Systems Group

The effective use of redundant manipulators mounted on mobile platforms represents challenging fundamental problems ranging from high-level planning issues to sensor-based control of the highly redundant overall robot system. Several optimization methods have been developed to derive optimal configurations of platformmanipulator systems during task commutation when force and position constraints are imposed at the end-effector, and obstacle avoidance, maneuverability, and joint torque constraints exist on the system.

An integrated proof-of-principle experiment involving surface-following by the kinematically redundant CESARm manipulator using an end-effector mounted sensor and with simultaneous motion of the platform has been performed. The goal of this successful experiment has been to demonstrate the feasibility of the integrated sensor-driven combined motion of the platform and manipulator between optimized configurations.

A novel redundancy resolution method using the combinatorial linearity of submatrix's solutions has been developed and tested using graphics simulations of the CESARm redundant manipulator.

Fundamental path planning problems arise with robots for which mobility is subject to kinematic constraints, e.g., car-like robots. Such systems have received little attention from the robotics research community, yet are very important for a number of applications, including environmental restoration, waste management, intelligent vehicle/highway systems, and planetary exploration. A geometric reasoning approach has been developed which allows precise calculation of constrained system trajectories, and a corresponding algorithm for car-like robot path planning in complex environments has been implemented.

Another approach, inspired from the qualitative reasoning formalism which we have developed, has been used to investigate two control modes for the navigation of car-like robots in a priori unknown environments. In the first mode, the car navigates fully autonomously, while in the second mode, the sensor-based reasoning system acts as a driver's aid providing the driver with linguistic-type commands depending on the perceived environment. Proof-of-principle experiments have been performed using a real car in outdoor environments to illustrate the feasibility and robustness of the proposed approach for outdoor autonomous navigation and/or safety enhancing driver's aid.

A new mobility concept based on a novel wheel assembly was recently developed. The concept, which provides a full omnidirectional and holonomic motion capability to mobile platforms, has been demonstrated through a prototype system, and a patent application has been filed with the U.S. Patent Office.

One of the greatest challenges in developing autonomous mobile robots that are planned to work in a priori unknown or dynamic environments is to design the reasoning modules allowing the robot to handle and/or cope with the many imprecisions, inaccuracies, and uncertainties that are always present in the real world. Unique computer boards incorporating custom-designed VLSI fuzzy inferencing chips have recently been developed to add a qualitative reasoning capability to real-time control systems, allowing implicit incorporation of uncertainties in the decision making process of autonomous robots. 
An approach using superposition of elemental sensor-based behaviors has been proposed for the development and testing of qualitative reasoning schemes emulating human-like inferencing behaviors. This approach has recently been tested in experiments involving mobile robots navigating in a priori unknown, indoor and outdoor, environments on the basis of very sparse and inaccurate sensor data.

A control architecture which decouples the force- and position-controlled degrees-of-freedom during motion of constrained closed-chain systems has been developed. The architecture has been applied to control of (1) the closed-chain motion of two dissimilar manipulators holding several kinds of common objects and (2) vehicles with multiple steerable wheels.

An approach which allows to transfortn the underspecified problem of solving for the joint velocities of a kinematically redundant manipulator into a well specified problem has been implemented on the seven degree-of-freedom CESARm. The redundancy resolution is accomplished by augmenting the original model with additional equations which define a new vector variable as a linear function of the joint velocities. Methods for selecting the redundant degree of freedom quantifying variable and the transformation matrix relating it to the joint velocities have been developed to yield a minimum Euclidean norm solution for the joint velocities.

The problem of modeling the error between the desired and actual orientations of a manipulator end-effector is complicated by the fact that Cartesian angular velocity is not the time derivative of a vector. We have derived a new model of the orientation error using Rodriguez parameters. We have determined the analytical time derivatives of the Ickes, Goldstein, and Rodriguez errors, and combined them with the translational motion error expression to obtain distinct closed loop kinematic velocity models using normalized quaternion feedback. Experimental verification of the orientation control techniques has been carried out on the seven degrees of freedom CESARm.

A decentralized control approach for mobile robots with differing characteristics (regarding sensors and mobility capability) has been developed. Experiments through computer simulations of the models have been performed, including the planning of time-minimal motions among moving obstacles. These results were published as part of a monograph book.

An approach to multi-sensor data integration involving pixel-level affinity functions to evaluate small neighborhood properties within each sensor image has been developed. Genetic algorithms are used to optimize the set of parameters controlling and weighting the various affinity functions based on each sensor's relative performance with respect to the current perception circumstances and user-defined objectives. This optimization method provides an adaptive capability which can be used for rapid domain switching and/or analysis of dynamic environments with constant sub-features.

\section{Adaptive Algorithms and Sensor Systems Group}

Machine learning research resulted in implementation of a random set algorithm based on Valiant's probabilistic learning theory. Results indicate that it performs at near optimal efficiency for discrete space problems. In addition, the N-learners problem has been studied as a means of further improving algorithmic efficiency. Several theorems were proven which indicate that under rather broad conditions, 
a fusion of $\mathrm{N}$ independent learners can perform better than any of the individual learners. This conclusion has been validated for a cluster perceptrons.

Neural networks are also being developed to process detector data and recognize low-probability nuclear reactions in the Superconducting Super Collider (SSC). In particular, two neural network algorithms were developed that reconstruct charged particle tracks through high energy detector systems. Extensive analysis showed that a feedforward neural network can identify two jet events from background events at an $~ 90 \%$ accuracy level. The neural network identification is based on the global distribution of energy that is deposited in the calorimeter of a high energy physics detector. It was also shown that a feedforward neural network can identify hadrons from muons in a high energy physics detector's calorimeter and muon chamber subsystems with an accuracy $>99 \%$. This technique exceeds all discrimiante analyses currently used. It is equivalent to the Bayes optimal discriminant.

In the DOE/NE Robotics for Advanced Reactors Program, we developed a design for remotely inspecting the passive cooling system of General Electric's Advancer Liquid Metal Reactor (ALMR). The designs produced by ISS and it's four university team members are currently being assembled into prototypes and have already been incorporated into the ALMR design. It is expected that these designs will permit the inspection of the passive cooling system without exposing human workers to high temperature or radiation levels. The use of robotics in this design is a key feature in assuring the economic viability of the ALMR.

Under the same program, ISS researchers assisted the Universities of Michigan and Tennessee in developing the control system for the team's entry in the American Association of Artificial Intelligence Robot Olympics. During this competition, Michigan's CARMEL robot competed against entries from MIT, IBM, the MITRE Corporation, and Carnegie Mellon University in obstacle avoidance, mapping, and goal finding exercises. The team's entry was the only one to successfully complete the entire course.

Experiments with our 16 processor Intel iWarp parallel computer have been directed towards construction of three dimensional environmental representations suitable for mobile robot navigation. These representations are based upon analysis of data from an imaging laser radar system. The implemented system achieves the performance goals of constructing and rendering a three dimensional occupancy map in less than the 800 millisecond frame time of the range camera. Initial comparisons of a traditional message-based communication algorithm and a particular systolic communication algorithm indicate that the latter provides superior performance by roughly a factor of two.

Results in sensor fusion have been obtained with a newly developed algorithm. This algorithm combines simulated annealing techniques with maximum entropy principles to fuse range and reflectance images from a laser range camera. The use of this algorithm significantly improves the quality of the range image compared to the unfused image. Specifically, range-reflectance crosstalk and range artifacts due to low reflectance objects were removed. The range and reflectance images were cooperatively fused through coupling between Markov random field lattices.

For applications involving hazardous environments, an ANDROS Mark VI robot has been procured and is undergoing conversion to computer control. The application of computer control to an industrial robot is expected to achieve much greater 
efficiency and safety in completing remote maintenance and inspection tasks in hazardous environments.

\section{Cognitive Systems and Human Factors Group}

Significant progress was made in research associated with the utilization of eyegaze technologies to effect "natural" interfaces. This past years work has dealt primarily with the search for implicit eye-gaze cues that would support a more natural control of camera position/point-of-regard. Telerobotic camera management could be an initial application.

An initial upgrade of the Nuclear Regulatory Commission's (NRC's) NUREG0700 (Guidelines For Control Room Design Evaluation) to account for digital technologies was completed.

An important and cornerstone project for the National Highway Transportation Safety Administration's (NHTSA's) Office of Crash Avoidance Research (OCAR) was initiated. This project involves the development of a driving-data acquisition system (collecting data on human performance, driving quality, environmental conditions and vehicle performance) that will be utilized in most of OCAR's planned experimental research over the next ten years. Initial efforts involved the identification of performance measures to be tracked by the system, including various types of physiological parameters. All data collection must be accomplished in a non-intrusive manner in order to ensure that driving performance is not affected by the data collection.

A front-end feasibility study was completed for the Federal Highway Administration (FHWA) that addressed the development of an Advanced Traffic Management System (ATMS) simulator, to be utilized for training and for the evaluation of emerging ATMS and Advanced Traveler Information System (ATIS) technologies. A needs analysis and cost-benefit analysis were a part of this activity. It was recommended that FHWA pursue the development of a prototype of such a simulator.

Support was provided for three activities associated with the Advanced Neutron Source (ANS). These were: 1) the preparation of an ANS human factors engineering plan (covering activities from conceptual design through construction and testing and evaluation), 2) the preparation of a document dealing with the intended operating philosophy of the ANS, and 3) the preparation of Chapter 18 of the ANS Conceptual Safety Analysis Report dealing with human factors support in the safety design of the ANS. The group conducted a study of the roles of human operators in existing and future nuclear reactor designs.

\section{Informatics Group}

In collaboration with biophysicists and molecular biologists at ORNL, research in the Informatics Group has been focusing to date on pattern recognitior- and parallel computing for DNA sequence analysis. The algorithms (GRAIL) developed so far are being used by a growing number of researchers world-wide (through internet) to analyze rapidly growing genome data sets. GRAIL, a multi-sensor/neural network pattern recognition system that integrates syntactic and statistical approaches, won a 1992 RD100 award. In addition to research and development, the group provides all information processing, data analysis, and database support for the ORNL 
Mouse Genome Center. Activities in the Informatics Group are increasing in depth and scope and include protein structure and function prediction studies, as well as other priority problems in the computat: nnal biosciences arena, and applications in large-scale information processing, filtering, and visualization. 


\section{ROBOTICS AND INTELLIGENT SYSTEMS}

\section{1}

\section{CESAR ROBOTICS AND INTELLIGENT SYSTEMS RESEARCH FOR NUCLEAR ENVIRONMENTS}

\author{
R. C. Mann
}

[Abstract of paper presented at the Specialist Meeting on Application of Artificial Intelligence and Robotics to Nuclear Plants (AIR '92), Ibaraki-ken, Japan, May 25-27, 1992; Proc. pp. 110-119 (1992)]

The Center for Engineering Systems Advanced Research (CESAR) at the Oak Ridge National Laboratory (ORNL) encompasses expertise and facilities to perform basic and applied research in robotics and intelligent systems in order to address a broad spectrum of problems related to nuclear and other environments. For nuclear environments, research focus is derived from applications in advanced nuclear power stations, and in environmental restoration and waste management. Several programs at CESAR emphasize the crosscutting technology issues, and are executed in appropriate cooperation with projects that address specific problem areas. Although the main thrust of the CESAR long-term research is on developing highly automated systems that can cooperate and function reliably in complex environments, the development of advanced human-machine interfaces represents a significant part of our research.

The objective of this paper is to review recent results of research and development at CESAR, with particular emphasis on activities targeted at robots for nuclear environments.

Research sponsored by U.S. DOE Office of Basic Energy Sciences.
3.2

\section{ROBOTICS AND INTELLIGENT SYSTEMS RESEARCH AT THE OAK RIDGE NATIONAL LABORATORY}

\author{
F. G. Pin
}

[Abstract of Robotics and Autonomous Systems 10(2), 81 (1992)]

The Oak Ridge Naticnal Laboratory (ORNL) located in Oak Ridge, Tennessee, is one of the major laboratories of the U.S. Department of Energy. As such, ORNL's mission is to pursue research and development activities in a wide spectrum of energy-related fields, with emphasis on energyproducing and utilization technologies, plants productivity and operation safety. Within this context, the area of robotics and intelligent systems is quite active because of the potential it represents for increasing overall productivity through automation but also, and perhaps more importantly, for improving overall safety by replacing humans in environments that are very hostile or hazarouds. In this editorial, the various facilities of the ORNL's Robotics and Intelligent Systems Program are introduced, and the variety of research themes and projects are reviewed. Eight papers, constituting a small but representative sample of ORNL's recent activities in Robotics and Intelligent systems, are then introduced.

Research sponsored by U.S. DOE Engineering Research Program of the Office of Basic Energy Sciences. 
3.3

\section{PROCEEDINGS OF THE SIXTH \\ INTERNATIONAL SYMPOSIUM ON METHODOLOGIES FOR INTELLIGENT SYSTEMS}

\section{K. S. Harber}

(Abstract of ORNL/TM-11938, September 1991)

This volume contains papers which have been selected for the Poster Session at the Sixth International Symposium on Methodologies for Intelligent Systems - ISMIS'91, held in Charlotte, North Carolina, October 16-19, 1991. The Symposium was hosted by UNC-Charlotte and sponsored by IBM-Charlotte, ORNL/CESAR, and UNC-Charlotte. These contributed papers have been selected from 55 full draft papers from the following major areas: (1) Expert Systems, (2) Intelligent Databases, (3) Knowledge Representation, (4) Learning and Adaptive Systems, and (5) Logic for Artificial Intelligence.

Research sponsored by U.S. DOE Office of Basic Energy Sciences.

\section{4}

\section{RESEARCH AND DEVELOPMENT AT ORNL/CESAR TOWARDS COOPERATING ROBOTIC SYSTEMS FOR HAZARDOUS ENVIRONMENTS}

\section{R. C. Mann K. Fujimura M. A. Unseren}

[Abstract of paper presented at the Fifth Annual Workshop on Space Operations, Applications, and Research (SOAR '91), NASA/Johnson Space Center, Houston, TX, July 9 11, 1991; Proc. NASA CP-3127, Vol. I, pp. 402-406 (1991)]

One of the frontiers in intelligent machine research in the understanding of how constructive cooperation among multiple autonomous agents can be effected. The effort at the Center for Engineering Systems Advanced Research (CESAR) at the Oak Ridge National Laboratory (ORNL) focuses on two problem areas: (1) cooperation by multiple mobile robots in dynamic, incompletely known environments; and (2) cooperating robotic manipulators. Particular emphasis is placed on experimental evaluation of research and developments using the CESAR robot system testbeds, including three mobile rotiots, and a seven-axis. kinematically redundant mobile manipulator. This paper summarizes initial results of research addressing the decoupling of position and force control for two manipulators holding a common object, and the path planning for multiple robots in a common workspace.

Research sponsored by U.S. DOE Office of Basic Energy Sciences.

\section{5}

\section{A NEW WHEEL CONTROL SYSTEM FOR THE OMNIDIRECTIONAL HERMIES-III ROBOT}

\section{B. Reister}

[Abstract of Robotica 10(4), 351 (1992); also ORNL/TM11784. April 1991; also paper presented at the 1991 IEEE International Conference on Robotics and Automation, Sacramento, CA, April 7-12, 1991; Proc. Vol. 3, pp. 23222327 (April 1991)]

We have designed, built, and tested a new wheel control system for the HERMIES-III robot. HERMIES-III is a large mobile robot with omnidirectional steering that is designed for human scale experiments. During each cycle (at $20 \mathrm{~Hz}$ ), the wheel control system moves the rotot toward a goal and calculates the current position of the robot. The system has seven modes for moving to a goal and the goal may be changed during motion of the robot.

Research sponsored by U.S. DOE Engineering Research Program of the Office of Basic Energy Sciences. 
3.6

\section{TIME OPTIMAL TRAJECTORIES FOR MOBILE ROBOTS WITH TWO INDEPENDENTLY-DRIVEN WHEELS}

\section{B. Reister F. G. Pin}

[Abstract of International Journal of Robotics Research (in press); also ORNL/TM-12070, March 1992]

This paper addresses the problem of timeoptimal motions for a mobile platform in a planar environment. The platform has two non-steerable independently driven wheels. The overall mission of the robot is expressed in terms of a sequence of via points at which the platform must be at rest in a given configuration (position and orientation). The objective is to plan time-optimal trajectories between these configurations assuming an unobstructed environment.

Using Pontryagin's maximum principle (PMP), we formally demonstrate that all time optimal motions of the platform for this problem occur for bang-bang controls on the wheels (at each instant, the acceleration on each wheel is either at its upper or lower limit). The PMP, however, only provides necessary conditions for time optimality. To find the time optimal robot trajectories, we first parameterize the bang-bang trajectories using the switch times on the wheels (the times at which the wheel accelerations change sign). With this parameterization, we can fully search the robot trajectory space and find the switch times that will produce particular paths to a desired final configuration of the platform. We show numerically that robot trajectories with three switch times (two on one wheel, one on the other) can reach any position, while trajectories with four switch times can reach any configuration. By numerical comparison with other trajectories involving similar or greater numbers of switch times, we then identify the sets of time-optimal trajectories. These are uniquely defined using ranges of the parameters, and consist of subsets of trajectories with three switch times for the problem when the final orientation of the robot is not specified, and four switch times when a full final configuration is specified. We conclude with a description of the use of the method for trajectory planning for one of our robots, and discuss some comparisons of sample time-optimal paths with minimum-length paths.

Research sponsored by U.S. DOE Engineering Research Program of the Office of Basic Energy Sciences.

3.7

\section{A SYSTEM FOR SIMULATING SHARED MEMORY IN HETEROGENEOUS DISTRIBUTED- MEMORY NETWORKS WITH SPECIALIZATION FOR ROBOTICS APPLICATIONS}

\author{
J. P. Jones A. L. Bangs \\ P. L. Butler*
}

(Abstract of paper presented at the 1992 IEEE International Conference on Robotics and Automation, Nice, France, May 12-14, 1992; Proc. pp. 2738-2744 (1992)]

Hetero Helix is a programming environment which simulates shared memory on a heterogeneous network of distributed-memory computers. The machines in the network may vary with respect to their native operating systems and internal representation of numbers. Hetero Helix presents a simple programming model to developers, and also considers the needs of designers, system integrators, and maintainers. The key software technology underlying Hetero Helix is the Use of a "compiler" which analyzes the data structure in shared memory and automatically generates code which translates data representations from the format native to each machine into a common format, and vice versa. The design of Hetero Helix was motivated in particular by the requirements of robotics applications. Hetero Helix has been used successfully in an integration effort involving $27 \mathrm{CPUs}$ in a heterogeneous network and a body of software totalling roughly 100,000 lines of code.

Research sponsored by U.S. DOE Office of Nuclears Energy, Office of Advanced Reactor Programs.

${ }^{*}$ Robotics and Process Systems Division. 
3.8

HETERO HELIX: SYNCHRONOUS

AND ASYNCHRONOUS CONTROL SYSTEMS IN HETEROGENEOUS DISTRIBUTED NETWORKS
J. P. Jones
P. L. Butler
S. E. Johnston ${ }^{\dagger}$
T. E. Heywood ${ }^{\ddagger}$

[Abstract of Robotics and Autonomous Systems 10(2-3). 85 (1992)]

Hetero Helix is a programming environment which simulates shared memory on a heterogeneous network of computers. The machines in the network can vary with respict to their native operating systems and internal representation of numbers Hetero Helix was designed to present a simple programming model to developers, and also considers the needs of designers, system integrators, and maintainers. The key software technology underlying Hetero Helix is the use of a "compiler" which analyzes the data structures in shared memory and automatically generates code which translates data representations from the format native to each machine into a format understood by all machines, and vice versa. This automatic code generation insures correctness and eases the burden on code developers, who would otherwise have to do the same job by hand. Hetero Helix has been used successfully in connection with an integration effort involving $27 \mathrm{CPU}$ s in a heterogeneous network and a body of software totalling roughly 100,000 lines of code.

Research sponsored by the Robotics for Advanced Reactors program of the Office of Nuclear Energy U.S. DOE.

* Robotics and Process Systems Division.

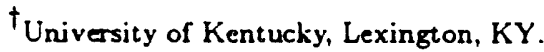

${ }^{\ddagger}$ University of Florida, Gainesville, FL.
3.9

\section{A CONCURRENT CONTROL ARCHITECTURE FOR AUTONOMOUS MOBILE ROBOTS USING ASYNCHRONOUS PRODUCTION SYSTEMS}

\author{
S. S. Iyengar* F. G. Piu \\ J. Graham* V. G. Hedge* \\ P. Graham*
}

[Abstract of Automation in Construction (in press)]

The quest for efficient real-time response by autonomous robots in hazardous environments necessitates not only fast computational schemes in expert systems but also requires insights on high performance data structures and concurrent algorithms that lead to elegant problem-solving methods in the AI discipline. Towards this objective this paper presents an Asynchronous Production System (APS) of architecture capable of monitoring and processing real-time information.

Research sponsored by U.S. DOE Office of Basic Energy Sciences.

*Louisiana State University, Baton Rouge, LA.

3.10

\section{DYNAMIC COUPLING EFFECTS IN MODELING AND CONTROL OF HARD CONTACT MOTION OF A MANIPULATOR}

\section{A. Unseren}

(Absiract of paper presented at the IEEE International Conference on Systems Engineering, Dayton, $\mathrm{OH}$, August 1-3, 1991; Proc. pp. 100-104 (1991)]

In the previous work on the hard contact mo tion of a single manipulator, only the kinematic coupling effects were taken into account. In this paper the dynamic coupling effects are considered in the modeling and control of an $\mathrm{N}$-joint, serial link manipulator turning a crank mechanism. By dynamically distributing the load, i.e., the crank, the generalized contact forces are decomposed into internal stress and motion components. A rigid body model is developed for the entire closedchain system. It is shown here that a subset of the internal stress contact forces may be explicitly controlled while simultaneovily and independently controlling the position of the system.

Research sponsored by U.S. DOE Engineering Research Program of the Office of Basic Energy Sciences. 
3.11

\section{INPUT RELEGATION CONTROL FOR GROSS MOTION OF A KINEMATICALLY REDUNDANT MANIPULATOR}

\section{A. Unseren}

(Abstract of ORNL/TM-12165, October 1992)

This report proposes a method for resolving the kinematic redundancy of a serial link manipulator moving in a three-dimensional workspace. The underspecified problem of solving for the joint velocities based on the classical kinematic velocity model is transformed into a well-specified problem. This is accomplished by augmenting the original model with additional equations which relate a new vector variable quantifying the redundant degrees of freedom (DOF) to the joint velocities. The resulting augmented system yields a well specified solution for the joint velocities. Methods for selecting the redundant DOF quantifying variable and the transformation matrix relating it to the joint velocities are presented so as to obtain a minimum Euclidean norm solution for the joint valocities. The approach is also applied to the problem of resolving the kinematic redundancy at the acceleration level. Upon resolving the kinematic redundancy, a rigid body dynamical model governing the gross motion of the manipulator is derived. A control architecture is suggested, which according to the model, decouples the Cartesian space DOF and the redundant DOF.

Research sponsored by U.S. DOE Office of Engineering Research Program, Basic Energy Sciences and Office of Technology Support Programs, Office of Nuclear Energy.
3.12

\section{AN IMPLEMENTATION OF REDUNDANCY RESOLUTION AND STABILITY MONITORING FOR A MATERIAL HANDLING VEHICLE}

\author{
A. L. Bangs F. G. Pin \\ S. M. Killough*
}

(Abstract of paper presented at the IEEE/IES Intelligent Vehicles '92, Detroit, MI, July 1-2, 1992)

The ATLAS (All-Terrain Lifter Articulated System) vehicle is a prototype for the nextgeneration Army material handling vehicle. The vehicle features a redundant manipulator with five degrees of freedom in a plane and a forklift endeffector. The original control system only allowed single joint motion control via a set of joysticks in the cab. A new controller was developed to automatically coordinate all joints in the system and allow the operator to directly control the motions of the end-effector with constant orientation via a single joystick. In addition, a stability monitor was developed which prevents the operator from tipping over the vehicle when manipulating heavy loads of uncertain weight, or warns him of approaching stability limits when driving. Finally, a load-compensation function was developed that automatically tips back the fork tines when a heavy load is lifted.

Research sponsored by U.S. DOE Engineering Research Program of the Office of Basic Energy Sciences and the U.S. Army Ft. Belvoir Research Development and Engineering Center.

* Robotics and Process Systems Division.

3.13

\section{AUTONOMOUS SURVEILLANCE FOR SURFACE RADIATION CONTAMINATION}

\author{
J. P. Jones \\ P. L. Butler* \\ M. Beckerman \\ F. J. Sweeney
}

[Abstracc of paper presented at the 1992 IEEE International Conference on Robotics and Automation, Nice, France, May 12-14, 1992; Proc. p. 63 (1992)]

There are more than one million 55-gallon drums in the U.S. storing low-level radioactive toxic waste. They must be monitored almost continuously to guard against leakage and the conse- 
quent environmental damage. Manual monitoring is a tedrous, repetitive, labor-intensive task. This video illustrates a proof-of-concept demonstration for the autonomous radiation surveillance of 55 . gallon drums.

The system consists of a number of components including ultrasonic sensing and interpretation, computer vision, parallel processing, range image analysis, control of a $7-D O F$ redundant manipulator, control of an omnidirectional mobile platform, obstacle avoidance, a graphical user interface, and sensor-based force-reflecting teleoperation. The computing environment is a relatively complex heterogeneous network, including a Silicon Graphics IRIS 4D/80GT, a DEC Microvax, several VME-based common-bus multiprocessors, an Apple Macintosh, and an IBM-PC/AT based NCUBE hypercube multicomputer.

Research sponsored by U.S. DOE Office of Nuclear Energy, Office of Advanced Reactor Programs.

* Robotics and Process Systems Division.

3.14

\section{DEMO 89 - THE INITIAL EXPERIMENT WITH THE HERMIES-III ROBOT}

\author{
D. B. Reister \\ J. P. Jones \\ P. L. Butler* \\ M. Beckerman \\ F. J. Sweeney
}

Abstract of paper presented at the 1991 IEEE Interna tional Conference on Robotics and Automation. Sacra mento, CA, April 7-12, 1991; Proc. Vol. 3, pp. 2562-2567 (April 1991)]

HERMIES-III is a large mobile robot designed for human scale experiments. The initial experiment with the robot (DEMO 89) was the cleanup of a simulated chemical spill. To perform the experiment, the robot was required to plan a path through an a prior known world, navigate along the path (avoiding unexpected obstacles) and locate and remove debris from a target area. This paper describes the software system that was developed to perform the experiment. The software system consisted of 19 processes that operated on a distributed set of heterogeneous computers.

Research sponsored by U.S. DOE Office of Nuclear Energy, Office of Technology Support Programs.

\footnotetext{
*Fuel Recycle Division.
}

3.15

\section{DESIGN OF AN OMNIDIRECTIONAL AND HOLONOMIC WHEELED PLATFORM PROTOTYPE}

\author{
S. M. Killough* F. G. Pin
}

(Abstract of paper presented at the 1992 IEEE International Conference on Robotics and Automation, Nice, France, May 12-14, 1992; Proc. pp. 84-90 (1992)]

This paper presents the concepts for a new family of wheeled platforms which feature full omnidirectionality with simultaneous and independent rotational and translational motion capabilities. We first describe the original "orthogonalwheels assembly" on which these platforms are based and discuss how a combination of these assemblies is used to generate an omnidirectional capability. The design and control of a prototype platform developed to test and demonstrate the proposed concepts is then described, and experimental results illustrating the full omnidirectionality of the platform with decoupled rotational and translational degrees of freedom are presented.

Research sponsored by U.S. DOE Engineering Research Program of the Office of Basic Energy Sciences.

${ }^{*}$ Robotics and Process Systems Division.

3.16

\section{MOBILE MANIPULATOR CONFIGURATION OPTIMIZATION USING EVOLUTIONARY PROGRAMMING}
J. R. McDonnell*
B. L. Andersen* W. C. Page*
F. G. Pin

[Abstract of paper presented at the First Annual Conference on Evolutionary Programming, La Jolla, CA, February 21-22, 1992; Proc. pp. 52-62 (1992)]

Multi-degree-of-freedom manipulators are becoming commonplace on mobile platforms. Full autonomy of mobile manipulator robotic systems will depend on the ability to resolve the inherent kinematic redundancy in task commutation. This work investigates the application of an evolutionary search strategy for determining near-optimal mobile manipulator configurations. Joint torques, obstacle avoidance and manipulability are incorporated in a multi-criteria optimization formulation. A variety of aspects of the evolutionary programming paradigm are addressed via empirical 
studies on a two degree-of-freedom (DOF) manipulator. These studies investigate full configuration vector versus partial configuration vector hutation as well as mutation strategies which incorporate cost and iteration number. The results of this study are then applied to a planar three DOF manipulator mounted on a single DOF mobile base. Experiments indicate that the configuration optimization problem is amenable to a variety of mutation strategies.

Research sponsored by U.S. DOE Engineering Research Program, Office of Basic Energy Sciences.

*NCCOSC/RDT\&E Division, San Diego, CA.

\subsection{7}

\section{A MODEL OF REACTIVE PLANNING FOR MULTIPLE MOBILE AGENTS}

\section{K. Fujimura}

[Abstract of paper presented at the 1991 IEEE Interna tional Conference on Robotics and Automation, Sacramento, CA, April 7-12, 1991; Proc. Vol. 2, pp. 1503-1509 (1991)]

Reactive planning is studied for multiple mobile agents. The approach taken is distributed, i.e., each planning agent independently plans its own action based on its map information. An environment contains mobile agents of different capacities regarding knowledge about the environment, planning algorithms, etc. A model for such reactive agents is described and simulation results are presented to show their behavior patterns. The work described in this paper is part of our continuing effort in studying multi-agent mobile robor systems.

Research sponsored by U.S. DOE Engineering Research Program of the Office of Basic Energy Sciences.

\subsection{8}

\section{MOTION PLANNING IN DYNAMIC ENVIRONMENTS}

\section{K. Fujimura}

[Abstract of book, Springer-Verlag, Tokyo (December 1991)]

Motion planning is an area in robotics that has received much attention recently. Much of the past research focuses on static environments - various methods have been developed and their characteristics have been well investigated. Although it is essential for advanced intelligent robots to be able to handle dynamic worlds, the problem of motion planning in dynamic domains is relatively little understood compared with static problems.

This research monograph is the first book dedicated to computational and algorithmic aspects of motion planning in dynamic environments. Chapter 2 contains a brief survey of motion planning problems and preliminaries. In Chapters 3 through 5, various properties of time-minimal motions are stated and proved which are of fundamental importance in the study of motion planning among dynamic obstacle. Chapter 6 is independent of the previous three chapters and treats the problem from a different perspective. Chapter 7 contains an investigation of a challenging problem of distributed mobile agents.

Research on the subject of the monograph has just begun and there are many important issues that are to be investigated. It is my hope that research contained in this monograph will serve as a basis for further studies and excite more research on related subjects.

Research sponsored by U.S. DOE Engineering Research Program of the Office of Basic Energy Sciences. 
3.19

\section{ON MOTION PLANNING AMIDST TRANSIENT OBSTACLES}

\section{K. Fujimura}

(Abstract of paper presented at the 1992 IEEE International Conference on Robotics and Automation, Nice. France, May 12-14, 1992; Proc. pp. 1488-1493 (1992)]

Motion planning in the presence of timedependent obstacles is studied. Much of the prior approaches assumes that the obstacles always exist in the environment whether they are stationary or in motion. In this paper, we consider the case that the environment contains obstacles whose existing periods are dependent on time, i.e., they may disappear and reappear in the environment. This formulation enables us to model a variety of time-varying situations that can arise in some application domains. For example, one can plan a motion in an environment where another agent rearranges the environment by picking up an object and placing it back at another location in the same environment. A concept similar to that of visibility is used to solve the problem. An algorithm is presented to generate a motion in such a dynamic domain and its time-minimality is proved. The algorithm runs in $O\left(n^{3} \log n\right)$ time, where $n$ is the total number of vertices in the environment.

Research sponsored by U.S. DOE Engineering Research Program of the Office of Basic Energy Sciences.

3.20

\section{NAVIGATION OF A CAR-LIKE MOBILE ROBOT USING A DECOMPOSITION OF THE ENVIRONMENT IN CONVEX CELLS}

\author{
H. A. Vasseur F. G. Pin \\ J. R. Taylor*
}

Abstract of paper presented at the 1991 IEEE Interna-
tional Conference on Robotics and Automation, Sacra-
mento, CA, April $7-12,1991$; Proc. Vol. 3, pp. 1496-1502 (1991)]

Due to their kinematics, car-like mobile robots cannot follow an arbitrary path. Besides obstacle avoidance, the path planning problem for such platforms has to satisfy two additional constraints: a lower bounded radius of turn, and a non holonomic constraint. When the robot is not circular, precise maneuvering always implies working in the configuration space of the vehicle. Due to the complexity of the problem, the planning of a path involves computer intensive methods, and rarely allows for real time applications.

However, in a convex polygonal cell, maneuvering can be completely liandled within geometric reasoning. In this simplified environment, joining two configurations may require maneuvers only at the beginning and at the end of the trajectory. The method consists of computing the possible maneuvers either starting from the initial configuration, or arriving at the final configuration. Just a few boundary configurations have to be checked to avoid collision. Maneuvers related to the initial configuration and to the final configuration can be connected by a straight trajectory because of the convexity of the cell, which allows precise maneuvering computation, without using the whole configuration space.

We describe a general environment by means of a graph connecting overlapping convex cells. To find a path between two configurations, the graph is searched in order to list the cells that have to be traversed. Then, intermediate configurations are computed inside the intersection of two adjacent cells. Finally, the trajectories generated inside each cell are assembled to produce global collision free paths in complex environments.

Research sponsored by U.S. DOE Engineering Research Program of the Office of Basic Energy Sciences.

${ }^{*}$ Central Engineering at $\mathrm{Y}-12$. 


\subsection{1 \\ CONTROL OF AUTONOMOUS MOBILE ROBOTS USING CUSTOM-DESIGNED QUALITATIVE REASONING VLSI CHIPS AND BOARDS}

\author{
F. G. Pin R. S. Pattay
}

(Abstract of paper presented at the Ninth Symposium on Energy Engineering Sciences, Argonne National Laboratory, Argonne, IL, May 13-15, 1991; Proc. 141-148 (1991)]

Two types of computer boards including custom-designed VLSI chips have been developed to provide a qualitative reasoning capability for the real-time control of autonomous mobile robots. The design and operation of these boards are described and an example of application of qualitative reasoning for the autonomous navigation of a mobile robot in a-priori unknown environments is presented. Results concerning consistency and modularity in the development of qualitative reasoning schemes as well as the general applicability of these techniques to robotic control domains are also discussed.

Research sponsored by U.S. DOE Engineering Research Program of the Office of Basic Energy Sciences.

\subsection{2}

\section{USING CUSTOM-DESIGNED VLSI FUZZY INFERENCING CHIPS FOR THE AUTONOMOUS NAVIGATION OF A MOBILE ROBOT}

\section{F. G. Pin \\ J. Symon ${ }^{*}$ \\ H. Watanabe* \\ R. S. Pattay}

[Abstract of paper presented at the 1992 IEEE International Conference on Intelligent Robots and Systems, Raleigh, NC, July 7-10, 1992; Proc. pp. 790-795 (1992)]

Two types of computer boards including custom-designed VLSI fuzzy inferencing chips have been developed to add a qualitative reasoning capability to the real-time control of autonomous mobile robots. The design and operation of these boards are first described and an example of their use for the autonomous navigation of a mobile robot is presented. The development of qualitative reasoning schemes emulating human-like navigation in a-priori unknown environments is discussed. An approach using superposition of elemental sensor-based behaviors is shown to allow easy development and testing of the inferencing rule base, while providing tor progressive addi- tion of behaviors to resolve situations of increasing complexity. The efficiency of such schemes, which can consist of as little as a dozen qualitative rules, is illustrated in experiments involving an autonomous mobile robot navigating on the basis of very sparse and inaccurate sensor data.

Research sponsored by U.S. DOE Engineering Research Program of the Office of Basic Energy Sciences.

* University of North Carolina at Chapel Hill, Chapel Hill, NC.

3.23

\section{MERGING THE FUZZY AND BEHAVIORIST APPROACHIS USING VLSI FUZZY INFERENCING CHIPS: AN EFFICIENT TECHNIQUE FOR THE AUTONOMOUS NAVIGATION OF A MOBILE ROBOT}
F. G. Pin
H. Watanabe*

[Abstract of book chapter in Industrial Applications of Fuzzy Control and Intelligent Systems, J. Yen and L. A. Zadeh, Eds., Van Nostrand Reinhold (in press)]

An approach merging the concepts of fuzzy logic and sensor-based behaviors has been developed to add a qualitative reasoning capability to the real-time control of autonomous mobile robots. The approach is implemented using custom-designed computer boards which include recently developed VLSI fuzzy inferencing chips. The design and operation of these boards are first described and an example of their use for the autonomous navigation of a mobile robot is presented. The development of qualitative reasoning schemes emulating human-like navigation in a-priori unknown environments is discussed. The approach using superposition of elemental sensorbased behaviors expressed in the Fuzzy Sets theoretic framework is shown to allow easy development and testing of the inferencing rule base, while providing for progressive addition of behaviors to resolve situations of increasing complexity. The efficiency of such schemes, which can consist of as little as a dozen qualitative rules, is illustrated in experiments involving an autonomous mobile robot navigating on the basis of very sparse and inaccurate sensor data.

Research sponsored by U.S. DOE Engineering Research Program of the Office of Basic Energy Sciences.

* University of North Carolina at Chapel Hill, Chapel Hill, iNC. 
3.24

\section{AUTONOMOUS NAVIGATION OF A MOBILE ROBOT USING CUSTOM- DESIGNED QUALITATIVE REASONING VLSI CHIPS AND BOARDS}
F. G. Pin
H. Watanabe*
J. Symon*
R. S. Pattay

[Abstract of paper presented at the 1992 IEEE International Conference on Robotics and Automation, Nice, France, May 12-14, 1992; Proc. 123-128 (1992)]

Two types of computer boards including custom-designed VLSI chips have been developed to add a qualitative reasoning capability to the real-time control of autonomous mobile robots. The design and operation of these boards are first described and an exarnple of their use for the autonomous navigation of a mobile robot is presented. The development of qualitative reasoning schemes emulating human-like navigation in a-priori unknown environments is discussed. The efficiency of such schemes, which can consist of as little as a dozen qualitative rules, is illustrated in experiments involving an autonomous mobile robot navigating on the basis of very sparse and inaccurate sensor data.

Research sponsored by U.S. DOE Engineering Research Program of the Office of Basic Energy Sciences.

*University of North Carolina at Chapel Hill.

\subsection{5}

\section{SENSOR-BASED DRIVING OF A CAR WITH FUZZY INFERENCING VLSI CHIPS AND BOARDS}

\section{F. G. Pin Y. Watanabe}

[Abstract of paper presented at the 4th International Symposium on Robotics and Manufacturing, Santa Fe, NM, November 11-13, 1992; Proc. Vol. 4, pp. 613-619 (1992)]

This paper discusses the sensor-based driving of a car in a-priori unknown environments using "human-like" reasoning schemes. The schemes are implemented on custom-designed VLSI fuzzy inferencing boards and are used to investigate two control modes for driving a car on the basis of very sparse and imprecise range data. In the first mode, the car navigates fully autonomously, while in the second mode, the system acts as a driver's aid providing the driver with linguistic (fuzzy) commands to turn left or right and speed up, slow down, stop, or back up depending on the obstacles perceived by the sensors. Experiments with both modes of control are described in which the system uses only three acoustic range (sonar) sensor channels to perceive the environment. Sample results are presented which illustrate the feasibility of developing autonomous navigation systems and robust safety enhancing driver's aid using the new fuzzy inferencing VLSI hardware and "human-like" reasoning schemes.

Research sponsored by U.S. DOE Engineering Research Program of the Office of Basic Energy Sciences.

*University of North Carolina at Chapel Hill, Chapel Hill, NC.

\subsection{6}

\section{IMPLEMENTING VALIANT'S LEARNABILITY THEORY USING RANDOM SETS}

\section{E. M. Oblow}

[Abstract of Machine Learning 8, 45 (1992)]

A general learning framework which uses random sets is introduced for solving discretespace classification problems. This framework is based on the pac-learning formalism introduced by Valiant (1984) and generalized in set-theoretic terms by Blumer, et al., (1989). The random set version of this theory is used to develop an algorithm which is a particularly efficient search scheme. This is accomplished by recasting the representational class and constructive proof presented in Valiant (1984) into random set terms and implementing it as an exhaustive search algorithm. The algorithm is a problem-specific incremental (psi) approach in that it satisfies learnability criteria for distribution-specific problems as examples are being sampled. Some theoretical and empirical analyses are presented to demonstrate the convergent pac-learnability and sample complexity of this psi-algorithm. Its performance is then tested on the multiplexor class of problems. This class has been analyzed by others as a benchmark for decision trees and genetic classifiers. Results from these test cases show that, despite using 
an exhaustive search, this random set implementation is computationally competitive with these more established methods (which use empirically proven heuristics). Conclusions are drawn about potential further improvements in the efficiency of this approach.

Research sponsored by U.S. DOE Office of Basic Energy Sciences.

\section{N-LEARNERS PROBLEM: LEARNING BOOLEAN COMBINATION OF HALFSPACES}

\author{
N. S. V. Rao* E. M. Oblow \\ C. W. Glover
}

(Abstract of ORNL/TM-11905, March 1991)

Given two subsets $S_{1}$ and $S_{2}$ (not necessarily finite) of $\Re^{d}$ separable by a Boolean combination of $N$ halfspaces, we consider the problem of learning (in the sense of Valiant) the separation function from a finite set of examples, i.e. we produce with high probability a function close the actual separating function. Our solution consists of a system of $N$ perceptrons and a single consolidator which combines the outputs of the individual perceptrons. We show that an off-line version of this problem, where the examples are given in a batch, can be solved in time polynomial in the number of examples. We also provide an on-line learning algorithm that incrementally solves the problem by suitably training a system of $N$ perceptrons much in the spirit of the classical perceptron learning algorithm. This solution constitutes an example of a composite system of $N$ learners capable of accomplishing a task that is not achievable by a single learner, for a single perceptron is incapable of separating sets that are not linearly separable.

Research sponsored by U.S. DOE Office of Engineering Research Program of the Office of Basic Energy Sciences and by the Office of Naval Research Intelligent Systems Program, U.S. Department of Defense.

*Old Dominion University, Norfolk, VA.

\subsection{8}

\section{N-LEARNERS PROBLEM: FUSION OF CONCEPTS}
N. S. V. Rao*
E. M. Oblow
C. W. Glover
G. E. Liepins

(Abstract of ORNL/TM-11904, September 1991)

We are given $N$ learners each capable of learning concepts (subsets) of a domain set $X$ in the sense of Valiant, i.e. for any $c \in C \subseteq 2^{X}$, given a finite set of examples of the form $<x_{1}, M_{c}\left(x_{1}\right)$ $>_{;}<x_{2}, M_{c}\left(x_{2}\right)>; \ldots ;<x_{l}, M_{c}\left(x_{l}\right)>$ generated according to an unknown probability distribution $P_{X}$ on $X$, each learner produces a close approximation to $c$ with a high probability. We are interested in combining the $N$ learners using a single fuser or consolidator. We consider the paradigm of passive fusion, where each learner is first trained with the sample without the influence of the consolidator. The composite system is constituted by the fuser and the individual learners. We consider two cases: open and closed fusion. In open fusion the fuser is given the sample and the hypotheses of the individual learners; we show that the fusion rule can be obtained by formulating this problem as another learning problem. For the case all individual learners are trained with the same sample, we show sufficiency conditions that ensure the composite system to be better than the best of the individual: the hypothesis space of the consolidator (a) satisfies the isolation property of degree at least $N$, and (b) has Vapnik-Chervonenkis dimension less than or equal to that of every individual learner. If individual learners are trained by independently generated samples, we obtain a much weaker bound on the VC-dimension of the hypothesis space of the fuser. Second, in closed fusion the fuser does not have an access to either the training sample of the hypotheses of the individual learners. By suitably designing a linear threshold function of the outputs of individual learners, we show that the composite system can be made better than the best of the learners.

Research sponsored by U.S. DOE Engineering Research Program of the Office of Basic Energy Sciences and by the Office of Naval Research Intelligent Systems Program, U.S. Department of Defense.

*Old Dominion University, Norfolk, VA. 
3.29

\section{ANALYSIS OF COMPLEXITY BOUNDS FOR PAC-LEARNING WITH RANDOM SETS}

\author{
E. M. Oblow V. R. R. Uppuluri
}

(Abstract of ORNL/TM-11844, August 1991)

Learnability in Valiant's pac-learning formalism is reformulated in terms of expected (average) error instead of confidence and error parameters. A finite-domain, random set formalism is introduced to develop algorithm-dependent, distribution-specific analytic error estimates. Two random set theorems for finite concept-spaces are presented to facilitate these developments. Analyses are carried out for several illustrative problems with worst-case and semi-uniform distributions of learning examples. Analytic bounds on the sample size needed to achieve a specified average error are established. Useful approximations for these bounds and a worst-case distribution are also derived. Conclusions are drawn about the potential value of average-error bounds in improving the stated efficiency of pac-learning algorithms.

Research sponsored by U.S. DOE Office of Basic Energy Sciences and by Office of Naval Research U.S. Department of Defense.

\subsection{0}

\section{ALGORITHMS FOR RECOGNIZING PLANAR POLYGONAL CONFIGURATIONS USING PERSPECTIVE IMAGES}

\author{
N. S. V. Rao* W. Wu* \\ C. W. Glover
}

[Abstract of IEEE Transactions on Robotics and Automation 8(4), $480(1992)]$

We consider a simplified abstraction of the problem of recognizing planar arrangements of objects using camera pictures taken from unknown positions. A set of polygons in plane is called a planar polygonal configuration. Given perspective images $P$ and $Q$ corresponding to planar polygonal configurations, the matching problem is to determine if $P$ and $Q$ correspond to the same configuration. We present an optimal $\theta(n \log n)$ time algorithm to solve this problem, where $n$ is the total number of vertices of polygons in each image. Our algorithm is obtained by combining the ideas of cross ratios, which are well known to be invariant under perspective projections, and the first fundamental theorem of perspective projections. This algorithm has been implemented and tested.

Research sponsored by U.S. Department of Energy.

*Old Dominion University, Norfolk, VA.

\subsection{1}

\section{EMPIRICAL CHARACTERIZATION OF A HIGH INTENSITY LED PROXIMITY SENSOR}

\section{J. E. Baker}

[Abstract of Sensors (in press); also ORNL/TM-11972, November 1991]

Many robotic operations require accurate and precise distance measurements to arbitrary targets in an unstructured environment. Such information can be used in path planning, object avoidance, surface following, topological mapping, relative positioning, artifact resolution, etc. Hence, there is a significant need for a non-contact proximity sensor system which is minimally effected by target composition, orientation, color, texture, etc. and to empirically understand its behavior with respect to such target characteristics. This paper describes a most promising sensing technology (high intensity LED triangulation) and presents an empirical analysis of a representative, commercial system.

Research sponsored by U.S. DOE Office of Technology Development. 


\section{TERRAIN FOLLOWING OF ARBITRARY SURFACES USING A HIGH INTENSITY LED PROXIMITY SENSOR}

\author{
J. E. Baker
}

(Abstract of ORNL/TM-11991, January 1992)

Many robotic operations, e.g., mapping, scanning, feature following, etc., require accurate surface following of arbitrary targets. This paper presents a versatile surface following and mapping system designed to promote hardware, software and application independence, modular development, and upward expandability. These goals are met by: a full, a priori specification of the hardware and software interfaces; a modular system architecture; and a hierarchical surface-data analysis method, permitting application specific tuning at each conceptual level of topological abstraction. This surface following system was fully designed and implemented independently of any specific robotic host, then successfully integrated with and demonstrated on a completely a priori unknown, real-time robotic system.

Research sponsored by U.S. DOE Office of Technology Development.

\subsection{3}

\section{PHASE I REPORT ON SENSOR TECHNOLOGY, DATA FUSION AND DATA INTERPRETATION FOR SITE CHARACTERIZATION}

\section{Beckerman}

(Abstract of ORNL/TM-11971, October 1991)

In this report we discuss sensor technology, data fusion and data interpretation approaches of possible maximal usefulness for subsurface imaging and characterization of land-fill waste sites. Two sensor technologies, terrain conductivity using electromagnetic induction and ground penetrating radar, are described and the literature on the subject is reviewed. We identify the maximum entropy stochastic method as one providing a rigorously justifiable framework for fusing the sensor data, briefly summarize work done by us in this area, and examine some of the outstanding issues with regard to data fusion and interpretation.

Research sponsored by U.S. DOE Office of Technology Development.

\section{LASER RANGE CAMERA CALIBRATION}

\author{
K. Storjohann* E. Saltzen ${ }^{\dagger}$
}

(Abstract of ORNL/TM-11723, July 1991)

This paper describes an imaging model that was derived for use with a laser range camera (LRC) developed by the Advanced Intelligent Machines Division of Odetics. However, this model could be applied to any comparable imaging system. Both the derivation of the model and the determination of the LRC's intrinsic parameters are explained. For the purpose of evaluating the LRC's external orientation (extrinsic parameters), a transformation of the LRC's imaging model into a standard camera's (SC) pinhole model is derived. By virtue of this transformation, the evaluation of the LRC's external orientation can be found by applying any SC calibration technique. Experimental results prove the accuracy of the derived camera model as well as the proposed calibration procedure.

Research sponsored by U.S. DOE Office of Basic Energy Sciences.

*Ruhr Universitat Bochum, West Germany.

$\dagger^{\dagger}$ California State Unirersity, Turlock, CA.

\section{RESTORATION AND FUSION OF LASER RANGE CAMERA IMAGES}

\author{
M. Beckerman \\ F. J. Sweeney
}

[Abstract of paper presented at the 1992 SPIE Conference on Neural and Stochastic Methods in Image and Signal Processing, San Diego, CA, July 20-23, 1992; Proc. Vol. 1766, pp. 454-465 (1992)]

In this work we introduce Markov cross entropic prior in the Bayesian restoration and fusion of laser range images. These cross entropic priors are used to model smoothness of the surfaces and linearity of the discontinuities. The priors are defined over a pair of coupled Markov random fields representing the corresponding pixel and line processes. Gibbsian maximum a posteriori estimates are then found using simulated annealing. Range image data are discussed, and results are presented for synthetic range images.

Research sponsored by U.S. DOE Office of Basic Energy Sciences and Nuclear Energy Office. 
3.36

\section{SENSOR DATA FUSION FOR MOBILE ROBOTS}

\section{Beckerman}

[Abstract of paper presented at the Institute for Mathematical Statistics Special Topics Meeting on Industrial Statistics, Philadelphia, PA, June 10-12, 1991; Proc. IMS Bulletin, Vol. 20, pp. 162-163 (1991)]

One of the research goals at Oak Ridge $\mathrm{Na}$ tional Laboratory's Center for Engineering Systems Advanced Research is the development of autonomous mobile robots which are capable of surveillance, navigation, and manipulation in environments which are unstructured to an appreciable extent. These robots are equipped with a variety of sensors including CCD and laser range cameras. In this paper we discuss the sensory data as acquired by the robots. We will examine the distributions of the geometric properties of the intensity edges and surface regions of typical scenes, and decompose the errors in these quantities into their systematic and random components. Sensor data fusion is a strategy for reducing errors and uncertainties. We will review the techniques we have developed for fusing data from the cameras. We will then discuss statistical methods which have been developed for combining information, and explore some of the underlying issues.

Research sponsored by U.S. DOE Engineering Research Program of the Office of Basic Energy Sciences and by the Office of Nuclear Energy, Office of Technology Support Programs.

\subsection{7}

\section{A BAYES-MAXIMUM ENTROPY METHOD FOR MULTI-SENSOR DATA FUSION}

\section{Beckerman}

[Abstract of paper presented at the 1992 IEEE International Conference on Robotics and Automation, Nice, France, May 12-14, 1992; Proc. pp. 1668-1674 (1992)]

In this paper we introduce a Bayes-Maximum Entropy formalism for multi-sensor data fusion, and present an application of this methodology to the fusion of ultrasound and visual sensor data as acquired by a mobile robot. In our approach the principle of maximum entropy is applied to the construction of priors and likelihoods from the data. Distances between ultrasound and visual points of interest in a dual representation are used to define Gibbs likelihood distributions. Both one- and two-dimensional likelihoods are presented, and cast into a form which makes explicit their dependence upon the mean. The Bayesian posterior distributions are used to test a null hypothesis, and Maximum Entropy Maps used for navigation are updated using the resulting information from the dual representation.

Research sponsored by U.S. DOE Engineering Research Program of the Office of Basic Energy Sciences, and by the Office of Nuclear Energy, Office of Technology Support Programs.

\subsection{8}

\section{CLASSIFIER SYSTEM LEARNING OF BOOLEAN CONCEPTS}

\author{
G. E. Liepins $\quad$ L. W. Wang*
}

[Abstract of paper presentea at the Fourth International Conference on Genetic Algorithm, San Diego, CA, July 13-16, 1991; Proc. Belew and Booker, Eds., Morgan Kauf mann Publishen (1991)]

We investigate classifier system learning of Boolean concepts. We introduce a symmetric reward-penalty mechanism, speciation, generality thresholds and rule evaluation by queries. These enable the classifier system to learn the 20 multiplexor significantly faster than previously reported for classifier systems. Conversely, we provide theoretical analyses that suggest that classifier systems are not competitive with the best known learning algorithms for stationary deterministic Boolean problems. We suggest instead that they are particularly well suited to non-stationary problems for which the target concept evolves over time.

Research sponsored by U.S. Department of Energy.

*University of Tennessee, Knoxville, TN. 


\subsection{9}

\section{POLYNOMIALS, BASIS SETS, AND DECEPTIVENESS IN GENETIC ALGORITHMS}

\author{
G. E. Liepins M. D. Vose* \\ [Abstract of Complex Systems 5, 45 (1991)]
}

The degree to which the genetic optimization process is transparent is in part determined by the form of the objective function. We develop two forms from first principles; polynomial forms and basis sets. We characterize three function classes that are fully easy for the genetic algorithm in terms of the polynomial representation. We generate functions of varying degrees of deceptiveness in terms of the representation provided by basis sets. We further show the relationship between these representations and the more standard Walsh polynomial.

Research sponsored by Director's Discretionary Fund.

* University of Tennessee, Knoxville, TN.

\subsection{0}

\section{GENETIC ALGORITHMS AND COMPUTERIZED TRADING STRATEGIES}

\section{R. J. Bauer, Jr. ${ }^{*} \quad$ G. E. Liepins}

(Abstract of book chapter in Expert Systems in Finance, pp. 89-100, Elsevier Science Publishers, North Holland (1992)]

Modern computers have provided investment firms with the capability of quickly analyzing numerous alternative trading strategies. However, even computers are severely limited in terms of exhaustive search. When portfolios are examined in a multi-period context, the number of strategy combinations can grow, for practical purposes, without bound. For this reason, various models and algorithms have been used to make the computational problems manageable.

This article describes a relatively new class of algorithms that are being used to tackle problems not amenable to other computational techniques. An example concerning asset allocation strategies illustrates the power of the algorithm, and suggests other applications; the algorithms seem to be well-suited for analyzing certain types of market timing and tactical asset allocation decisions.

Research sponsored by U.S. Department of Energy.

*St. Mary's University, San Antonio, TX.

\subsection{1}

\section{SCHEMATA DISRUPTION}

\section{D. Vose $\quad$ G. E. Liepins}

(Abstract of paper presented at the Fourth International Conference on Genetic Algorithms, San Diego, CA, July 13-16, 1991; Proc. Belew and Booker, Eds., Morgan Kaufmann Publishers (1991)]

We use results of Battle, Liepins, and Vose to motivate a reexamination of schemata disruption. We formalize a "building block hypothesis" in which the relation of building blocks to utilities is not prominent. What does matter is how the crossover operator interacts with schemata. We argue that this interaction determines what the building blocks are, and once determined, these building blocks are the appropriate objects on which to conduct schemata analysis.

Research sponsored by U.S. Department of Energy and Directors' Discretionary Fund.

*University of Tennessee, Knoxville, TN. 


\section{HUMAN FACTORS}

3.42

\section{A RESEARCH PROFILE OF THE COGNITIVE SYSTEMS AND HUMAN FACTORS GROUP AT THE OAK RIDGE NATIONAL LABORATORY}

\author{
H. E. Knee R. J. Carter
}

[Abstract of paper presented at the Human Factors Society Annual Meeting, San Francisco, CA, September 2-6, 1991; Proc. Vol. 2, pp. 1175-1179 (1991)]

After World War II, a system of national laboratories was created to foster a suitable environment for scientific research. Today, human factors activities are in evidence at most of the nine U.S. Department of Energy (DOE) multi-program national laboratories. This paper, which focuses on the Cognitive Systems and Human Factors Group at the Oak Ridge National Laboratory, is one of nine contributions made by various DOE laboratories in discussing human factors research at the various facilities. A brief history, primary areas of research and future directions are included.

Research sponsored by U.S. Department of Ene:gy.

\subsection{3}

\section{INDIVIDUAL AND TEAM COMMUNICATION IN A DYNAMIC TASK}
M. Terranova
D. Hartley*
B. G. Coury ${ }^{\dagger}$
K. N. Hooper ${ }^{\dagger}$

(Abstract of paper presented at the 35th Annual Human Factors Society Meeting, San Francisco, CA, September 13-16, 1991)

Communication is a fundamental component of complex systems where people work together to make decisions and solve problems. Unfortunately, most research concerned with decision making and problem solving with complex systems has focused on the behavior and performance of individuals rather than crews, teams or groups of people. The purpose of this paper is to report the results of an experiment that has focused on the differences between individual and team per. formance in a process control environment using graphic and alphanumeric display representations of system. Its emphasis is on communication. The role of communication in team performance and its affect on a person's mental model was explored in this research by using a combination of performance measures and verbal protocol analysis. The content of the operators' communication was analyzed in terms of the type and frequency of communication.

Research sponsored by U.S. Department of Energy.

*University of Tennessee, Knoxville, TN.

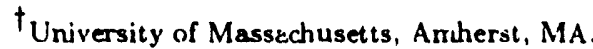

3.44

\section{SURVEY OF HUMAN FACTORS APPLICATIONS OF ARTIFICIAL NEURAL NETWORKS}

\author{
P. F. Spelt \\ [Abatract of Human Factors Society Bulletin 35(3), 4 \\ (1992)]
}

An introduction to artificial neural networks (ANNs) was presented in the July HFS Bulletin, in which a brief section described some possible uses of ANNs in the field of human factors. The present article summarizes papers presented at the Second Workshop on Neural Networks at Auburn University, February 11-13, 1991. The ANN/human factors section of the workshop was generated from responses to an electronic bulletin board posting in early September 1990. The posting requested a reply from those who were using ANNs in some human factors context, and received responses from around the world: one response was received from a researcher in England; another from Japan, and numerous replies from North America. All those doing ANN work who responded were included in the workshop sessions except for the one from England, who was unable to attend. Thus, it seems appropriate to conclude that the work presented here is representative of the current "state of the art" of ANN applications in human factors work. The articles summarized here range from a general theoretical analysis of the use of ANNs, to both theoretical and experimental applications of ANNs 
to human factors problems. Three of the articles use $\mathrm{ANNs}$ in the traditional pattern-recognition function; another article proposes to use an ANN as a model of human perception for skilled decision making. It appears that serious work exploring the functioning of ANNs in the human factors field is under way, with the promise of real benefits to come in the future.

Research sponsored by U.S Department of Energy.

\subsection{5}

\section{INTRODUCTION TO ARTIFICIAL NEURAL NETWORKS FOR HUMAN FACTORS}

\author{
P. F. Spelt \\ [Abstract of Human Factors Society Bulletin 34(7), 1 \\ (1991)]
}

Over the past ten or fifteen years, there has been increasing interest in artificial neural networks (ANNs), a "new" computing technology in the field of artificial intelligence, which actually dates back to 1943 , when McCulloch and Pitts published a mathematical simulation of nervous system activity. More recently, controversy, fervor, and adamant statements, pro and con, have surrounded this technology, both in print and in person at workshops and conferences. This article presents a summary introduction to ANNs, at the end of which will be a brief survey of uses of ANNs in human factors work. A second article, to appear in the Bulletin in September, will provide a survey of uses of ANNs in the field of human factors, based on papers presented at the 2nd Workshop on ANNs at Auburn University in February, 1991.

Research sponsored by U.S. DOE Office of Basic Energy Sciences.
3.46

\section{HUMAN FACTORS AND SAFETY ISSUES ASSOCIATED WITH ACTINIDE RETRIEVAL FROIM SPENT LIGHT WATER REACTOR FUEL ASSEMBLIES}

\author{
P. F. Spelt
}

Abstract of paper presented at the 36th Annual Human Factors Society Meeting, Atlanta, GA, October 12-16, 1992; Proc. Pp. 1158-1162 (1992)]

A major problem in environmental restoration and waste management is the disposition of used fuel assemblies from the many light water reactors in the United States, which present a radiation hazard to those whose job is to dispose of them, with a similar threat to the general environment associated with long-term storage in fuel repositories around the country. Actinides resident in the fuel pins as a result of their use in reactor cores constitute a significant component of this hazard. Recently, the Department of Energy has initiated an Actinide Recycle Program to study the feasibility of using pyrochemical (molten salt) processes to recover actinides from the spent fuel assemblies of commercial reactors. This project concerns the application of robotics technology to the operation and maintenance functions of a plant whose objective is to recover actinides from spent fuel assemblies, and to dispose of the resulting hardware and chemical components from this process. Such a procedure involves a number of safety and human factors issues. The purpose of the project is to explore the use of robotics and artificial intelligence to facilitate accomplishment of the program goals while maintaining the safety of the humans doing the work and the integrity of the environment. This project will result in a graphic simulation on a Silicon Graphics workstation as a proof of principle demonstration of the feasibility of using robotics along with an intelligent operator interface. A major component of the operator-system interface is a hybrid artificial intelligence system developed at Oak Ridge National Laboratory, which combines artificial neural networks and an expert system into a hybrid, self-improving computer-based system interface.

Research sponsored by U.S. Department of Energy. 
3.47

\section{ADVANCED CONTROL ROOM DESIGN REVIEW GUIDELINES: MERGING OLD AND NEW}
R. J. Carter
J. A. Wachtel ${ }^{*}$

Abstract of paper presented at the 36th Anmual Human Factors Meeting, Atlanta, GA, October 12-16, 1992; Proc. pp. 423-427 (1992)]

The nuclear power industry is currently developing operator interface systems based on innovative applications of digital computers. To assure that this advanced technology is inciorporated in a way that maximizes the potential safoty benefits of the technology and minimizes the potential negative effects on human performance, human factors principles must be considered. NUREG-0700 contains guidelines for the review of operator interfaces. However, in ligat of the rapid technological advances in digital techuology which have taken place in the eleven years since its publication, it is no longer adear ase to assess ihe rapidly changing Luman-system interfaces. A research program, the purpose of which is to upgrade NUREG-0700, has teen initiaced. Thus far a set of draft advanced control room design review (ACRDR) guidelines has been compiled. Thre tasks, which were oriented toxards integrating the applicable guidelines in NUREG-0700 inco the ACRDR document, are described in the paper.

Research sponsored by U.S. DOE and by the L'.S. Nuclear Regulatory Commissiou, Ofice of Nuclear Regulatory Re search.

*U.S. Nuclear Regulatory Commission, Rockvilie, MD.

\subsection{8}

\section{MODELS OF HUMAN OPERATORS: THEIR NEED AND USEFULNESS FOR IMPROVEMENT OF ADVANCED CONTROL SYSTEMS AND CONTROL ROOMS}

\section{H. E. Knee J. C. Schryver}

\footnotetext{
(Abstract of paper presented at the International Conference on Fast Reactors and Related Fuel Cycles, Kyoto Japan. October 28-November 1, 1991; Proe. Vol. 1 pp. 4.5-1 - 4.5-10 (1991)]

Models of human behavior and cognition (HB\&C) are necessary for understanding the total response of complex systems. Many such.
}

models have come available over the past thirty years for various applications. Many potential model users remain skeptical about their practicality, acceptability, and usefulness. Such hesitancy stems in part from disbelief in the ability to model complex cognitive r rocesses, and a belief that relevant human behavior can be adequately accounted for through the use of common-sense heuristics. This paper will highlight several models of HB\&C and identify existing and potential applications in attempt to dispel such notions.

Research sponsored by U.S. DOE Office of Technology Support Programs.

\subsection{9}

\section{A TRAINING EFFECTIVENESS PROGRAM FOR TRAINING SYSTEMS}

\section{R. J. Carter}

(Abstract o. paper presented at the Operations Research Society of America Conference, Nashville, TN, May 5-8, 1991)

Most training systems are delivered to the customer without the developer being required to prove that they are really training effective. Many systems, unfortunately, are developed under the false assumption that they will provide effective training. A training effectiveness program must become part of the training developer's design program in order to ensure that the iraining system is indeed training effective.

A training effectiveness rrogram methodology will be described. It consists of training effectiveness tasks which should be performed, analytical methodologies which should be employed, and training effectiveness tests and evaluation whirh should be implemented.

Research sponsored by U.S. Department of Inergy. 
3.50

\section{ON THE DESIGN AND \\ DEVELOPMENT OF A HUMAN-ROBOT SYNERGISTIC SYSTEM}

\author{
F. G. Pin L. E. Parker \\ F. Depiero ${ }^{\dagger}$
}

[Abstract of Robotics and Autonomous Systems 10(2), 161 (1992)]

A great variety of robotic systems have been developed over the past decades, varying from fully human-controlled teleoperated systems, to fully motion-programmed, industrial-type devices, to sensor-equipped "intelligent" machines with autonomous perception and reasoning capabilities. All these systems, however, have very static roles in supporting humans, in the sense that their capabilities are limited to very narrow functions or task domains and they cannot really adapt to changing environments where entirely new tasks may be necessary. By considering these systems as part of overall human-machine systems, interest- ing adaptation capabilities can be envisioned for the machine through the sharing of control and experiences, and observation of the human actions. This paper proposes a general conceptual architecture for enhancing the synergy between humans and machines in such cooperative systems, and investigates an implementation of the concept to the synergistic integration of teleoperative and autonomous operation of a manipulator arm for the execution of sequential tasks. The proposed architecture reflects a unique blend of many disciplines of artificial or machine intelligence and the functioning of its major modules is discussed. The paper then focuses on initial implementations of the Job Planner, Task Allocator, and Execution Monitor modules to discuss simple proof-of-principle experiments illustrating the coalescence of these modules needed to achieve skill-based dynamic task planning, allocation, and execution.

Resenrch sponsored by U.S. Army, Advanced Concept Technology Program.

* On leave o: absenre.

$\dagger^{\dagger}$ Fuel Recycle Division.

\section{INFORMATICS}

\subsection{1}

\section{GRAIL: AN INTEGRATED ARTIFICIAL INTELLIGENCE SYSTEM FOR GENE RECOGNITION AND INTERPRETATION}

\author{
X. Guan R. J. Mural \\ J. R. Einstein R. C. Mann \\ E. C. Uberbacher
}

[Abatract of paper presented at the Eighth IEEE Conference on Artificial Intelligence for Applications, Monterey, CA, March 2-6, 1992; Proc. pp. 9-13 (1992)]

This paper describes the development of an integrated Artificial Intelligence system GRAIL (Gene Recognition and Analysis Internet Link). This system uses a combinaticn of multisensor/neural network, expert system, and parallel search tools to recognize and interpret genes in DNA sequences. A simple electronic mail (E-mail) interface makes the system accessible through Internet. The strength of our system in recognizing and interpreting genes in DNA sequences and the simple E-mail interface have already attracted more than 150 users. The successful integration of neural network, expert system and highly parallel search tools to solve a problem as complicated as gene interpretation indicates that the technology itself is likely to have many applications in solving other problems.

Research sponsored by U.S. DOE Office of Health and Envirommental Research.

* Biology Division. 
3.52

\section{AN ARTIFICIAL INTELLIGENCE APPROACH TO DNA SEQUENCE FEATURE RECOGNITION}

\author{
R. J. Mural* \\ J. R. Einstein \\ X. Guan \\ R. C. Mann \\ E. C. Uberbacher
}

[Abstract of Trends in Biotechnology 10, 66 (1992)]

The ultimate goal of the Human Genome project is to extract the biologically relevant information recorded in the estimated 100,000 genes encoded by the $3 \times 10^{9}$ bases of the human genome. This necessitates development of reliable computer-based methods capable of analyzing and correctly identifying genes in the vast amounts of DNA-sequence data generated. Such tools may save time and labor by reducing the need for, for example, lengthy screening of cDNA libraries. They may also facilitate the localization of human disease genes by identifying candidate genes in prom sing regions of anonymous DNA sequence.

Research sponsored by U.S. DOE Office of Health and Er vironmental Research.

* Biology Division.

\subsection{3}

\section{GAP - A COMPUTER PROGRAM FOR GENE ASSEMBLY}

\author{
J. R. Einstein \\ E. C. Uberbacher \\ $X$. Guan \\ R. J. Mural*
}

R. C. Mann

(Abstrac: of ORNL/TM-11924, September 1991)

A computer program, GAP (Gene Assembly Program), has been written to assemble and score hypothetical genes, given a DNA sequence containing the gene, and the outputs of several other programs which analyze the sequence. These programs include the coding-recognition and splicejunction-recognition modules developed in this laboratory. GAP is a prototype of a planned system in which it will be integrated with an expert system and rule base. Initial tests of GAP have been carried out with four sequences, the exons of which have been determined by biochemical methods. The highest-scoring hypothetical genes for each of the four sequences had percent correct splice junctions ranging from 50 to $100 \%$ (average $81 \%$ ) and percent correct bases ranging from 92 to $100 \%$ (average $96 \%$ ).

Research sponsored by U.S. DOE Office of Health and Environmental Research and the Laboratory Directed Research and Development Programs.

*Biology Division.

$\mathbf{3 . 5 4}$

\section{GENE RECOGNITION AND ASSEMBLY IN THE GRAIL SYSTEM: PROGRESS AND CHALLENGES}

\author{
E. C. Uberbacher \\ J. R. Einstein
X. Guan
R. J. Mural

(Abstract of paper presented at the Second International Conference on Bioinformatics, Supercomputing, and Complex Genome Analysis, St. Petersburg Beach, FL, June 4-7, 1992)

GRAIL is a comprehensive system being constructed to analyze and characterize the genetic structure of DNA sequences. A number of program modules supply information to the system including the Coding Recognition Module (CRM), which forms the basis of the current e-mail GRAIL server system. Additional modules determine the positions and scores of possible splice junctions, the positions of potential translation-initiation sites, the coding strand for each gene, and the probable-translation-frame function over the sequence. The Gene Assembly Program module (GAP) attempts to predict the sequence of the spliced mRNA for a gene from the genomic DNA sequence. It constructs and scores gene modules, given a DNA sequence and the outputs of the other GRAlL modules for the sequence. GAP tests combinations of those splice junctions which are within acceptable distance from the initial predicted edges of the coding regions. Every complete gene model, comprising translation-initiation site, splice junctions and stop codon, which agrees with GAP's set of rules is scored, and the ten highestscoring models are saved. Each gene model's score depends on the input scores of splice junctions used in the model, their positions relative to the initial predicted edges of the included coding regions, and the degree of agreement of the entire model with the probable-translation-frame func- 
tion. If error conditions are detected, the present version of GAP attempts to correct them by the insertion and/or deletion of one or more coding regions. These insertions and deletions have resulted in a net improvement of gene models, and a particularly large improvement in the recognition and characterization of very short coding regions. The results of GRAIL including the GAP module for 26 sequences from GenBank, each with an experimentally characterized gene, are quite promising and demonstrate the feasibility of constructing largely accurate gene models strictly on the basis of DNA sequence data.

Research sponsored by U.S. DOE Office of Health and Environmental Research.

*Biology Division.

\subsection{5}

\section{COMPUTER-BASED CONSTRUCTION OF GENE MODELS USING THE GRAIL GENE ASSEMBLY PROGRAM}

\section{J. R. Einstein \\ R. J. Mural ${ }^{*}$ \\ X. Guan \\ E. C. Uberbacher}

(Abstract of ORNL/TM-12174, September 1992)

The Gene Assembly Program (GAP), a module of GRAIL, assembles and scores gene models, given a DNA sequence and the outputs of other GRAIL modules for the sequence. The latter modules determine the positions of coding regions, the positions and scores of possible splice junctions, the positions of possible translationinitiation sites, the coding strand for the gene, and the probable-translation-frame function over the sequence. GAP tests combinations of those splice junctions which are within acceptable distances from the initial estimated edges of the coding regions. Every complete gene model, comprising translation-initiation site, splice junctions and stop codon, which agrees with GAP's set of rules is scored, and the ten highest-scoring models are saved. Each gene-model score depends on the input scores of splice junctions used in the model their positions relative to the initial estimated edges of the included coding regions, and the degree of agreement of the entire model with the probable-translation-frame function. If error conditions are detected, the present version of GAP attempts to correct them by the insertion and/or deletion of one or more coding regions. These insertions and deletions have resulted in a net improvement of gene models, and a particularly large improvement in the recognition and characterization of very short coding regions.

The results of GRAIL including the GAP module for 26 sequences from GenBank, each with a biochemically characterized single gene, are quite promising and demonstrate the feasibility of construction largely accurate gene models strictly on the basis of sequence data.

Research sponsored by U.S. DOE Office of Health and Environmental Research, and the Laboratory Directed Research and Development Programs.

${ }^{*}$ Biology Division.

3.56

\section{COMBINING NEURAL NETWORKS \\ AND EXPERT SYSTEMS TO IDENTIFY FEATURES IN DNA SEQUENCES}

\section{R. J. Mural ${ }^{*}$ X. Guan}
J. R. Einstein
E. C. Uberbacher

(Abstract of paper presented at the Eleanor Roosevelt Institute Transcribed Sequences Workshop, San Francisco, CA, October 31-November 1, 1992)

We have developed an on-line e-mail service, GRAIL, which locates the protein coding regions of DNA sequences. This interface utilizes a multiple sensor-neural network to find coding regions and a rule based interpreter to reduce this output to a table which includes other useful information such the identity of the coding strand and the preferred reading frame of the hypothetical coding exon. GRAIL can analyze up to $100 \mathrm{kbp}$ of sequence at a time and several sequences may be included in a single e-mail message. The analysis is performed on both strands of the input sequence and the interpreter assigns the putative coding region to a preferred strand and reading frame. In our experience the strand and reading-frame assignments are accurate about $95 \%$ of the time. GRAIL continues to find $90 \%$ of coding exons over 100 bases in length with a very low false positive rate. Currently GRAIL has about 500 users world-wide. Additional modules, which determine the position and quality of potential splice junctions, and the positions of potential translationinitiation sites, are being added to GRAIL. A 
Gene Assembly Program (GAP) is being developed which combines the outputs from the various GRAIL modules and attempts to predict the sequence of the spliced mRNA from the genomic DNA sequence. If error conditions are detected by the GAP rule-base, then the program attempts to correct them by the insertion and/or deletion of one or more coding exons. These actions result in a net improvement in gene-model prediction, particularly in the recognition and characterization of very short coding regions.

Research sponsored by U.S. DOE Office of Health and Environmental Research.

*Biology Division.

\title{
3.57
}

\section{SEARCHING FOR CONSENSUS PATTERNS ON A HYPERCUBE}

\author{
X. Guan \\ R. C. Mann \\ R. Mural* \\ E. C. Uberbacher
}

[Abstract of paper presented at the 6th Distributed Memory Computing Conference, Portland, OR, April 28-May 2, 1991; Proc. pp. 470-472 (1991)]

In DNA sequence analysis, consensus patterns (those that are not precisely conserved in location or with the same sequence of letters) are frequently sought among a number of sequences to find important biological features. Sequential algorithms for finding consensus patterns are timeconsuming due to the nature of the inexact occurrences of the patterns. Here we show that consensus pattern search can be done efficiently on the hypercube. We describe our implementation of two algorithms to find consensus patterns on an Intel iPSC/860 Hypercube and various techniques to speed up the computation.

Research sponsored by U.S. DOE Office of Health and Environmental Research.

*Bology Division. 
Section 4

NUCLEAR ANALYSIS AND SHIELDING 


\subsection{INTRODUCTION}

\section{T. Ingersoll}

The primary mission of the Nuclear Analysis and Shielding Section is to develop and apply state-of-the-art methods for the modeling of radiation transport and physics phenomena. The section is comprised of four groups whose major research activities include: (1) computational analysis and experiments for fission reactor shielding, (2) methods development and analysis for fission reactor core physics, (3) development of computer methods and models for high-energy radiation and particle transport and detection, and (4) mathematical modeling and computer analysis of ground- and spaced-based defensive systems. The Oak Ridge Detector Center, which is also organizationally contained in the section, facilitates ORNL's involvement in the development, design, and engineering of physics detectors for the Superconducting Super Collider (SSC).

A major accomplishment was achieved by the Reactor Shielding Group with the completion of the Japanese-American Shielding Program of Experimental Research (JASPER). This extensive program of measurements for the DOE Advanced LiquidMetal-Cooled Reactor Program was initiated in 1985, was suspended from 1987 through 1989 due to the administrative shutdown of the Tower Shielding Facility, and was finally completed in September of 1992 . Other activities within the group include (1) large-scale shielding analysis for the high-temperature gas-cooled reactor program and the liquid-metal-cooled reactor program, (2) initial analysis of neutron and gamma-ray shielding issues for the proposed Advanced Neutron Source (ANS), and neutronics analyses in support of the operation and the Safety Analysis Report for the ORNL High Flux Isotope Reactor.

The lead activity in the Reactor Physics Group focused on performance and design analyses for the ANS, including control concepts, fuel grading options, and nuclear heating. Numerous design parameterization and trade-off studies were performed to support the completion of the ANS Conceptual Design Report. A substantial program of methods development, benchmark analyses, and design methodology validation was established for the modular gas-cooled reactor (MHTGR) concept for the New Production Reactor (NPR) program, until the abrupt collapse of the that program. A down-scaled effort has continued for the commercial MHTGR program, including the on-site assignment of staff at the PROTEUS experimental facility in Switzerland. Work continues on the development and application of automated adjoint methods for sensitivity and uncertainty analysis, which is being successfully applied to low-level waste site performance assessment studies. Exploratory studies also continue for the adaptation of transport and diffusion methods to parallel computer architectures.

In the area of high-energy physics, there was a substantial growth in activities managed within the Oak Ridge Detector Center. The center, which operates in conjunction with other laboratories and universities in the southeast region of the U.S., coordinates basic research support of general high-energy detection systems and specific development and engineering support for the Solenoidal Detector Collaboration (SDC) and the Gamma-Electron-Muon (GEM) collaboration. Specific research within EPMD includes the analysis of several calorimeter designs and the application of neural networks to particle track analysis. Related work within the Applied Physics Group includes the prediction of radiation doses due to partial or 
full loss of the primary SSC beam and further improvements to the physics models and nuclear data bases for the HETC high-energy radiation and particle transport code.

The Advanced Systems Group continued to explor opportunities to develop new methods and to apply traditional methods to new upplications. Our long-term development and analysis support for the Defense Nuclear Agency continues, with the focus on: (1) improving the MASH code system, (2) validating the methodology against benchmark experiments, and (3) applying MASH for the prediction of radiation hardness for specific armored vehicles. As part of this effort, new graphical interfaces are being developed to aid the construction and display of threedimensional geometry models for transport analyses. The TORT three-dimensional discrete ordinates code continues to be developed and applied under an increasing diversity of sponsorships. The first TORT workshop was held, which was attended by over 90 individuals representing nearly 50 different organizations. Exploratory studies continued for the mathematical modeling of nonlinear systems, including the use of coupled nonlinear partial differential equations to describe the behavior of competitive systems.

NOTE: The Department of Energy has categorized much of our work for the Office of Nuclear Energy (NE) as "Applied Technology." Hence, explicit references to this work and the corresponding publications have not been included in this report. 


\section{FISSION REACTORS}

4.1

\section{DESIGN CALCULATIONS FOR THE ANS COLD NEUTRON SOURCE II: HEATING RATES}

\author{
R. G. Alsmiller, Jr. R. A. Lillie
}

[Abstract of Nucl. Instrum. Methods Phys. Res. (in press)]

Calculated results to aid in the design of a liquid deuterium cold source in the $\mathrm{D}_{2} \mathrm{O}$ reflector of a high-flux reactor are presented. The results presented include a comparison with experimental data, neutron leakage current as a function of wavelength, and heating rates in liquid deuterium and the other materials of the cold source.

Research sponsored by U.S. DOE Office of High Energy and Nuclear Physics.

\section{2}

\section{SIMULATION OF THE PULSED NEUTRON SOURCE EXPERIMENT}

\section{Caro* F. C. Difilippo}

(Abstract of ORNL/M-2157, June 1992)

This report describes a Fortran code named SIMIL which simulates the Pulsed Neutron Source Experiment. This computational tool has been specially developed to help validate the analysis software and to investigate its sensitivity to parametric uncertainties, It will also help to determine the best experimental conditions under which the experiment should be done in each subcritical case.

Research sponsored by U.S. Department of Energy.

*Paul Scherrer Institute, Villigen Switzerland.
4.3

\section{APPLICATION OF 3-DIMENSIONAL RADIATION TRANSPORT CODES TO THE ANALYSIS OF THE CRBR PROTOTYPIC COOLANT PIPE CHASEWAY NEUTRON STREAMING EXPERIMENT}

\author{
K. Chatani*
}

(Abstract of ORNL/TM-11641, August 1992)

This report summarizes the calculational results from analyses of a Clinch River Breeder Reactor (CRBR) prototypic coolant pipe chaseway neutron streaming experiment. Comparisons of calculated and measured results are presented, major emphasis being placed on results at bends in the chaseway.

Calculations were performed with three threedimensional radiation transport codes: the discrete ordinates code TORT and the Monte Carlo code MORSE, both developed by the Oak Ridge National Laboratory (ORNL), and the discrete ordinates code ENSEMBLE, developed by Japan. The calculated results from the three codes are compared (1) with previously-calculated DOT3.5 two-dimensional results, (2) among themselves, and (3) with measured results. Calculations with TORT used both the weighted-difference and nodal methods. Only the weighted-difference method was used in ENSEMBLE.

When the calculated results were compared to measured results, it was found that calculationto-experiment $(C / E)$ ratios were good in the regions of the chaseway where two-dimensional modeling might be difficult and where there were no significant discrete ordinates ray effects. Excellent agreement was observed for responses dominated by thermal neutron contributions. MORSEcalculated results and comparisons are described also, and detailed results are presented in an appendix.

Research spensored by U.S. Department of Energy.

"Power Reactor and Nuclear Development Corporation, Ibaraki, Japan. 
4.4

\section{CROSS SECTIONS FOR ACTINIDE BURNER. REACTORS}

\author{
F. C. Difilippo
}

[Abstract of paper presented at the International Conference on Nuclear Data for Science and Technology, Julich, Federal Republic of Germany; May 13-17, 1991, Proc. pp. 95-97, S. M. Qaim. Ed., Springer-Verlag (1992)\}

Recent studies have shown the feasibility of burning higher actinides [i.e., transuranium (TRU) elements excluding plutoniun! in ad hoc designed reactors (Actinide Burner Reactors: ARR) which, because of their hard neutron spectra, enhance the fission of TRU. The transmutation of long-lived radionuclides into stable or short-lived isotopes reduces considerably the burden of handling higli-level waste from either LWR or Fast Breeder Reactors (FBR) fuels. Because of the large concentrations of higher actinides in these novel reactor designs the Doppler effect due to TRU materials is the most important temperature coefficient from the point of view of reactor safety. Here we report calculations of energy group-averaged capture and fission cross sections as function of temperature and dilution for higher actinides in the resolved and unresolved resonance regions. The calculations were done with the codes SAMMY in the resolved region and URR in the unresolved regions and compared with an independent calculation.

Rescards sponsored by l:s. Department of Energy:

4.5

\section{EFFECTS OF THE TWO-FISSION. NEUTRON DISTRIBUTIONS IN REACTIVITY MEASUREMENTS WITH THE ${ }^{252}$ CF SOURCE}

\section{F. C. Difilippo}

Abstract of paper presented at the International Conference on Nuclear Data for Science and Technology. Julich, Federal Republic of Ciermany, May 13-17, 1991; Proc. pp. 122-123, S. M. Qaim, Ed., Springer-Verlag (1992)]

Stochastic descriptors based on the first and second moments of the probability distribution of the neutrons in phase space $\vec{\mu}=(\vec{r}, \vec{v})$ are extensively used in the nuclear industry for the nonintrusive measurement of nuclear paraineters. One of these methods is based on the ${ }^{252} \mathrm{Cf}$ source which has been used in an unprecedented range of situations and reactivities, the interpretation of this method is sensitive to the distribution of two neutrons coming from the same fission, $i\left(\bar{v}, \bar{v}^{\prime}\right)$. The few direct measurements of this $n-n$ correlation have shown that $\gamma$ depends on $\cos \theta^{\prime \prime}=$ $\bar{v} \cdot \bar{v}^{\prime} \mid v v^{\prime}$ and the neutron energies, the availaile experimental data was used to quantify the sensitivity of the ${ }^{252} \mathrm{Cf}$ method to the anisotropies of the $n-n$ correlation. It was found that it affects the interpretation of the reactivity measurement for low values of the multiplication constant.

Research spousored 1; (i.S. Department of Energy:

\section{6}

\section{LEU-HTR PROTEUS: THEORY AND SIMULATIONS OF REACTIVITY MEASUREMENTS WITH THE INVERSE KINETICS METHOD}

\section{F. C. Difilippo}

(Abstract of ORNL/M-2156, June 1992)

The experimental program for low enriched high-temperature gas-cooled reactor configurations in PROTEUS includes the measurement of the reactivity worth of control rods using the inverse kinetics method. We summarize the theoretical basis of the method to show how spatial effects can be calculated to design the experiment and to interpret the measurements; the results are illustrated with example of LEU-HTR PROTEUS configurations calculated with computer codes in use at PSI and with the analysis of computer generated "experimental" data.

Research sponsored by U.S. Department of Energy. 
4.7

\section{LEU-HTR PROTEUS: NEUTRON \\ KINETICS AND PROCEDURES TO ANALYZE PULSED NEUTRON EXPERIMENTS}

\author{
F. C. Difilippo
}

(Abstract of ORNL/M-2154, June 1992)

The definition and measurement of kinetics parameters in systems heavily reflected with very low absorbing materials exhibit additional complications because of the different distributions of prompt and delayed neutrons. After consistent definitions of these parameters the pulsed neutron techniques to measure reactivities are discussed to find correction factors for spatial effects or to locate the detectors to minimize them. The theoretical results were implemented using codes available at PSI in order to analyze future measurements at the PROTEUS facility to simulate low enriched high temperature gas cooled rcactor configurations.

Research sponsored by U.S. Department of Energy.

\section{8}

\section{PNS MEASUREMENTS OF EFFECTIVE GRAPHITE ABSORPTION}

\section{F. C. Difilippo}

(Abstract of ORNL/M-2155, June 1992)

A pulsed neutron source (PNS) is currently being used to assess the amount of equivalent ${ }^{10} \mathrm{~B}$ impurities in the graphite of the PROTEUS facility. A crucial step in the analysis of the experiment is the comparison of measured values of the decay constant $(\alpha)$ with calculations in order to determine the amount of ${ }^{10} \mathrm{~B}$. We report calculated $\alpha$ values for complicated graphite configurations as well as their sensitivities to the amount of ${ }^{10} \mathrm{~B}$, structural materials and the presence of the source.

Research sponsored by U.S. Department of Energy.

4.9

\section{TEMPERATURE-DEPENDENT BONDARENKO FACTORS FOR ${ }^{237} \mathrm{~Np},{ }^{241} \mathrm{Am}, \mathrm{AND}{ }^{243} \mathrm{Am}$}

F. C. Difilippo

(Abstract of ORNL/TM-11727, April 1991)

Energy group averaged capture and fission cross sections were computed for ${ }^{237} \mathrm{~Np},{ }^{241} \mathrm{Am}$, and ${ }^{243} \mathrm{Am}$ as function of temperature and dilution in the resolved and unresolved resonance regions. The computer codes SAMMY and URR (actively used for the analysis of cross sections measurements) were used for this purpose.

Research sponsored t: U.S. Department of Energy.

4.10

\section{TEMPERATURE-DEPENDENT BONDARENKO FACTORS FOR ${ }^{243} \mathrm{Cm},{ }^{244} \mathrm{Cm}$, AND ${ }^{245} \mathrm{Cm}$}

\author{
F. C. Difilippo
}

(Abstract of ORNL/TM-11837, May 1991)

The computer codes SAMMY and URR were used to calculate energy group averaged capture and fission cross sections of ${ }^{243} \mathrm{Cm},{ }^{244} \mathrm{Cm}$, and ${ }^{245} \mathrm{Cm}$ in, respectively, the resolved and unresolved resonance regions. Bondarenko factors were then tabulated as a function of temperature and dilution.

Research sponsored by U.S. Department of Energy. 
4.11

\section{STOCHASTIC PROCESSES IN NUCLEAR REACTORS UNDER PARAMETRIC EXCITATION}

\author{
F. C. Difilippo K. Macko*
}

[Abstract of paper presented at the American Nuclear Society Topical Meeting on Advances in Mathematics, Compu. tation and Reactor Physics. Pittsburgh, PA, April 28-May 1. 1991; Proc. Vol. 1. p. 5.2. 1.1 (1991)]

The statistical properties of a neutron field subject to parametric excitations are analyzed. The goal is to determine under what conditions noise techniques, developed for steady state systems, can be applied to measure properties of systems that are changing in time.

Research sponsored by U.S. Department of Energy:

*Allegheny College, Nieadville, PA.

\subsection{2}

\section{APPLICATION OF A NEW INTEGRAL REPRESENTATION OF THE $\mathrm{K}_{0}$ BESSEL FUNCTION}

\section{F. C. Difilippo R. B. Perez*}

[Abstract of Appl. Math. Lett. 5(5), 51 (1992)]

The analysis of either the noise signatures of boiling water reactors or the homogeneous equivalence of a nuclear reactor fuel cell require the analysis of the propagation of neutron waves along the fuel rods embedded in a moderator. During the course of our work, we have found a convenient integral representation of the $K_{0}$ Bessel function that allowed us to find the relaxation length of the neutron waves.

Research sponsored by the U.S. Department of Energy.

\footnotetext{
*Instrumentations and Controls Division.
}

4.13

\section{STATUS AND COVARIANCE FILES OF THE SCATTERING MATRIX OF GRAPHITE}

\author{
F. C. Difilippo J. P. Renier* \\ B. A. Worley
}

(Abstract of paper presented at the Symposium on Nuclear Data Evaluation Methodulogy, Brookhaven National Laboratory, Upton, NY. Octuber 12-16, 1992)

Calculations related to the temperature coefficient of reactivity of enriched gas-cooled reactors show the high sensitivity of this parameter to the proper description of thermalization effects in the moderator: additionally, calculation of the temperature dependence of the inelastic scattering cross section correlates the errors of the cross sections as functions of the temperature. The ENDF/B evaluation of $S(\alpha, \beta, T)$ is still based on the approximations (incoherent component only) and phonon spectras of the early 60's. Subsequent measurements showed that the structure observed in $S(\alpha, \beta, T)$ cannot be described using the incoherent approximation. and soon after the availability of highly intense neutron beams and large specimens of pyrolitic graphite better calculation of the phonon spectra were possible. Our calculations of the probability distributions of the mo mentum and energy transfer, $\alpha$ and $\beta$, allow one to define a range of $\alpha$ and $\beta$ for which comparison of experimental and theoretical data is of most interest for reactor analysis, and to point out regions of deficient resolution or excessive details in the present $\alpha, \beta$ mesh used in the ENDF/B files. Because the phonon spectra defines $S(\alpha, \beta, T)$ we have found mathematical formulas that allow the calculation of the covariance matrix of $S$ by propagating the errors of the phonon spectra.

Research sponsoled by U.S. Departnient of Energy.

*Computing and Teleconumunications Division. 


\subsection{4}

\section{FRAMEWORK FOR MONITORING RELIABILITY}

\author{
M. J. Haire* M. S. Smith \\ D. R. Moser ${ }^{\dagger}$
}

(Abstract of paper presented at the 1992 ANS/ASME Nu
clear Energy Conference, San Diego, CA, August 23-26, clear Energy Conference, San Die
1992; Proc. pp. 179-182 (1992)]

A personal computer (PC) based data collection system under development for the Toxic Substances Control Act (TSCA) Incinerator at the K. 25 site in Oak Ridge is proposed as a framework for monitoring the effectiveness of maintenance activities at commercial nuclear power plants. The U.S. Nuclear Regulatory Commission (NRC) has recently required the analysis and documentation of every significant plant equipment malfunction. relative to plant safety. The volume of data will require a structured data recording and retrievai system, much like that under development for the TSCA incinerator.

Research sponsored by U.S. Department of Energy.

*Robotics and Procmss System Divisian.

${ }^{\dagger}$ Toxic Subssiances : Ontrol Act lncinerator, Oak Ridge Gaseous Diffusior. P! at.

\subsection{5}

CALCULATED ENERGY AND ANGULAR DEPENDENCE OF PARTICLE FLUXES AT THE EXIT OF THE ADVANCED NEUTRON SOURCE RADIAL AND TANGENTIAL BEAM TUBES

\section{Hayashi* ${ }^{*}$ T. Nishigori ${ }^{\dagger}$}

R. G. Alsmiller, Jr. R. A. Lillie

[Abstract of Nucl. Sci. Eng. (in press)]

Calculational studies have been carried out of neutron and gamma-ray transport in the beam tubes of a proposed high flux reactor for the Advanced Neutron Source. To avcid excessively long computing times, the calculations were carried out by coupling two two-dimensional discrete ordinates calculations. The calculational methodology used is briefly described.

Calculated results are presented of the thermal neutron, non-thermal neutron, and gamma- ray fluxes in a radial and a tangential beam tube. Both scalar and angular fluxes in and at the exit of the beam tubes are given. When compared with the radial beam tube, the tangential beam tube shows a lower flux of high-energy neutrons and a much lower flux of gamma rays. The spectral characteristics of the particle fluxes at the exits of the beam tubes are similar to those at the entrances.

Research sponsored by U.S. DOE Office of Basic Energy Sciences.

*Kyoto Universit., Osaka, Japan.

†Osaka University, Osaka, Japar.

\subsection{6}

\section{EARLY TEST FACILITIES AND ANALYTIC METHODS FOR RADIATION SHIELDING}
D. T. Ingersoll
J. K. Ingersoll

(Abstract of ORNL/RSIC-55. November 1992)

This report represents a compilation of eight papers presented at the 1992 American Nuclear Society/European Nuclear Society International Meeting held in Chicago, Illinois on November 1520,1992 . The meeting is of special significance since it commemorates the 50th anniversary of the first controlled nuclear chain reaction. The papers contained in this report were presented in a special session organized by the Radiation Protection and Shielding Division in keeping with the historical theme of the meeting. The papers describe several early shield test facilities, experimental programs, and method development efforts as presented from the personal perspective of the early pioneers.

Research spunsored by the U.S. Department of Energy.

*Tec-Com, Inc., Knoxville, TN. 
4.17

\section{PHASE II TESTING OF ENDF/B-VI SHIELDING DATA}

\author{
D. T. Ingersoll R. Q. Wright* \\ C. O. Slater
}

[Abstract of paper presented at the American Nuclear Society Topical Meeting on New Horizons in Radiation Protection and Shielding, Pasco, WA, April 26-30 (1992); Proc. pp. 385-391 (1992)]

Version 6 of the U.S. Evaluated Nuclear Data File (ENDF/B-VI) was released in early 1990 and is currently undergoing phase II testing. In Phase II testing, the evaluated data are approximately processed and used in an integral manner to predict the solution of previously specified bencinmark experiments. Results are presented for the initial testing of several light elements and structural materials which are important for shielding applications. These initial tests indicate that the relatively subtle changes made to the iron data and the major modernization of the boron-11 data in Version 6 both represent significant and positive advancement in the quality of the evaluated data files.

Research sponsored by U.S. Department of Energy.

*Computing and Telerommunications Division.

4.18

\section{NEEDS FOR EVALUATED COVARIANCE DATA FOR REACTOR PRESSURE VESSEL DOSIMETRY}

\section{R. E. Maerker $\quad$ B. L. Broadhead* J. J. Wagschal ${ }^{\dagger}$}

Abstract of paper presented at the Nuclear Energy Agency Nuclear Science Committee Specialists' Meeting on Evaluation and Processing of Covariance Data, Oak Ridge National Laboratory, Oak Ridge, TN, October 7-9, 1992; Proc. pp. 13-32 (1993)]

A new methodology for quantifying and then reducing uncertainties in the calculated pressure vessel fluences of a pressurized water reactor (PWR) is reviewed. The technique involves combining the integral results of the calculated and measured PWR surveillance dosimetry activities with the differential data used in the calculations, along with covariances of all the quantities, into a generalized linear least-squares adjustment procedure. Based on analysis of both PWRs and test reactor benchmarks, substantial evidence now exists to support the conclusion that, of all the nuclear as well as non-nuclear differential data considered, ENDF/B-V values of the total inelastic iron cross sections and their covariances are the most important data controlling the outcome of the adjustment procedure. Predicted adjustments in these cross sections provided the stimulus for new measurements, the results of which impacted the ENDF/B-VI evaluation of iron 56.

Research sponsored by U.S. Department of Energy.

*Computing and Telecommunications Division.

${ }^{\dagger}$ Hebrew University, Jerusalem, Israel.

4.19

\section{REACTOR PHYSICS INPUT TO THE SAFETY ANALYSIS REPO:T FOR THE HIGH FLUX ISOTOPE REACTOR}

\author{
R. T. Primm, III
}

(Abstract of ORNL/TM-11956, March 1992)

HFIR specific, few group neutron and coupled neutron-gamma libraries have been prepared. These are based on data from ENDF/B-V and beginning-of-life (BOL) conditions. The neutron library includes actinide data for curium target rods. Six critical experiments, collectively designated HFIR critical experiment 4 , were analyzed. Calculated $k$-effective was $2 \%$ high at BOL-typical conditions but was 1.0 at end-of-life-typical conditions.

The local power density distributions were calculated for each of the critical experiments. The axially averaged values at a given radius were frequently within experimental error. However at individual points, the calculated local power densities were significantly different from the experimentally derived values (several times greater than experimental uncertainty). A reassessment of the foil activation data with transport theory techniques seems desirable.

Using the results of the critical experiments study, a model of the current HFIR configuration was prepared. As with the critical experi- 
ments, BOL k-effective was high (3\%). However, end-of-life k-effective was also high (2\%). The end-of-life concentraticrs of fission products were compared to those generated using the ORIGEN code. Agreement was generally good through differences in the inveutories of some important nuclides. Xe and I, need to be understood. End-ofcycle curium targec isotopics based on measured, discharged target rods were compared to calculated values and agreement was good.

Axial flux plots at varicus irradiation positions were generated. Time-dependent power distributions based on two-dimensional calculations were provided.

Research sponsured by U.S. Deparment of Energy:

\subsection{0}

\section{CALCULATION OF CRITICAL EXPERIMENT PARAMETERS FOR THE HIGH FLUX ISOTOPE REACTOR}

\author{
R. T. Primm, III
}

[Abstract of paper presentel at the American Nuclear So ciety Topical Meeting on Advances in Reactor Physics, Charieston. SC, March 8-11. 1992; Proc. Vol. 2, pp. 203$218(1992)]$

Six critical experiments were performed shortly before the initial ascension to power of the High Flux Isotope Reactor (HFIR). Critical configurations were determined at various control rod positions by varying the suluble boron content in the light water $c$ olant. Calculated $k$-effective was $2 \%$ high at beginning-of-life (BOL) typical conditions, but was $1 . J$ at end-of-life (EOL) typical conditions. Axially averaged power distributions for a given radial location were frequently within experimental error. At specific $r, z$ locations within the core, the calculated power densities were significantly different from the experimentally derived values. A reassessment of the foil activation data seems desirable.

Research sponsored by L.S. Departrient of Energy.

\subsection{1}

$$
\begin{aligned}
& \text { CRITICALITY SAFETY STUDIES } \\
& \text { FOR THE STORAGE OF WASTE } \\
& \text { FROM NUCLEAR FUEL SERVICES } \\
& \text { IN INTERCELL STORAGE WELLS } \\
& 2 \text { AND } 3 \text { OF BUILDING } 3019
\end{aligned}
$$

\author{
R. T. Primm, III \\ G. R. Smolen*
}

(Abstract of ORNL/TM-12152, November 1992)

This report provides computational evaluation results demonstrating that mixed oxide waste can be safely stored in Intercell Storage Wells 2 and 3 of Building 3019 at the Oak Ridge National Laboratiory. Existing, verified computational techniques are validated with applicable critical experiments and tolerance limits for safety analyses are derived. Multiplication factors for normal and credible abnormal configurations are calculated and found to be far subcritical when compared ' 0 derived safety limits.

Research sponsored by U.S. Department of Energy.

* Computing and Telecammunicaticns Division.

4.22

\section{AN ASSESSMENT OF LIQUID METAL CENTRIFUGAL PUMPS AT THREE FAST REACTORS}

\author{
M. S. Smith D. H. Wood \\ J. D. Drischler
}

(Abstract of Nuclear Technology American Nuclear Society (in press)]

This paper describes the results of an analysis using data reports submitted to the Centralized Reliability Data Organization (CREDO) to predict the onset of the wearout life period for large sodium centrifugal pumps. For CREDO data collection and analysis purposes, a "rnechanical pump" includes the purnping unit, its driver, and the coupling between the two. Statistical data were compiled from event reports received from three fast reactors: the Experimental Breeder Reactor II (EBR-II) and the Fast. Flux Test Facility (FFTF) in the United States, and the JOYO Experimental Fast Reactor operated by the Power Reactor and Nuclear Fuel Drvelopment ('orpora- 
tion (PIC) of Japan. Results from EBR-[I indicate there is a definile time dependent relationship between event rates and punip age; the common event mode al EBR-II is pump binding or seizing due to the buildup of sodium deposits in the vicinity of the lower labyrinth seal. There is no indication from FFTF that the six centrifugal pumps have reached the end of their useful life: these pumps have becn event free for nearly 40.000 operating hours. After 50.000 hours of event free operation at $\mathrm{JOYO}^{\circ} \mathrm{O}$, bearings in the secondary pumps required additional unsclieduled maintenance. It is premature to conclude that these pumps have entered into the wearout life period: more data is required to draw any such conclusion. Cumulative event rates were calculated for the investigated pumps at each facility and for the entire population. For all pumps, thc evellt rate was computed as 34.4 events per million operating hours with $5 \%$ and $9.5 \%$ one-sided confidence limits of 26.3 and 44.4 events per million operating hours. respectively: The cummlative event rates for EBR-II, FFTF, and JOYO. Were computed as 30.0. 32.4. and 40.6 events per million plimp operating hours. respectively:

Research sponsored by (․:. Department of Energy:

\subsection{3}

\section{SYSTEM MODEL DEVELOPMENT FOR NUCLEAR THERMAL PROPULSION}
J. T. Walton*
K. R. Perkins
N. A. Hannan ${ }^{\dagger}$
B. A. Worley
J. J. Buksa ${ }^{\natural}$
D. Dobranich \#

(Abstract of paper presented at the 43rd Congress of the International Astronautical Federation. Washington, DC, August 28-September 5, 1992;

A critical enabling technology in the evolutionary development of nuclear thermal propulsion (NTP) is the ability to predict the system performance under a variety of operating conditions. This is crucial for mission analysis and for control subsystem testing as well as for the modeling of various failure modes. Performance must be accurately predicted during steady-state and transient operation. including startup, shutdown and post operation conling. The development and application of verified and vaiidated systein models lias the porential to realuce the dcsign, lesting. cost and time required for the technology to reach flight-ready status.

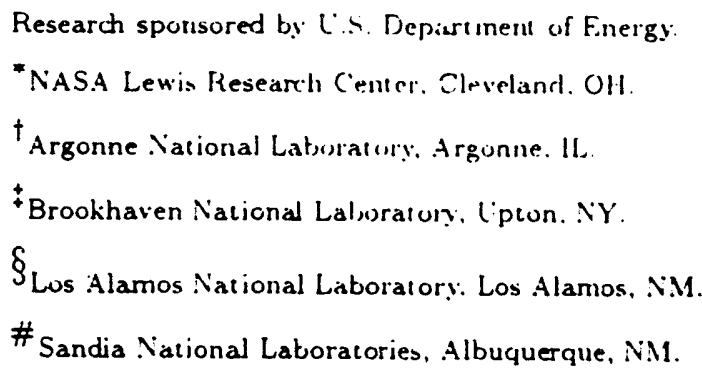

4.24

\section{MOTOR-OPERATED GLOBE VALVE PERFORMANCE IN A LIQUID SODIUM ENVIRONMENT}

\author{
D. H. Wood M. S. Snith \\ J. D. Drischler
}

[Abstract of paper presented at the 1992 ANS/ASME Nuclear Energ. Conference. San Diego. CA. August 23-26. 1992; Proc. pp. 341-346 (1992)]

This study investigates motor-operated globe valve (MOV) performance in a liquid sodium environment as reported to the ('entralized Reliability Data Organization (CREDO) from site representatives at several liquid metal reactors and liquid metal test facilities. The CREDO dat a base contains engineering histories for 179 motor-operated glnt, valves. Thirty nine failures have been documented for these components in over $8.7 \mathrm{mil}$ lion hours of operation. The most common MOV events were anomalies with the limit and torque switches, although humau initiated problems were also frequent causes of failures. The failure data suggest that an improved preventive maintenance program with a higher frequency of inspection of the limit and torque switches should increase MOV availability and reliability. The event rate for all failure modes was compuled as $4.4 T$ events per $10^{6}$ operating hours by assuming a Poisson distribution of failures over valve operating time. The $5 \%$ and $9.5 \%$ confidence limits based on a chi-squared $\left(\chi^{2}\right)$ probability distribution function were computed as 3.36 and 5.83 events per $10^{6}$ operating hours, respectively. The operating performance of these liquid thetal MOV's was compared to similar data for MON's in commercial light v:ater reactors and was foumd to exhibit similar failure ratis.

Keseard sponsured by $L:$. Departunent of Energy: 


\section{HIGH-ENERGY ACCELERATORS AND DETECTORS}

4.25

\section{LIQUID SCINTILLATOR CALORIMETRY (SECOND YEAR) A RESEARCH AND DEVELOPMENT RENEWAL PROPOSAL TO THE SUPERCONDUCTING SUPER COLLIDER LABORATORY}
E. Ables ${ }^{*}$...
B. L. Bishop ${ }^{\ddagger}$
T. A. Gabriel
Y. Kamyshkov $§$ F. Plasil $§$

M. L. Bauer ${ }^{\dagger}$

H. O. Cohn $\S$

A. Gordeev $\S$

R. A. Lillie

K. Read $\S$

M. J. Rennich\#

\section{A. Savin $\S$}

K. Shmakov $§$

A. Smirnov $\S$

B. H. Singeltary \#

R. A. Todd ${ }^{\dagger}$

E. Tarkovsky $\S$

K. G. Young ${ }^{\dagger}$ et al.

(Abstract of ORNL/M-1774, September 1991)

Precision electromagnetic and hadronic calorimeters are among the major tools for studying the new physics in experiments at the SSCL. A liquid scintillator calorimeter can provide this precision. Presented in this report is a descriptior. of our research on liquid scintillator calorimetry, which includes simulation, materials compatibility, radiation damage, light output, electrical and mechanical engineering studies, etc. So far all of our research indicates that this technology offers excellent potential for use at the SSCL.

Research sponsored by U.S. DOE and the Superconducting Super Collider Laboratory.

*Lawrence Livermore National Laboratory, Livermore, CA.

†nstrumentation and Contols Division.

${ }^{\ddagger}$ Computing and Telecommunications Division.

$\oint_{\text {Physics Division. }}$

\# MMES Engineering.
4.26

\section{HECC92, AN UPDATED \\ INTRANUCLEAR CASCADE MODEL OF HIGH-ENERGY \\ HADRON-NUCLEUS COLLISIONS}

F. S. Alsmiller R. G. Alsmiller, Jr.

O. W. Hermann*
[Abstract of paper presented at the Southeastern Section Meeting of the American Physical Society. Oak Ridge, TN, November 12-14, 1992; Proc. Vol. 37(7), p. 1673 (1992)]

The new time-dependent high-energy intranuclear cascade code, $\mathrm{HECC} 92$, (based on the original HECC of Bertini et al.), incorporates the hadron-nucleon dual parton fragmentation model of $\mathrm{J}$. Ranft and collaborators from the transport code FLUKA8i for high momentum $(>10 \mathrm{GeV} / \mathrm{c}$ in the target nucleon rest system) inelastic hadronnucleon collisions. At lower entergies, the isobar model HADRIN, from FLCKAS is used for iso bar production and decay. It permits single and double isobar production. with a maximum of 100 stable particles after the isohar decay. This replaces the isobar model of Sternheimer previously used at energies below $\sim 3$ Ciel'. Comparisons are made with experimental data in the energy range 2.7 to $50.0 \mathrm{GeV}$. The concept of optional formation times for particles produced in IIADRIN and in the fraginentation model lias been introduced for study at higher incident entrgies. This is now thought necessary to decrease the high multiciplicities of produced particles, so as to agree with experiment.

Researdi spousnred by U:S. DOE Office of High Energo and Nuclear Physics.

\footnotetext{
* Computing and Telecumumunications Divisinn.
} 
4.27

\section{THE USE OF DELAY TIMES IN THE INTRANUCLEAR CASCADE MODEL OF HIGH-ENERGY ( $\sim 5 \mathrm{GeV})$ HADRON-NUCLEUS COLLISIONS}

\author{
F. S. Alsmiller R. G. Alsmiller, Jr. \\ O. W. Hermann*
}

Abstract of paper presented at the American Nuclear Society Topical Meeting on New Horizons in Radiation Protection and Shielding, Pasco, WA, April 26-30 (1992); Prec. pp. 505-508 (1992)]

The concept of formation times for particles produced in hadron-hadron collisions inside the nucleus has been used in conjunction with the intranuclear cascade model to study particle production in hadron-nucleus collisions. The model used also allows for the depletion of nuclear matter.

Comparisons are made with experimental data in the energy range 12.5 to $50 \mathrm{GeV}$, and it is concluded that additional comparisons will be needed before definite model parameters are obtained.

Research sponsored by U.S. DOE Office of Hight Energy and Nuclear Physics.

* Computing and Telecornmunicatiuns Division.

\subsection{8}

\section{PARTICLE PRODUCTION MODELS IN HETC88 IN THE ENERGY RANGE 3 TO $30 \mathrm{GeV}$}
R. G. Alsmiller, Jr.
F. S. Alsmiller

IAbstract of paper presented at the American Nuclear Society Topical Meeting on New Horizons in Radiation Protection and Shielding, Pasco, WA, April 26-30 (1992); Proc. pp. $499-504$ (1992)]

In HETC88, a parameter ESKALE determines which of the two particle production models, i.e. a scaling or a multi-chain fragmentation model is used. This abrupt change of models at a specific energy can lead to discontinuous results. In this paper, alternative to this procedure based on using an energy dependent linear combination of the models is proposed. It is concluded that the version of HETC88 proposed here is preferable to HETC88 with ESKALE $=5 \mathrm{GeV}$ for calculating average quantities but may not be preferable for calculating the fluctuations about average quantities.

Research sponsored by U.S. DOE Office of High Energy and Nuclear Physics.

\subsection{9}

THE SUPER FIXED TARGET
BEAUTY FACILITY AT THE SSC
S. Anassontzis*
H. Brashear ${ }^{\dagger}$
N. Ericison ${ }^{\dagger}$
T. A. Gabriel

T. Moore ${ }^{\dagger}$ A. Wintenberg ${ }^{\dagger}$ et al.

(Abstract of paper presented at the Third Topical Seminar on Heavy Flavours, San Miniato, Italy, June 17-21, 1991)

The rationale for pursuing beauty physics at the SSC in a fixed target configuration is described. The increased beauty production cross section at the SSC, combined with high interaction rate capability of the proposed detector, results in 10 produced $B B$ events per year. The long decay length of the $B$ hadrons $(\simeq 10 \mathrm{~cm}$ ) allows direct observation of $B$ decays in the high resolution silicon microstrip vertex detector. To optimize the operation of the proposed beauty spectrometer and the SSC, parasitic extra:tion of attendant or artificially generated large amplitude protons using crystal channeling is proposed and explored. The large sample of fully reconstructed B events allows detailed studies of various $C P$ violating decays with requisite statistics to confront the standard model. The CP physics potential of the proposed experiment is evaluated and compared with alternative approaches, such as asymmetric $\mathrm{e}^{+} e^{-}$ $B$ Factories and specialized hadron colliders.

Research sponsored by U.S. Department of Energy.

*University of Athens, Athens, Greece.

$\dagger_{\text {Instrumentation and Controls Division. }}$ 
4.30

\section{LIQUID ARGON CALORIMETER DESIGN STUDY USING THE CALOR.89 CODE SYSTEM}

\author{
C. Y. Fu \\ R. G. Alsmiller, $\check{3} "$
}

T. A. Gabriel

[Abstract of Nucl. Instrum. Methods Phys. Res. A311, i79 (1992)]

The CALOR89 code system, already in wide use, is further validated against the recently available data for a test module of the calorimeter of the DO detector, made of uranium absorbers and liquid argon. The experimental data considered include energy contaimment as a function of depth, the electron and pion resolutions. and the compensation ratios at 25 and $100 \mathrm{Gel} / \therefore$. The code system is then used to predict the performance of a Pb-LAr test module under construction and to test the expected performance improveinent of a second PL-LAr module with steel-cladded $\mathrm{Fb}$ ab. sorbers. It is found that with a fixed total absorber thickness of $12 \mathrm{~mm}$ and LAr thickness of $4 \mathrm{~mm}$, varying the thickness of the steel cladding has minor effects on the compensation ratios. However, for $20 \mathrm{GeV} / \mathrm{c}$ incident electrons and $0.5-\mathrm{mm}$ steel cladding, there is a significant intprovement in en. ergy resolution

Rusearch sponsor.xi !y U.S. DOE High Encray Fhysics

4.31

\section{THE COMPUTATIONAL CHALLENGES PROVIDED BY THE CODES USED TO DESIGN AND ANALYZE SUPERCONDUCTING SUPER COLLIDER DETECTORS}

\section{T. A. Gabriel}

Abstract of paper presented at the Workshop on Undergraduate and Graduate Education, Louisiana State University. Baton Rouge, LA, April 20-30, 1991; Proc. pp. 105-117 (1991)]

"The Superconducting Super Collider (SSC) project is a critical element of our government's initiative to strengthen the position of the U.S. as a world leader in education, science and technology." This project is currently offering many challenges for the computational scientist and will, during the next decades, offer many more. During this presentation, I would like to discuss one of these challenges which deals with the codes used to design and analyze the large detectors that are needed to make the SSC a major success. In addition, I will address an area in which national laboratories like ORNL can assist in the education of computational scientists. To be specific, the following topics will be covered during this talk.

First of all, I would like to show you a generic model of an SSC detector and briefly describe the major components and their functions. This will give you a feel for the magnitude of the project that is charged with the design of SSC detectors and for the need of extremely good high energy physics detector simulation codes.

Secondly, I would like to overview the CALOR89 code system which is one of the recommended detector simulation codes for use at the SSC. This is an analog of the Monte Carlo Code System for those of you who have experience in this area.

Thirdly, I would like to discuss the computatonal problems associated with CALOR89 and some potential solutions. It is not very difficult to name a few problems-not very user friendly and requires large amounts of $\mathrm{CPU}$ time; and their potential solution - create user interfaces/menu driven input opticns and add more CPUs with greater speed.

Finally, I would like to address a potential role that national iaboratories can play in the education of the computational scientist.

Research sponsored by U.S. DOE and by the Office of Naval Research. 


\subsection{2}

\section{COMPARISON OF EXPERIMENTAL AND CALCULATIONAL ENERGY DEPOSITION PROFILES IN CALORIMETERS AND OTHER DAMAGE RELATED DATA}

\author{
T. A. Gabriel T. Handler* \\ P. K. Job ${ }^{\dagger}$
}

(Abstract of paper presented at the International Conference on Radiation-Tolerant, Florida State University, Tallahassee, FL, April 2s-May 2, 1992)

A comparison between the experimental data known as the "hanging file" and calculated results obtained using CALORS') on longitudinal "energy deposition" profiles (is modified for light emission) for various calorimeter configurations is presented.

Research sponsored by U.S. Department of Energy.

*University of Tennessec, Knuxville, TN.

†Argonne Nationis! Luboratory, Chicago, IL.

\subsection{3}

\section{CHARGED PARTICLE TRACK RECONSTRUCTION USING ARTIFICIAL NEURAL NETWORKS}

\author{
C. W. Glover \\ C. Y. Fu \\ T. A. Gabriel \\ T. Handler*
}

(Abstract of paper presented at the Conference on Computing in High-Energy Physics, Annecy, France, September $21-25,1992$ )

This paper summarizes the current state of our research in developing and applying artifcial neural network (ANN) algorithms to construct charged particle tracks from tracking chamber data. The ANN algorithm described here is based on a crude model of the retina. It takes as input the coordinates of each charged particle's interaction point ("hit") in the tracking chamber The algorithm's output is a set if vectors pointing to other hits that are most likely to form a track. Researdi sponsured ty U.S. DOE and the Superconducting
Super Collider Laboratory.

* University of Tenuessce. Inuxville. TN.
4.34

\section{HYBRID CENTRAL TRACKING CHAMBER COLLABORATION - PROGRESS REPORT FOR FY 1991}
A. T. Goshaw*...
G. T. Alley ${ }^{\dagger}$
T. A. Gabriel
C. W. Glover
C. L. Britton, Jr. ${ }^{\dagger}$
H. R. Brashear ${ }^{\dagger}$ T. Ryan ${ }^{\ddagger}$ et al.
D. Vandergriff ${ }^{\ddagger}$
M. Emery ${ }^{\dagger}$

(Abstract of ORNL/M-1775, September 1991)

This Progress Report for FY1991 is submitted to the SSC Laboratory in partial fulfillment of the terms of the Memorandum of Understanding (MOU) between the Hybrid Central Tracking Chamber (HCTC) collaboration and the SSC Laboratory for FY90. The report for FY1991 provides a comprehensive account of the research and development carried out under the MOU during the current fiscal year. Since most of the HCTC collaboration members are also members of the Solenoidal Detector Collaboration (SDC) and since our research is in direct support of the SDC detector, no funding request for FY1992 is included here; rather funds will be sought directly through SDC to continue the research and development efforts in future years. The HCTC collaboration has the same 12 member institutions as last year, with the cosmetic change that the group formerly from General Electric Canada, Inc. was acquired by EG\&G. The Executive Summary presents a brief overview of the HCTC design and the major accomplishments of FY1991. The remainder of this report consists of minireports from the member groups providing detailed information on their activities during the year. During FY1991, we have made considerable progress toward our goal of developing a large volume tracking detector for the SSC that barrows desirable features of two promising technologies, straw tubes and plastic scintillating fibers (PSF). Our research confirms our prior position that the hybrid straw-tube/PSF approach offers significant advantages in performance without compromising cost, permits straightforward and inexpensive upgrade to accommodate higher luminosity, and is a flexible option fo: sentral tracking the in the large general-purpose SDC detector.

Research sponsoreci by U.S. DOE and Superconducting SLper Collider Labordc:-:y.

*Duke University, Zi.urham, NC.

$\dagger^{\dagger}$ Instrumentation and Controls Division.

${ }^{\ddagger}$ MMES Engineering. 
4.35

\section{FEASIBILITY OF USING NEURAL NETWORKS AS \\ A LEVEL 2 CALORIMETER TRIGGER FOR JET TAGGING}

\author{
T. Handler* \\ E. Neis ${ }^{\dagger}$ \\ C. W. Glover T. A. Gabriel
}

\section{S. Saini}

(Abstract of paper presented at the Conference on Computing in High-Energy Physics, Annecy, France, September 21-25, 1992)

Several of the expected decay modes of the Higgs particle will result in jet formation. We pro pose to incorporate a second level trigger into the SDC detector. using neural network VSLl hardware, to tag such lliggs decay modes. The input to the neural network will be the energy depositions in both the barrel and endcap regions of the calorimeter. The neural networl's output would be a value representing the degree of correlation between the observed energy distribution and the type of physical scattering that has occurred. Preliminary results indicate that neural networks may be of use in tagging jet decays of the Higgs particle.

Research sponsured by L'.S. DOE and the Superconducting Super Collider Laboratory.

*University of 'Tcunnessee. Kinoxville, TN.

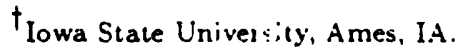

${ }^{\ddagger}$ Physics Division.

\subsection{6}

\section{COMPARISON OF CALOR89 MODEL PREDICTIONS WITH SCINTILLATOR PLATE CALORIMETER DATA}

\author{
P. K. Job* \\ J. Proudfoot* \\ L. E. Price* \\ B. L. Bishop ${ }^{\ddagger}$ \\ T. Handler ${ }^{\dagger}$ \\ T. A. Gabriel
}

[Abstract of Nucl. Instrum. Methods Phys. Res. A309, 60 (1991)]

The CALOR code system has been recently revised by adding a high energy collision model for energies $\geq 5 \mathrm{GeV}$. This version is called as
CALCR89. Calculations with this version have been carried out and compared with experimental data from four test beam scintillator plate calorimeter configurations. The agreement is good within the systematics. The limiting uncertainty in CALOR simulation at $10 \mathrm{GeV}$ is the choice of collision model. We have addressed this uncertainty by appealing to the experimental data and determined that by a careful transition from the Intranuclear Cascade model to he FLUKA model this can be reduced to $1.5 \%$. The other mostly mechanical effects such as cladding, scintillator saturation, shower integration time and shower containment can each produce systematic shifts as much as $4 \%$. However, careful modeling of the experimental configuration should allow the systematic effects from each of these to be minimized to $1 \%$. We find that an overall systematic uncertainty of $2.5 \%$ in the model predictions may then be achieved.

Research sponsored by U.S. DOE Superconducting Super Collider.

*Argonne National Laboratory, Argonne, IL.

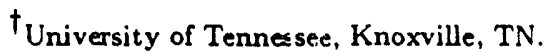

${ }^{\ddagger}$ Computing and Teiecrmmunications Division.

\subsection{7}

\section{DESIGN CONSIDERATIONS FOR A SCINTILLATING PLATE CALORIMETER}

\author{
P. K. Job* \\ L. E. Price* \\ J. Proudfoot* \\ T. Handler ${ }^{\dagger}$
}

T. A. Gabriel

[Abstract of Nucl. Instrum. Methods Phys. Res. A316,
i74 (1992)]

Results of the simulation studies for the design of a scintillating plate calorimeter for an SSC detector are presented. These simulation studies have been carried out with the CALOR89 code. The results show that both lead and uranium can yield good compensation in practical sampling geometries. However, the significant delayed energy release in the uranium systems can lead to a serious pile up problem at high rates. In the energy range under consideration, an ironscintillator system is not compensating at any absorber to scintillator ratio. An inhomogeneous 
calorimeter with $4 \lambda$ of lead-scintillator in a compensating configuration followed by $4 \lambda$ of ironscintillator with moderate sampling is found to perform as well as a homogeneous lead-scintillator compensating calorimeter. In such homogeneous systems the hadronic signal from different segments are weighted by a scheme based on minimum ionizing $d E / d X$. We show that, in a properly optimized three segment system. compensation and good hadronic resolution can be achieved by appropriately weighting the signal from the segments.

Research sponsored by (i.S. DOE and Superconducting S11per Collider Laburtlory:

*Argonne :iational laburatory: Argonne, Il,

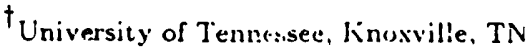

4.38

\section{SYSTEMATIC EFFECTS IN CALOR SIMULATION CODE TO MODEL EXPERIMENTAL CONFIGURATIONS}
P. K. Job*
J. Proudfoot*
T. Handler ${ }^{\dagger}$
T. A. Gabriel

(Abstract of paper presented al the Workshiop on Detector and Event Sinulation in High Energy Physics. Amsterdam, Netherlands, April 8-12,1901)

CALOR 89 code system is being used to simulate test beam results and the design parameters of several calorimeter configurations. It has been benchmarked against the ZEUS, DO and HELIOS data. This study identifies the systematic effects in CALOR simulation to model the experimental configurations. Five major systematic effects are identified. These are the choice of high energy nuclear collision model, material composition, scintillator saturation, shower integration time, and the shower containment. Quantitative estimates of these systematic effects are presented.

Research sponsured by LI.S. DOE and by the Office of Naval Kesearch.

* Argonne Natiunal Laboiatory, Argonne, IL.

†University of 'Temessec, Knovville. TN.
4.39

\section{SILICON ELECTROMAGNETIC CALORIMETER COLLABORATION PROGRESS REPORT}
H. W. Kraner*...
G. T. Alley ${ }^{\dagger}$
M. L. Bauer ${ }^{\dagger}$
C. L. Britton, $\mathrm{Jr}^{\dagger}$
H. R. Brashear ${ }^{\dagger}$
H. O. Cohn ${ }^{\ddagger}$
T. A. Gabriel
F. Plasil ${ }^{\ddagger}$
M. J. Rennich $\S$
R. A. Todd ${ }^{\dagger}$ et al.

(Abstract of ORNL/M-1776, September 1991)

The Silicon Electromagnetic Calorimeter Collaboration is working on seven tasks and substantial progress has been made on each of these tasks during the past year. A brief summary of the results by tasks is given. Some of these tasks include radiation hardness, electronic readout, mechanical engineering and simulation.

Research sponsored by the U.S. DOE and the Superconducting Super Collider Laboratory.

*Brookhaven National Laboratory, Upton, NY.

$\dagger^{\dagger}$ Instrumentation and Controls Division.

${ }^{\ddagger}$ Physics Division.

$\S_{\text {MMES Engineering. }}$

4.40

\section{RADIATION SHIELDING REQUIREMENTS FOR MANNED DEEP SPACE MISSIONS}

\section{R. T. Santoro \\ D. T. Ingersoll}

(Abstract of ORNL/TM-11808, April 1991)

Galactic cosmic rays (GCR) and, particularly, solar flares (SF) constitute the major radiation hazards in deep space. The dose to astronauts from these radiation sources and the shielding required to mitigate its effect during a $\mathbf{4 8 0}$ day Mars mission is estimated here for a simplistic spacecraft geometry. The intent is to "ball park" the magnitude of the doses for the constant GCR background and for SF's that occur randomly during the mission. The spacecraft shielding and dose data are given only for primary GCR and SF radiation, recognizing that secondary particles pro- 
duced by primary particle reactions in the spacecraft and High Z-High Energy particles will also contribute to the dose suffered by the astronauts.

Research sponsored by U.S. Department of Energy.

\subsection{1}

\section{LIQUID ARGON CALORIMETRY FOR THE SSC PROGRESS REPORT FOR FY 91 AND REQUEST FOR FUNDS FOR FY 92}

\author{
R. Steiner*... R. G. Alsmiller, Jr. \\ C. Y. Fu T. A. Gabriel, et al.
}

(Abstract of ORNL/M-1773, September 1991)

Our collaboration for research and development for liquid argon calorimetry (LAr) for the SSC has done a significant amount of work in the past year as outlined in this report and appendices. Our most coherent effort was a beam test of a hadronic calorimeter at Brookhaven National Laboratory followed by the addition of a separate electromagnetic (EM) module. This brought most of our collaborators together as well as providing a training ground for many outstanding graduate students and other young scientists. We also carried out work on a diverse array of related topics at our own institutions. Although the liquid argon technique is well established, the application of this technology to the SSC is very challenging and we must continue to do a significant amount of R\&D in FY1992. This may be thought of as having a direct connection with either the GEM or SDC experiments (which at this moment still both hold liquid argon as an important option). We must also point out that this collaboration has been able to work closely together even though we may be members of different experiments. We are especially grateful to the participation of our collaborators from Japan who took important responsibilities in the fabrication as well as operation of the beam tests. They also carried out a program of $R \& D$ in Japan that advanced our common goals.

Research sponsored by U.S. DOE and Superconducting Super Collider Laboratory.

* Adelphi University, Garden City, NY.
4.42

\section{SIMULATION OF A PRESAMPLER RESPONSE WITH CALOR - A COMPARISON WITH EXPERIMENTAL DATA}
C. Zeitnitz*
E. Fretwurst*
G. Lindström*
V. Riech*
T. A. Gabriel
T. Handler ${ }^{\dagger}$

(Abstract of DESY' 92-045, March, 199.2)

The reliable simulation of the detector response to high energetic particles plays a dominant role for the design of any large scale experiment in present and future colliders. Various Monte Carlo codes had beell especially tuned in order to match the experimental data obtained in calorimeters. Of main interest here is the visible energy, the energy resolution and the $\mathrm{e} / \mathrm{h}$ ratio. Although a very satisfying reproduction of known experimental results and therefore a high degree of reliability for calorimetric predictions could be achieved, there are various other subdetector components for which these codes are of much less accuracy. This is especially true for all applications in which the very first inelastic interactions of high energetic hadrons play an important role and hence the details of these reactions have to be reproduced accurately. An inportant example, where only the very first interactions with matter determine the response, is a preshower detector placed in front of a calorimeter. Such devices have become increasingly interesting for improved electron/hadron-separation and are presently discussed in various collaborations. The comparison between the experimental results obtained with a presampler and Monte (Carlo calculations are presented.

\footnotetext{
Kesearch sponsored by U.S. Department of Energy:

* Ciniversity of Hannberg. Cermusuy.

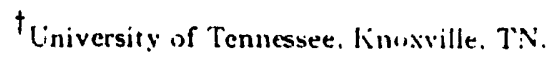




\section{DEFENSE APPLICATIONS}

\subsection{3}

\section{NUCLEAR VULNERABILITY OF THE U.S. M60A1 TANK IN AN INITIAL RADIATION ENVIRONMENT: MASH CODE SYSTEM ANALYSIS}

\author{
J. M. Barnes* \\ T. J. Burns \\ J. O. Johnson \\ J. D. Drischler
}

(Abstract of ORNL/TM-11775, May 1991)

The capability to accurately assess and predict the effectiveness of radiation shielding materials in vehicles, structures, trenches, and other configurations is of considerable interest to the DoD and the DNA. A research effort involving several institutions has worked towards providing this capability for several years, resulting in the first code system called the Vehicle Code System (VCS) and the successor to VCS, referred to as the Monte Carlo Adjoint Shielding Code system - MASH. The purpose of this report is to present the results of a re-assessment of the prompt nuclear weapon radiation shielding characteristics of the U.S. M60Al medium tank utilizing the MASH code system, and comparison to VCS results previously calculated and documented by BRL. Results of this comparison are to be used in the recommendations to the NATO Panel VII Ad Hoc Group of Shielding Experts for replacing VCS with MASH as the reference code of choice for future armored vehicle and other nuclear vulnerability calculations.

In general, the VCS and MASH neutron protection factor (NPF) results show a factor of 1.3 for the commander, 1.8 to 1.9 for the gunner, 1.6 to 1.7 for the loader, and approximately a factor of three for the driver ( 3.5 for VCS, and 2.8 for MASH). The gamma protection factor (GPF) results ranged from approximately 3 to 35 depending on crew member location, vehicle orientation, and source/vehicle ground range. Neutron protection factor changes versus vehicle range relative to point of burst are negligible, whereas variation of the vehicle orientation relative to the source can have a significant effect on both the neutron and gamma protection factors.

Research sponsored by the Defense Nuclear Agency.

*Computing and Telecommunications Division.
4.44

\section{DECAY OF GAMMA RADIATION IN FALLOUT FOLLOWING A NUCLEAR WAR}

\author{
C. M. Haaland \\ [Abstract of Health Physics (in press)]
}

An inportant factor that affects fallout casualty estimates is the normalization factor which indicates how much of the fallout is deposited lo cally. This factor was not mentioned in two recent papers (Harvey et al. 1992; Peterson and Shapiro 1992) in which fallout fatalities in a nuclear war were estimated. Furthermore, although ample evidence exists to sliow that the so-called "standard" $t^{-1.2}$ decay-law for gamma radiation in fallout from nuclear detonations does not agree with theory or actual data, this law was implicit in the fallout model used for these papers. If the results for fallout casualties in these papers were based on integrated dose resulting from computer simulated measurement.s of radiution rates at time ranging from $12 \mathrm{~h} \mathrm{to} 24 \mathrm{~h}$ after detonation, then the resultant integrated dose nationwide would probably be within $10 \%$ of result.s obtained by a more acceptable decay scheme. But if the results for fallout casualties were based on integrated doses resulting from assumed unit-time reference radiation rates, then the resultant estimate for integrated dose nationwide used in the cited papers could be a factor of two or more too high. These factors will not affect the principal conclusions of the studies because the promprt fatalities far outnumber the fallout fatalities for the scenarios chosun. However, for scenarios involving evacuation of population from high-risk areas, these factors could lower the estimale of fallout casualties by factors of two or more.

Research sponsurerl by U.S. Department of Energy. 
4.45

\section{PERTURBED ENVIRONMENT ASSESSMENTS FOR SPACE VEHICLES}

\section{M. Haaland}

(Abstract of ORNL/TM-11722, June 1991)

Three topics are discussed which are relevant to the technical assessment of the adequacy of environmental and response function analyses for space vehicles. First, a matrix is presented which summarizes exoatmospheric environmental modifiers and subsequent vehicular responses. Second, the early-time beta spectrum from a pulsed reactor experiment is compared with a beta spectrum which has been used for exoatmospheric analyses, showing that the average energy of the latter is significantly lower. Third, $x$-ray transport results are compared between ATR5 and XPRT5 for the high endoatmospheric regime (80 to 200 kilofeet altitude). Results are within less than $3 \%$ for altitudes for $150 \mathrm{kft}$ and above, where the buildup factors are low, but differences up to $43 \%$ occur at lower altitudes. Possible reasons for these discrepancies are discussed.

Research sponsored by Strategic Defense Commind.

4.46

\section{CALCULATION OF PROTECTION FACTORS OF THE M1A1 TANK AGAINST INITIAL NUCLEAR RADIATION}
C. M. Haaland
J. O. Johnson
R. T. Santoro
K. G. Kerris*

(Abstract of paper presented at the Third Annual Combat Vehicle Survivability Symposium, Gaithersburg, MD, March 31-April 2, 1992)

The Monte Carlo Adjoint Shielding (MASH) code system was used to calculate protection fis:tors (PFs) for various electronic components and crew of the MlAl (Heavy) Abrams tank. The calculations used a combinatorial geometry representation of the talk prepared by Ballistic Research Laboratories (BRL) consisting of $7850+$ bodies with $6200+$ regions. PFs for electronic components were found for initial nuclear radiation (INR) from a $3 \mathrm{kT}$ boosted-fission tactical weapon for comparison with earlier computations by Romanelli, et al. PFs for the crew ware :al. culated for the standard configurations aril pro- cedures as specified by AEP-14 ${ }^{2}$, with a fission source based on the NATO standard reference environment at $400 \mathrm{~m}$ from the Army Pulsed Reactor Facility; Aherdeen Proving Grounds, MD. Comparisons were made to assess the different effects on nuclear shielding between conventional and special armor.

Research sponsored by I:S. DOE and by U.S. Army LABCOM. Harry I Diamond laboratories.

*U.S. Army LABCOM. Harry Diamond Laboralories Adelphi, MD

\subsection{7}

\section{NUCLEAR VULNERABILITY OF THE U.S. M60A1 TANK IN AN INITIAL RADIATION ENVIRONMENT: MASH CODE SYSTEM ANALYSIS}
J. O. Johnson
T. J. Burns
J. D. Drischler
J. M. Barnes*

[Abstract of paper presented at the American Nuclear Society Topical Meeting on New Horizons in Radiation Protection and Shielding, Pasco, WA, April 26-30 (1992); Proc. pp. 267-274 (1992)]

The capability to accurately assess and predict the effectiveness of radiation shielding materials in tactical armored vehicles is of considerable interest to the Department of Defense (DoD). This report presents the results of a reassessment of the prompt nuclear weapon radiation shielding characteristics of the U.S. M60A1 medium tank utilizing the Monte Carlo Adjoint Shielding Code system - MASH, and comparisons to the Vehicle Code System (VCS) results previously calculated and documented by Ballistic Research Laboratory (BRL).

Analysis of the neutron and gamma doses and protection factors indicate excellent agreement between VCS and MASH. The VCS and MASH neutron protection factors (NPFs) are approximately 1.3 for the commander, 1.9 for the gunner, 1.7 for the loader, and 3.1 for the driver. The gamma protection factors (GPFs) range from approximately 3 to 35 depending on crew member location, vehicle orientation, and source/vehicle ground range. Neutron protection factor changes versus vehicle range relative to point of burst are negligible, whereas variation of the vehicle orientation relative to the source can have a significant effect on both the neutron and gamma protection factors.

Research sponsored by U.S. DOE Defense Nuclear Agency.

*Computing and Telecommunications Division. 
4.48

\section{ANALYSIS OF THE FALL-1989 TWO-METER BOX TEST BED EXPERIMENTS PERFORMED AT THE ARMY PULSE RADIATION FACILITY (APRF)}

\author{
J. O. Johnson J. D. Drischler \\ J. M. Barnes*
}

(Abstract of paper presented at the American Nuclear Society Topical Meeting on New Horizons in Radiation Protec. tion and Shielding, Pasco, WA. April 26-30 (1 192$)$; Proc. pp. 251-258 (1992); also ORNL/TM-11777, May 1991]

MASH, a Monte Carlo Adjoint Shielding Code system, was developed to provide the capability of accurately assessing and predicting the effectiveness of radiation shielding materials in vehicles, structures, trenches, and other configurations of interest to the Department of Defense (DoD). This paper presents the results of a "benchmark" analysis of MASH against a series of experiments performed at the Army Pulse Radiation Facility (APRF) and its capability of reproducing the measured neutron and gamma-ray integral and spectral data. Free-field environments at 170 and 400 meters and "in-box" environments were measured and calculated using the "North Atlantic Treaty Organization (NATO) standard test bed," i.e., the two-meter box, at the "NATO standard reference point" at 400 meters.

The calculation to experiment $(C / E)$ ratios of the free-field and in-box neutron tissue dose indicate consistent agreement at the 170 and 400 meter test sites with typical agreement within $10 \%$ at 170 meters and between $10 \%$ and $20 \%$ at the 400 meter test site. For gamma rays, the $\mathrm{C} / \mathrm{E}$ ratios indicate the calculations underestimate the measurements by approximately $35 \%$ at 170 meters, $25 \%$ at 400 meters, and approximately $10 \%$ inside the two-meter box. The fluence spectra comparisons support the results obtained in the integral data comparisons. The comparisons of the reduction factor results show $\mathrm{C} / \mathrm{E}$ ratios ranging from 1.22 to 0.76 with most of the $C$. $E$ ratios between 1.07 and 0.80 .

Research sponsored by U.S. DOE Defense Nucleas Agency.

*Computing and Telecommunjcations Division.

4.49

\section{ANALYSIS OF THE SPRING-1990 TWO-METER BOX TEST BED EXPERIMENTS PERFORMED AT THE ARMY PULSE RADIATION FACILITY (APRF)}

\author{
J. O. Johnson J. D. Drischler \\ J. M. Barnes*
}

(Abstract of ORNL/TM-11917, August 1992)

The capability to accurately assess and predict the effectiveness of radiation shielding materials in vehicles, structures, trenches, and other configurations is of considerable interest to the DoD and the DNA. A research effort involving several institutions has worked towards providing this capability for several years, resulting in the Monte Carlo Adjoint Shielding Code system - MASH. The purpose of this report is to present the results of a "benchmark" analysis of MASH against a set of measurements performed in the Spring of 1990 at the APRF and determine the capability of MASH in reproducing the measured neutron and gamma-ray dose data. In particular, the free-field environment was calculated along with measurements made using the "NATO standard test bed" (i.e. a two-meter box) and the RT-200 anthropormorphic phantom standing in the free-field and inside the two-meter box at the "NATO standard reference point" at 400 meters.

The calculational results show mixed agreement with the measured data reported by the different teams of experimentalist. The neutron dose results typically agreed for the phantom standing in the free-field but consistently under-estimated the measured dose for the phantorn standing inside the two-meter box. The neutron reduction factors also agreed for the phantom standing in the free-field but exhibited significant differences for the phantom standing inside the box. With respect to the gamma-ray data, there were individual discrepancies between calculations and measurements however, typical agreement was within $\pm 20 \%$. The gamma-ray free-ficld environment at 400 meters continues to show a discrepancy between calculation and experiment which further manifest itself through the omparisons of the gamma-ray reduction factors.

To determine the overall quality of the comparisons between MASH and the measurements, 
the data (both calculational and experimental) were averaged over detector position (internal and external), response function (Tissue and $\mathrm{CaF}_{2}: \mathrm{Mn}$ ), radiation type (neutron and gammaray), and phantom orientation (front facing and right side toward reactor). This averaging procedure produced one set of data for the phantom in the free-field, and one set of data for the phantom inside the two-meter box. In general, the calculations agreed quite well with most of the measured data supplied by the experimental teams. The agreement was within the $\pm 20 \%$ limit deemed as acceptable by the DNA.

Research sponsored by U.S. DOE and Defense Nuclear Agency.

* Computing and Telecommunications Division.

\subsection{0}

\section{ANALYSIS OF THE SPRING-1991 TWO-METER BOX TEST BED EXPERIMENTS PERFORMED AT THE ARMY PULSE RADIATION FACILITY (APRF)}

\author{
J. O. Johnson J. D. Drischler \\ J. M. Barnes*
}

(Abstract of ORNL/TM-11918, September 1992)

The capability to accurately assess and predict the effectiveness of radiation shielding materials in vehicles, structures, trenches, and other configurations is of considerable interest to the DoD and the DNA. A research effort involving several institutions has worked towards providing this capability for several years, resulting in the Monte Carlo Adjoint Shielding Code System - MASH. The purpose of this report is to present the results of a "benchmark" analysis of MASH against a third set of measurements performed in the Spring of 1991 at the APRF and determine the capability of MASH in reproducing the nieasured neutron and gamma-ray integral data. In particular, the free-field environment was calculated along with measurements made using the "NATO standard test bed" (i.e. a two-meter box) and the RT-200 anthropormorphic phantom stauding in the freefield and inside the two meter box lined with $5 \%$ borated polyethylene at the "NATO standard reference point" at 400 meters.
The calculational results show mixed agreement with the measured data reported by the different teams of experimentalists. The neutron dose results indicate good agreement (typically $\pm 20 \%$ ) for the phantom standing in the free-field and excellent agreement (typically $\pm 10 \%$ ) for the phantom standing inside the two-meter hox. The neutron reduction factors however, agreed for the phantom standing in the free-field but exhibited significant differences for the phant om st anding inside the hox. With respect to the garmunit-ray data, there were individual discrepancies betweell calculations and measureme!ts: lonwever. typical agreement was within $\pm 20 \%$. To detrrmine the overall quality of the comparisons between MASH and the measurements, the data was averaged over detector position response function, radiation type, and phantom orientation. This averaging procedure produced one set of data for the phantorn standing in the free-field, and one set of data for the phantom standing inside the lined two-meter box. In general, the calculations agreed quite well with most of the measured data supplied by the teams of experimentalists. The agreement was within the $\pm 20 \%$ limit deemed as acceptable by the DNA.

Research sponsored by Defense Nuclear Agency and U.S. DOE.

* Computing and Telessmmunications Division.

\subsection{1}

\section{ANALYTIC METHODS FOR RADIATION ENVIRONMENT SIMULATION AND VULNERABILITY ASSESSMENT}
J. O. Johnson
R. T. Santoro
T. J. Burns
R. A. Kehlet*

(Abstract of paper presented at the Test Technology Symposium V, John Hopkins University, Applied Physics Laboratory, Laurel, MD, July 14-16, 1992)

The capability of armor/structural materials in protecting the occupants and equipment in tactical vehicles or other shielded structures against nuclear weapon radiation will remain a continuing problem for military system designers. Since there is no procedure for measuring radiation effects in realistic weapon radiation environments, acquisition of radiation protection factors must come from measurements at reactor sites (e.g., 
Army Pulse Radiation Facility) or from detailed radiation transport calculations.

The Monte Carlo Adjoint Shielding code system - (MASH), has been developed to calculate neutron and gamma-ray environments and radiation protection factors for armored military vehicles, and other shielding configurations. The shielding effectiveness can be characterized for both personnel and electronic equipment as a function of weapon height, source/target ground range, and vehicle orientation and configuratioa. Comprehensive comparisons between measured data obtained at APRF and calculated results using MASH have been made for a variety of shielded systems including the M60 and M1A1 tanks and the Soviet BMP tactical infantry vehicle. The performance of MASH has been demonstrated with acceptable confidence so that radiation analysis and nuclear performance data can be straightforwardly calculated thereby eliminating expensive field measurements.

One recurring difficulty in radiation analyses is the construction and verification of the geometry model that represents the actual physical configuration to be analyzed. Currently, the geometry models required by MASH are laboriously translated from blueprints or other descriptions into combinatorial geometries by specifying both the fundamental objects (spheres, cylinders, cones, etc.) as well as the Boolean operations required to combine the objects into meaningful assemblies. To alleviate this difficulty, a graphical debugger for combinatorial geometry input to MASH is being developed at the Oak Ridge National Laboratory. The prototype debugger consists of two parts: a FORTRAN-based "view" generator and a Microsoft Windows application for displaying the geometry. The debugger can also be used as a visualization tool for the output of the radiation transport codes. Using one of the output options of MASH, graphical displays of "weak" spots in vehicle/structure shielding can be readily identified thereby permitting designers to appropriately place shielding to protect personnel and equipment.

Research sponsored by Defense Nuclear Agency and U.S. Department of Energy.

*Defense Nuclear Agency, Alexandria, VA.
4.52

\section{RADIATION PROTECTION FOR HUMAN EXPLORA'TION OF THE MOON AND MARS: APPLICATION OF THE MASH CODE SYSTEM}
J. O. Johnson
R. T. Santoro
J. D. Drischler
J. M. Barnes*

IAbstract of paper presented at the American Nuclear Society Topical Meeting on Nuclear Technologies for Space Exploration, Jackson Hole, WY, August 16-19, 1992; Proc. Vol. III, pp. 697-706 (1992)]

The Monte Carlo Adjoint Shielding code system-MASH, developed for the Department of Defense for calculating radiation protection factors for armored vehicles against neutron and gamma radiation, has been used to assess the dose from reactor radiation to an occupant in a habitat on Mars. The capability of MASH to reproduce measured data is summarized to demonstrate the accuracy of the code. The estimation of the radiation environment in an idealized reactor-habitat model is reported to illustrate the merits of the adjoint Monte Carlo procedure for space related studies. The reactor radiation dose for different reactor-habitat-surface configurations to a habitat occupant is compared with the natural radiation dose acquired during a 500-day Mars mission.

Research sponsored by U.S. DOE Defense Nuclear Agency.

* Computing and Telecommunications Division.

\subsection{3}

\section{RADIATION EXPOSURE INSIDF REINFORCED CONCRETE BUILDINGS AT NAGASAKI}

\author{
W. A. Rhoades R. L. Childs* \\ D. T. Ingersoll
}

[Abstract of Health Physics 63(3), 510 (1992)]

In this study, the radiation doses to occupants of two reinforced concrete buildings at Nagasaki who survived the immediate effects of the nuclear weapon detonation are determined using stateof-the-art radiation transport techniques. The radiation doses at all locations in the buildings are calculated using the Three Dimensional Oak Ridge Discrete Ordinates Radiation Transport Code (TORT) which was constructed especially 
for this task. TORT represents a new and unique capability that has been reported elsewhere. This study resulted in case-by-case lists of doses to occupants and an uncertainty analysis. These data have been used in : companion study as the basis for determining a new value of the LD50 parameter:

Research sponsored by Defense Nuclear Agency.

* Computing and Teleconumunications Division.

\subsection{4}

TRANSMISSION FACTCES FOR THF PENETRATION OF NEUTRON AND PHOTON FLUENCE INTO WOOD-FRAME DWELLINGS, 1990 (TF90)

\author{
W. A. Rhoades R. A. Lillie \\ M. B. Emmett ${ }^{*}$ \\ (Abstract of ORNL/TM-12021, May 1992)
}

The "DS86" study conducted at several laboratories in the United States and Japan calculated doses to persons inside wood-frame dwellings at both Nagasaki and Hiroshima. While it is not practival to determine an individual nuclear model for each case in such a large data base, the study found that the construction of divellings in those cities was fairly standardized, and that the radiation environment could be adequately modeled by a small number of typical buildings.

The study was successful in applying its methodology to the data base maintained by the Radiation Effects Research Foundation (RERF) in Japan. The numerical results were embodied in a complex computer-based process that was tied to the specific structurr. of the RERF data base, however, and attempts $\omega$ apply them to the data base maintained by the Dikewood Division, Kaman Sciences, in Albuquerque, $\mathrm{NM}$ have been deemed technically unsatisfactory. The present study is tirected toward applying the general technical approach of the DS86 study in a way that is applicable to the Dikewood data base.

Briefly, the method began with the calculation of radiation fluence at several ground ranges spanning the locations of the dwelling sites. Then, the effectiveness of fluence at the surface of a box enclosing the neighborhood in penetrating to individual sites within a cluster of typical dwellings was calculated using an adjoint Monte Carlo method.

By folding this adjoint result with the fluence data exterior to the dwelling cluster, the radiation at interior sites were determined. By moving the cluster to different ranges and rotating it with respect to the source, various radiation environments were simulated. By using the proper Monte Carlo response spectrum, free-in-air kerma (FIA), average small intestine kerma (SI), or average bone marrow kerma (BM) could be calculated. The transmission factor (TF) was obtained by dividing interior kerma by external kerma at a given site.

F Inally, individual results were identified with combinations of parameters encoded in the Dikewood data files, and results with like parameters were "binned" together.

The doses corresponding to a specific medical case were then determined by finding the exterior FIA doses, and then multiplying by the transmission factors from the table position having corresponding Dikewood parameters. The TF data were interpolated between the ground ranges in the table. The three response types, i.e. FIA, SI, or BM, were obtained from the corresponding data in the tables.

Research sponsored by U.S. DOE and Defense Nuclear Agency.

* Computing and Telecommunications Division.

4.55

\section{DNA RADIATION ENVIRONMENTS PROGRAM FALL 1989 2-METER BOX EXPERIMENTS AND ANALYSIS}

\section{R. T. Santoro}

(Abstract, of ORNL/TM-11840, May 1991)

This report summarizes the Fall 1989 2-m Box Experiment Performed at the Army Pulse Reactor Facility (APRF) at Aberdeen Proving Ground. This effort, sponsored by the Defense Nuclear Agency under the Radiation Environments Program, was carried out to obtain measured data for benchmarking MASH, the Monte Carlo Adjoint Code System. MASB was developed to replace the Vehicle Code System, VCS, that has been used by the Department of Defense and NATO for cal- 
culating neutron and gamma-ray radiation fields and shielding protection factors inside armored vehicles and structures from nuclear weapon radiation. Measurements of the free-field differential spectra and kerma were performed by experimentalists from the APRF, the Defense Research Establishment Ottawa, Canada and the Establissement Technique Central de l'Armement, France. Free-field data svere obtained at distances of 170and 400-meters from the APR while in-box measurements were made at 400 meters only. The box, included to obtain neutron and gamma-ray reduction factors, was a 2-meter cube configuration having 0.1 U16-m-thick steel walls. Calculated data were obtained using MASH by analysts from the Oak Ridge National Laboratory and Science Applications International Corporation. Calculated (C) results were compared with experimental (E) data in terms of $\mathrm{C} / \mathrm{E}$ ratios. The Defense Nuclear Agency, with concurrence of the program participants, established $\pm 20 \%$ as the acceptable C/E for this study and for qualifying MASH.

Free-field and in-box neutron kerma generally agreed within $\pm 20 \%$, although some $\mathrm{C} / \mathrm{E}$ comparisons fell outside this range depending upon the detector against which the calculated data were compared. For those cases where the $\mathrm{C} / \mathrm{E}$ ratio is marginal or unacceptable, problems in the detector systems were acknowledged to be the principal cause of the discrepancy. Generally poor agreement ( 25-35\%) was achieved among the C/E ratios for the free-field gamma-ray Kerma at the 170 - and $400-m$ locations while excellent $(10 \%$, or better) $C / E$ values were obtained for the in-box conditions. The discrepancy for the free-field comparison was attributed to the failure by the analysts to include a tree line adjacent to the measurement site in the calculational geometry. $\mathrm{C} / \mathrm{E}$ values for the neutron and gamma-ray reduction factors ranged from $1 \%$ to $23 \%$ depending on the detector. Comparisons of calculated with the measured neutron and gamma-ray differential spectra ranged from excellent to marginal depending on choice of detector and energy interval.

Resiearch sponsored by the Defense Nuclear Agency.

4.56

\section{RESOLUTION OF DOSE AND REDUCTION FACTOR QUESTIONS IN ARMY PULSE RADIATION FACILITY EXPERIMENTS: RENORMALIZATION OF CALCULATED AND MEASURED DATA}

\author{
R. T. Santoro J. D. Drischler
}

J. O. Johnson

(Abstract of ORNL/TM-12148, November 1992)

A procedure is introduced to normalize calculated and measured dose and reduction factors for a series of experiments performed at the Army Pulse Radiation Facility. In an attempt to isolate causes of the differences among these ciata, the data are reevaluated on the basis that the free-field neutron and gamma-ray fluence at $400 \mathrm{~m}$ from the reactor should be constant within air and ground moisture conditions. When the dose data are compared relative to the number of free-field neutrons, significant improvements are realized in the calculated-to-measured data ratios. Discrepancies in previously reported results appear to be traceable to differences in the number of free-field neutrons and the $\gamma / \mathrm{n}$ ratio at the measurement location.

Research sponsored by Defense Nuciear Agency ard U.S. DOE.

\subsection{7}

DNA RADIATION ENVIRONMENTS

PROGRAM SPRING 1990 2-METER BOX EXPERIMENTS AND ANALYSES
R. T. Santoro
S. Y. Whitaker*

(Abstract of ORNL/TM-12160, September 1992)

This report summarizes the Spring 1990 2-m Box Experiments performed at the Army Pulse Radiation Facility (APRF) at Aberdeen Proving Ground, Maryland. These studies were sponsored by the Defense Nuclear Agency (DNA) under the Radiation Environments Program to obtain measured data for benchmarking the Adjoint Mionte Carlo Code System, MASH, Version 1.0. 
MASH was developed as the Department of Defense and NATO code system for calculating neutron and gamma-ray radiation fields and shielding protection factors for armored vehicles and military structures against nuclear weapon radiation. In the experiments, neutron and gamma-ray dose and reduction factors were measured in the freefield and as a function of position on an anthropomorphic phantom that was placed outside and inside the steel-walled 2-m box. The data were acquired at a distance of $400-\mathrm{m}$ from the APRF reactor. The measurements were performed by APRF Bubble Technology Industries, the Defense Research Establishment Ottawa, Etablissment Technique Central de l'Armement, and Harry Diamond Laboratory. Calculations were carried out by the Oak Ridge National Laboratory and Science Applications International Corporation.

The purpose of these experiments was to measure the neutron and gamma-ray dose as a function of detector location on the phantom for cases when the phantom was standing in the free-field and inside of the box. Neutron measurements were made using a BD-100R bubble detector and gamma-ray measurements were made using thermoluminescent detectors (TLD). Calculated and measured data were compared in terms of the $\mathrm{C} / \mathrm{M}$ ratio. DNA mandated that $\mathrm{C} / \mathrm{M}$ values of $\pm 20 \%$ define the acceptable limits for the comparison of the dose and reduction factor data and for qualifying the MASH code in replicating integral parameters.
Neutron measurements were made only by Bubble Technology Industries while gamma-ray measurements were made by all of the teams of experimentalists. The measured gamma-ray doses exhibited considerable spread and $\mathrm{C} / \mathrm{M}$ ratios often exceeded $20 \%$. The calculated gamma-ray data reported by ORNL and SAIC were consistently in ayreement. The measured gamma-ray doses as a function of detector location varied significantly but when the average measured and calculated doses and reduction factors were compared, the $\mathrm{C} / \mathrm{M}$ ratio was within $\pm 20 \%$. The neutron doses and reduction factors showed a similar behavior but, on the average, agreement between the measured and calculated data was within the accepted tolerance. In those cases where large discrepancies occurred in the compaisons of reduction factors, the differences were traceable to disagreement in the measured and calculated freefield doses.

Accepting the $\mathrm{C} / \mathrm{M} \leq 20 \%$ criterion and the consequences of this study, it is recommended that MASH Version 1.0 be adopted by the DOD and NATO for calculating neutron and gamma-ray doses and reduction factors for armored vehicles and other shielded structures.

Research sponsored by Defense Nuclear Agency and U.S. DOE.

* Clark Atlanta University, Atlanta, GA.

\section{GENERAL METHODS DEVELOPMENT}

\subsection{8}

\section{A COMPARISON BETWEEN THE FINI'TE DIFFERENCE AND NODAL INTEGRAL METHODS FOR THE NEUTRON DIFFUSION EQUATION}

\section{Y. Y. Azmy}

A Abstract of paper presented at the American Nuclear Society Topical Meeting on Advances in Mathematics, Computation and Reactor Physics, Pittsburgh, PA, April 28-May 1, 1991; Proc. Vol. 4, p. 16.1, 2-1 (1991)]

The lowest order Nodal Integral Method (NIM) which belongs to a larger class of nodal methods, the Lawrence-Dorning class, is written in a five-point, weighted-difference form and contrasted against the edge-centered Finite Difference Method (FDM). The final equations for the two methods exhibit three differences: the NIM employs almost three times as many discrete-variable (which are node- and surface-averaged values of the flux) as the FDM; the spatial weights in the NIM include hyperbolic functions opposed to the algebraic weights in the FDM; the NIM explicitly imposes continuity of the net current across cell edges. A homogeneous model problem is devised to enable an analytical study of the numerical solutions accuracy. The analysis shows that 
on a given mesh the FDM calculated fundamental model eigenvalue is more accurate than that calculated by the NIM. However, the NIM calculated flux distribution is more accurate, especially when the problem size is several times as thick as the diffusion length. Numerical results for a nonhomogeneous test problem indicate the very high accuracy of the NIM for fixed source problems in such cases.

Research sponsored by U.S. Department of Energy.

\subsection{9}

\section{A NODAL INTEGRAL METHOD FOR NEUTRON DIFFUSION IN HEXAGONAL GEOMETRY}

\section{Y. Y. Azmy}

[Abstract of paper presented at the American Nuclear Society Topical Meeting on Advances in Reactor Physics, Charleston, SC, March 8-11, 1992; Proc. Vol. 1, pp. 509$519(1992)]$

A nodal integral method is derived for the monoenergetic, steady-state, fixed source neutron diffusion equation in hexagonal geometry based on a coordinate transformation that maps a parallelogram into a rectangle. The new Hexagonal Nodal Diffusion method is implemented in the computer code HND where the discrete-variable equations are solved via an iterative scheme. Because the new method's equations are derived for a rhombus, they can be solved on a sequence of embedded meshes to study the method's error order. Indeed a preliminary numerical error analysis reveals a second-order error in the mesh size, and comparison with finite difference results obtained with the finite difference based BOLD-VENTURE code indicate the superior accuracy of our new nodal method.

Research sponsored by U.S. Department of Energy.
4.60

\section{ARBITRARILY HIGH ORDER CHARACTERISTIC METHODS FOR SOLVING THE NEUTRON TRANSPORT EQUATION}

\author{
Y. Y. Azmy \\ [Abstract of Annals of Nuclear Energy 19, 593 (1992)]
}

We derive an arbitrarily high order characteristic method and describe its implementation in an Arbitrarily High Order Transport (AHOT) code, together with a previously derived nodal method. We test the accuracy and computational efficiency of the implemented methods by solving a one group homogeneous problem with three values of the scattering ratio, and a three group, three region shielding problem. We find that the nodal method is more accurate in computing quantities related to the scalar flux, while the characteristic method computes quantities strongly influenced by neutron streaming more accurately. The difference in accuracy between the two methods diminishes as the scattering ratio increases. Also, by monitoring the number of negative cell-averaged scalar fluxes as a fraction of the total number of computational cells in each case we find that even order methods are far less likely to produce negative fluxes. Furthermore, we observe that an even order characteristic method is less susceptible to converging negative cell-averaged scalar fluxes than the same order nodal method.

Research sponsored by U.S. Department of Energy.

\subsection{1}

GENERAL ORDER NODAL TRANSPORT METHODS AND APPLICATION TO PARALLEL COMPUTING

\section{Y. Y. Azmy}

[Abstract of Transport Theory and Statistical Physics (in press)]

Highly accurate solutions for neutron transport problems are achievable using high order nodal methods, such as the General Order Nodal Transport (GONT) class of methods. The final equations for these methods constitute a set 
of Weighted Diamond-Difference (WDD) equations that are solved using standard mesh sweeps. A parallel algorithm for solving these equations, based on decomposition of the angular domain is particularly suited for message-passing computers as it embodies a statically-scheduled, coarsegrained parallelization. The parallel code, PGONT implemented on Intel's iPSC/ 2 hypercube produces large speedup factors at very high parallel efficiencies. A mathematical model for execution time as a function of the problem parameters: spatial approximation order, number of mesh cells, and angular quadrature order, as well as the number of utilized processors, agrees very well with measured results. The model shows that the parallel efficiency is insensitive to the number of mesh cells, but improves with the spatial approximation and angular quadrature orders. It also shows that the speedup factor increases monotonically with the number of utilized processors, if the latter divides exactly the number of independent processes.

Research sponsored by U.S. Department of Energy.

\subsection{2}

\section{PERFORMANCE AND PERFORMANCE MODELING OF A PARALLEL ALGORITHM FOR SOLVING THE NEUTRON TRANSPORT EQUATION}

\author{
Y. Y. Azmy
}

[Abstract of Journal of Supercomputing 6, 211 (1992)]

A parallel algorithm is derived for solving the discrete-ordinates approximation of the neutron transport equation, based on the naturally occurring decoupling between the mesh sweeps in the various discrete directions during each iteration. In addition, the parallel Source Iteration (SI) algorithm, characterized by its coarse granularity and static scheduling, is implemented for the Nodal Integral Method (NIM) into the Parellel Nodal Transport (P-NT) code on Intel's iPSC/2 hypercube. Measured parallel performance for solutions of two test problems is used as evidence of the parallel algorithm's potential for high speedup and efficiency. The measured performance data is also used to develop and validate a parallel performance model for the total, serial, parallel, and global-summation time components per iter- ation as a function of the spatial mesh size, the problem size (number of mesh cells and angular quadrature order), and the number of utilized processors. The potential for high performance (large speedup at high efficiency) for large problems is explored using the performance model, and it is concluded that present applications in threedimensional Cartesian geometry will benefit by concurrent execution on parallel computers with up to a few hundred processors.

Research sponsored by U.S. Department of Energy.

\subsection{3}

\section{GENERAL ORDER CHARACTERISTIC METHODS FOR SOLVING NEUTRON TRANSPORT PROBLEMS}

\author{
Y. Y. Azmy
}

[Abstract of paper presented at the International Symposium on Numerical Transport Theory, Moscow, Russia. May 23-31, 1992; Proc. p. 49 (1992)]

The neutron transport equation in Cartesian geometry possesses straight line characteristics along which the streaming operator can be written as a full differential in terms of the characteristic length. This idea was used by Lathrop to develop the step characteristic method, which he showed to be positive definite but less accurate than conventional Diamond-Difference schemes. Several authors since then have developed new methods utilizing the characteristic curves (including nonCartesian geometry). A Linear Characteristic Method, based on a more consistent linear representation of the incoming-surface and within-cell angular flux, has been developed and tested in two-dimensional geometry producing highly accurate and computationally efficient results. A similar linear method, with several modifications, was developed for three-dimensional Cartesian geometry, and implemented in ORNL's production code TORT. In this paper we present a fully consistent, two-dimensional Cartesian geometry, general order characteristic method, in the same spirit as the previously developed, general order nodal method. Preliminary tests and numerical error analysis of the new method for orders up to five are also presented.

Research sponsored by U.S. Department of Energy. 


\subsection{4}

GRAPHICAL-BASED CONSTRUCTION OF COMBINATORIAL GEOMETRIES

FOR RADIATION TRANSPORT AND SHIELDING APPLICATIONS

\section{T. J. Burns}

(Abstract of paper presented at the American Nuclear Society Topical Meeting on Nuclear Technologies for Space Exploration, Jackson Hole, WY, August 16-19, 1992; Proc. Vol. III, pp. 717-727 (1992)]

A graphical-based code system is being developed at ORNL to manipulate combinatorial geometries for radiation transport and shielding applications. The current version (basically a combinatorial geometry debugger) consists of two parts: a FORTRAN-based "view" generator and a Microsoft Windows application for displaying the geometry. Options and features of both modules are discussed. Examples illustrating the various options available are presented. The potential for utilizing the images produced using the debugger as a visualization tool for the output of the radiation transport codes is discussed as is the future direction of the development.

Research sponsored by U.S. DOE Defense Nuclear Agency.

\subsection{5}

\section{CGVIEW - A PROGRAM TO GENERATE ISOMETRIC AND PERSPECTIVE VIEWS OF COMBINATORIAL GEOMETRIES}

\section{T. J. Burns}

(Abstract of ORNL/TM-12019, July 1992)

The prototype of a graphical debugger for combinatorial geometry (CG) is described. The prototype debugger consists of two parts: a FORTRAN-based "view" generator and a Microsoft Windows application for displaying the geometry. This document describes the code CGVIEW, which comprises the first part of the system. User-specified options permit the selection of an arbitrary viewpoint in space and the generation of either an isometric or perspective view. Additionally, any combination of zones, materials, or regions can be flagged as invisible to facilitate the inspection of internal details of the geometry. In the same manner, an arbitrary body can be cut away from the geometry to facilitate inspection and debugging. Examples illustrating the various options are described.

Research sponsored by U.S. DOE and Defense Nuclear Agency.

4.66

\section{GRAPHICAL DEBUGGING OF COMBINATORIAL GEOMETRY}

\section{T. J. Burns M. S. Smith}

[Abstract of paper presented at the American Nuclear Society Topical Meeting on New Horizons in Radiation Protection and Shielding, Pasco, WA, April 26-30, 1992; Proc. pp. 48-55 (1992)]

A graphical debugger for combinatorial geometry being developed at Oak Ridge National Laboratory is described. The prototype debugger consists of two parts: a FORTRAN-based "view" generator and a Microsoft Windows application for displaying the geometry. Options and features of both modules are discussed. Examples illustrating the various options available are presented. The potential for utilizing the images produced using the debugger as a visualization tool for the output of the radiation transport codes is discussed as is the future direction of the development.

Research sponsored by U.S. DOE Defense Nuclear Agency.

\subsection{7}

\section{THE WEIGHTED-DIFFERENCE FORM OF THE NODAL DIFFUSION METHOD IN CYLINDRICAL GEOMETRY}

\author{
L. Deleanu* Y. Y. Azmy \\ R. T. Primm, III
}

[Abstract of paper presented at the American Nuclear Society Topical Meeting on Advances in Mathematics, Computation and Reactor Physics, Pittsburgh, PA, April 28-May 1, 1991; Proc. Vol. 4, p. 16.1, 3-1 (1991)]

A nodal integral method is derived for the neutron diffusion equation in cylindrical, $r-z$ geometry. The final discrete-variable equations that express nodal balance of neutrons and net current continuity across node boundaries, are case in a five-point, weighted-difference form. These 
equations are identical in form, but with different expressions for the spatial weights, to their counterparts in cartesian geometry. Hence, the iterative procedure previously developed for the latter, is used to solve our newly installed $r-z$ nodal method in the production code BOLDVENTURE. Preliminary numerical experiments on simple one and two group, fixed source, test problems demonstrate the superior accuracy of the nodal method compared to the finite difference method.

Research sponsored by U.S. Department of Energy.

* Computing and Telecommunications Division.

\subsection{8}

THE CELL ANALYTICAL-
NUMERICAL (CAN) METHOD
FOR THE SOLUTION OF THE
RICHARDS EQUATION

\section{O. A. Elnawawy* Y. Y. Azmy}

[Abstract of paper presented at the IX International Conference on Computational Methods in Water Resources, Denver, CO, June 9-12, 1992; Proc. Vol. 1, p. 203 (1992)]

In a previous study, the CAN method has been developed and its performance evaluated for the solution of multidimensional groundwater solute transport problems in the saturated zone, which are linear in nature. The CAN method is an extension of the nodal method developed in the nuclear engineering field. In this study, we develop and describe implementation of the CAN method for solving the time-dependent, one-dimensional water flow problem in the unsaturated zone. This problem is characterized by the highly nonlinear nature of the governing equation, the Richards' equation.

Research sponsored by U.S. Department of Energy.

*Purdue University School of Science at Indianapolis, Indianapolis, IN.
4.69

PARALLELIZATION OF A
SPHERICAL $S_{N}$ ALGORITHM
BASED ON THE SPATIAL
DOMAIN DECOMPOSITION

A. Haghighat* Y. Y. Azmy

[Abstract of paper presented at the American Nuclear Society Topical Meeting on Advances in Mathematics, Computation and Reactor Physics, Pittsburgh, PA, April 28-May 1, 1991; Proc. Vol. 1, p. 1.1 (1991)]

The $S_{N}$ method is an approximate approach used to solve the linear Boltzmann transport theory equation. This method requires a significant amount of memory and computational time which are limiting factors for its utility in real-life applications. In the past decade, the significant advancements in computer hardware design (vector and parallel processing) have provided the possibility of development of new parallel/vector software which can operate significantly faster than the older serial/scalar ones.

In recent years, several studies have been performed on the vector/parallel processing of the $S_{N}$ transport theory rnethod. This paper primarily discusses a parallel algorithm for the $S_{N}$ method which is based on decomposition of the spatial domain. Wienke and coworkers have performed studies on the energy domain and have obtained significant speedups. Azmy considered angular parallelization of a one-group $x-y$ nodal $S_{N}$ algorithm and recently Rhoades and Flanery performed angular parallelization for a $x-y-z$ $S_{N}$ transport formulation. Haghighat and Mattis considered angular parallelization of a curvilinear (i.e. spherical) $S_{N}$ algorithm. For parallelization based on the spatial domain decomposition only one study has been reported by Yavuz and Larsen who demonstrated the possibility of spatial decomposition for a slab geometry on a serial machine. In the present work, we develop a spatial parallel formulation for spherical geometry and study its behavior under different physical conditions. Then, we describe our implementation of the algorithm on the Cornell National Supercomputer Facility (CNSF) IBM 3090/600J with six processors and measure speedups for different problem sizes.

Research sponsored by U.S. Department of Energy.

*Pennsylvania State University, University Park, PA. 
4.70

\section{A USER'S MANUAL FOR MASH 1.0 - A MONTE CARLO ADJOINT SHIELDING CODE}

\section{J. O. Johnson}

(Abstract of ORNL/TM-11778, March 1992)

The Monte Carlo Adjoint Shielding Code System, MASH, calculates neutron and gamma-ray environments and radiation protection factors for armored military vehicles, structures, trenches, and other shielding configurations by coupling a forward discrete ordinates air-over-ground transport calculation with an adjoint Monte Carlo treatment of the shielding geometry. Efficiency and optimum use of computer time are emphasized. The code system includes the GRTUNCL. and DORT codes for air-over-ground transport calculations, the MORSE code with the GIFT5 combinatorial geometry package for adjoint shielding calculations, and several peripheral codes that perform the required data preparations, transformations, and coupling functions. MASH is the successor to the Vehicle Code System (VCS) initially developed at Oak Ridge National Laboratory (ORNL).

The discrete ordinates calculation determines the fluence on a coupling surface surrounding the shielding geometry due to an external neutron/gamma-ray source. The Monte Carlo calculation determines the effectiveness of the fluence at that surface in causing a response in a detector within the shielding geometry, i.e., the "dose importance" of the coupling surface fluence. A coupling code folds the fluence together with the dose importance, giving the desired dose response. The coupling code can determine the dose response as a function of the shielding geometry orientation relative to the source, distance from the source, and energy response of the detector.

This user's manual includes a short description of each code, the input required to execute the code along with some helpful input data notes, and a representative sample problem (input data and selected output edits) for each code.

Research sponsored by Defense Nuclear Agency.

4.71

\section{DRC2: A CODE WITH SPECIALIZED APPLICATIONS FOR COUPLING LOCALIZED MONTE CARLO ADJOINT CALCULATIONS WITH FLUENCES FROM TWO-DIMENSIONAL R-Z DISCRETE ORDINATES AIR-OVER- GROUND CALCULATIONS}

\author{
C. O. Slater
}

(Abstract of ORNL/TM-11873, January 1992)

The DRC2 code, which couples MASH or MASHX adjoint leakages with DORT 2-D discrete ordinates forward directional fluences, is described. The forward fluences are allowed to vary both axially and radially over the coupling surface, as opposed to the strictly axial variation allowed by the predecessor DRC code. Input instructions are presented along with descriptions and results from several sample problems. Results from the sample problems are used to compare DRC2 with DRC, DRC2 with DORT, and DRC2 with itself for the case of $x-y$ dependence versus no $x-y$ dependence of the forward fluence. The test problems demonstrate that for small systems DRC and DRC2 give essentially the same results. Some significant differences are noted for larger systems. Additionally, DRC2 results with no $x-y$ dependence of the forward directional fluences are practically the same as those calculated by DRC.

Research sponsored by U.S. Department of Energy and by the Deferse Nuclear Agency. 


\section{COMPETITIVE SYSTEMS}

4.72

\section{DCOR: A DETERMINISTIC COMBAT MODEL CODE}

\author{
Y. Y. Azmy
}

(Abstract of ORNL/TM-11690, April 1991)

ORNL's deterministic combat model based on a system of PDEs is used to develop a flexible, user-friendly computer code called DCOR, for Deterministic Combat model of Oak Ridge, and previously known as WAR. The numerical solution of the PDEs is achieved via the Method of Lines (MOL) which approximates the spatial derivatives on a finite mesh yielding a set of ODEs that are solved using the Gear B Method. A general purpose software based on the MOL called PDETWO is used, with some modifications in DCOR, whereby the diffusion terms are approximately by a five-point finite-difference scheme and the convective terms are approximately by an upwind finite-difference representation. DCOR is an interactive code with graphical display capability for the solution, that permits external control by the user to simulate a wargame environment. A guide to the user of DCOR is included to help in setting up and modifying the input, either interactively or from a file. Preliminary results pertaining to the validation of the deterministic model with respect to a Monte Carlo simulation, to the accuracy of the numerical solution as the spatial mesh is refined, and to the versatility of DCOR is conducting sensitivity analyses are presented.

Research sponsored by U.S. Department of Energy.
4.73

\section{LIE GROUP ANALYSIS FOR A MULTISPECIES, SPATIALLY INHOMOGENEOUS, MUTUALLY INTERACTING GAS MIXTURE}

\author{
Y. Y. Azmy \\ V. C. Boff* \\ V. Protopopescu \\ J. Mandrekas ${ }^{\dagger}$
}

[Abstract of Transport Theory \& Statistical Physics 21. 119 (1992)]

We consider systems of general nonlinear PDEs that arise in the extended kinetic theory of gases describing mixtures of spatially inhomogeneous, mutually interacting species. Via Lie group analysis methods, we show that for general, autonomous, space-independent interaction laws, these systems are always invariant under the translation group. For a Lotka-Volterra interaction law and three or more species, we show that the system is invariant under a three-parameter group of transformations representing scaling, and translation in time and space.

Research sponsored by U.S. Department of Energy.

"University of Rome, "La Sapienza," via Eudossiana, Rome. Italy.

${ }^{\dagger}$ Georgia Institute of Fechnology, Atlanta, GA.

\subsection{4}

\section{SUSTAINABILITY AND PROFITABILITY IN ECOLOGICAL SYSTEMS WITH HARVESTING}

\section{S. J. Gaff $\quad$ V. Protopopescu}

(Abstract of ORNL/TM-12151, August 1992)

A simple model of economic and ecological interplay for a system of two interacting populations grown in a closed environment and harvested periodically for economic purposes was analyzed. The analysis was carried out by exploring the parameter space of the model, defined by a discrete map, a harvesting strategy, and an objective functional. Results showed nonmonotonicities of the outcome 
and sharp sensitivities that depend on the values of the parameters and that are caused by the discrete nature of the system. This approach may prove useful for solving problems that cannot be solved analytically and for providing some guidance in the management of complex systems.

Research sponsored by U.S. Department of Energy:

* Taylor University, Upland, IN

\subsection{5}

\section{GRAPHICS DEVELOPMENT OF DCOR: DETERMINISTIC COMBAT MODEL OF OAK RIDGE}

$$
\text { G. Hunt* Y. Y. Azmy }
$$

(Abstract of ORNL/TM-11973, October 1992)

DCOR is a user-friendly computer implementation of a deterministic combat model developed at ORNL. To make the interpretation of the results more intuitive, a conversion of the numerical solution to a graphic animation sequence of battle evolution is desirable. DCOR uses a coarse computational spatial mesh superimposed on the battlefield. This research is aimed at developing robust methods for computing the position of the combative units over the continuum (and also pixeled) battlefield, from DCOR's discretevariable solution representing the density of each force type evaluated at gridpoints. Three main problems have been identified and solutions have been devised and implemented in a new visualization module of DCOR. First, there is the problem of distributing the total number of objects, each representing a combative unit of each force type, among the gridpoints at each time level of the animation. This problem is solved by distributing, for each force type, the total number of combative units, one by one, to the gridpoint with the largest calculated number of units. Second, there is the problem of distributing the number of units assigned to each computational gridpoint over the battlefield area attributed to that point. This problem is solved by distributing the units within that area by taking into account the influence of surrounding gridpoints using linear interpolation. Finally, time interpolated solutions must be generated to produce a sufficient number of frames to create a smooth animation sequence. Currently, enough frames may be generated either by direct computation via the PDE solver or by using linear programming techniques to linearly interpolate intermediate frames between calculated frames.

Research sponsored by Defense Nuclear Agency and U.S. DOE.

* Georgia Institute of Technology, Atlanta, GA.

\subsection{6}

\section{TOPOLOGICAL CHAOS FOR A CLASS OF LINEAR MODELS}

\section{Protopopescu Y. Y. Azmy}

(Abstract of Mathematical Methods and Models in Applied Sciences 2, 79 (1992)]

We construct an example of linear rate equation in the Banach space of summable sequences, $l_{1}$, that exhibits the three properties required as signature of topological chaos, namely: (i) topological transitivity, (ii) dense periodic orbits, and (iii) positive Lyapounov exponents. The example is based on the properties of the backward shift operator on the Banach space $l_{1}$. Since linear chaos in the sense described above can occur only in an infinite dimensional setting, possible finitedimensional approximate manifestations are investigated. The relationship between the linear backward shift and the nonlinear Bernoulli shift is also discussed.

Research sponsored by U.S. Department of Energy.

4.77

\section{ANALYTIC MODELING VS SIMULATION FOR CLASSICAL WARFARE}

\author{
V. Protopopescu \\ R. T. Santoro \\ Y. Y. Azmy \\ B. L. Kirk
}

(Abstract of paper presented at the 22nd International Conference on Modeling and Simulation, Pittsburgh, PA, May 2-3, 1991; Proc. Vol. 5, p. 2480 (1991)]

Competitive situations-ranging from low intensity conflict to conventional combat-are modeled here by a system of partial differential equations (PDEs) with nonlinear interactions. The main features and advantages of the PDE model are discussed in connection with other classes of 
models, namely, ordinary differential equations (ODEs) analytic models and large-scale computer simulations.

An application to conventional warfare is reported which: (i) replicates successfully both attrition and maneuver, (ii) is in excellent agreement with the results of a simulation test-case, and (iii) lends itself to quick and inexpensive sensitivity analyses.

Future developments, possible spin-offs, and outstanding problems (mainly the validation problem) are briefly discussed.

Research sponsored by U.S. Department of Energy.
4.78

\section{COMBAT MODELING WITH IMPRECISE DATA}

\author{
V. Protopopescu R. Yager \\ J. Dockery ${ }^{\dagger}$
}

[Abstract of Int. J. of Intelligent Systems 7, 277 (1992)]

We apply the fuzzy description to some analytic models of combat, based on ordinary differential equations. The extension to a more involved partial differential equations model is briefly mentioned. The merits of the fuzzy description vs. the deterministic or probabilistic descriptions are discussed.

Research sponsored by U.S. Department of Energy.

* Iona College, New Rochelle, NY.

†Organization of the Joint Chiefs of Staff. The Pentagon, Washington, DC.

\section{SENSITIVITY AND UNCERTAINTY ANALYSIS}

4.79

\section{GRESS VERSION 2.0 USER'S MANUAL}

\section{J. E. Horwedel ${ }^{*}$}

(Abstract of ORNL/TM-11951, November 1991)

The primary objective of this manual is to provide a description of GRESS and to explain how to use GRESS to enhance FORTRAN 77 models for gradient calculation.

The use of the GRESS precompiler, SYMG, is presented. A complete description of how to enhance a source code for either forward or reverse propagation of derivatives using the chain rule is provided.

Programming information is also provided to aid in the installation and maintenance of the software.

Research sponsored by U.S. Department of Energy.

*Computing and Telecommunications Division.
4.80

\section{GRESSING ALONG}

\section{J. E. Horwedel*}

[Abstract of paper presented at The Institute of Management Science/Operations Research Society of America (TIMS/ORSA) Joint National Meeting, Nashville, TN, May 12-15, 1991; Proc. Vol. 31, p. 63 (1991)]

A method for performing a comprehensive sensitivity analysis using computer calculus is described. The procedure employs an automated system called GRESS used to enhance a FORTRAN computer code by adding derivative-taking capabilities. From a single run of an enhanced model, GRESS calculates and reports normalized sensitivities of selected results with respect to all input data.

Research sponsored by U.S. DOE National Low-Level Waste Management.

*Computing and Telecommunications Division. 


\subsection{1}

\section{AN AUTOMATED SENSITIVITY ANALYSIS PROCEDURE APPLIED TO A DISSOLVED OXYGEN SIMULATION MODEL}

\author{
J. E. Horwedel ${ }^{*}$ S. F. Railsback ${ }^{\dagger}$ \\ H. I. Jager ${ }^{\dagger}$
}

[Abstract of paper presented at the National Conference of American Socjety of Civil Engineers, Water Resource Planning Division on Water Resources Planning and Management, New Orleans, LA, May 20-22, 1991; Proc. Vol. 18, pp. 484488 (1991)]

Sensitivity and uncertainty analyses are necessary components in the evaluation of computer models utilized in support of performance assessment activities. The identification of important model parameters is valuable for ascertaining specific data needs and as a first step in a thorough assessment of parameter uncertainties. This report describes an automated procedure for performing a comprehensive sensitivity analysis making use of computer calculus. The procedure employs an automated system called GRESS that utilizes a precompiler to enhance a FORTRAN computer code by adding derivative-taking capabilities. From a single run of an enhanced model, GRESS calculates and reports normalized sensitivities of selected results with respect to all input data. The automated GRESS procedure is applied to the dissolved oxygen (DO) simulation model developed by the Oak Ridge National Laboratory (ORNL) to assess cumulative impacts of hydropower development at the navigation locks and dams in the upper Ohio River Basin. The sensitivity calculations for a sample problem show good agreement with those obtained by perturbation analysis. Results demonstrate than an automated procedure can be used cheaply and efficiently to perform a comprehensive sensitivity analysis of existing computer models. A methodology for developing enhanced versions of assessment models that include the capability to routinely report sensitivities for important parameters is discussed.

Research sponsored by U.S. DOE National Low-Level Waste Management.

\footnotetext{
* Computing and Telecommunications Division
}

$\dagger_{\text {Environmental Sciences Division. }}$
4.82

\section{AUTOMATED SENSITIVITY \\ ANALYSIS OF AN ATMOSPHERIC DISPERSION MODEL}

\author{
J. E. Horwedel ${ }^{*}$ R. J. Raridon* \\ R. Q. Wright*
}

[Abstract of Atmospheric Environment 26A(9), 1643 (1992)]

Sensitivity analyses is a necessary component in the evaluation of computer models utilized in support of performance assessment activities. Understanding the behavior of predictive models with respect to input data is important for (1) verifying the validity of a model, (2) determining parameters for which it is important to have accurate values, and (3) understanding the behavior of the system being modeled. This paper describes an automated procedure for performing a comprehensive sensitivity analysis using computer calculus. The procedure employs an automated system called GRESS that utilizes a precompiler to enhance a FORTRAN computer code by adding derivative-taking capabilities. From a single run of an enhanced model, GRESS calculates and reports normalized sensitivities of selected results with respect to all input data. GRESS computes a normalized sensitivity by multiplying a derivative by its associated input parameter value and dividing by the associated output value. The automated GRESS procedure is applied to the code AIRDOS-EPA, which estimates radiation doses caused by atmospheric dispersion of radionuclides around nuclear facilities. The sensitivity calculations for a sample problem show good agreement with those obtained by perturbation analysis. Results demonstrate that an automated procedure can be used cheaply and efficiently to perform a comprehensive sensitivity analysis of existing computer models. A methodology for developing enhanced versions of assessment models that include the capability to report routinely sensitivities for important parameters is discussed. Both GRESS and AIRDOS-EPA are available from the Radiation Shielding Information Center at Oak Ridge National Laboratory, P.O. Box 2008.

Research sponsored by U.S. Department of Energy.

*Computing and Telecommunications Division. 


\section{THEORETICAL ENGINEERING}

\subsection{3}

\section{ELECTRIC POWER GENERATION BY STEADY FLOW LIQUID METAL MHD GENERATORS}

\author{
C. M. Haaland
}

(Abstract of paper presented at the 1991 IEEE Interna tional Conference on Plasma Science, Williamsburg, VA, June 3-5, 1991; Proc. p. 200 (1991)]

Equations were derived which specify parameters for maximum efficiency [(electric power out)/(fluid power in)] MHD electric generation by steady flow of liquid metal (LM) in a Hartmann MDH geometry with Hartmann numbers $M>30$. For any selected efficiency, fluid velocity, magnetic induction, and power out, the duct volume is least for LMs having a maximum value of $(\sigma / \rho \nu)$, where $\sigma$ is the electrical conductivity, $\rho$ is the density, and $\nu$ is the kinematic viscosity. Minimum values were calculated for duct volume, mass, power out, pressure head, current, voltage and current density corresponding to efficiencies ranging from $84 \%$ to $99.9 \%$ for $\mathrm{Hg}, \mathrm{Na}$, and $\mathrm{NaK}$, for fluid velocities of $1,10,35$, and 100 $\mathrm{m} / \mathrm{s}$, and magnetic inductions of 1 and $4 \mathrm{~T}$. Duct volume included an entry length required for the LM to attain laminar flow. Efficiencies of $99 \%$ and higher can be attained only by using very large ducts and high power. For example, the minimum size duct at $99 \%$ efficiency using liquid $\mathrm{Na}$ at $371 \mathrm{~K}$ and magnetic induction of $4 \mathrm{~T}$, obtainable with superconducting electromagnets, has a gap spacing of $0.16 \mathrm{~m}, \mathrm{LM}$ duct mass of $370 \mathrm{~kg}$, and produces $370 \mathrm{MW}$ with fluid velocity of $35 \mathrm{~m} / \mathrm{s}$, pressure head of $42 \mathrm{MPa}$, and a power density of $920 \mathrm{MW} / \mathrm{m}^{3}$. In comparison, to obtain the same efficiency using $\mathrm{Hg}$ at $293 \mathrm{~K}$, at the same magnetic induction and fluid velocity, the minimum size duct has a gap spacing of $0.77 \mathrm{~m}, \mathrm{LM}$ duct mass of $614,000 \mathrm{~kg}$, power output of $4610 \mathrm{MW}$, pressure head of $23 \mathrm{MPa}$, and power density of $102 \mathrm{MW} / \mathrm{m}^{3}$. Minimum duct volume and corresponding minimum power output increase rapidly with small increases in efficiency above $99 \%$.

Research sponsored by U.S. Department of Energy.
4.84

\section{MINIMUM ENGINE SIZE \\ FOR OPTIMUM AUTOMOBILE ACCELERATION}

\author{
C. M. Haaland \\ [Abstract of Am. J. Phys. 60(5), 415 (1992)]
}

The theoretical minimum for engine power rating required to accelerate a vehicle of mass $M$ to velocity $V$ in time $T$ is shown to result from acceleration at constant maximum power. Because actual conditions do not allow infinite starting acceleration, the power per unit mass to provide optimum feasible acceleration with minimum engine power rating is given by $P_{o p t} / M=a_{\max }{ }^{2} T_{c}$, where $a_{\max }$ is the maximum starting acceleration and $T_{c}$ is the crossover time from maximum acceleration to the curve of acceleration at constant maximum power. Comparison with analysis of performance data for 18 automobiles reveals that 17 achieve near-optimum power usage.

Research sponsored by U.S. Department of Energy. 
Section 5

ENGINEERING PHYSICS AND MATHEMATICS

INFORMATION CENTERS 


\title{
5.0. INTRODUCTION
}

\author{
R. W. Roussin
}

Under the management umbrella of Engineering Physics Information Centers (EPIC), we operated three activities during the reporting period, April 1, 1991 December 31, 1992. The Radiation Shielding Information Center (RSIC), founded in FY 1963, serves the international scientific community as a technical institute and technology resource for radiation transport and safety. The second, Alplied Nuclear Data Development, focuses on providing processed data, usually multigroup cross sections, for use in radiation transport computer codes. The third ictivity involves participation in the shielding analysis part of the Safety Analysis Reports for Packaging for the Y-12 radioactive material container and shipment program.

\section{Radiation Shielding Information Center (RSIC)}

During this reporting period, RSIC observed the 30th year of operation at ORNL. Leading up to this milestone, several significant changes were made in the physical facilities. A major remodelling was accomplished through the installation of modern functional work units. In addition, the staff was supplied with personal computers linked by a local area network. Workstations based on RISC technology were acquired and are used not only for testing software packages but also for independent calculations. These changes were very timely because the RSIC user community is rapidly ming to $\mathrm{PC}$ and workstation environments and we were able to accommodate their char jing demands.

Established to promote the exchange of radiation transport technology, RSIC acts as a resource base for both government and civilian agencies in the United States and in foreign countries and pe: orms such diverse functions as testing, assembling, and distributing computer codes; testing and distributing multigroup cross-section libraries, both fine-group and broad-group; collecting and distributing other types of data bases; holding seminars to educate the community of particular techniques, especially computer-based techniques for solving radiation transport or nuclear data problems; helping to establish shielding benchmark problems and shielding standards; providing bibliographic information; and generally providing problem solving assistance to requesters. Staff members also engage in radiation transport developments and perform radiation transport studies, often in collaboration with staff members in EPMD as well as other institutions.

The RSIC sccpe includes the physics of interaction of radiation with matter; radiation production, jrotection, transport and safety; radiation detectors and measurements; re.co or physics and critica.:y safety; atmospheric dispersion, envirc.mental exposure, and waste management; shielding materials properties; computer codes useful in research and design; and nuclear data compilations. The goals of RSIC are to function as a technical institute to provide information (computer codes, technical advice, bibliographic and other data,) upon request; collect, evaluate, enrich, distill, and repackage information to extend the state of the art, bringing into the public domain technology more usable and more valuable than the sum of tr. e input; and to initiate and effect research and development in appropriate areas of need. 
RSIC has continued to participate in the work of the Cross Section Evaluation Working Group (CSEWG) to produce the U. S. Evaluated Nuclear Data File (ENDF), with the Methods and Formats Subcommittee chaired by the RSIC director. Other staff members are actively involved in CSEWG and also in the American Nuclear Society (ANS) in developing radiation protection and shielding standards and standards for scientific computer programming and its documentation.

In the area of the transfer of computing technology, RSIC now makes available over 900 computer code packages and 160 data packages. RSIC entered into an agreement with the Department of Energy's Energy Science and Technology Software Center (ESTSC) to be the sole dissemination source for DOE software developed in the technical area of radiation transport and safety. In addition, an RSIC staff member serves as liaison to collect and forward to ESTSC non-RSIC computing technology developed throughout Martin Marietta Energy Systems.

In terms of reciprocity in international information exchange, a significant percentage of all incoming computing technology (codes and data) comes from foreign contributors, with Japan and France continuing in the lead. Much of the new technology is of domestic interest.

RSIC interacts daily with, and continues to have the support of its user community, which is quite diverse and serves a broad range of government and privately sponsored programs. Because of the value of RSIC services, several new sponsors provided funding on behalf of particular segments of this user community.

\section{Applied Nuclear Data Development (ANDD)}

During this reporting period work has continued in the development of fineand broad-group cross-section data libraries. The 174 neutron, 38 photon group VITAMIN-E general-purpose cross-section library based on ENDF/B-V (released in 1979) now contains 94 materials. It has been used with success for fusion neutronics, fast reactor core and shielding applications, and various other radiation transport calculations within the division and externally.

ENDF/B-VI has recently been released and NADD has processed a selected set of materials into the VITAMIN-J 175n, 42g group structure (a superset of VITAMIN-E) in support of Defense Nuclear Agency work on Hiroshima/Nagasaki and other studies. Data for iron isotopes was also processed for LWR pressure vessel dosimetry studies and cited by the Nuclear Regulatory Commission for its improved performance over that previously available.

Based on this early experience, the production of a major processed cross-section library for Light Water Reactor (LWR) shielding is now under way. Processing of the ENDF/B-VI reference data into a 199n, $42 \mathrm{~g}$ data set is being done using the NJOY system developed at LANL. This fine-group data will be translated into AMPX format, undergo a data testing program, and ultimately be used to develop a successor to the BUGLE broad group cross-section library, based on ENDF/B-IV, which has served as the standard for LWR shielding since 1980.

In order to foster the use of newly evaluated data, NADD coordinates an international multigroup intercomparison activity which was initiated as Subgroup 7 of the Nuclear Energy Agency Nuclear Science Committee Working Group 
on International Evaluation Cooperation. Processed data libraries based on the VITAMIN-J group structure are being generated from evaluated data libraries from the United States, Europe, and Japan. To facilitate intercomparisons, the ANDD has sponsored the development of a code that translates the multigroup data into the AMPX format that is commonly used at ORNL and other sites around the world.

\section{Safety Analysis Reports for Packaging}

EPIC staff members are in charge of performing the radiation shielding analysis and preparing the shielding chapter for all the Safety Analysis Reports for Packaging (SARPS) for the Y-12 Plant radioactive material container and shipment program. Besides the initial draft, three separate chapter versions are prepared: issued for comment (IFC), issued for approval (IFA), and issued approved (IA). Each version must be approved by three review committees for compliance with the Code of Federal Register (CFR) Title 10 Part 71, dealing with the packaging and transportation of radioactive material. These committees are an independent MMES group, (2) an Albuquerque-based group reporting to DOE Albuquerque Operations, and (3) a group from LLNL reporting to Headquarters. Upon approval of the SARP from all committees, a transportation certificate for the package is issued by DOE. 


\section{RADIATION TRANSPORT DEVELOPMENTS}

5.1

\section{IMPLEMENTATION OF THE MONTE CARLO CHARGED PARTICLE TRANSPORT CODE EGS4 ON THE HYPERCUBE}
B. L. Kirk
Y. Y. Azmy
T. A. Gabriel
C. Y. Fu

(Abstract of paper presented at the American Nuclear Society Topical Meeting on New Horizons in Radiation Protection and Shielding, Pasco, WA, April 26-30, 1992; Proc. pp. 34-37 (1992)]

Monte Carlo transport codes are commonly used in the study of particle interactions. The CALOR89 code system is a combination of several Monte Carlo transport and analysis programs. In order to produce good results, a typical Monte Carlo run will have to produce many particle histories. On a single processor computer, the transport calculation can take a huge amount of time. However, if the transport of particles were divided among several processors in a multiprocessor machine, the time can be drastically reduced.

Research sponsored by U.S. DOE Office of Superconducting Supercomputing Colliders.
5.2

\section{ANALYTIC ESTIMATION OF RADIATION EFFECTS IN SPACE USING SOLID ANGLES}

\author{
S. N. Cramer
}

[Abstract of paper presented at the American Nuclear Society Topical Meeting on Nuclear Technologies for Space Exploration, Jackoson Hole, WY, August 16-19, 1992; Proc. Vol. III, pp. 654-663 (1992)]

The void of space allows the possible simplification of certain integrals which describe the transport of radiation from a point to a surface or volume. The solid angle in this integral is investigated analytically for geometric figures associated with the conic sections, their volumes of revolution, and other plane surfaces. The selection of random directions within the solid angles and the difficulties associated with concave surfaces are discussed. The expression for each solid angle is manipulated into an integral form which can be found in an appropriate handbook reference. It is found that the solid angles are given as linear combinations of elliptic integrals and elementary functions.

Research sponsored by U.S. DOE Office of Nuclear Energy Research and Development.

\section{APPLIED NUCLEAR DATA DEVELOPMENTS}

5.3

\section{NEW GAMMA-RAY BUILDUP FACTOR DATA FOR POINT KERNEL CALCULATIONS: ANS-6.4.3 STANDARD REFERENCE DATA}

\section{K. Trubey}

(Abstract of ORNL/RSIC-49/R1, August 1991)

An American Nuclear Society Standards Committee Working Group, identified as ANS6.4.3, has developed a set of evaluated gamma-ray isotropic point-source buildup factors and attenuation coefficients for a standard reference data base. The largely unpublished set of buildup fac- tors calculated with the moments method has been evaluated by recalculating key values with Monte Carlo, integral transport, and discrete ordinates methods. Additional buildup factor data were obtained from PALLAS code results. Attention has been given to frequently-neglected processes such as bremsstrahlung and the effect of introducing a tissue phantom behind the shield. The proposed draft standard, provided as an appendix, contains data for a source energy range from $15 \mathrm{keV}$ to 15 $\mathrm{MeV}$ and for 22 elements and 3 mixtures (water, air, and concrete). The buildup factor data are also represented as coefficients for the G-P fitting function. Tables giving correction factors for multiple scattering in tissue are also provided.

Research sponsored by Office of Nuclear Regulatory Research. 
5.4

\section{SPECIFICATIONS FOR THE DEVELOPMENT OF BUGLE-93: AN ENDF/B-VI MULTIGROUP CROSS SECTION LIBRARY FOR LWR SHIELDING AND PRESSURE VESSEL DOSIMETRY}

\author{
J. E. White \\ R. Q. Wright* \\ R. W. Roussin \\ D. T. Ingersoll
}

(Abstract of ORNL/TM-12230, November 1992)

Specifications have been developed for a new multigroup cross section library based on ENDF/B-VI data for light water reactor shielding and reactor pressure vessel dosimetry applications. The resulting broad-group library and an intermediate fine-group library are defined by the specifications provided in this report. Processing ENDF/B-VI into multigroup format for use in radiation transport codes will provide radiation shielding analysts with the most currently available nuclear data. It is expected that the general nature of the specifications will be useful in other applications such as reactor physics.

Research sponsored by U.S. Nuclear Regulatory Cormmission and U.S. DOE.

*Computing and Telecommunications Division.

\section{5}

\section{INTERNATIONAL EVALUATION COOPERATION SUBGROUP 7: MULTIGROUP CROSS SECTION PROCESSING}
R. W. Roussin E. Sartori*
J. E. White
R. MacFarlane $e^{\ddagger}$
G. Panini ${ }^{\dagger}$
M. Mattes\#
D. Muir $\S$
H. Takano**
I. Hasegawa**
F. Mann ${ }^{* * *}$

(Abstract of paper presented at the International Conference on Nuclear Data for Science and Technology, Julich, Federal Republic of Germany, May 13-17, 1991; Proc. pp. 860-862, S. M. Qaim, Ed., Springer-Verlag (1992)]

The chairmen of the ENDF/B, JEF, EFF, and JENDL evaluated data files adopted a proposal to develop a fine-group processed cross section library based on the "VITAMIN" concept. The authors listed above, with support from oth- ers, are participating in this project. The end result will be a pseudo-problem-independent finegroup cross section library generated from the latest evaluated data in ENDF/B-VI, JEF-2, EFF2, and JENDL-3. Initial applications of the library will be for shielding, fast reactor physics, and fusion neutronics. Progress made to date will be discussed. (Cross sections, EFF, ENDF, JEF, JENDL, multigroup, radiation transport, shielding).

Research sponsored by U.S. Department of Energy.

*Nuclear Energy Agency Data Bank, Saclay, France.

†ENEA, Bologna, Italy.

${ }^{\ddagger}$ Los Alamos National Laboratory, Los Alamos, NM.

$\oint_{\text {IAE }}$

$\oint_{\text {IAEA, Nuclear Data Section, Vienna, Austria. }}$

\# IKE, Stuttgart Technical University, Germany.

** Japan Atomic Energy Research Institute, Tokaj-mura, Japan.

*** Hanford Engineering Development Laboratory, Richland, WA.

5.6

\section{TRANSPORT CALCULATIONS OF NEUTRON TRANSMISSION THROUGH STEEL USING ENDF/B-V, REVISED ENDF/B-V AND ENDF/B-VI IRON EVALUATIONS}
M. L. Williams* M. Asgari*
C. Aboughantous*
R. Q. Wright ${ }^{\dagger}$
J. E. White
F. B. K. $\operatorname{Kam}^{\dagger}$

[Abstract of Ann. Nucl. Energy 18(10), 549 (1991)]

The ENDF/B-VI evaluated nuclear data file has been recently released by the U.S. National Nuclear Data Center during 1990. Among the most eagerly awaited new cross-section evaluations in this data collection are those for the natural iron isotopes, due to their importance in nuclear systems analysis and because the previous ENDF/B data (version V, which was released in 1979) are known to underestimate the transmission of fast neutrons through steel structures such as reactor pressure vessels and radiation shielding. In this paper, a comparison is made of results obtained from neutron transport calculations performed with these two ENDF/B version (V 
and VI) of iron data as well as an intermediate, revised version $\mathrm{V}$ evaluation that was proposed in 1986. Several different response parameters that are sensitive to high-energy neutrons are examined, for a variety of geometrical configurations and source spectra. It is found that the two newer iron evaluations substantially increase the transmission of high-energy neutrons through steel components with an incident fission spectrum source. Preliminary estimates indicate that the version VI iron evaluation will considerably improve the agreement between calculations and experimental dosimeter measurements used in light water reactor pressure vessel fluence analysis. The calculated leakage spectrum of D-T fusion neutrons from an iron sphere is also improved for energies above $4 \mathrm{MeV}$, but large discrepancies with the measured spectrum are still observed at lower energies.

Research sponsored by U.S. DOE and U.S. Defense Nuclear Agency,

*Louisiana State University, Baton Rouge, LA.

$\dagger$ Computing and Telecornmunications Division.

\section{RADIATION TRANSPORT STUDIES}

\section{7}

\section{SHIELDING DESIGN METHODS OF A SYNCHROTRON RADIATION FACILITY PART I: ACCELERATOR}

\section{S. Ban* H. Hirayama*}

(Abstract of ORNL/TR-91/4, April 1991)

Various problems for designing the shield of a synchrotron radiation facility are summarized and discussed. The characteristics of the radiation fields and of the administration of radiation protection around the facility are also presented.

Research sponsored by U.S. Department of Energy.

* National Laboratory for High Energy Physics, Ibarakjken, Japan.

\section{8}

\section{TIME-DEPENDENT MONTE CARLO CALCULATIONS OF THE OAK RIDGE ELECTRON LINEAR ACCELERATOR TARGET NEUTRON SPECTRUM}

\section{S. N. Cramer F. G. Perey}

Abstract of Nucl. Sci. Eng. 111, 102 (1992)]

The time-dependent spectrum of neutrons in the water-moderated Oak Ridge Electron Linear Accelerator (ORELA) target is calculated using a modified version of the MORSE multigroup Monte
Carlo code with an analytic hydrogen scattering model. Distributions of effective neutron distance traversed in the target are estimated with a timeand energy-dependent algorithm from the leakage normal to the target face. The $10-\mathrm{eV}$ to 20 $\mathrm{MeV}$ energy range is adequately represented in the MORSE code by the 174-group VITAMIN-E cross-section library with a $P_{5}$ expansion. An approximate representation of the ORELA positron source facility, recently installed near the target, is included in the calculations to determine any perturbations the positron source might create in the computed neutron distributions from the target. A series of coupled Monte Carlo calculations is performed from the target to the positron source and back to the target using a next-event estimation surface source for each step. The principal effect of the positron source is an increase in the distance for the lower energy neutron spectra, producing no real change in the distributions where the ORELA source is utilized for experiments. Different configurations for the target are investigated to simulate the placement of a shadow bar in the neutron beam. These beam configurations include neutrons escaping from (a) the central tantalum plates only, (b) the entire target with the tantalum plates blocked out, and (c) only a small area from the water. Comparisons of the current data with previous calculations having a less detailed model of the tantalum plates are satisfactory.

Research sponsored by U.S. DOE Office of Nuclear Physics. 
5.9

\section{INVESTIGATION OF RADIATION EFFECTS IN HIROSHIMA AND \\ NAGASAKI USING A GENERAL MONTE CARLO-DISCRETE ORDINATES COUPLING SCHEME}

\section{S. N. Cramer \\ C. O. Slater}

[Abstract of $\mathrm{Nucl}$. Sci. Eng. (in press)]

A general Monte Carlo-discrete ordinates radiation transport coupling procedure has been cre- ated to study effects of the radiation environment in Hiroshima and Nagasaki due to the bombing of these two cities. The forward two-dimensional free-field air-over-ground flux is coupled with an adjoint Monte Carlo calculation. The size, orientation, or translation of the Monte Carlo geometry is unrestricted. The radiation effects calculated are the dose in the interior of a large concrete building in Nagasaki and the activation production of Co-60 and P-32 in Hiroshima.

Research sponsored by U.S. DOE and U.S. Defense Nuclear Agency.

\section{INFORMATION ANALYSIS CENTER ACTIVITIES}

5.10

\section{JANE: A NEW INFORMATION RETRIEVAL SYSTEM FOR THE RADIATION SHIELDING INFORMATION CENTER}

\author{
D. K. Trubey
}

(Abstract of ORNL/RSIC-53, May 1991)

A new information storage and retrieval system has been develnped for the Radiation Shielding Information Center (RSIC) at Oak Ridge National Laboratory to replace mainframe systems that have become obsolete. The database contains citations and abstracts of literature which were selected by RSIC analysts and indexed with terms from a controlled vocabulary. The database, begun in 1963, has been maintained continuously since that time.

Comparisons of the new and presently-used systems for actual searches of the literature indicate that it is practical to replace the mainframe systems with a minicomputer system similar to the present version of JANE.

Research sponsored by U.S. Department of Energy.

\subsection{1}

\section{DETERMINISTIC METHODS IN RADIATION TRANSPORT}
A. F. Rice
R. W. Roussin

(Abstract of ORNL/RSIC-54, June 1992)

The Seminar on Deterministic Methods in Radiation Transport was held February 4-5, 1992, in Oak Ridge, Tennessee. Eleven presentations were made and the full papers are published in this report, along with three that were submitted but not given orally. These papers represent a good overview of the state of the art in the deterministic solution of radiation transport problems for a variety of applications of current interest to the RSIC user community.

Research sponsored by U.S. Department of Energy. 
5.12

COMPUTER CODES FOR CHECKING, PLOTTING AND PROCESSING OF NEUTRON CROSS-SECTION COVARIANCE DATA AND THEIR APPLICATION

\author{
E. Sartori* \\ R. W. Roussin
}

[Abstract of paper presented at the Specialists Meeting on Evaluation and Processing of Covariance Data, Oak Ridge National Laboratory, Oak Ridge, TN, October 7-9, 1992; Proc. pp. 253-264 (1993)]

This paper presents a brief review of computer codes concerned with checking, plotting, processing and using of covariances of neutron cross-section data. It concentrates on those available from the computer code information centers of the United States and the OECD/Nuclear Energy Agency.

Emphasis will be placed also on codes using covariances for specific applications such as uncertainty analysis, data adjustment and data consistency analysis.

Recent evaluations contain neutron cross section covariance information for all isotopes of major importance for technological applications of nuclear energy. It is therefore important that the available software tools needed for taking advantage of this information are widely known as they permit the determination of better safety margins and allow the optimization of more economical designs of nuclear energy systems.

Research sponsored by U.S. Department of Energy.

* OECD/NEA Data Bank, France. 
APPENDICES 


\section{PERSONNEL ACTIVITY}

\section{NEW STAFE MEMBERS}

\section{A. Scientific Staff}

K. R. Bennett, Householder Fellow, Ph.D., Mathematics, University of Kentucky.

J. C. Gehin, Ph.D., Nuclear Engineering, Massachusetts Institute of Technology.

B. D. Semeraro, Ph.D., Computer Science, University of Ilinois.

W. A. Shelton, Ph.D., Physics, University of Cincinnati.

E. C. Uberbacher, Ph.D., Chemistry/Biophysics, University of Pennsylvania.

\section{B . Administrative and Technical Support Staff}

K. W. Bateman, Computer Engineering, Co-Op Student

P. S. Brantley, Nuclear Engineering, Part-time Technical Support

S. W. Case, Communications, Summer Clerical

M. D. Dewing, Computer Science, Summer Technical Support

M. E. Hall, Secretary

T. R. Henson, Organizational Management, Secretary

C. A. Leete, Computer Science, Summer Technical Support

R. A. Lexvold, Electrical Engineering, Co-Op Student

B. B. Lowekamp, Chemistry/Computer Science, Co-Op Student

A. M. McCoy, Secretary

J. E. Nunn, Summer Clerical

C. C. Parks, Physics, Summer Technical Support

C. A. San Soucie, Mathematical Sciences, Summer Technical Support

D. J. Sept, Computer Science, Summer Technical Support

\section{STAFF TRANSFERS OUT AND TERMINATIONS}

A. Scientific Staff

G. de Saussure (deceased)

G. E. Liepins (deceased)

R. W. Peelle (retired)

C. M. Perey (retired)

\section{B . Administrative and Technical Support Staff}

K. W. Bateman (returned to school)

S. W. Casey (returned to school)

S. R. Damewood (Energy Division)

M. D. Dewing (returned to school)

M. A. Hacker (Metals and Ceramics Division)

C. A. Leete (returned to school)

R. A. Lexvold (returned to school)

B. B. Lowekamp (returned to school) 
J. E. Nunn (returned to school)

C. C. Parks (returned to school)

C. A. San Soucie (returned to school)

D. J. Sept (returned to school)

D. H. Wood (Exxon)

\section{DISTINGUISHED SCIENTIST}

J. J. Dongarra, University of Tennessee

\section{POST-DOCTORAL ASSIGNMENTS}

O. H. Doerum, Robotics Vision, Intelligent Systems Section

V. L. Eijkhout, Maihematics, Univ. of Tennessee

X. Guan, Computer Science/Human Genome, Intelligent Systems

R. Pozo, Computer Science, Univ. of Tennessee, Mathematical Sciences

K. Rahmani, Robotics/Mechanical Engineering, Intelligent Systems

J. Yoon, Robotics, Korea Atomic Energy Research Institute, Intelligent Systems

\section{GUESTS AND SHORT-TERM APPOINTMENTS}

\section{A. University/Industry Research Collaborators}

R. W. Allen, Systems Tech, Inc., Human Factors, Hermosa Beach, California

M. A. Abidi, Electrical/Computer Engineering, Univ. of Tennessee

Automated Sciences, Nuclear Engineering, Oak Ridge, Tennessee

J. L. Barlow, Computer Science, Pennsylvania State University

A. L. Beguelin, Computer Science, Univ. of Tennessee

J. R. S. Blair, Computer Science, Univ. of Tennessee

J. Borenstein, Robotics, Univ. of Michigan

R. W. Brockett, EPM Advisory Committee, Electrical Engineering/Computer

Science, Harvard University

R. W. Brown, Physics, Luther College

D. S. Carruth, Computer Science, Alabama Supercomputer Center

O. W. Christian, Physics (retiree)

T. C. Collins, High-Energy Physics, Univ. of Tennessee

J. B. Conway, Mathematics, Univ. of Tennessee

J. J. Dongarra, Computer Science, Univ. of Tennessee

D. J. Dudziak, EPM Advisory Committee, Nuclear Engineering, North Carolina State University

W. L. Dunn, Nuclear Engineering, Quantum Research Services, Inc.

C. M. Frankle, Nuclear Physics, Los Alamos National Laboratory

T. B. Fowler, Nuclear Engineering (retiree)

R. E. Funderlic, Mathematics, North Carolina State University

F. W. Garber, Nuclear Physics, Intraspec, Inc., Oak Ridge, Tennessee

G. Gatoff, Physics, Univ. of Tennessee

J. A. George, Computational Math, Univ. of Waterloo

R. Gonzalez, Robotics, Knoxville, Tennessee

A. T. Goshaw, High-Energy Physics, Duke University

$X$. Guan, Human Genome, Univ. of Tennessee

F. Guess, Statistics, Sabbatical, Univ. of Tennessee

R. Gwin, Nuclear Physics (retiree) 
B. L. Gyorffy, Physics, Univ. of Bristol, United Kingdom

T. Handler, High-Energy Physics, Univ. of Tennessee

H. J. Hargis, High-Energy Physics, Univ. of Tennessee

E. L. Hart, High-Energy Physics, Univ. of Tennessee

G. R. Hartman, Computer Science, Intel Corporation

N. W. Hill, Nuclear Physics(retiree)

P. Huffman, Nuclear Physics, Duke University

T. E. Hutchinson, Human Factors, Univ. of Virginia

M. E. Kitrell, Business Administration, Oak Ridge Associated Universities

D. S. Koltick, High Energy Physics, Purdue University

J. E. Leiss, EPM Advisory Committee, Nuclear Physics, DOE (retired)

J. W. H. Liu, Computer Science, York University

Y. Koren, Robotics, Univ. of Michigan

K. Kurisaka, Nuclear Fuel Development Corp., Japan

R. E. MacFarlane, Nuclear Engineering, Los Alamos National Laboratory

R. L. Macklin, Nuclear Physics (retiree)

D. R. Mackay, Civil Engineering, Intel Corporation

Roger L. Macklin, Nuclear Physics

F. C. Maienschein, Physics (retiree)

L. Mansfield, Mathematics, Univ. of Virginia

L. T. Machburn, High-Energy Physics, Univ. of Tennessee

B. F. Maskewitz, Mathematics (retiree)

E. A. Fleishman, Human Factors, George Mason University, Fairfax, Virginia

G. R. Montry, Physics, Univ. of Michigan

N. Moray, EPM Advisory Committee, Mechanical/Industrial Engineering/Psychology, Univ. of Illinois

M. C. Moxon, Nuclear Physics, Harwell, England

Y. I. Noy, Data Acquisition, Transport Canada, Ottawa, Ontario

J. M. Ortega, Mathematics, Univ. of Virginia

L. S. Ostrouchov, Computer Science, Univ. of Tennessee

J. K. Panakkal, High-Energy Physics, Argonne National Laboratory

R. W. Peelle, Nuclear Physics (retiree)

S. Petrov, Human Genome, Univ. of Tennessee

Y. P. Popov, Nuclear Physics, Joint Institute for Nuclear Physics, Russia

V. Prasanna, Computer Science, Univ. of Southern California

N. S. V. Rao, Robotics, Old Dominion University

F. D. Redman, Computer Science, Intel Corporation

J. J. Reidy, High-Energy Physics, Univ. of Mississippi

J. P..Riehs, Nuclear Physics, Institute fur Kernphysik, Austria

T. H. Rockwell, Human Factors, R\&R Research, Inc., Columbus, Ohio

Y. Roth-Tabak, Computer Science, Robotics, Univ. of Michigan

H. J. Schmiedmayer, Nuclear Physics, Institute fur Kernphysik, Austria

R. A. Schrack, Nuclear Physics, National Institute of Standards and Technology

M. B. Shah, Human Genome, Univ. of Tennessee

E. I. Sharapov, Nuclear Physics, Joint Institute for Nuclear Physics, Russia

L. R. Shenton, Statistics, Univ. of Georgia

A. Shono, Power Reactor and Nuclear Fuel Development Corporation, Japan

A. D. Solomon, Mathematics, Private Consultant

M. Stafford, Mathematics, U.S. Air Force Academy

R. C. Stirling, Engineering Administration, Kendall Square Research

R. Stohler, Kaman Sciences Corp., Albuquerque, New Mexico

K. Storjohann, Computer Vision/Robotics, Univ. of Mainz, Germany 
D. Straight, Computer Science, Univ. of Tennessee

M. Trivedi, Elec/Comp. Engineering, Univ. of Tennessee

D. K. Trubey, Computer Science (retiree)

L. S. Tonsunoglu, Robotics, Univ. of Texas

E. C. Uberbacher, Human Genome, Univ. of Tennessee

V. R. Uppuluri, Mathematics (retiree)

E. L. Wachspress, Mathematics, Univ. of Tennessee

J. Walters, Nuclear Physics, IntraSpec, Inc., Oak Ridge

D. Waltman, Computer Science, Univ. Corp. for Atmospheric Research, Bolder, Colorado

O. A. Wasson, Nuclear Physics, National Institute of Standards and Technology

David K. Wehe, Robotics, Dept. of NE, Univ. of Michigan

T. E. Weymouth, Robotics, Univ. of Michigan

M. F. Wheeler, EPM Advisory Committee, Computer Science, Rice University

J. R. White, Nuclear Engineering, Univ. of Lowell, Lowell, Massachusetts

D. W. Zachmann, Computer Science, Colorado State University

J. Ziebarth, Computer Science, Univ. of Alabama

\section{B . Eaculty Research Participation Program}

R. F. Carlton, Nuclear Physics, Middle Tennessee State University

J. C. Courtney, Nuclear Engineering, Louisiana State University

G. J. Davis, Mathematics, Georgia State University

G. Einstein, Engineering, ORNL/Tennessee State University Research Initiative

J. R. Goldberg, Psychology/Engineering, Sabbatical, Pennsylvania State University,

J. M. Kallfelz, Nuclear Engineering, Georgia Institute of Technology

H. N. Narang, Applied Mathematics, Tuskegee University

S. R. Seidel, Computer Science, Michigan Technological University

K. N. Sigmon, Mathematics, Univ. of Florida

C. A. Sparrow, Nuclear Engineering, Mississippi State University

R. R. Winters, Nuclear Physics, Denison University

M. S. Zien-Sabattou, Electrical Engineering, Tennessee State University Research Initiative

\section{Graduate Students Research Participation_Program}

M. B. Abdulghafour, Computer Vision, Univ. of Tennessee

R. F. Barrett, Mathematics, Univ. of Tennessee

S. Chaudhry, Nuclear Engineering, Univ. of Florida

P. V. Cherkukuri, Nuclear Engineering, Univ. of Florida

S. C. Chiang, Mechanical Engineering/Robotics, Univ. of Florida

H. Dai, Nuclear Engineering, Univ. of Florida

A. K. Dalmia, Robotics/Vision, Univ. of Tennessee

M. S. Elsasser, Automation Technology/Robotics, Fachhochschule fur

Technik and Wirtschaft Reutlingen, Stuttgart, Germany

W. Fabritz, Physics, Atominstitute, Austria

L. Feng, Mechanical Engr/Robotics, Univ of Michigan

J. M. Graham, Statistics, North Carolina State University

C. P. J. Halloy, Physics, Univ. of Tennessee

A. Hampapur, Computer Science, Univ. of Michigan

A. W. Hoover, Robotics, Univ. of South Florida, Tampa 
D. Ioannou, Engineering, Univ. of Florida

S. B. Jacobsen, Electrical Engineering/Robotics, Aalborg University, Aalborg, Denmark

M. S. Jensen, Electrical Engineering/Robotics, Aalborg University, Aalborg, Denmark

Y. Kim, Computer Science, Univ. of Tennessee

G. M. Kofler, Physics, Atominstitute, Austria

J. A. Kolodziejczyk, Mathematics, Univ. of Tennessee

S. Kopecky, Physics, Institut fur Kernphysik, Germany

S. Kristensen, Electrical Engineering, Robotics, Aalborg University, Aalborg, Denmark

J. Kruse, Nuclear Physics, Monmouth College, Illinois

L. L. Mann, Mathematics, Univ. of Tennessee

S. B. Marapane, Robotics/Computer Science, Univ. of Tennessee

J. D. Martens, Electrical Engineering/Robotics, Case Western Reserve

K. A. Morgansen, Mechanical Engineering/Robotics, Boston University

H. M. Nielsen, Electrical Engineering/Robotics, Aalborg University, Aalborg, Denmark

G. Rajeev, Computer Science, Univ. of Tennessee

C. Rakich, Nuclear Physics, Albion College, Michigan

$R$. Shreedhar, Mechanical Engineeririg/Robotics, Univ. of Texas

R. Siddarthan, Computer Science, Univ. of Tennessee

E. Sorensen, Electrical Engineering/Robotics, Aalborg University, Aalborg, Denmark

T. Steinel, Robotics, Fachhochschule Wiesbaden, Germany

N. A. Van Goor, Chem. Engineering, Delft University, Netherlands

Y. Watanabe, Robotics, University of Tsukuba, Japan

\section{Undergraduate_Student_Research Participation_Program}

J. R. Andoleo, Electrical Engineering/Robotics, Univ. of Miaami M. L. Ailor, Mathematics/Computer Science, Univ. of Tennessee

A. Bhatnagar, Computer Science/Physics, Coe College, Cedar Rapids, Iowa

W. Chen, Computer Science/Physics, Denison University

L. A. Fay, Computer Science/Mathematics, Hiram College, Cincinnati

D. M. Flory, Engineering Physics, Univ. of Michigan

C. R. Hartnell, Mathematics/Computer Science, Univ. of Tennessee

J. P. Iriarte, Mathematical Science, Barry University, Miami

E. S. Kirsch, Computer Science, Univ. of Tennessee

E. M. Neis, Engineering Physics, Univ. of Tennessee

J. A. Nichols, Mathematics, Univ. of Tennessee

K. J. McCollam, Nuclear Physics, Univ. of Minnesota

B. W. McCord, Computer Science, Alma College,

R. A. McNeese, Physics/Mathematics, Univ. of North Carolina

M. M. Moulton, Mathematics, Ilinois Institute of Technology

S. A. Moulton, Computer Science, Vanderbilt University

R. R. Murphy, Computer Science, Georgia Institute of Technology

E. M. Neeis, Engineering Physics, Univ. of Iowa

M. Ng, Electrical Engineering, Cornell University

D. M. Patrone, Computer Science, St. Bonaventure University

D. N. Pearson, Electrical Engineering/Engineering Physics, Univ. of North Dakota

L. A. Smith, Nuclear Engineering, Univ. of Missouri - Rolla

A. A. Szpiro, Mathematics, Univ. of California at San Diego

D. M. Thomas, Computer Science, Computer Science, Itta Bena MS Valley State 
F. A. Tulloch, Mathematics, Barry University, Miami

S. Wallace, Molecular Genetics, Univ. of Wien, Vienna, Austria

S. Y. Whitaker, Mathematics, Clark Atlanta University

M. Yeh, Cognitive Science/Applied Mathematics, Rice University

C. Zhao, Mathematics, Denison College, Columbus, Ohio 


\section{SCIENTIFIC AND PROFESSIONAL ACTIVITIES}

April 1, 1991 - December 31, 1992

V. Alexiades

Professor

- Mathematics Department, University of Tennessee, Knoxville, TN.

Award

- UTK-ORNL Science Alliance Research Incentive Award, 19861991.

Reviewer

- Mathematics Reviews

\section{Referee}

- Applicable Analysis

- SIAM Journal of Mathematical Analysis

- Nonlinear Analysis

- International Journal of Heat and Mass Transfer

- ASME Journal of Heat Transfer

- Rocky Mountain Mathematics Journal

- NSF and DOE Proposals

\section{F. S. Alsmiller}

\section{Co-chairman}

- Working Group on Simulation at International Workshop on Beam Induced Energy Deposition at International Workshop on Beam Induced Energy Deposition, SSC Laboratory, Dallas, TX, November 19-22, 1991.

Referee

- Nuclear Instruments and Methods in Physics Research

R. G. Alsmiller, Jr.

Co-chairman

- Working Group on Simulation at International Workshop on Beam Induced Energy Deposition at International Workshop on Beam Induced Energy Deposition, SSC Laboratory, Dallas, TX, November 19-22, 1991. 


\section{Member}

- Subcommittee on Dosimetry of the National Research Council Advisory Committee on the Radiation Effects Research Foundation.

- DOE Advisory Panel on Accelerator Radiation Safety.

\section{Referee}

- Nuclear Science and Engineering

- Nuclear Instruments and Methods in Physical Research

- Physics in Medicine and Biology

Y. Y. Azmy

Executive committee member

- Mathematics and Computation Division of the American Nuclear Society.

\section{Referee}

- Nuclear Science and Engineering

- Journal Computational Physics

- Transport Theory and Statistical Physics

\section{Invited papers}

- "General Order Nodal Transport Methods and Application to Parallel Computing," US-USSR Workshop on Numerical Transport Theory, College Station, TX, November 1991.

- "General Order Characteristic Methods for Solving Neutron Transport Problems," First International Symposium on Numerical Transport Theory, Moscow, Russia, May 1992.

- "Arbitrarily High Order Characteristic Methods for Solving the Neutron Transport Equation," Annals of Nuclear Energy 19, 593 (1992); special issue celebrating Prof. Devooght's sixtieth birthday.

\section{A. L. Bangs}

\section{Awards}

- MMES President's Award for Continuous Improvement

- NSF Summer Institute in Japan

C. O. Beasley

\section{Member}

- American Physical Society. 
Referee

- Nuclear Fusion

- Physics of Fluids

- Plasma Physics

Safety officer

- Engineering Physics and Mathematics Division.

Radiation control officer

- Engineering Physics and Mathematics Division.

Quality assurance coordinator

- Engineering Physics and Mathematics Division.

Training coordinator

- Engineering Physics and Mathematics Division.

Environmental protection officer

- Engineering Physics and Mathematics Division.

Facility manager

- Engineering Physics and Mathematics Division.

HAZCOM coordinator

- Engineering Physics and Mathematics Division.

\section{J. J. Beauchamp}

Referee

- Canadian Journal of Fisheries and Aquatic Sciences

- Communications in Statistics - Theory and Methods

- Environmental Toxicology and Chemistry

- Ecotoxicology

Invited lecture

- "What is Statistics?" 1991 DOE High School Science Honors Program, ORNL, July 23, 1991.

K. O. Bowman

Member

- The President's Committee on Employment of People with Disabilities.

- Committee on Statistics and Disability, American Statistical Association. 
- Advisory Board, Journal of Statistical Computation and Simulations.

- International Editorial Board, Communications in Statistics.

- International Organizing Committee, Indo-U.S.-Japan Conference.

- Advisory Board, Quality through Engineering Design, Indo-U.S. Japan Conference.

- Grant Application Review Committee, The Foundation for Science and Handicapped.

\section{Chair}

- Coordinating Committee on Equal Opportunities in Statistics, American Statistical Association.

Contributing editor

- Current Index to Statistics

President elect

- The Foundation for Science and Handicapped.

\section{Grant}

- Indo-U.S.-Japan Conference, National Science Foundation

\section{Reviewer}

- Science, American Association for the Advancement of Science

- Research Proposal, National Science Foundation

\section{Referee}

- The Annals of the Institute of Statistical Mathematics

- Communications in Statistics

\section{Invited lecture}

- "Discrete Pearson Distribution, College of Engineering, Iowa State University, Ames, IA, April 27, 1992.

\section{R. J. Carter}

\section{Member}

- Human Factors and Ergonomics Society Publications Committee.

- DOE Human Factors Standards Steering Committee.

- Intelligent Vehicle Highway Society of America Safety and Human Factors Committee.

- ANS Human Factors Topical Meeting Technical Program Committee.

- Human Factors and Ergonomics Society Best Technical Group Newsletter Award Committee. 


\section{Liaison}

- Human Factors and Ergonomics Society, ANS Human Factors Topical Meeting.

\section{Chair}

- Human Factors and Ergonomics Society Best Bulletin Article Award Committee.

-- Human Factors and Ergonomics Society Annual Meeting Local Organizing Committee.

\section{Editor}

- Human Factors and Ergonomics Society Bulletin

\section{J. K. Dickens}

\section{General chairman}

- International Conference on Nuclear Data for Science and Technology, Gatlinburg, TN, May 9-13, 1994.

\section{Member}

- American Nuclear Society Standards Working Group 5.1, Decay Heat Standard.

- American Nuclear Society Standards Working Group 19.8, Fission Product Yields.

- American Nuclear Society Standards Working Group 19.9, Delayed Neutrons Standard.

- Nuclear Energy Agency Task Force on Decay Heat Predictions.

- Oak Ridge National Laboratory Speakers' Bureau.

\section{Correspondent}

- Designated by IAEA Working Group on Fission Product Nuclear Data as correspondent on worldwide experimental measurements of fission product decay heat (1977-continuing).

\section{Referee}

- Nuclear Science and Engineering

- Atomic Data and Nuclear Data Tables

- Nuclear Instruments and Methods

- International Journal of Radiation Applications and Instrumentation Part A

- ORNL Fifty-Year History

- U.S. Department of Energy 


\section{Invited lecture}

- "Research with Neutrons at the Oak Ridge Electron Linear Accelerator (ORELA) Facility," Department of Physics, Lowell University, Lowell, MA, April 22, 1992.

\section{Invited papers}

- "White Source Gamma-Ray Production Spectral Measurement Facilities in the U.S.," (with D. C. Larson, R. O. Nelson, and S. A. Wender) 422-426 in Proc. of International Conference on Nuclear Data for Science and Technology, Julich, Germany, May 13-17, 1991 Technology, Julich, Germany, May 13-17, 1991.

- "Scintillation Detector Efficiencies for Neutrons, in the Energy Region above $10 \mathrm{MeV}$," p. 142 in Proc. of a Specialists' Meeting on Neutron Cross Section Standards for the Energy Region Above $20 \mathrm{MeV}$, Uppsala, Sweden, May 21-23, 1991.

\section{F. C. Difilippo}

\section{Member}

- National and Mathematics Division Program Committees of the American Nuclear Society.

\section{Fellow}

- American Nuclear Society

\section{Listed}

- International Directory of Nuclear Criticality Safety Personnel.

\section{Special assignment}

- DOE Technical Representative Gas Cooled Reactor Program at Paul Scherrer Institute, Villigen, Switzerland, M y 6 - November $6,1991$.

\section{Academic}

- Adjunct Professor, Department of Nuclear Engineering, University of Tennessee, Knoxville, TN.

\section{Referee}

- Nuclear Science and Engineering

\section{Invited lecture}

- "Influence of the Angular Correlation of Fission Neutrons on Noise Signatures," 1991 Winter Meeting of the American Nuclear Society, November 1991. 


\section{J. M. Donato}

\section{Chairperson}

- Chairperson of Minisymposium on Numerical Methods for Partial Differential Equations, ICIAM '91 Conference, Washington, DC, July 1991.

\section{Invited lecture}

- "Comparison of Iterative Methods for Nonsymmetric Coupled Elliptic Equations," Institute for Mathematics and Its Applications, Minneapolis, MN, June 1992.

W. W. Engle, Jr.

Fellow

- American Nuclear Society

Executive committee member

- Radiation Protection and Shielding Division, American Nuclear Society

Honors and awards committee member

- Radiation Protection and Shielding Division, American Nuclear Society

D. M. Flanagan

Invited talk

- "The Statistics Partner in Waste Management," Society for Risk Analysis, Risk Methods Day,

E. L. Frome

Member

- ORAU/University of North Carolina Research Planning Group for DOE Health and Mortality Studies.

- Information Systems Working Group for the DOE Comprehensive Epidemiologic Data Resource.

Associate professor

- Department of Biostatistics, University of North Carolina, Chapel Hill, NC.

Associate editor

- The American Statistician, 1983-present.

Statistical Consultant

- Radiation Research Society. 


\section{Referee}

- The American Journal of Epidemiology

- The American Statistician

- Radiation Research

C. Y. Fu

\section{Chairman}

- Subgroup 1, NEANSC International Nuclear Data Evaluation Working Group.

\section{Member}

- Solenoidal Detector Collaboration for the Superconducting Super Collider.

- Subgroup 2, NEANSC International Nuclear Data Evaluation Working Group.

- NEANSC Activation Cross Section Working Group.

- USA Cross Section Evaluation Working Group (CSEWG).

\section{Referee}

- Nuclear Science and Engineering

\section{Invited lecture}

- "Resolving the Calculational Differences Among the EFF-2, ENDF/B-VI, and JENDL-3 Evaluations for the Ni-58 $(n, \alpha)$ Cross Sections," IAEA Coordinated Research Programme on Improvement of Measurements, Theoretical Computations and Evaluations of Neutron-Induced Helium-Production Cross Sections, Debrecen, Hungary, November 17-19, 1992.

\section{Invited paper}

- "Equilibrium and Pre-Equilibrium Models for Calculation of Particle Emission Cross Sections and Spectra," Symposium on Nuclear Data Evaluation Methodology, Brookhaven National Laboratory, Upton, NY, October 12-16, 1992.

T. A. Gabriel

\section{Director}

- Oak Ridge Detector Center

\section{Member}

- Southern Association for High Energy Physics (SAHEP) Executive Committee.

- SAHEP Steering Committee. 
- Solenoidal Detector Collaboration (SDC) Institutional Board.

- IARPE Institutional Board.

\section{Co-initiative manager}

- High Energy Physics Research

\section{Conference session organizer}

- Transport Code Session for the Accelerator Radiation Environmental Workshop, Sante Fe, NM, January 11-15, 1993.

- Detector Session for the MC-93 Conference, Tallahassee, FL, February 22-26, 1993.

- Detector Session for the International Industrial Symposium on the SSC, San Francisco, CA., May 6-8, 1993.

\section{Organizing Committee}

- Member of the Organizing Committee on Accelerator Radiation Environment Workshop, Sante Fe, NM, January 11-15, 1993.

- Member of the Organizing Committee on International Conference on Monte Carlo Simulations in High Energy and Nuclear Physics, Tallahassee, FL, February 22-26, 1993.

- Member of the Planning Team for "How to do Business with the SSCL," Oak Ridge, TN, February 11, 1992, and Co-chairman of the Program Committee.

- Member of the Organizing Committee on the Project, the Progress, The Physics, and Session Chairman, October 17-??, 1992, Corpus Christi, TX.

- Detector Session for the MC-93 Conference, Tallahassee, FL, February 22-26, 1993.

\section{Invited lectures}

- "Detector for the SSC," seminar at the University of Tennessee, Department of Nuclear and Mechanical Engineering, June 8, 1992.

- "The SSC Project," presented at the Local Chapter of the IEEE, Oak Ridge, TN, October 10, 1992.

\section{Invited papers}

- "The Computational Challenges Provided by the Codes Used to Design and Analyze Superconducting Super Collider Detectors," Workshop on Undergraduate and Graduate Education, Louisiana State University, Baton Rouge, LA, April 29-30, 1992.

- "Radiation Levels in and Around the Experimental Halls at the SSCL," presented at the SSC Laboratory on July 5, 1992. 
G. A. Geist

Member

- NAC Committee

- SIAM Society

Subcommittee chairman

- MBI Standards

\section{Invited talks}

- "The PVM System: Supercomputer Level Concurrent Computation on a Network of IBM RS/6000 Powerstations," IBM Large Scale Computer Analysis and Modeling Conference, Yorktown, NY, April 24-26, 1991.

- "Calculating the Eigenvalues and Eigenvectors for a General Matrix Reduced to Tridiagonal Form," Fourth SIAM Conference on Applied Linear Algebra, Minneapolis, MN, September 11-14, 1991.

- "Calculating the Eigenvalues and Eigenvectors for a General Matrix Reduced to Tridiagonal Form, University of Tennessee Minisymposium, Knoxville, TN, September 27, 1991.

- "PVM and HeNCE: A Software Environment for Programming Distributed Heterogeneous Computers," Heterogeneous NetworkBased Concurrent Computing Workshop, Tallahassee, FL, October 16-18, 1991.

- "The PVM System for Distributed Heterogeneous Computing," Distributed Computing Workshop, University of Utah, Salt Lake City, UT, November 14-15, 1991.

- "Using the PVM System: A Case Study," Distributed Computing Workshop, University of Utah, Salt Lake City, UT, November 14$15,1991$.

- "Parallel Superconductor Code Exceeds 2.5 Gflops on iPSC/860," Supercomputer ' 91 , Albuquerque, NM, November 18-22, 1991.

- "PVM and HeNCE: Software for Distributed Computing," Supercomputer ' 91 , Albuquerque, NM, November 18-22, 1991.

- "Getting High Performance Through Parallel Programming," Alabama Supercomputer Center Seminar Series, Huntsville, AL, January 30, 1992.

- "Distributed Computing of the Electronic Structure of Disordered Alloys," Material Modeling Conference, Morganton, WV, April 12-14, 1992.

- "HeNCE: Graphical Development Tools for Network-Based Concurrent Computing," SHPCC 92 Conference, Williamsburg, VA, April 26-30, 1992.

- "PVM Overview," Los Alamos High Performance Computing Workshop, Albuquerque, NM, June 22, 1992. 
- "PVM, HeNCE, and XAB: Graphical Development Tools for Network-Based Concurrent Computing," IBM Europe Institute Workshop on Highly Parallel Computing, Oberlech, Austria, July 13-17, 1992,

- "Solving Material Science Problems at ORNL's Center for Computational Science," SIAM 40th Anniversary Meeting, Los Angeles, CA, July 20-24, 1992.

- "Creation off a Nation-Wide Distributed Computer," Gordon Conference, Plymouth, NH, July 27-31, 1992.

- "PVM 3.0 and the Convex Meta-Series," Supercomputing '92 Convex Booth, Minneapolis, MN, November 18, 1992.

- "PVM: Portable Distributed Computing Software," Supercomputing '92, Minneapolis, MN, November 17, 1992.

- "PVM 3.0 Integrating Multiple Paragons into a Virtual Machine," Supercomputing ' 92 Intel Booth, Minneapolis, MN, November 1719, 1992.

- "PVM 3.0 Overview and Tutorial," Supercomputing '92, Minneapolis, MN, November 20, 1992.

\section{Invited papers}

- "Heterogeneous Network Computing," (with A. Beguelin, J. J. Dongarra, R. Manchek, and V. Sunderam) Supercomputer Review 4(9), 44 (1991).

- "HeNCE: Graphical Development Tools for Network-Based Concurrent Computing," (with A. Beguelin, J. J. Dongarra, R. Manchek, K. Moore, R. Wade, and V. Sunderam) SHPCC '92 Conference, Williamsburg, VA, April 26-30, 1992.

- "Parallel Superconductor Code Exceeds 2.5 Gflops on iPSC/860," Journal of Supercomputing.

C. W. Glover

Member

- Program Committee for the 1993 IEEE International Conference on Neural Networks.

\section{Invited lecture}

- "Neural Network Research at ORNL for High Speed Event Discrimination in Physics Detector Systems," Second International Workshop on Software Engineering, Artificial Intelligence and Expert Systems in High Energy and Nuclear Physics, La Londeles-Maures, France, January 13-18, 1992.

\section{J. Gray}

\section{Member}

- Scientific Organizing Committee for the 15th Boundary Element International Conference, Worchester, MA, August 10-12, 1993. 
- Scientific Organizing Committee for the 8th International Conference on Boundary Element Technology, Vilamoura, Portugal, November 9-11, 1993.

\section{Committee}

- ORNL Awards Night Publication Committee.

- Science Alliance Faculty Awards Committee.

\section{Editorial board}

- Engineering Analysis with Boundary Elements.

- Boundary Elements: Abstracts and Newsletters.

\section{Referee}

- International Journal for Numerical Methods in Engineering

- SIAM J. Applied Mathematics

\section{Invited lectures}

- "Application of Hypersingular Boundary Integral Equations," Clarkson University, Potsdam, NY, February 9, 1992.

- "Hermite Interpolation for Hypersingular Boundary Integrals," Cornell University, Ithaca, NY, February 11, 1992.

- "Simulation of 3D Non-Planar Fatigue Crack Growth in a Turbine Blade Root," (with D. O. Potyondy and A. Ingraffea) ASME Pressure Vessels and Piping 1992 Summer Conference, New Orleans, LA, June 21-25, 1992.

\section{Invited papers}

- "Evaluation of Hypersingular Integrals in the Boundary Element Method," Mathematical and Computer Modelling 15, 165 (1991).

- "Symbolic Computation of Hypersingular Boundary Integrals," Advances in Boundary Element Techniques, Springer Verlag.

- "Hypersingular Integral Evaluation Using Maple," Maple Newsletter, published by Waterloo Maple Software.

\section{M. Haaland}

\section{Advisor}

- National Council for Radiation Protection and Measurements Scientific Committee \#63, Radiation Exposure Control in a Nuclear Emergency, Lewis V. Spencer, Chairman.

\section{Member}

- American Physical Society

- Institute of Electrical and Electronics Engineers

- Sigma Xi 
Referee

- Journal of Civil Defense

- Journal of Health Physics

J. A. Harvey

Member

- ORNL Accelerators and Radiation Sources Review Committee.

- Committee on Membership, American Physical Society.

Labor coordinator

- Engineering Physics and Mathematics Division.

\section{Chairman}

- Division Safety Committee.

Alternate safety officer

- Engineering Physics and Mathematics Division.

Alternate radiation control officer

- Engineering Physics and Mathematics Division.

Referee

- Physical Review C

- Physical Review Letters

- Nuclear Science and Engineering

D. T. Ingersoll

Member

- ORNL Nuclear Energy Panel.

- DOE/SEI Life Support Working Group.

- ORNL Committee on Research Reactor Restart.

- Advisory Committee for International Conference on Nuclear Data for Science and Technology.

- Program Committee for New Horizons in Radiation Protection and Shielding Topical Conference.

\section{Chairman}

- Radiation Protection and Shielding Division, American Nuclear Society.

- Shielding Data Testing Subcommittee, Cross-Section Evaluation Working Group (CSEWG). 
- U.S.-JASPER Shielding Program Working Group.

- ANS Radiation Protection and Shielding Division Nominations Committee.

Reviewer

- DOE Nuclear Engineering Research Program.

- Nuclear Technology Journal.

Invited paper

- "The Horizon for Advanced Reactor Shielding Technology: Sunrise or Sunset?" ANS Topical Meeting on New Horizons in Radiation Protection and Shielding, Pasco, WA, April 26-May 1, 1992.

\section{Award}

- ANS Radiation Protection and Shielding Division Distinguished Service Award.

\section{J. O. Johnson}

\section{Member}

- American Nuclear Society Radiation Protection and Shielding Division Technical Program Committee.

\section{Invited lectures}

- "MASH 1.0 - A Monte Carlo Adjoint Shielding Code System," Defense Nuclear Agency Radiation Environments Program Review Meeting, Munster, Germany, July 28-29, 1992.

- "Application of the MASH 1.0 Code System Armored Vehicle Nuclear Vulnerability Analyses," Defense Nuclear Agency Radiation Environments Program Review Meeting, Munster, Germany, July 28-29, 1992.

- Resolution of Dose and Reduction Factor Questions in APRF Experiments: Renormalization of Calculated and Measured Data," Defense Nuclear Agency Radiation Environments Program Review Meeting, Munster, Germany, July 28-29, 1992.

\section{B. L. Kirk}

\section{Member}

- American Nuclear Society (ANS-10 Standards Committee).

- American Nuclear Society, Professional Women in ANS.

- American Nuclear Society, Program Committee, Math and Computation Division.

- American Nuclear Society/Radiation Protection and Shielding Division Technical Program Committee PASCO Topical Conference 1992. 
- American Nuclear Society/Radiation Protection and Shielding Division Technical Program Committee for 1994 International Topical.

\section{Treasurer}

- American Nuclear Society, Radiation Protection and Shielding Division.

\section{Secretary}

- American Nuclear Society, Oak Ridge/Knoxville Chapter.

H. E. Knee

Member

- Executive Committee: Human Factors Division of the American Nuclear Society.

- American Nuclear Society.

- Smoky Mountain Chapter of the Human Factors and Ergonomics Society.

Technical chairman

- ANS Topical Meeting on Nuclear Plant Instrumentation, Control and Man-Machine Interface Technologies, Oak Ridge, TN, April 18-21, 1993.

\section{Proposal reviewer}

- Federal Highway Administration.

\section{Referee}

- Nuclear Safety Journal

Invited panel speaker

- IEEE Conference on Human Factors and Nuclear Power, June 1992.

\section{Session organizer}

- Human/Machine Interface Session, Fifth Topical Meeting on Robotics and Remote Handling, Knoxville, TN, April 26-29, 1993.

\section{C. Larson}

\section{Member}

- Department of Energy Nuclear Data Committee.

- NEANDC International Task Force on Evaluation Cooperation.

- Evaluation Methods Subcommittee, Cross Section Evaluation Working Group (CSEWG).

- Resonance Region Subcommittee, CSEWG. 


\section{Chairman}

- Working Group A, Office of Nuclear Physics Committee on Nuclear Data for Fusion Reactor Applications.

- Working Group 2, IAEA Specialist's Meetings on Fusion Evaluated Nuclear Data Library.

- Co-chairman of General Purpose Evaluations Subcommittee, CSEWG.

\section{Proposal reviewer}

- Office of Nuclear Physics, Department of Energy, Low Energy Nuclear Physics.

\section{Referee}

- Nuclear Science and Engineering

\section{Invited lectures}

- "Nitrogen Cross-Section Measurements at ORNL," National Research Council's Subcommittee on A-Bomb Dosimetry, Washington, DC, July 25, 1991.

- "Potential Improvements to ENDF/B-VI for Structural Materials," (with C. Y. Fu) International Atomic Energy Agency Advisory Group Meeting on FENDL-2 and Associated Benchmark Calculations, Vienna, Austria, November 18-22, 1991.

- "Status Report to the NEANSC Working Group on International Evaluation Cooperation from Subgroup II: Comparison of the Resonance Regions for ${ }^{52} \mathrm{Cr},{ }^{56} \mathrm{Fe}$, and ${ }^{58} \mathrm{Ni}$," Fourth Meeting of the NEANSC Working Group on International Evaluation Cooperation, Mito, Japan, May 28-29, 1992.

\section{Invited papers}

- "White Source Gamma-Ray Production Spectral Measurement Facilities in the U.S.A." (with J. K. Dickens, R. O. Nelson, and S. A. Wender) pp. 422-426 in Proc. of International Conference on Nuclear Data for Science and Technology, Julich, Germany, May 13-17, 1991.

- "Review of Fission Product Capture Measurements at the Oak Ridge Electron Linear Accelerator (ORELA)," (with R. L. Macklin) The NEANSC Specialists' Meeting on Fission Product Nuclear Data, JAERI Tokai Research Establishment, Ibaraki-Ken, Japan, May 28-29, 1992.

- "Cross Sections of Structural and Shielding Materials," Trans. Am. Nucl. Soc. 66, 149-150 (1992).

- "Approximate Methods for Generation of Covariance Data for the Structural Materials of ENDF/B-VI," (with D. M. Hetrick and C. Y. Fu) Nuclear Energy Agency Nuclear Science Committee Specialist's Meeting on Evaluation and Processing Covariance Data, Oak Ridge, TN, October 7-9, 1992. 
E. Leach

Award

- DOE Award of Excellence.

\section{R. A. Lillie}

\section{Chairman}

- ORNL Reactor Experiments Review Committee.

Referee

- Nuclear Technology

- Nuclear Technology/Fusion

- Journal of Fusion Energy

- Nuclear Science and Engineering

- Nuclear Instruments and Methods

R. E. Maerker

Member

- ANS Standards Committee 19.10.

- Ad-Hoc Committee to Prepare NRC Regulatory Guide on LWRRPV Surveillance Dosimetry Analysis.

Referee

- Nuclear Science and Engineering

- Nuclear Technology

\section{Invited paper}

- "Improvements in Calculational Techniques in Pressure Vessel Dosimetry," (with M. L. Williams) Trans. Am. Nucl. Soc. 64, 489 (1991).

R. C. Mann

Member

- Analysis Simulation and Computer Integration Technology Area Coordination Team - AMTEX Partnership.

\section{Chairman}

- DOE SEI Robotics Working Group.

Assistant program chairman

- ANS Executive Conference on Robotics and Remote Systems in the Nuclear Industry-II, Calloway Gardens, GA, April 5-8, 1992. 


\section{Reviewer}

- IEEE Trans. Systems, Man, Cybernatics

- IEEE Computer

- IEEE J. Robotics \& Automation

- DOE SBIR Program

\section{Invited lectures}

- "Robotics and Related Intelligent Systems for the Space Exploration Initiative," 1992 WATTec Conference, Knoxville, TN, February 19, 1992.

- "Robotics and Intelligent Systems Research at ORNL/CESAR," The Systems Research Center, University of Maryland, College Park, MD, March 27, 1992.

- "Real-Time Qualitative Reasoning and Communication Technologies for Tele-Robotic Systems," Rover/Mobility Systems Workshop, Johnson Space Center, Houston, TX, April 29-30, 1992.

- "High-Speed Pattern Recognition and Analysis of DNA Sequences," (with E. C. Uberbacher, J. R. Einstein, and X. Guan) Institute of Chemical Research, University of Kyoto, Kyoto, Japan, May 22, 1992.

- "CESAR Robotics and Intelligent Systems Research for Nuclear Environments," AIR '92 Conference, Ibaraki-ken, Japan, May 2527, 1992.

- "DOE Basic Research in Intelligent Machines," ANS Executive Conference on Robotics and Remote Systems in the Nuclear Industry-II, Calloway Gardens, Pine Mountain, GA, April 5-8, 1992.

\section{T. J. Mitchell}

\section{Invited papers}

- "Using Computer Experiments to Construct a Cheap Substitute for an Expensive Simulation Model," (with M. D. Morris) Proc. pp. 272-277 of Computing Science and Statistics: Interface 1991, Seattle, WA, April 22-24, 1991.

- "Bayesian Design and Analysis of Computer Experiments: Two Examples," (with M. D. Morris) Statistica Sinica 2, 359-379 (1992).

- "Exploratory Designs for Computational Experiments," (with M. D. Morris) IMS Special Topics Meeting on Industrial Statistics, Philadelphia, PA, June 10-12, 1991.

- "Bayesian Design and Analysis of Computer Experiments: Use of Derivatives in Surface Prediction," (with M. D. Morris and D. Ylvisaker) 1991 Joint Statistical Meeting, Atlanta, GA, August 19-22, 1991. 
- "The Spatial Correlation Function Approach to Response Surface Estimation," (with M. D. Morris) Proc. pp. 565-571 of the 1992 Winter Simulation Conference, Arlington, VA, December 13-16, 1992.

M. D. Morris

Associate editor

- Technometrics

Editorial consultant statistician

- Radiation Research

Invited lectures

- "On Counting the Number of Data Pairs for Semivariogram Estimation," Department of Statistics, Virginia Polytechnic Institute and State University, Blacksburg, VA, April 11, 1991.

- "Design and Analysis for an Inverse Problem of AdvectiveDispersive Fluid Flow," (with A. D. Solomon) Mathematical Sciences Research Institute, Berkeley, CA, May 7, 1992.

- "Exploratory Designs for Computational Experiments," (with T. J. Mitchell) Institute of Mathematical Statistics Special Topics Meeting on Statistics in Industry, Philadelphia, PA, June 9-12, 1991.

\section{F. J. Muckenthaler}

\section{Invited paper}

- "Where Have the Neutrons Gone - A History of the Tower Shielding Facility," American Nuclear Society Winter Meeting, Chicago, IL, November 15-19, 1992.

\section{E. $\mathbf{N g}$}

\section{Invited lectures}

- "Parallel Solution of Sparse Symmetric Positive Definite Linear Systems," Conference on Numerical Methods in Engineering Problems," Caracas, Venezuela, July 15-17, 1991.

- "Direct Solution of Sparse Linear Systems," Department of Computer Science, Universidad Central de Venezuela, Caracas, Venezuela, July 19-26, 1991.

- "Sparse Cholesky Factorization Algorithms," Minisymposium on Direct Sparse Methods, SIAM Conference on Applied Linear Algebra, Minneapolis, MN, September 11-14, 1991.

- "On the Structure of Sparse Gaussian Elimination with Partial Pivoting," IMA Workshop on Sparse Matrix Computations: Graph Theory, Issues, and Algorithms, Minneapolis, MN, October 14-18, 1991. 
- "Solving Linear Systems on Multiprocessor Systems," Department of Computer Science, Radford University, Radford, VA, April 20, 1992.

- "Parallel Sparse Cholesky Factorization Algorithms for SharedMemory Multiprocessor Systems," 7th IMACS International Conference on Computer Methods for Partial Differential Equations, New Brunswick, NJ, June 22-24, 1992.

\section{Invited papers}

- "Parallel Algorithms for Sparse Linear Systems," (with M. Heath and B. Peyton) SIAM Review 33, 420-460 (1991).

- "Experiments with an Ordinary Differential Equation Solver in the Parallel Solution of Method of Lines Problems on a SharedMemory Parallel Computer, Part II," (with D. K. Kahaner, W. E. Schiesser, and S. Thompson) to appear in IMACS Proceedings.

- "Parallel Sparse Cholesky Factorization Algorithms for SharedMemory Multiprocessor Systems," (with E. Rothberg, A. Gupta, and B. W. Peyton) to appear in IMACS Proceedings.

\section{G. Ostrouchov}

\section{Associate editor}

- Journal of Statistical Computation and Simulation

\section{J. V. Pace, III}

\section{Member}

- Working Group on the Reassessment of A-Bomb Dosimetry of the U.S. DOE.

- DOE Terhnical Review Group (TRG) for the Reactor Spent Fuels Cask Systems Development Program (CSDP).

\section{F. G. Pin}

\section{Chairman}

- Robotics Technical Program, ISRAM '96, International Symposium on Robotics and Manufacturing (1996).

- Robotics and Intelligent Systems Technical Program, International Conference on Industrial and Engineering Applications of Artificial Intelligence and Expert Systems.

\section{Co-chairman}

- Robotics Techrical Program, ISRAM '94, International Symposium on Robotics and Manufacturing.

\section{Editorial board}

- International Journal of Robotics and Mechatronics

- Japanese Journal of Advanced Automation Technology 
- Int. J. of Environmentally Conscious Manufacturing

- J. of Intelligent and Fuzzy Systems

Guest editor

- Robotics and Autonomous Systems

Advisory board member

- International Conference on Fuzzy Theory and Technology.

\section{Organizing committee member}

- International Symposium on Methodologies for Intelligent Systems.

- International Conference on Advanced Robotics.

- International Conference on Fuzzy Control and Intelligent Systems.

- International Symposium on Robotics and Manufacturing.

- International Conference on Intelligent Robots and Systems.

\section{Program committee member}

- International Symposium on Automative Technology and Automotion.

- International Congress on Environmentally Conscious Manufacturing.

- International Conference on Intelligent Teleoperation.

- International Symposium on Robotics on Manufacturing.

- International Symposium on Methodologies for Intelligent Systems.

- International Conference on Advanced Robotics.

- International Conference on Intelligent Robots and Systems.

Referee

- IEEE J. of R\&A

- IEEE Trans. on SMC

- IEEE Trans. on Aut. Control

- J. of Rob. Syst.

- J. of Rob. and Auton. Syst.

- Computers and Elect. Eng.

- NSF, DOE-BES, and SBIR Proposals

Member

- IEEE Robotics and Automation Society

- IEEE Systems, Man, and Cybernetics Society 
- IEEE Vehicular Technology Society

- American Mathematical Society

Invited lectures

- "Real Time Qualitative Reasoning for Telerobotic Systems," SOAR '92 Space Operations, Applications, and Research Symposium, Johnson Space Center, Houston, TX, August 4-6, 1992.

- "Application of Custom-Designed VLSI Fuzzy Inferencing Hardware to the Sensor-Based Navigation of an Autonomous Mobile Robot," (with H. Watanabe) International Workshop on Industrial Applications of Fuzzy Control and Intelligent Systems, College Station, TX, November 21-22, 1991.

- "Man-Machine Synergy," International Conference on Intelligent Teleoperation, Greensboro, NC, November 6-7, 1991.

- "Driving a Car with Custom-Designed Fuzzy Inferencing VLSI Chips and Boards," (with Y. Watanabe) International Joint Technology Workshop on Fuzzy Logic and Neural Networks, Houston, TX, June 1-3, 1992.

\section{Honors and awards}

- MMES Special Achievement Award

- MMES President's Award

- Listed in Who's Who in Science and Engineering (1992)

- Listed in Who's Who in America, 47th Edition (1992)

\section{Patent}

- "Omnidirectional and Holonomic Rolling Platform with Decoupled Rotational and Translational Degrees of Freedom" (with S. M. Killough) patent filed November 4, 1992.

\section{R. T. Primm, III}

\section{Chairman}

- Technical Program Chairman, 1994 Reactor Physics Topical Meeting.

- Vice Chairman, Local Section, American Nuclear Society.

\section{Referee}

- Nuclear Science and Technology

\section{Invited lecture}

- "Validation Studies Related to the Storage of Waste from Nuclear Fuel Services in Building 3019 at Oak Ridge National Laboratory," Nuclear Criticality Safety Workshop, Breckenridge, CO, August 27-28, 1992. 
Invited paper

- "Prediction and Measurement of Fuel Depletion Due to Irradiation of HFIR Fuel Elements," American Nuclear Society, Boston, MA, June 7-12, 1992.

\section{A. Protopopescu}

\section{Member}

- Editorial Board of the CRC Mathematical Modelling Series.

- Editorial Board of the Journal Mathematical Methods and Models in Applied Sciences.

- Editorial Board of the Journal Transport Theory and Statistical Physics.

Adjunct professor

- Mathematics Department, University of Tennessee, Knoxville, TN.

\section{Co-organizer}

- Special Session on Competitive Systems at the 2nd International Conference on Industrial and Applied Math, Washington, DC, July 8-12, 1991.

\section{Reviewer}

- Mathematical Reviews

\section{Referee}

- Journal of Statistical Physics

- Transport Theory and Statistical Physics

- Mathematical Methods and Models in Applied Sciences

- Journal of Physics

- IEEE Trans. on Control and Applications

\section{Invited lectures}

- "Modeling Conflict Using Partial Differential Equations," Joint TIMS/ORSA National Meeting, Nashville, TN, May 12-15, 1991.

- "Propagators for Nonlinear Systems," Texas Tech University, Lubbock, TX, January 24, 1992.

- "Linear Chaos: A Mathematical Artifact?" University of Florida at Gainesville, FL, December 9, 1992. 
D. B. Reister

Patent

- Invention disclosure filed by D. B. Reister and M. A. Unseren, "Method for Controlling a Vehicle with Two or Four Independently Steered Wheels," February 1992. Elected by Tech Transfer in March 1992.

R. W. Roussin

Member

- ANS 6.1.2: Shielding Cross Sections Standards Committee.

- Shielding Data Testing and Applications Subcommittee, Cross Section Evaluation Working Group (CSEWG).

- ORNL Federal Credit Union Board of Directors.

- Executive Committee, CSEWG.

International liaison

- 8th International Conference on Radiation Shielding, Arlington, TX.

\section{Chairman}

- Methods and Formats Committee, Cross Section Evaluation Working Group (CSEWG).

- Photon Interaction Evaluation Subcommittee, CSEWG.

- Subgroup 7, Cross Section Processing, NEANDC/NEACRP Committee on International Evaluation Cooperation.

\section{Executive committee}

- American Nuclear Society (ANS) Radiation Protection and Shielding Division (RPSD).

\section{Referee}

- Nuclear Science and Engineering

- Nuclear Technology/Fusion

- Trans. of Symposia on Space Nuclear Power Systems

\section{Invited paper}

- "Activities of the Radiation Shielding Information Center and Report on Codes/Data for High Energy Radiation Transport," Sixth Seminar on Software Development in Nuclear Energy Research, Tokai Mura, Japan. 


\section{Award}

- American Nuclear Society, Radiation Protection and Shielding Division, Distinguished Service Award.

\section{T. H. Rowan}

\section{Member}

- SLATEC Common Mathematical Library Subcommittee.

- HPCC National Software Exchange Technical Working Group.

\section{R. T. Santoro}

\section{Member}

- ORNL Enterprise Committee.

- American Nuclear Society, Honors and Awards Committee.

- American Nuclear Society, Radiation Protection and Shielding Division Executive Committee.

\section{Referee}

- Fusion Technology

- Nuclear Science and Engineering

- Nuclear Technology/Fusion

\section{Invited papers}

- "Review of Experiments for Benchmarking MASH - Version 1.0: Discussion of Measurements and Analyses," (with S. Y. Whitaker) Radiation Environments Program Review," Munster, Germany, July 28-29, 1992.

- "Resolution of Dose and Reduction Factor Questions in APRF Experiments: Renormalization of Calculated and Measured Data," (with J. D. Drischler and J. O. Johnson) Radiation Environments Program Review, Munster, Germany, July 28-29, 1992.

- "Application of the MASH 1.0 Code to Armored Vehicle Nuclear Vulnerability Analyses," (with J. O. Johnson, J. M. Barnes, T. J. Burns, and J. D. Drischler) Radiation Environments Program Review, Munster, Germany, July 28-29, 1992.

- "Overview of the ORNL DNA Radiation Environments Program," Third Annual Radiation Risk/Safety Technology Transfer Meeting, Alexandria, VA, December 1-3, 1992.

- "Two-Meter Box/Phantom Benchmark Experiments, Analyses and Results," (with J. O. Johnson and S. Y. Whitaker) Third Annual Radiation Risk/Safety Technology Transfer Meeting, Alexandria, VA, December 1-3, 1992. 
- "Armored Vehicle Analyses - U.S. and Foreign Systems," (with J. O. Johnson, J. M. Barnes, T. J. Burns, and J. D. Drischler) Third Annual Radiation Risk/Safety Technology Transfer Meeting, Alexandria, VA, December 1-3, 1992.

\author{
M. S. Smith
}

Member

- 1993 WATTec Sponsor's Committee, American Nuclear Society.

\title{
Invited paper
}

- "An Assessment of Liquid Metal Centrifugal Pumps at Three Fast Reactors," Nuclear Technology, September 1992.

D. W. Walker

\section{Member}

- Program Committee of the 7th ACM International Conference on Supercomputing.

- Steering Committee of Perfect Benchmarking Group.

- Organizing Committee for 1992 Scalable High Performance Computing Conference.

\section{Chairman}

- 1992 Gordon Research Conference on Software Tools and Libraries for Concurrent Supercomputer.

\section{Executive director}

- MPI Forum.

\section{Invited lectures}

- Standards for Message Passing on Distributed Memory Computers: The U.S. View," RAPS (Real Application on Parallel Systems) Workshop on Parallel Programming Models and Interfaces, Bonn, Germany, May 4, 1992.

- "Parallelizing the Shallow Water Equation Code," Fifth Workshop on the Use of Parallel Processes in Meterology, November 1992.

\section{Invited paper}

- "The Design of Scalable Software Libraries for Distributed Memory Concurrent Computers," (with J. Choi and J. J. Dongarra) CNRS-NSF Collaboration Workshop on Environments and Tools for Parallel Scientific Computing, St. Hilaire du Touvet, France, September 7-8, 1992. 
R. C. Ward

Member

- College of Engineering Advisory Council, North Carolina State University, 1991-93.

- Advisory Committee, Applied Linear Algebra Year, Institute for Mathematics and Its Applications, University of Minnesota, 199192.

- Advisory Committee, Second International Conference on Computational Physics, Beijing, China, 1993.

- Program Committee, Supercomputing '93, Portland, OR, November 15-19, 1993.

Invited paper

- "A DOE Partnership for Grand Challenge Applications," 1992 American Nuclear Society Topical Meeting on Advances in Reactor Physics, Charleston, SC, March 8-11, 1992.

Proposal reviewer

- National Science Foundation.

- Department of Energy.

\section{Fellow}

- American Association for Advancement of Science.

\section{J. E. White}

Member

- American Nuclear Society 6.2 and 19.1 Standards Committees.

Reviewer

- Nuclear Technology Journal

B. A. Worley

Member

- ANS Reactor Physics Program Committee.

- NPR-MHTGR Integral Data Steering Committee.

- DOE/NASA Technical Committee on System Model Development for Nuclear Thermal Propulsion.

\section{U.S. representative}

- IAEA Coordinated Research Program on Validation of Safety Related Physics Calculations for Gas-Cooled Reactors. 


\section{Technical lead}

- U.S. MHTGR Reactor Physics Program Committee.

\section{T. Wright}

Member

- National Advisory Panel of Experts on Adjustment of 1990 Census Data for Post-Census Estimates, U.S. Bureau of the Census.

- Executive Committee and Publications Officer, Section on Survey Research Methods, American Statistical Association.

- Steering Committee, Tennessee Mathematics Coalition.

- Steering Committee, Tennessee Systemic Initiative, Tennessee Board of Education.

Invited discussant

- 1992 Annual Research Conference, U.S. Bureau of the Census.

Editorial board

- The American Journal of Mathematical and Management Sciences.

Board of directors and treasurer

- Tennessee Mathematics and Computer Sciences Foundation, Inc.

Associate professor

- (Mathematics) Knoxville College, Knoxville, TN.

- (Statistics/Mathematics) University of Tennessee, Knoxville, TN.

\section{Reviewer}

- Mathematical Reviews

\section{Referee}

- International Review

- The American Statistician

- Metrika 


\section{CONFERENCES}

1991

"Workshop on Neural Networks as Applied to Current and Future Detector Systems," May 8-9, 1991, Oak Ridge, TN

Organizer: $\quad$ T. A. Gabriel

Attendees: $\quad 30$

Sponsors: $\quad$ ORNL, Oak Ridge Detector Center

"Solenoidal Detector Collaboration Meeting," September 26-28, 1991, Oak Ridge, TN

Organizer: $\quad$ T. A. Gabriel

Attendees: $\quad 200$

Sponsors: $\quad$ ORNL, Oak Ridge Detector Center

"ISMIS '91 6th International Symposium on Methodologies for Intelligent Systems, October 16-19, 1991, Charlotte, NC

Organizers: $\quad$ F. G. Pin and K. S. Harber

Attendees: $\quad 200$

Sponsor: $\quad$ CESAR

"CHAMMP (Computer Hardware, Advanced Mathematics, and Model Physics) Workshop," November 11-1?, 1991, Oak Ridge, TN

Organizer: $\quad$ J. B. Drake

Attendees: $\quad 20$

Sponsor: Atmospheric Climate Model Project

1992

"DOE/NE Robotics for Advanced heactor First Annual Student Conference," January 16-18, 1992, Oak Ridge, Tennessee

Organizer: $\quad$ A. L. Houston and F. J. Sweeney

Attendees: $\quad 45$

Sponsors: $\quad$ DOE/NE Robotics for Advanced Reactors Program 
"Workshop on Deterministic Methods in Radiation Transport," February 3-6, 1992, Oak Ridge, Tennessee

Organizers: $\quad$ D. T. Ingersoll, W. A. Rhoades, R. W. Roussin, and R. T. Santoro

Attendees: $\quad 90$

Sponsors: Nuclear Analysis and Shielding Section and the Radiation Shielding Information Center

"Workshop for TORT Three-Dimensional Discrete Ordinates Neutron/Photon Transport Code," February 5-6, 1992, Oak Ridge, Tennessee

Organizers: $\quad$ D. T. Ingersoll and R. W. Roussin

Attendees: $\quad 90$

Sponsors: Nuclear Analysis and Shielding Section and the Radiation Shielding Information Center

"J A.SPER Program Review Meeting," April 23-24, 1992, Oak Ridge,
Tennessee

Organizer: $\quad$ J. V. Pace

Attrndees: $\quad 15$

Sṛ:onsor: $\quad$ ORNL, Nuclear Analysis and Shielding Section

"First CRPC Workshop on Standards for Message Passing in a Distributed Memory Environment," April 29-30, 1992, Williamsburg, VA

Organizer: $\quad$ D. W. Walker

Attendees: $\quad 68$

Sponsors: $\quad$ Center for Research on Parallel Computing

"Modeling and Prediction Using Chaotic Time Series Analysis," June 5, 1992, Oak Ridge, Tennessee

Organizers: $\quad$ D. J. Downing, W. F. Lawkins, and M. A. Harris

Attendees: $\quad 60$

Sponsors: ORNL, Mathematical Sciences Section, Engineering Technology Division, and I\&C Division 
"1992 Gordon Research Conference on Software Tools and Libraries for Concurrent Supercomputing," July 27-31, 1992, Plymouth State College, Plymouth, NH

$\begin{array}{ll}\text { Chair: } & \text { D. W. Walker }\end{array}$

Attendees: $\quad 100$

"NEANSC Specialist' Meeting on Evaluation and Processing of Covariance Data," October 7-9, 1992, Oak Ridge, TN

Organizer: $\quad$ R. W. Peelle

Attendees: $\quad 25$

Sponsors: $\quad$ ORNL, OECD, Nuclear Energy Agency Nuclear Science Committee 


\section{SEMINARS AT ORNL}

Y. Y. Azmy was the seminar coordinator during the period covered by this report. The following seminars were held during April 1991 through December 1992.

A. L. Bangs, Engineering Physics and Mathematics Division, "The Mac: 'Everything you Wanted to Know, But Were Afraid to Ask' or 'Everything Your Mother Didn't Tell You"'

J. Barlow, Penn State University, University Park, PA, "Error Analysis of Update Methods for the Symmetric Eigenvalue Problem"

J. S. Bay, Bradley Department of Electrical Engineering, Virginia Polytechnic Institute and State University, Blacksburg, VA, "Differential Geometry in Intelligent Robotics: Pseudoinverse Kinematics and Autonomous Exploration"

A. Beguelin, Engineering Physics and Mathematics Division, "Heterogeneous Network Computing"

V. V. Bolyatko, Moscow Engineering Physics Institute, Moscow, Soviet Union, "Sensitivity and Uncertainty Analysis Applied to the Design of Reactor Shielding"

M. Borodovsky, Georgia Institute of Technology, Atlanta, GA, "Statistical Analysis, Modeling, and Identification of DNA Sequence Signals and Messages"

R. Bramley, Center for Supercomputing Research and Development, University of Illinois, Urbana, IL, "Orthogonal Projection Algorithm for Linear Stokes Problems"

H. Bunke, University of Bern, Switzerland, "Efficient Matching of Dynamically Changing Graphs"

D. Calvetti, Stevens Institute of Technology, Hoboken, NJ, "Adaptive Chebyshev Iterative Methods for Nonsymmetric Linear Systems Based on Modified Moments"

T. F. C. Chan, Department of Mathematics, University of California, Los Angeles, CA, "A Composite Step Biconjugate Gradient Algorithm for Nonsymmetric Linear Systems"

J. Choi, School of Electrical Engineering, Cornell University, Ithaca, NY, "Recursive Least Squares on Linear Array Processors"

H. I. Christensen, Aalborg University, Denmark, “'Vision as Process' Experiences and Results from a European Research Action"

A. E. DePristo, Ames Laboratory, U.S. DOE and Department of Chemistry, Iowa State University, Ames, IA "Theory and Application of the Corrected Effective Medium Method" 
J. Donato, Engineering Physics and Mathematics Division, "The Fourier Analysis Technique and Epsilon-Pseudo-Eigenvalues"

J. Donato, Engineering Physics and Mathematics Division, "Introduction to OneStep Iterative Methods" (first meeting)

J. Donato, Engineering Physics and Mathematics Division, "Introduction to OneStep Iterative Methods" (second meeting)

J. Donato, Engineering Physics and Mathematics Division, "Minimization Methods Such as Steepest Descent and Conjugate Gradient" (third meeting)

L. Dowdy, Department of Computer Science, Vanderbilt University, Nashville, TN, "Performance Modeling of Multiprocessor Systems"

T. Dunigan, Engineering Physics and Mathematics Division, "Architecture and Performance of the Kendall Square Multiprocessor"

B. Fitzpatrick, University of Tennessee, Knoxville, TN, "Bayesian Methods in Identification and Control"

P. A. Forsyth, University of Waterloo, Ontario, "A Positivity Preserving Method for Simulation of Steam Injection for NAPL Site Remediation"

R. Freund, NASA Ames Research Center, "QMR: A Quasi-Minimal Residual Method for the Solution of Non-Hermitian Linear Systems"

K. Gates, ETH (The Wissenschaftliches Rechnen), Zurich, Switzerland, "A Parallel Implementation of the Symmetric Tridiagonal QR Algorithm"

T. A. Germogenova, Keldysh Institute of Applied Mathematics, U.S.S.R. Academy of Science, Moscow, "Adaptive Positive Nodal Method for the Transport Equation in Multidimensional Geometries"

R. M. Haralick, University of Washington, Seattle, WA, "Performance Characterization in Computer Vision"

A. Hogenbirk, The Netherlands Energy Research Foundation, Petten, "Sensitivity and Uncertainty Calculations in Fusion Reactor Shielding Blankets"

T. E. Hutchinson, University of Virginia, Charlottesville, VA, "ERICA-Eyegaze Response Interface Computer Aid"

T. E. Hutchinson, Calcott Professor, University of Virginia, Charlottesville, VA, "Eye-Gaze: Its Potentials as a New Interface Mode and Research Tool"

M. Jamshidi, AT\&T Professor and Director, CAD Laboratory for Systems/ Robotics, Department of Electrical and Computer Engineering, The University of New Mexico, Albuquerque, NM, "Intelligent Control: From Observation to Intuition and Its Potentials in Robotics"

B. D. Jawerth, University of South Carolina, Columbia, SC, "Wavelets and Applications to Compression and Integral Equations" 
M. Johnson, NASA Ames Research Center, Moffett Field, CA, "Using HighPerformance Networks to Enable Supercomputer Applications"

E. Kent, National Institute of Standards and Technology, Washington, DC, "Robots, Continuous Spaces, and Machine Semantics"

P. K. Khosla, Associate Professor and Director, Advanced Manipulators Laboratory, The Robotics Institute, Carnegie Mellon University, Pittsburgh, PA, "The Carnegie Mellon University (CMU) Reconfigurable Modular Manipulator System"

D. R. Kincaid, Center for Numerical Analysis, The University of Texas at Austin, Austin, TX, "ITPACK Software"

K. L. Kliewer, Purdue University, West Lafayette, IN, "CCS: Where Do We Go and How Do We Get There?"

H. E. Knee, Engineering Physics and Mathematics Division, "Models of Human Operators: Their Need and Usefulness for Improvement of Advanced Control"

M. M. Kulas, Department of Nuclear Engineering and Engineering Physics, University of Wisconsin, Madison, WI, "Projected Discrete Ordinates Methods for SN Problems in Two-Dimensional Geometries"

S. L. Lee, University of Illinois at Urbana-Champaign, Urbana, IL, "Krylov Methods for the Solution of Nonlinear Initial Value Problems in ODEs and DAEs"

S. Lenhart, Engineering Physics and Mathematics Division and The University of Tennessee, Knoxville, TN, "Optimal Control of Parabolic PDE Governing Beaver Populations"

S. M. Lenhart, Engineering Physics and Mathematics Division and The University of Tennessee, Knoxville, TN, "Optimal Control of Differential Models Involving Waste Clean-Up"

W. Ligon, Georgia Institute of Technology, Atlanta, GA, "Empirical Evaluation of Architectural Reconfigurability"

N. Lincoln, Supercomputer Systems Engineering and Services Company, Minneapolis, MN, "Supercomputers in the 1990s-the Developer's Dilema"

J. Loskiewicz, Institute of Nuclear Physics, Krakow, Poland, "Computational Work at Krakow Institute of Nuclear Physics on Shielding of High Energy Accelerators"

S. Lundstrum, Stanford University, Stanford, CA, "Microelectronics and Computer Technology Corporation, Research and Technology Briefing"

W. R. Martin, University of Michigan, Ann Arbor, MI, "The Response History of the Monte Carlo Method for Electron Transport" 
A. P. Mills, Jr., AT\&T Bell Laboratories, Murray Hill, NJ, "Basic Physics and Materials Analysi: Experiments with Pulsed Positron and Muon Beams"

N. Morgan, Virginia Polytechnic Institute and State University, Blackburg, VA, "A Report on Resistive Plate Counters for Charged Particle Detection"

J. Myczkowski, Thinking Machines Corporation, Cambridge, MA, "Atmospheric and Ocean Modeling on the Connection Machine"

V. Nair, AT\&T Bell Laboratories, "Quality Improvement in Integrated Circuit Fabrication Through Statistical Analysis of Wafer Maps"

W. S. Newman, Director, Center for Automation and Intelligent Systems Research and Associate Professor, Department of Electrical Engineering and Applied Physics, Case Western Reserve University, "Recent Advances in Robotics Research at Case Western Reserve University"

J. M. Ortega, University of Virginia, Charlottesville, VA, "Solution of Nonlinear Poisson-Type Equations"

S. W. Otto, Department of Computer Science and Engineering, Oregon Graduate Institute, Beaverton, OR, "MetaMP: A Higher-Level Abstraction for Message-Passing Programming"

F. G. Pin, Engineering Physics and Mathematics Division, "Approximate Reasoning for Autonomous Robots Using Custom-Designed VLSI Inferencing Systems"

N. S. V. Rao, Engineering Physics and Mathematics Division, "N-Learners Problem"

V. Rego, Department or Computer Sciences, Purdue University, West Lafayette, IN, and V. S. Sunderam, Department of Mathematics and Computer Science, Emory University, Atlanta, GA, "Experiments in Concurrent Simulation with Eclipse"

L. Reichel, Kent State University, Kent, OH, "Adaptive Iterative Methods for Symmetric Linear Systems of Equations"

E. Rothberg, Stanford University, Stanford, CA, "Exploiting Data Reuse in Sequential and Parallel Sparse Cholesky Factorization"

R. F. Schwitters, Superconducting Super Collider Laboratory, Dallas, TX, "The Super Collider"

M. S. Selig, University of Illinois, Department of Aeronautical and Astronautical Engineering, "Multi-Point Inverse Airfoil and Cascade Design"

B. D. Semeraro, Department of Computer Science, University of Ilinois at UrbanaChampaign, "Time Splitting Methods for the Navier-Stokes Equations"

G. Serazzi, Politecnico di Milano, Dipartimento di Elettronica, Piazza Leonardo da Vinci, Milano, Italy, "Workload Characterization and Scheduling in Multiprogrammed Parallel Systems" 
J. Sethian, University of California, Berkeley, CA, "Computing Turbulent Flow and Massively Parallel Processors: New Algorithms and New Machines"

W. A. Shelton, Jr., Computational Physics, Inc. Annandale, VA, "Development of Parallel Algorithms for Electronic Structure Methods"

R. F. Sincovec, Engineering Physics and Mathematics Division, "Partial Differential Equations Tools for Modeling Physical Systems and a Vision for the Center for Computational Science"

R. Skaalin, Project Manager of Supercomputing, The Norwegian Institute of Technology (NTH) and The Foundation for Scientific and Industrial Research at NTH (SINTEF), Trondheim, Norway, "Supercomputing Activities in Norway"

B. Smith, Chairman of the Board and Chief Scientist, TERA Computer, "Recent Developments in Tera Computer System Concept and Product"

A. D. Solomon, Consultant, Omer, Israel, "Recent Work on the Use of the Stator Current for the Characterization of the Performance of Motor-Driven Equipment"

A. D. Solomon, Consultant, Omer, Israel, "Mathematical Aspects and Qualitative Behavior of Solutions to the Advection-Dispersion Equation in One Space Dimension"

A. D. Solomon, Consultant, Omer, Israel, "The One-Dimensional Advection Dispersion Equation-Qualitative Behavior of Its Solutions"

K. Stephenson, University of Tennessee, Mathematics Department, Knoxville, TN, "Circle Packing: A New Departure in Discrete Structures"

S. B. Trickey, Executive Director, Information Technologies and Services, University of Florida, Gainesville, FL, "Ultra-Thin Calculations and Implications for Computational Strategy"

A. W. Trivelpiece and B. R. Appleton, and members of the Oak Ridge Detector Center Staff, "Current Status of the Superconducting Super Collider"

A. Tsao, Supercomputing Research Center, Bowie, MD, "Matrix Multiply on the Intel Multicomputer"

L. W. Tucker, Thinking Machines Corporation, Cambridge, MA, "CMMD: Message Passing System for the CM-5"

E. Uberbacher, Engineering Physics and Mathematics Division, "A Computational Approach to Gene Recognition and Interpretation in Human Genome DNA Sequence Data"

V. G. Vaidya, Boeing Defense and Space Group and University of Washington, Seattle, WA, "The Use of the Generalized Space Frequency Representation for Object Location Identification and Motion Estimation" 
R. C. Ward, Division Director, Engineering Physics and Mathematics Division, "ORNL Center for Computational Science"

J. W. Wilson, NASA Langley Research Center, Hampton, VA, "Radiation Protection for Deep-Space Missions"

$X$. Yun and V. Kumar, GRASP Laboratory, University of Pennsylvania, Philadelphia, PA, "Coordination of Multi-Agent Robotic Manipulation" 


\section{PUBLICATIONS}

ABLES, E., ${ }^{*}$..., M. L. BAUER, ${ }^{*}$ B. L. BISHOP, ${ }^{*}$ H. O. COHN,* T. A. GABRIEL, A. GORDEEV,* Y. KAMYSHKOV,* R A. LILLIE, F. PLASIL, ${ }^{*}$ K. READ, ${ }^{*}$. J. RENNICH, * A. SAVIN,* K. SHMAKOV, ${ }^{*}$ B. H. SINGELTARY, ${ }^{*}$ A. SMIRNOV,* E. TARKOVSKY, ${ }^{*}$ R A. TODD, ${ }^{*}$ K. G. YOUNG, ${ }^{*}$ et al.

"Liquid Scintillator Calorimetry (Second Year) a Research and Development Renewal Proposal to the Superconducting Super Collider Laboratory," ORNL/M-1774 (September 1991). (4.25) ${ }^{\dagger}$

\section{ALEXIADES, $\mathrm{V}$.}

"Equilibrium Size of a Solid Nucleus Forming in Supercooled Liquid," book chapter in Free Boundary Problems, Vol. 186, pp. 681-684, K.-H. Hoffmann and J. Sprekels, Eds., Longman, New York (1990). (1989)

\section{ALSMILLER, R. G., JR., AND F. S. ALSMILLER}

"Low-Energy Particle Production and Transport for $200 \mathrm{GeV} / \mathrm{c}$ Protons in Iron and Comparisons with Experimental Data," Nucl. Instrum. Methods Phys. Res. A306, 500 (1991). (1991)

ANDERSEN, C. S.,* C. B. MADSEN, J. J. SORENSEN, N. O. S. KIRKEBY,* J. P. JONES, AND H. I. CHRISTENSEN*

"Navigation Using Range Images on a Mobile Robot," Robotics and Autonomous Systems 10, 147 (1992). (1991)

AZMY, Y. Y.

"DCOR: A Deterministic Combat Model Code," ORNL/TM-11690 (April 1991). (4.72)

"Arbitrarily High Order Characteristic Methods for Solving the Neutron Transport Equation," Nuclear Energy 19, 593 (1992). (4.60)

"Performance and Performance Modeling of a Parallel Algorithm for Solving the Neutron Transport Equation," Journal of Supercomputing 6, 211 (1992). (4.62)

\footnotetext{
* Not a member of Engineering Physics and Mathematics Division.

$\dagger$ In most cases. the number shown in parentheses following the publication corresponds to the number of an abstract included in this report. In some cases, the number is a year, which means the abstract was published in an earlier progress report published that year. If neither an abstract number nor a year appears, then an abstract was not available for this report.
} 
AZMY, Y. Y., V. C. BOFFI, V. PROTOPOPESCU, AND J. MANDREKAS*

"Lie Group Analysis for a Multispecies, Spatially Inhomogeneous, Mutually Interacting Gas Mixture," Transport Theory and Statistical Physics 21, 119 (1992). (4.73)

\section{AZMY, Y. Y. AND V. PROTOPOPESCU}

"On the Dynamics of a Discrete Reaction-Diffusion System," Numerical Methods for Partial Differential Equations 7, 385 (1991). (1991)

BAKER, J. E.

"Empirical Characterization of a High Intensity LED Proximity Sensor," ORNL/TM-11972 (November 1991). (3.31)

"Terrain Following of Arbitrary Surfaces Using a High Intensity LED Proximity Sensor," ORNL/TM-11991 (January 1992). (3.32)

BAN, S.,* AND H. HIRAYAMA*

"Shielding Design Methods of a Synchrotron Radiation Facility Part I: Accelerator," ORINL/TR-91/4 (April 1991). (5.7)

BARNES, J. M., * J. O. JOHNSON, T. J. BURNS, AND J. D. DRISCHLER

"Nuclear Vulnerability of the U.S. M60A1 Tank in an Initial Radiation Environment: MASH Code System Analysis," ORNL/TM-11775 (May 1991). (4.43)

\section{BAUER, R. J., JR.,* AND G. E. LIEPINS}

"Genetic Algorithms and Computerized Trading Strategies," book chapter in Expert Systems in Finance, pp. 89-100, Elsevier Science Publishers, North Holland, 1992. (3.40)

BEAUChAMP, J. J., S. W. ChRISTENSEN,* AND E. P. SMITH* "Selection of Factors Affecting the Presence of Brook Trout (Salvelinus fontinalis) in Adirondack Lakes: A Case Study," Can. J. Fish. Aquat. Sci. 49(3), 597 (1992). (1.100)

BEAUCHAMP, J. J., J. F. MCCARTHY, D. H. ROSENBLATT, AND L. R. SHUGART*

"Statistical Design for Sampling and Analysis of Animal Populations for Chemical Contamination," Risk Analysis 12(2), 233 (1992). (1.96)

\section{BECKERMAN, M.}

"Phase I Report on Sensor Technology, Data Fusion and Data Interpretation for Site Characterization," ORNL/TM-11971 (October 1991). (3.33) 
BEGUELIN, A., J. J. DONGARRA, G. A. GEIST, AND R. MANCHEK,* AND V. SUNDERAM*

"Heterogeneous Network Computing," Supercomputer Review 4(9), 44 (1991). (1.4)

"A User's Guide to PVM Parallel Virtual Machine," ORNL/TM-11826 (July 1991). (1.1)

BLAIR, J. R. S., * AND B. W. PEYTON

"On Finding Minimum-Diameter Clique Trees," ORNL/TM-11850 (August 1991). (1.38)

"An Introduction to Chordal Graphs and Clique Trees," ORNL/TM-12203 (November 1992). (1.37)

BLENCOE, J. G.,* M. T. NANEY,* D. J. WESOLOWSKI,* AND F. G. PEREY

"Tests for 'Cold Fusion' in the Pr- $\mathrm{D}_{2}$ Systems at 40 to $380 \mathrm{MPa}$ and -196 to $27^{\circ} \mathrm{C}$," Fusion Energy' 9, 149 (1990). (1989)

BOWMAN, K. O., J. FERNSTERMACHER,* B. BRUSH,* V. STERN,* AND A. B. SWANSON*

"Report of the National Science Foundation Task Force on Persons with Disabilities," ORINL/M-1535 (1991).

BOWMAN, K. O., T. H. HOPP,* R. N. KACKER,* AND R. J. LUNDEGARD*

"Statistical Quality Control Technology in Japan," Chance 4(3), 15 (1991). (1991)

BOWMAN, K. O., M. A. KASTENBAUM,* AND L. R. SHENTON* "The Negative Hypergeometric Distribution and Estimation by Moments," Cominun. Statist. Simula. 21(2), 301 (1992). (1.85)

BOWMAN, K. O., AND L. R. SHENTON*

"Parameter Estimation for the Beta Distribution," J. Siatist. Comput. Simul. 43, 217 (1992). (1.87)

"Some Exact Expressions for Functions of Sample Moments," Annals of the Institute of Statistical Mathematics 44(4), 781 (1992). (1991)

BOWMAN, K. O., L. R. SHENTON,* AND M. A. KASTENBAUM*

"Discrete Pearson Distributions," The Institute of Mathematical Statistics Bulletin 20, 410 (1991); also ORNL/TM-11899 (November 1991). (1.90) 
"Mixtures of Logarithmic Distributions," IMS Bulletin 20, 239 (1991). (1.88)

"Overdispersion: Notes on Discrete Distributions," ORNL/TM-12167 (September 1992). (1.89)

BURNS, T. J.

"CGVIEW - A Program to Generate Isometric and Perspective Views of Combinatorial Geometries," ORNL/TM-12019 (July 1992). (4.65)

CARLSON, B. M.,* T. D. WAGNER,* L. W. DOWDY,* AND P. H. WORLEY

"Speedup Properties of Phases in the Execution Profile of Distributed Parallel Programs," ORNL/TM-11900 (August 1992). (1.5)

\section{CARO, M.,* AND F. C. DIFILIPPO}

"Simulation of the Pulsed Neutron Source Experiment," ORNL/M-2157 (June 1992). (4.2)

CARTER, R. J.

"Human Factors Survey of Advanced Instrumentation and Controls Technologies in Nuclear Plants," Nuclear Engineering and Design 134, 319 (1992). (1991)

CACUCI, D.,* AND V. PROTOPOPESCU

"Canonical Propagators for Nonlinear Systems: Theory and Sample Applications," Lecture Notes in Mathematics 1460, 43 (1991). (1989).

\section{CHATANI, K.*}

"Application of 3-Dimensional Radiation Transport Codes to the Analysis of the CRBR Prototypic Coolant Pipe Chaseway Neutron Streaming Experiment," ORNL/TM-11641 (Auơust 1992). (4.3)

CHIN, P., * E. F. D'AZEVEDO, P. A. FORSYTH,, AND W.-P. TANG* "Preconditioned Conjugate Gradient Methods for the Incompressible NavierStokes Equations," Int. J. Numer. Methods Fluids 15, 273 (1992). (1.47)

CRAMER, S. N., AND F. G. PEREY

"Time Dependent Monte Carlo Calculations of the ORELA Target Neutron Spectrum," Nucl. Sci. Eng. 111, 102 (1992). (5.8)

\section{CULIOLI, J.-C.,* AND G. COHEN*}

"Decomposition/Coordination Algorithms in Stochastic Optimization," SIAM J. Control and Optimization 28(6), 1372 (1990). 
CURRIN, C., ${ }^{*}$ T. J. MITCHELL, M. D. MORRIS, AND D. YLVISAKER*

"Bayesian Prediction of Deterministic Functions, with Applications to the Design and Analysis of Computer Experiments," Journal of the American Statistical Association 86(416), 953 (1991). (1991)

D'AZEVEDO, E. F., P. A. FORSYTH,* AND W.-P. TANG*

"Ordering Methods for Preconditioned Conjugate Gradient Methods Applied to Unstructured Grid Problems," SIAM J. Matrix Anal. Appl. 13(3), 944 (1992). (1991)

"Towards a Cost-Effective ILU Preconditioner with High Level Fill," BIT 32, 447 (1992). (1.63)

"Drop Tolerance Preconditioning for Incompressible Viscous Flow," Int. J. Comp. Math. 44, 301 (1992). (1.48)

D'AZEVEDO, E. F., AND C. H. ROMINE

"Reducing Communication Costs in the Conjugate Gradient Algorithm on Distri')uted Memory Multiprocessors," ORNL/TM-12192 (September 1992). (1.6\%)

D'AZEVEDO, E. F., AND R. B. SIMPSON*

"On Optimal Triangular Meshes for Minimizing the Gradient Error," Numer. Math. 59, 321 (1991). (1991)

DE SAUSSURE, G., N. M. LARSON,* J. A. HARVEY, AND N. W. HILL*

"Multilevel Resonance Analysis of ${ }^{59} \mathrm{Co}$ Neutron Transmission Measurements," ORNL/TM-11762 (May 1991). (2.23)

"Multilevel Resonance Analysis of ${ }^{59} \mathrm{Co}$ Neutron Transmission Measurements," Ann. Nucl. Energy 19(7), 393 (1992). (2.22)

DICKENS, J. K.

"Computed Secondary-Particle Energy Spectra Following Nonelastic Neutron Interactions with ${ }^{12} \mathrm{C}$ for $E_{n}$ Between 15 and $60 \mathrm{MeV}$ : Comparisons of Results from Two Calculational Methods," ORNL/TM-11812 (April 1991). (2.41)

"Analysis of Beta-Ray Data Important to Decay Heat Predictions," Nucl. Sci. Eng. 109, 92 (1991). (1991)

\section{DICKENS, J. K., T. R. ENGLAND,* AND R. E. SCHENTER*}

"Current Status and Proposed Improvements to the ANSI/ANS-5.1 American National Standard for Decay Heat Power in Light Water Reactors," Nuclear Safety 32(2), 209 (1991). (2.43) 
DIFILIPPO, F. C.

"Temperature-Dependent Bondarenko Factors for ${ }^{237} \mathrm{~Np},{ }^{241} \mathrm{Am}$, and ${ }^{243} \mathrm{Am}$," ORNL/TM-11727 (April 1991). (4.9)

"Temperature-Dependent Bondarenko Factors for ${ }^{243} \mathrm{Cm},{ }^{244} \mathrm{Cm}$, and ${ }^{245} \mathrm{Cm}$," ORNL/TM-11837 (May 1991). (4.10)

"Correlation Between Fission Neutrons and the Calculation of Stochastics Descriptors Application to the ${ }^{252} \mathrm{Cf}$ Ratio Method," Annals of Nuclear Energy 18(9), 503 (1991). (1991)

"LEU-HTR PROTEUS: Theory and Simulations of Reactivity Measurements with the Inverse Kinetics Method," ORNL/M-2156 (June 1992). (4.6)

"LEU-HTR PROTEUS: Neutron Kinetics and Procedures to Analyze Pulsed Neutron Experiments," ORNL/M-2154 (June 1992). (4.7)

"PNS Measurements of Effective Graphite Absorption," ORNL/M-2155 (June 1992). (4.8)

DIFILIPPO, F. C., AND R. B. PEREZ*

"Application of a New Integral Representation of the $K_{0}$ Bessel Function," Appl. Math. Lett. 5(5), 51 (1992). (4.12)

DONGARRA, J. J., S. HAMMARLING,* AND J. H. WILKINSON*

"Numerical Considerations in Computing Invariant Subspaces," SIAM J. Matrix Anal. Appl. 13(1), 145 (1992). (1.27)

DONGARRA, J. J., AND B. ROSENER*

"NA-NET Numerical Analysis Net," ORNL/TM-11986 (December 1991). (1.29)

DONGARRA, J. J., AND M. SIDANI*

"A Parallel Algorithm for the Non-Symmetric Eigenvalue Problem," ORNL/TM-12003 (December 1991). (1.30)

DONGARRA, J. J., AND R. A. VAN DE GEIJN*

"Reduction to Condensed Form for the Eigenvalue Problem on Distributed Memory Architectures," ORNL/TM-12006 (January 1992). (1.31)

DONGARRA, J. J., R. A. VAN DE GEIJN,* AND D. W. WALKER "A Look at Scalable Dense Linear Algebra Libraries," ORNL/TM-12126 (July 1992). (1.32)

DUNIGAN, T. H.

"Performance of the Intel iPSC/860 and Ncube 6400 Hypercubes," Parallel

Computing 17, 1285 (1991); also ORNL/TM-11790 (April 1991). (1.9) 
"Communication Performance of the Intel Touchstone Delta Mesh," ORNL/TM-11983 (January 1992). (1.6)

"Kendall Square Multiprocessor: Early Experiences and Performance," ORNL/TM-12065 (April 1992). (1.8)

"Hypercube Clock Synchronization," Concurrency: Practice and Experience 4(3), 257 (1992). (1.7)

EINSTEIN, J. R., R. J. MURAL,* X. GUAN, AND E. C. UBERBACHER

"Computer-Based Construction of Gene Models Using the GRAIL Gene Assembly Program," ORNL/TM-12174 (September 1992). (3.55)

EINSTEIN, J. R., E. C. UBERBACHER, X. GUAN, R. J. MURAL,* AND R. C. MANN

"GAP - A Computer Program for Gene Assembly," ORNL/TM-11924 (September 1991). (3.53)

FLANAGAN, D. M.

"Software Considerations for Reliability Centered Maintenance Analyses," ORNL/M-1530 (July 1991). (1.93)

FU, C. Y.

"Pairing Corrections and Spin Cutoff Factors in Exciton Level Densities for Two Kinds of Fermions," Nucl. Sci. Eng. 109, 18 (1991). (1991)

FU, C. Y., R. G. ALSMILLER, JR., AND T. A. GABRIEL "Liquid Argon Calorimeter Design Study Using the CALOR89 Code System," Nucl. Instrum. Methods Phys. Res. A311, 179 (1992). (4.30)

FUJIMURA, K.

"Motion Planning in Dynamic Environments," book published by SpringerVerlag, Tokyo (December 1991). (3.18)

\section{GAFF, S. J.,* AND V. PROTOPOPESCU} "Sustainability and Profitability in Ecological Systems with Harvesting," ORNL/TM-12151 (August 1992). (4.74)

\section{GEIST, G. A., AND V. S. SUNDERAM*}

"Network-Based Concurrent Computing on the PVM System," Concurrency: Practice and Experience 4(4), 293 (1992); also ORNL/TM-11760 (June 1991). (1.13) 
GILBERT, J. R.,* AND E. G. NG

"Predicting Structure in Nonsymmetric Sparse Matrix Factorizations," ORNL/TM-12204 (October 1992). (1.39)

\section{GILBERT, J. R.,* E. G. NG, AND B. W. PEYTON}

"An Efficient Algorithm to Compute Row and Column Counts for Sparse Cholesky Factorization," ORNL/TM-12195 (September 1992). (1.40)

GOSHAW, A. T.,* .. T. A. GABRIEL, G. T. ALLEY, C. L. BRITTON, JR., C. W. GLOVER, D. VANDERGRIFF,* H. R. BRASHEAR,* M. EMERY,* T. RYAN, et al.

"Hybrid Central Tracking Chamber Collaboration - Progress Report for FY 1991," ORNL/M-1775 (September 1991). (4.34)

GRAY, L. J.

"Boundary Element Analysis at Oak Ridge National Laboratory," International Association for Boundary Element Methods 5, 3 (1991). (1.50)

"Hypersingular Integral Evaluation Using Maple," Maple Newsletter 8, 57 (1992). (1.49)

GRAY, L. J., AND S. J. CHANG*

"Hypersingular Integral Formulation of Elastic Wave Scattering," Engineering Analysis with Boundary Elements 10, 337 (1992). (1.51)

GRAY, L. J., G. E. GILES, AND M. W. WENDEL*

"Boundary Element Method for Regions with Thin Internal Cavities. II," J. Engineering Analysis with Boundary Elements 8, 81 (1991). (1989)

HAALAND, C. M.

"Perturbed Environment Assessments for Space Vehicles," ORNL/TM-11722 (June 1991). (4.45)

"Minimum Engine Size for Optimum Automobile Acceleration," Am. J. Phys. 60(5), 415 (1992). (4.84)

HARBER, K. S.

"Proceedings of the Sixth International Symposium on Methodologies for Intelligent Systems," ORNL/TM-11938 (September 1991). (3.3)

\section{HEATH, M. T., AND J. A. ETHERIDGE}

"Visualizing the Performance of Parallel Programs," IEEE Software p. 29 (September 1991); also ORNL/TM-11813 (May 1991). (1.14) 
HORWEDEL, J. E.*

"GRESS Version 2.0 User's Manual," ORNL/TM-11951 (November 1991). (4.79)

HORWEDEL, J. E.,* R. J. RARIDON,* AND R. Q. WRIGHT*

"Automated Sensitivity Analysis of an Atmospheric Dispersion Model," Atmospheric Environment 26A(9), 1643 (1992). (4.82)

HU, P. S., * T. WRIGHT, S.-P. MIAOU,* R. GORMAN,* S. C. DAVIS* "A Study of Interstate Motor Carrier Vehicle Miles of Travel," Transpn. Res.. A 25A(6), 451 (1991). (1.97)

HUNT, G.,* AND Y. Y. AZMY

"Graphics Development of DCOR: Deterministic Combat Model of Oak Ridge," ORNL/TM-11973 (October 1992). (4.75)

INGERSOLL, D. T., AND J. K. INGERSOLL*

"Early Test Facilities and Analytic Methods for Radiation Shielding," ORNL/RSIC-55 (November 1992). (4.16)

IYENGAR, S. S.,* A. SABHARWAL,* F. G. PIN, AND C. R. WEISBIN*

"Asynchronous Production System for Control of an Autonomous Mobile Robot in Real Time Environment," Applied Artificial Intelligence 6, 485 (1992). (1991)

JESSUP, E. R.

"A Case Against a Divide and Conquer Approach to the Nonsymmetric Eigenvalue Problem," ORNL/TM-11903 (December 1991). (1.34)

JOB, P. K., ${ }^{*}$ L. E. PRICE,* J. PROUDFOOT, ${ }^{*}$ T. HANDLER,* AND T. A. GABRIEL

"Design Considerations for a Scintillating Plate Calorimeter," Nucl. Instrum. Methods Phys. Res. A316, 174 (1992). (4.37)

JOB, P. K., * L. E. PRICE,* J. PROUDFOOT,* T. HANDLER,* B. L. BISHOP,* AND T. A. GABRIEL

"Comparison of CALOR89 Model Predictions with Scintillator Plate Calorimeter Data," Nucl. Instrum. Methods Phys. Res. A309, 60 (1991). (4.36)

JOHNSON, J. O.

"A User's Manual for MASH 1.0 - A Monte Carlo Adjoint Shielding Code," ORNL/TM-11778 (March 1992). (4.70) 
JOHNSON, J. O., J. D. DRISCHLER, AND J. M. BARNES*

"Analysis of the Fall-1989 Two-Meter Box Test Bed Experiments Performed at the Army Pulse Radiation Facility (APRF)," ORNL/TM-11777 (May 1991). (4.48)

"Analysis of the Spring-1990 Two-Meter Box Test Bed Experiments Performed at the Army Pulse Radiation Facility (A:'RF)," ORNL/TM-11917 (August 1992). (4.49)

"Analysis of the Spring-1991 Two-Meter Box Test Bed Experiments Performed at the Army Pulse Radiation Facility (APRF)," ORNL/TM-11918 (September 1992). (4.50)

JONES, J. P., P. L. BUTLER,* S. E. JOHNSTON,* AND T. E. HEYWOOD*

"Hetero Helix: Synchronous and Asynchronous Control Systems in Heterogeneous Distributed Networks," Robotics and Autonomous Systems 10(2-3), 85 (1992). (3.8)

JONES, T. D.,* M. D. MORRIS, AND R. W. YOUNG*

"A Mathematical Model for Radiation-Induced Myelopoiesis," Radiation Research 128, 258 (1991). (1.79)

KAHANER, D. K.,* E. NG, W. E. SCHIESSER,* AND S. THOMPSON*

"Experiments with an Ordinary Differential Equation Solver in the Parallel Solution of Method of Lines Problems on a Shared Memory Parallel Computer," book chapter in Recent Advances in Numerical Methods and Software for ODEs/DAEs/DAEs/PDEs, pp. 7-36, World Scientific Publishing Company, 1992; also Journal of Computational and Applied Mathematics 38, 231 (1991). (1991)

KIRK, B. L., AND Y. Y. AZMY

"An Iterative Algorithm for Solving the Multidimensional Neutron Diffusion Nodal Method Equations on Parallel Computers," Nucl. Sci. Eng. 111, 57 (1992). (1991)

KLEIN, J. A.,* J. E. MROCHEK,* R. L. JOLLEY,* I. W. OSBORNELEE,* A. A. FRANCIS,* AND T. WRIGHT

"National Profile on Commercially Generated Low-Level Radioactive Mixed Waste," ORNL-6731 (December 1992). (1.98)

KRANER, H. W.,* .., G. T. ALLEY,* M. L. BAUER, ${ }^{*}$ C. L. BRITTON, JR., * H. R. BRASHEAR,* H. O. COHN, * T. A. GABRIEL, F. PLASIL, ${ }^{*}$ M. J. RENNICH, ${ }^{*}$ R. A. TODD,* et al.

"Silicon Electromagnetic Calorimeter Collaboration Progress Report," ORNL/M-1776 (September 1991). (4.39) 
LEAL, L. C.,* G. DE SAUSSURE, AND R. B. PEREZ*

"An R Matrix Analysis of the ${ }^{235} \mathrm{U}$ Neutron-Induced Cross Sections up to $500 \mathrm{eV}$," Nucl. Sci. Eng. 109, 1 (1991). (1991)

LENHART, S. M., S. STOJANOVIC,* AND V. PROTOPOPESCU

"Variational Approach for Competitive Systems with Obstacles," Appl. Math. and Computation 42, 65 (1991). (1991)

LEUZE, M. R., AND R. C. WARD

"Parallel Computers Take Lead in Complex Problem Solving," Computers in Physics 5(6), 582 (1991). (1.15)

LIEPINS, G. E., AND H. S. VACCARO*

"Intrusion Detection: Its Role and Validation," Computers \& Security 11, 347 (1992).

LIEPINS, G. E., AND M. D. VOSE*

"Polynomials, Basis Sets, and Deceptiveness in Genetic Algorithms," Complex Systems 5, 45 (1991). (3.39)

MAIENSCHEIN, F. C., AND R. W. PEELLE

"Letter to the Editor of the Journal of the American Medical Association on Mortality Among Workers at Oak Ridge National Laboratory by S. Wing et al.," JAMA 267(7), 929 (1992).

MANN, R. C., M. BECKERMAN, K. FUJIMURA, J. P. JONES, E. M. OBLOW, F. G. PIN, AND M. A. UNSEREN

"Proposal for Continued Basic Research in Intelligent Machines at the Center for Engineering Systems Advanced Research Fiscal Years 1992 - 1996," ORNL/TM-11810 (May 1991).

MARTHA, L. F., * A. R. INGRAFFEA,* AND L. J. GRAY

"Three-Dimensional Fracture Simulation with a Single-Domain, Direct Boundary Element Formulation," Int. J. for Num. Methods in Engineering 35, 1907 (1992). (1.59)

MIAOU, S.-P.,* P. S. HU,* T. WRIGHT, A. K. RATHI,* AND S. C. DAVIS*

"Relationship Between Truck Accidents and Highway Geometric Design: A Poisson Regression Approach," Transportation Research Record 1376, 10 (1992). (1.104) 
MILLER, M. A.,* AND J. K. DICKENS

"Study of Radionuclides Created by ${ }^{181} \mathrm{Ta}(\gamma, x n y p)$ Reactions for Bremsstrahlung Photons Produced by $150-\mathrm{MeV}$ Electrons," ORNL/TM11944 (December 1991). (2.8)

MITCHELL, T. J.

"Formed Derivations of the Disappearance of the High Survival as a Function of Temperature and Cooling Rate," (appendix) Cryobiology 29, 39 (1992).

MITCHELL, T. J., AND M. D. MORRIS

"Bayesian Design and Analysis of Computer Experiments: Two Examples," Statistica Sinica 2, 359 (1992). (1.80)

MORRIS, M. D.

"On Counting the Number of Data Pairs for Semivariogram Estimation," Mathematical Geology 23(7), 929 (1991). (1991)

MORRIS, M. D., AND T. D. JONES*

"Marrow Cell Kinetics Model: Equivalent Prompt Dose Approximations for Two Special Cases," ORNL/TM-12255 (November 1992). (1.105)

MORRIS, M. D., T. D. JONES,* AND R. W. YOUNG

"Estimation of Coefficients in a Model of Radiation-Induced Myelopoiesis from Mortality Data for Mice Following X-Ray Exposure," Radiation Research 128, 267 (1991). (1991)

MORRIS, M. D., AND T. J. MITCHELL

"Exploratory Designs for Computational Experiments," ORNL/TM12045 (October 1992). (1.83)

MORRIS, M. D., T. J. MITCHELL, AND D. YLVISAKER*

"Bayesian Design and Analysis of Computer Experiments: Use of Derivatives in Surface Prediction," ORNL/TM-11699 (June 1991). (1.84)

MURAL, R. J., J. R. EINSTEIN, X. GUAN, R. C. MANN, AND E. C. UBERBACHER

"An Artificial Intelligence Approach to DNA Sequence Feature Recognition," Trends in Biotechnology 10, 66 (1992). (3.52)

NANSTAD, R. K., ${ }^{*}$ F. M. HAGGAG,* D. E. MCCABE, ${ }^{*}$ S. K. ISKANDER, ${ }^{*}$ K. O. BOWMAN, AND B. H. MENKE*

"Irradiation Effects on Fracture Toughness of Two High-Copper SubmergedArc Welds, HSSI Series 5," ORNL/TM-12156 (September 1992). 
NEVES, K. W.,* AND S. THOMPSON*

"Software for the Numerical Solution of Systems of Functional Differential Equations with State Dependent Delays," Applied Numerical Mathematics 9, 385 (1992). (1991)

NG, E.

"Supernodal Symbolic Cholesky Factorization of a Local-Memory Multiprocessor," ORNL/TM-11836 (June 1991). (1.41)

NG, E., AND B. W. PEYTON

"A Supernodal Cholesky Factorization Algorithm for Shared-Memory Multiprocessors," ORNL/TM-11814 (April 1991). (1.42)

"Block Sparse Cholesky Algorithms on Advanced Uniprocessor Computers," ORNL/TM-11960 (December 1991). (1.44)

"A Tight and Explicit Representation of Q in Sparse QR Factorization," ORNL/TM-12059 (May 1992). (1.43)

OBLOW, E. M.

"Implementing Valiant's Learnability Theory Using Random Sets," Machine Learning 8, 45 (1992). (3.26)

OBLOW, E. M., AND V. R. R. UPPULURI

"Analysis of Complexity Bounds for Pac-Learning with Random Sets," ORNL/TM-11844 (August 1991). (3.29)

OSTROUCHOV, G.

"Computer Communication: What's Your E-Mail Address?" Statistical Computing and Statistical Graphics Newsletter 2(2), 22 (1991). (1.107)

"Computer Communication: Resource Discovery," Statistical Computing and Statistical Graphics Newsletter 3(1), 21 (1992). (1.108)

"Computer Communication: Alternate Connections and White Pages," Statistical Computing and Statistical Graphics Newsletter 3(2), 22 (1992). (1.109)

PEYTON, B. W., A. POTHEN,* AND X. YUAN*

"Partitioning a Chordal Graph Into Transitive Subgraphs for Parallel Sparse Triangular Solution," ORNL/TM-12270 (December 1992). (1.45)

PIN, F. G.

"Robotics and Intelligent Systems Research at the Oak Ridge National Laboratory," Robotics and Autonomous Systems 10(2), 81 (1992). (3.2) 
PIN, F. G., AND J.-C. CULIOLI*

"Optirnal Positioning of Combined Mobile Platform-Manipulator Systems for Material Handling Tasks," Journal of Intelligent and Robotic Systems 6, 165 (1992). (1991)

PIN, F. G., L. E. PARKER,* AND F. W. DEPIERO*

"On the Design and Development of a Human-Robot Synergistic System," Robotics and Autonomous Systems 10(2), 161 (1992). (3.50)

PRIMM, R. T., III

"Reactor Physics Input to the Safety Analysis Report for the High Flux Isotope Reactor," ORNL/TM-11956 (March 1992). (4.19)

PRIMM, R. T., III, C. M. HOPPER,* AND G. R. SMOLEN* "Criticality Safety Studies for the Storage of Waste from Nuclear Fuel Services in Intercell Storage Wells 2 and 3 of Building 3019," ORNL/TM-12152 (November 1992). (4.21)

PROTOPOPESCU, V.

"Integral Methods for Solving Fokker-Planck-Type Equations," Integral Methods in Science and Engineering-90, A. Haji-Sheikh, C. Corduneanu, J. L. Fry, T. Huang, and F. R. Payne, Editors, p. 111 (1991). (1991)

PROTOPOPESCU, V., AND Y. Y. AZMY

"Topological Chaos for a Class of Linear Models," Mathematical Methods and Models in Applied Sciences 2, 79 (1992). (4.76)

PROTOPOPESCU, V., R. YAGER,* AND J. DOCKERY*

"Combat Modeling with Imprecise Data," Int. J. of Intelligent Systems 7, 277 (1992). (4.78)

RAMAN, S., * B. L. BROADHEAD,* J. K. DICKENS, R. L. WALKER,* AND J. L. BOTTS*

"Analyses of Physics Specimens in Fuel Pins 1 and 2 Irradiated in the Dounreay Prototype Fast Reactor," ORNL-6632 (January 1992). (2.44)

RAO, N. S. V.,* E. M. OBLOW, AND C. W. GLOVER

"N-Learners Problem: Learning Boolean Combinations of Halfspaces," ORNL/TM-11905 (March 1992). (3.27)

RAO, N. S. V., ${ }^{*}$ E. M. OBLOW, C. W. GLOVER, AND G. E. LIEPINS "N-Learners Problem: Fusion of Concepts," ORNL/TM-11904 (September 1991). (3.28) 
RAO, N. S. V.,* W. WU,* AND C. W. GLOVER

"Algorithms for Recognizing Planar Polygonal Configurations Using Perspective Images," IEEE Transactions on Robotics and Automation 8(4), 480 (1992). (3.30)

REISTER, D. B.

"A New Wheel Control System for the Omnidirectional HERMIES-III Robot," Robotica 10(4), 351 (1992); also ORNL/TM-11784 (April 1991). (3.5)

REISTER, D. B., AND A. S. GUTH*

"The Bang-Bang Production of Depletable Natural Resources," International Review of Economics and Business 39, 5 (1992).

REISTER, D. B., AND F. G. PIN

"Time Optimal Trajectories for Mobile Robots with Two IndependentlyDriven Wheels," ORNL/TM-12070 (March 1992). (3.6)

\section{REISTER, D. B., AND M. A. UNSEREN}

"Position and Force Control of a Vehicle with Two or More Steerable Drive Wheels," ORNL/TM-12193 (October 1992).

\section{RHOADES, W. A., R. L. CHILDS,* AND D. T. INGERSOLL}

"Radiation Exposure Inside Reinforced Concrete Buildings at Nagasaki," Health Physics 63(5), 510 (1992). (4.53)

\section{RHOADES, W. A., R. A. LILLIE, AND M. B. EMMETT*}

"Transmission Factors for the Penetration of Neutron and Photon Fluence into Wood-Frame Dwellings, 1990 (TF90)," ORNL/TM-12021 (May 1992). (4.54)

RICE, A. F., AND R. W. ROUSSIN (Editors)

"Deterministic Methods in Radiation Transport," ORNL/RSIC-54 (June 1992). (5.11)

SANTORO, R. T.

"DNA Radiation Environments Program Fall 1989 2-Meter Box Experiments and Analysis," ORNL/TM-11840 (May 1991). (4.55)

SANTORO, R. T., J. D. DRISCHLER, AND J. O. JOHNSON

"Resolution of Dose and Reduction Factor Questions in Army Pulse Radiation Facility Experiments: Renormalization of Calculated and Measured Data," ORNL/TM-12148 (November 1992). (4.56) 
SANTORO, R. T., AND D. T. INGERSOLL

"Radiation Shielding Requirements for Manned Deep Space Missions," ORNL/TM-11808 (April 1991). (4.40)

SANTORO, R. T., AND S. Y. WHITAKER*

"DNA Radiation Environments Program Spring 1990 2-Meter Box Experiments and Analyses," ORNL/TM-12160 (September 1992). (4.57)

SCHMOYER, R. L.

"Asymptotically Valid Prediction Intervals for Linear Models," Technometrics 34(4), 399 (1992). (1.91)

SCHRYVER, J. C.

"Object-Oriented Qualitative Simulation of Human Mental Models of Complex Systems," IEEE Transactions on Systems, Man, and Cybernetics 22(3), 526 (1992). (1991)

SLATER, C. $O$.

"DRC2: A Code with Specialized Applications for Coupling Localized Monte Carlo Adjoint Calculations with Fluences from Two-Dimensional R-Z Discrete Ordinates Air-Over-Ground Calculations," ORNL/TM-11873 (January 1992). (4.71)

SOLOMON, A. D., J. E. NYQUIST,* V. ALEXIADES, G. K. JACOBS,* AND S. M. LENHART

"A Preliminary Study of the Controls on Melting During In Situ Vitrification," ORNL/ER-30 (December 1991). (1.62)

SPELT, P. F.

"Survey of Human Factors Applications of Artificial Neural Networks," Human Factors Society Bulletin 35(3), 4 (1992). (3.44)

"Introduction to Artificial Neural Networks for Human Factors," Human Factors Society Bulletin 34(7), 1 (1991). (3.45)

SPELT, P. F., KNEE, H. E., AND C. W. GLOVER

"Hybrid Artificial Intelligence Architecture for Diagnosis and Decision-Making in Manufacturing," Journal of Intelligent Manufacturing 2, 261 (1991). (1991)

STEINER, R.,* ... R. G. ALSMILLER, JR., C. Y. FU, T. A. GABRIEL, et al.

"Liquid Argon Calorimetry for the SSC Progress Report for FY 91 and Request for Funds for FY 92," ORNL/M-1773 (September 1991). (4.41) 
STORJOHANN, K.,* AND E. SALTZEN*

"Laser Range Camera Calibration," ORNL/TM-11723 (July 1991). (3.34)

TRUBEY, D. K.

"JANE: A New Information Retrieval System for the Radiation Shielding Information Center," ORNL/RSIC-53 (May 1991). (5.10)

"New Gamma-Ray Buildup Factor Data for Point Kernel Calculations: ANS6.4.3. Standard Reference Data," ORNL/RSIC-49/R1 (August 1991). (5.3)

\section{UNSEREN, M. A.}

"Rigid Body Dynamics and Deccupled Control Architecture for Two Strongly Interacting Manipulators," Robntica 9, 421 (1991). (1991)

"A Rigid Body Model and Decoupled Control Architecture for Two Manipulators Holding a Complex Object," Robotics and Autonomous Systems 10(2), 115 (1992). (1991)

"Input Relegation Control for Gross Motion of a Kinematically Redundant Manipulator," ORNL/TM-12165 (October 1992). (3.11)

VANDERGRIFF, D. H.*

"Preliminary Engineering Studies for the Support Shell of the Outer Tracker of the SDC Detector," ORNL/TM-11937 (September 1991).

VASSEUR, H. A.,* F. G. PIN, AND J. R. TAYLOR*

"Navigation of Car-Like Mobile Robots in Obstructed Environments Using Convex Polygonal Cells," Robotics and Autonomous Systems 10(2), 133 (1992). (1591)

VINEYAR'), T. A.,* D. J. DOWNING, R. C. DURFEE,* J. J. EDWARDS, * D. M. FLANAGAN, M. C. FLETCHER, ${ }^{*} \mathbf{R}$. T. GOELTZ,* G. OSTROUCHOV, J. A. ROME,* M. J. SALTMARSH,* J. L. SMYRE,* W. R. WING*

"Assessment of en Route Sector and Terminal Air Traffic Control Performance," K/DSRD-733 (June 1991). (1.112)

WALKER, i. W.

"A Fortran 50 Code for Magnetohydrodynamics, Part I: Banded Convolution," ORNL/TM-12032 (March 1992). (1.73)

"Particle-In-Cell Plasma Simulation Codes on the Connection Machine," Computing Systems in Engineering 2, 307 (1991). (1991)

"The Hierarcbical Spetial D composition of Three-Dimensional Particle-InCell Plasma Simulaiions o". MIMD Distributed Memory Multiprocessors," ORNL/TM-12071 (July 1 (J)). (1.72) 
"Standards for Message-Passing in a Distributed Memory Environment," ORNL/TM-12147 (August 1992). (1.19)

WALKER, D. W., P. H. WORLEY, AND J. B. DRAKE

"Parallelizing the Spectral Transform Method - Part II," Concurrency: Practice and Experience 4(7), 509 (1992); also ORNL/TM-11855 (July 1991). (1.76)

WELCH, W. J.,* R. J. BUCK, ${ }^{*}$ J. SACKS,* H. P. WYNN,* T. J. MITCHELL, AND M. D. MORRIS

"Screening, Predicting, and Computer Experiments," Technometrics 34(1), 15 (1992). (1991)

\section{WESTON, L. W., AND J. H. TODD*}

"Measurement of the ${ }^{10} \mathrm{~B}\left(n, \alpha_{0}\right) /{ }^{10} \mathrm{~B}(n, \alpha \gamma)$ Ratio Versus Neutron Energy," Nucl. Sci. Eng. 109, 113 (1991). (1991)

"High Resolution Fission Cross Section Measurements of ${ }^{235} \mathrm{U}$ and ${ }^{239} \mathrm{Pu}$," Nucl. Sci. Eng. 111, 415 (1992). 2.17)

WHITE, J. E., R. Q. WRIGHT,* R. W. ROUSSIN, AND D. T. INGERSOLL

"Specifications for the Development of Bugle-93: An ENDF/B-VI Multigroup Cross Section Library for LWR Shielding and Pressure Vessel Dosimetry," ORNL/TM-12230 (November 1992). (5.4)

WILliaMS, M. L., ${ }^{*}$ C. ABOUGHANTOUS, ${ }^{*}$ M. ASGARI,* J. E. WHITE, R. Q. WRIGHT,* AND F. R Kr. KAM*

"Transport Calculations of Neutron Transmission Through Steel Using ENDF/B-V, Revised ENDF/B-V and ENDF/B-VI Iron Evaluations," Ann. Nucl. Energy 18(10), 549 (1991). (5.6)

WILLIAMSON, D. L., * J. B. DRAKE, J. J. HACK, ${ }^{*}$ R. JAKOB,* AND P. N. SWARZTRAUBER*

"A Standard Test Set for Numerical Approximations to the Shallow Water Equations in Spherical Geometry," ORNL/TM-11895 (August 1991). (1.77)

WORLFY, P. H.

"Parallelizing Across Time When Solving Time-Dependent Partial Differential Equations," ORNL/TM-11963 (September 1991). (1.36)

"A New PICL Trace File Format," ORNL/TM-12125 (October 1992). (1.20)

"The Effect of Multiprocessor Radius on Scaling," Parallel Computing 18(1), 361 (1992). (1.23) 
WORLEY, P. H., AND J. B. DRAKE

"Parallelizing the Spectral Transform Method," Concurrency: Practice and Experience 4(4), 269 (1992). (1.78)

WRIGHT, T.

"Lagrange's Identify Reveals Correlation Coefficient and Straight-Line Connection," The American Statistician 46(2), 106 (1992). (1991)

"A Note on Sampling to Locate Rare Defectives with Strong Prior Evidence," Biometrika 79(4), 685 (1992). (1.99)

ZEITNITZ, C.,* G. LINDSTRÖM,* E. FRETWURST,* V. RIECH,* T. A. GABRIEL, AND T. HANDLER*

"Simulation of a Presampler Response with CALOR - A Comparison with Experimental Data," DESY 92-045 (March 1992). (4.42)

ZHAO, Z., ${ }^{*}$ AND F. G. PEREY

"The Covariance Matrix of Derived Quantities and Their Combination," ORNL/TM-12106 (June 1992). (2.39)

\section{ZHIXIANG, Z.,* C. Y. FU, AND D. C. LARSON}

"Theoretical Calculation of Neutron Induced Data of ${ }^{19} \mathrm{~F}$ and Uncertainties of Parameters," Chinese Journal of Nuclear Physics 14(1), 67 (1992). (2.27) 


\section{PAPERS PRESENTED AT SCIENTIFIC MEETINGS AND SEMINARS}

ANS Topical Meeting on Advances in Human Factors Research on Man-Computer Ineractions: Nuclear and Beyond, Nashville, TN, June 10-14, 1990; Proc. (1990)

SAWHNEY, R. S., * J. C. SCHRYVER, AND H. L. DODDS,* "An Operator Model-Based Filtering Scheme," p. 111.

International Atomic Energy Agency Final Meeting of a Co-ordinated Research Programme, Vienna, Austria, June 20-22, 1990; Proc. (1991)

YOUNG, P. G., ${ }^{*}$ R. C. HAIGHT, ${ }^{*}$ R. O. NELSON, ${ }^{*}$ S. A. WENDER, ${ }^{*}$ C. M. LAYMON,${ }^{*}$ G. L. MORGAN, ${ }^{*}$ D. M. DRAKE,${ }^{*}$ M. DROSG,${ }^{*}$ H. VONACH, ${ }^{*}$ A. PAVLIK, ${ }^{*}$ S. TAGESEN, ${ }^{*}$ D. C. LARSON, AND D. S DALE,* "Calculation of $(\mathrm{n}, \mathrm{x} \gamma)$ Cross Sections Between Threshold and $100 \mathrm{MeV}$ for $\mathrm{Fe}$ and $\mathrm{Pb}$ Isotopes: Comparison with Experimental Data," pp. 239-250. $(\mathbf{2 . 2 6})^{\dagger}$

IABEM Symposium, Rome, Italy, October 15-19, 1990; Proc. Boundary Integral Methods, Springer Verlag (1991)

LUTZ, E., L. J. GRAY, AND A. R. INGRAFFEA," "Indirect Evaluation of Surface Stress in the Boundary Element Method," pp. 339-348. (1.57)

IMACS International Symposium on Iterative Methods in Linecir Algebra, Brussels, Belgium, April 2-5, 1991; Proc. (1991)

FORSYTH, P. A., * W. P. TANG, * AND E. F. D'AZEVEDO, "Two Variants of Minimum Discarded Fill Ordering," pp. 603-612. (1.70)

1991 IEEE International Conference on Robotics and Automation, Sacramento, CA, April 7-12, 1991; Proc. (1991)

FUJIMURA, K., "A Model of Reactive Planning for Multiple Mobile Agents,"

Vol. 2, pp. 1503-1509. (3.17)

REISTER, D. B., "A New Wheel Control System for the Omnidirectional HERMIES-III Robot," Vol. 3, pp. 2322-2327. (3.5)

REISTER, D. B., J. P. JONES, P. L. BUTLER, ${ }^{*}$ M. BECKERMAN, AND F. J. SWEENEY, "DEMO 89 - The Initial Experiment with the HERMIESIII Robot," Vol. 3, pp. 2562-2567. (3.14)

VASSEUR, H. A., F. G. PIN, AND J. R. TAYLOR,* "Navigation of a CarLike Mobile Robot Using a Decomposition of the Environment in Convex Cells," Vol. 3, pp. 1496-1502. (3.20)

* Not a member of Engineering Physics and Mathematics Division.

$\uparrow$ The number shown in parentheses following the publication corresponds to the number of an abstract included in this report. If a number does not appear, then no abstract was available for this report. 
Workshop on Detector and Event Simulation in High Energy Physics, Amsterdam, Netherlands, April 8-12, 1991

JOB, P. K., ${ }^{*}$ J. PROUDFOOT,* T. HANDLER, * AND T. A. GABRIEL, "Systematic Effects in CALOR Simulation Code to Model Experimental Configurations." (4.38)

Oral Presentation, Fifteenth Annual Meeting of the Southeastern Atlantic Section of SIAM, Cullowhee, NC, April 12, 1991

DRAKE, J. B., "Parallel Computation of Atmospheric Flows."

Workshop on Variational Problems, Institute for Mathematics and Its Applications, Minneapolis, MN, April 15-19, 1991; Proc. (1999)

LENHART, S., V. PROTOPOPESCU, AND S. STOJANOVIC,* "A Two-

Sided Game for Non Local Competitive Systems with Control on Source Terms." (1.55)

Oral Presentation, Conference on High Speed Computing, Salishan Lodge, OR, April $16-18,1991$

GEIST, G. A., "Parallel Superconductor Code Exceeds 2.5 Gflops on iPSC/860."

American Physical Society Spring Meeting, Washington, DC, April 21-25, 1991;

Proc. Vol. 96(4) (1991)

CARLTON, R. F., * R. R. WINTERS,* J. A. HARVEY, N. W. HILL,* C. H. JOHNSON,* AND J. SCHMIEDMAYER,* "R-Matrix Analysis of an ORELA Measurement of the $n+{ }^{208} \mathrm{~Pb}$ Total Cross Section from 78 to 1700 $\mathrm{keV}, "$ p. 1349. (2.19)

Computing Science and Statistics: Interface 1991, Seattle, WA, April 22-24, 1991; Proc. (1991)

MITCHELI, T. J., AND M. D. MORRIS, "Using Computer Experiments to Construct a Cheap Substitute for an Expensive Simulation Model," pp. 272-277. (1.81)

IBM Large Scale Computer Analysis and Modeling Conference, Yorktown, NY, April 24-26, 1991

SUNDERAM, V. S., * AND G. A. GEIST, "The PVM System: Supercomputer Level Concurrent Computation on a Network of IBM RS/6000 Powerstations." (1.18)

Oral Presentation, Parallel Eigensolvers Workshop, Richland, WA, April 25-26, 1991

JESSUP, E. R., "A Divide and Conquer Method for the Nonsymmetric Eigenvalue Problem." 
ANS Topical Meeting on Advances in Mathematics, Computation, and Reactor Physics, Pittsburgh, PA, April 28-May 1, 1991; Proc. (1991)

AZMY, Y. Y., "A Comparison Between the Finite Difference and Nodal Integral Methods for the Neutron Diffusion Equation," Vol. 4, p. 16.1, 21. (4.58)

DELEANU, L., * Y. Y. AZMY, AND R. T. PRIMM, III, "The WeightedDifference Form of the Nodal Diffusion Method in Cylindrical Geometry," p. 16.1, 3-1. (4.67)

DIFILIPPO, F. C., AND K. MACKO,* "Stochastic Processes in Nuclear Reactors Under Parametric Excitation," Vol. 1, p. 5.2, 1-1. (4.11)

HAGHIGHAT, A.,* AND Y. Y. AZMY, "Parallelization of a Spherical $S_{N}$ Algorithm Based on the Spatial Domain Decomposition," Vol. 1, p. 1.1. (4.69)

RHOADES, W. A., AND R. L. CHILDS, * "TORT-Three Dimensional Oak Ridge Discrete Ordinate Neutron/Photon Transport Code," Vol. 5, p. 30.3, $1-1$.

6th Distributed Memory Computing Conference, Portland, OR, April 28-May 2, 1991; Proc. (1991)

GEIST, G. A., AND V. S. SUNDERAM,* "The PVM System: Supercomputer Level Concurrent Computation on a Heterogeneous Network of Workstations," pp. 258-261. (1.12)

GUAN, X., R. C. MANN, R. MURAL, * AND E. UBERBACHER, "Searching for Consensus Patterns on a Hypercube," pp. 470-472. (3.57)

WORLEY, P. H., J. B. DRAKE, AND D. W. WALKER, "Parallelizing the Spectral Transform Method," pp. 306-313. (1.78)

Oral Presentation, Sixth Distributed Memory Computing Conference, Portland, OR, April 29, 1991

CRIVELLI, S.," AND E. R. JESSUP, "Toward an Efficient Parallel Implementation of the Bisection Method."

Workshop on Undergraduate and Graduate Education, Louisiana State University, Baton Rouge, LA, April 29-90, 1991; Proc. (1991)

GABRIEL, T. A., "The Computational Challenges Provided by the Codes Used to Design and Analyze Superconducting Super Collider Detectors," pp. 105-117. (4.31)

22nd International Conference on Modeling and Simulation, Pittsburgh, PA, May 2-3, 1991; Proc. Vol. 5 (1991)

PROTOPOPESCU, V., R. T. SANTORO, Y. Y. AZMY, AND B. L. KIRK, "Analytic Modeling vs Simulation for Classical Warfare," p. 2480. (4.77) 
Operations Research Society of America Confer'nce, Nashville, TN, May 5-8, 1991 CARTER, R. J., "A Training Effectiveness Program for Training Systems." (3.49)

Oral Presentation, Superconducting Super Collider Laboratory, Dallas, TX, May 7, 1991

GABRIEL, T. A., "SSC Detector Simulation and Development."

Oral Presentation, Department of Mathematical Sciences Colloquium, Clemson University, Clemson, SC, May 10, 1991

PEYTON, B. W., "Parallel Sparse Cholesky Algorithms."

The Institute of Management Science/Operations Research Society of America (TIMS/ORSA) Joint National Meeting, Nashville, TN, May 12-15, 1991; Proc. Vol. 31 (1991)

HORWEDEL, J. E.,* "GRESSing Along," p. 63. (4.80)

PROTOPOPESCU, V., R. T. SANTORO, Y. Y. AZMY, AND B. L. KIRK, "Modeling Conflict Using Partial Differential Equations," (oral presentation only).

SANTORO, R. T., V. PROTOPOPESCU, G. W. HUNT, ${ }^{*}$ Y. Y. AZMY, AND B. L. KIRK, "PDE Models: Comparison with Other Simulation Method Data," (oral presentation only).

Ninth Symposium on Energy Engineering Sciences, Argonne National Laboratory, Argonne, IL, May 13-15, 1991; Proc. (1991)

PIN, F. G., AND R. S. PATTAY,* "Control of Autonomous Mobile Robots Using C'stom-Designed Qualitative Reasoning VLSI Chips and Boards," pp. $141-14 \varepsilon,(3.21)$

Internationul Conference on Nuclear Data for Science and Technology, Julich, Federal Republic of Germany, May 19-17, 1991; Proc. S. M. Qaim, Ed., SpringerVerlag (1992)

DE SAUSSURE, G., AND N. M. LARSON,* "Multilevel Resonance Analysis of ${ }^{59}$ Co Transmission Measurements," pp. 109-111. (2.21)

DICKENS, J. K., C. Y. FU, D. M. HETRICK,* D. C. LARSON, AND J. H. TODD,* "Experimental and Calculated Excitation Functions for Discrete-Line Gamma-Ray Production Due to 1-40 MeV Neutron Interactions with ${ }^{56} \mathrm{Fe}$," pp. 307-309. (2.1)

DIFILIPPO, F. C., "Effects of the Two-Fission Neutron Distributions in Reactivity Measurements with the ${ }^{252}$ Cf Source," pp. 122-123. (4.5)

DIFILIPPO, F. C., "Cross Sections for Actinide Burner Reactors," pp. 95-97. (4.4) 
FU, C. Y., D. C. LARSON, D. M. HETRICK, ${ }^{*}$ H. K. VONACH, ${ }^{*}$ J. KOPECKY,* S. IIJIMA, ${ }^{*}$ N. YAMAMURO, ${ }^{*}$ AND G. MAINO,* "International Evaluation Cooperation Task 1.1: Intercomparison of Evaluated Files for ${ }^{52} \mathrm{Cr},{ }^{56} \mathrm{Fe}$, and ${ }^{58} \mathrm{Ni}$," pp. 857-859. (2.28)

HARVEY, J. A., N. W. HILL, ${ }^{*}$ AND N. M. LARSON, * "Measurement of the Nitrogen Total Cross Section from $0.5 \mathrm{eV}$ to $50 \mathrm{MeV}$, and Analysis of the 433-keV Resonance," pp. 729-731. (2.4)

HETRICK, D. M.,* D. C. LARSON, N. M. LARSON, ${ }^{*}$ C. Y. FU, AND S. J. EPPERSON, " "Evaluation of the Silicon Isotopes for ENDF/B-VI," pp. 909-911. (2.31)

LARSON, D. C., J. K. DICKENS, R. O. NELSON,* AND S. A. WENDER,* "White Source Gamma-Ray Production Spectral Measurement Facilities in the U.S.," pp. 422-426. (2.7)

PEELLE, R. W., "Progress and Problems in Energy-Related Nuclear Data," pp. 1011-1013. (2.9)

PEREY, C. M., F. G. PEREY, J. A. HARVEY, N. W. HILL, ${ }^{*}$ AND N. M. LARSON, ${ }^{*}{ }^{466} \mathrm{Fe}$ and ${ }^{60} \mathrm{Ni}$ Resonance Parameters," pp. 41-43. (2.11)

ROUSSIN, R. W., J. E. WHITE, E. SARTORI,* G. PANINI,* R. MACFARLANE,* D. MUIR,* M. MATTES,* I. HASEGAWA,* H. TAKANO, ${ }^{*}$ AND F. MANN,* "International Evaluation Cooperation Subgroup 7: Multigroup Cross Section Processing," pp. 860-862. (5.5)

SCHMIEDMAYER, J., ${ }^{*}$ P. RIEHS,* J. A. HARVEY, AND N. W. HILL,* "Electric Properties of the Neutron from Precision Cross Section Measurements," pp. 163-165. (2.12)

SCHRACK, R. A.,* O. A. WASSON, ${ }^{*}$ D. C. LARSON, J. K. DICKENS, AND J. H. TODD, ${ }^{*}$ "Measurement of the ${ }^{10} \mathrm{~B}(\mathrm{n}, \alpha \gamma)$ Cross Section in the 0.3to $4-\mathrm{MeV}$ Neutron Energy Interval," pp. 507-509. (2.14)

National Conference of American Society of Civil Engineers, Water Resource Planning Division on Water Resources Planning and Management, New Orleans, LA, May 20-22, 1991; Proc. Vol. 18 (1991)

HORWEDEL, J. E.,* AND S. F. RAILSBACK,* "An Automated Sensitivity Analysis Procedure Applied to a Dissolved Oxygen Simulation Model," pp. 484-488. (4.81)

Oral Presentation, Calorimetry Workshop for the Solenoidal Detector Collaboration, Chicago, IL, May 21-22, 1991

GABRIEL, T. A., R. G. ALSMILLER, JR., C. Y. FU, T. HANDLER,* J. PANAKKAL, ${ }^{*}$ J. PROUDFOOT, ${ }^{*}$ B. MOORE, ${ }^{*}$ AID J. REIDY,* "CALOR89 Calorimeter Simulations Benchmarking and Design Calculations: A Summary of Progress." 
Nuclear Energy Agency Nuclear Data Committee Specialists' Meeting on Neutron Cross Section Standards for the Energy Region Above $20 \mathrm{MeV}$, Uppsala, Sweden, May 21-29, 1991; Proc. NEANDC.905 "U" Organization for Economic Cooperation and Development, (1991)

DICKENS, J. K., "Scintillation Detector Efficiencies for Neutrons in the Energy Region Above $20 \mathrm{MeV}$," pp. 142-153. (2.42)

Oral Presentation, IBM Workstation Symposium Garden Plaza Hotel, Oak Ridge, TN, May 22, 1991

GEIST, G. A., "The PVM System: Supercomputer Level Concurrent Computation on a Heterogeneous Network of Workstations."

Oral Presentation, Shell Development Company, Houston, TX, May 90, 1991

GEIST, G. A., "The PVM System: Supercomputer Level Concurrent Computation on a Heterogeneous Network of Workstations."

1991 Summer Meeting of the American Nuclear Society, Orlando, FL, June 2-6, 1991; Trans. Am. Nucl. Soc. 63 (1991)

AZMY, Y. Y., "Acceleration of the Second Angular Moment in Larsen's DSA Formalism," p. 183.

CRAMER, S. N., AND R. D. DABBS,* "SARP Shielding Analysis at the Oak Ridge Y-12 Plant," p. 367.

DE SAUSSURE, G., N. M. LARSON,* AND J. A. HARVEY, "Evaluation of the ${ }^{59}$ Co Resolved Resonance Region," p. 425.

DRISCHLER, J. D., M. S. SMITH, D. H. WOOD, AND E. KOMORI,* "CREDO Status Report: 1990-1991," p. 280.

MANNESCHMIDT, J. F.," AND V. M. FORSBERG,* "CREDO in Transition: JOSHUA to Data Base 2," p. 279.

PEELLE, R. W., "Cross Section Research at the Oak Ridge Electron Linear Accelerator," p. 172.

PRIMM, R. T., III, "Fabrication Constraints Applied to the Reactor Physics Design of the Advanced Neutron Source," p. 443.

SLATER, C. O., J. V. PACE, III, R. L. CHILDS, ${ }^{*}$ M. J. HAIRE, ${ }^{*}$ AND T. KOYAMA, " "Three-Dimensional Radiation Dose Mapping with TORT Computer Code," p. 368.

WOOD, D. H., M. S. SMITH, J. D. DRISCHLER, AND E. KOMORI,* "Assessment of Early, Useful, and Wear-Out Operating Periods for LiquidMetal Centrifugal Pumps," p. 278. 
1991 IEEE International Conference on Plasma Science, Williamsburg, VA, June 9-5, 1991; Proc. (1991)

HAALAND, C. M., "Electric Power Generation by Steady Flow Liquid Metal MHD Generators," p. 200. (4.83)

Oral Presentation, PICS Organizational Meeting, Greenville, SC, June 6-7, 1991 GEIST, G. A., "HPCC Software Tools."

Institute for Mathematical Statistics Special Topics Meeting on Industrial Statistics, Philadelphia, PA, June 10-12, 1991; Proc. IMS Bulletin (1991)

BECKERMAN, M., "Sensor Data Fusion for Mobile Robots," Vol. 20, pp. 162-163. (3.36)

MORRIS, M. D., AND T. J. MITCHELL, "Exploratory Designs for Computational Experiments." (1.83)

Oral Presentation, PICS Material Science Workshop, Hilton Hotel, Alcoa, TN, June 17, 1991

GEIST, G. A., "HPCC Software Tools."

Third Topical Seminar on Heavy Flavours, San Miniato, Tuscany, Italy, June 1721, 1991

ANASSONTZIS, S., ${ }^{*}$ H. BRASHEAR, ${ }^{*}$ N. ERICISON, ${ }^{*}$ T. A. GABRIEL, T. MOORE,* AND A. WINTENBERG, et al., "The Super Fixed Target Beauty Facility at the SSC." (4.29)

Oral Presentation, IBM Europe Institute 1991, Oberlech, Austria, July 1-5, 1991 GEIST, G. A., "Supercomputer Performance Using a Network of RISC RS/6000's."

9th International Congress on Radiation Research, Toronto, Canada, July 7-12, 1991; Proc. Radiation Research: A Twentieth-Century Perspective, Vol. II: Congress Proceedings, Academic Press (1992)

LITTLEFIELD, L. G., ${ }^{*}$ A. M. SAYER, ${ }^{*}$ F. SALLAM, ${ }^{*}$ E. L. FROME, AND R. A. KLEINERMAN, * "Experience with the 'Cytochalasin-B Method' for Quantifying Radiation-Induced Micronuclei in Human Lymphocytes," pp. 183-188. (1.103)

Oral Presentation, 2nd International Conference on Indust and Applied Math, Washington, DC, July 8-12, 1991

WORLEY, P. H., "Modeling Histogram Data with Piecewise Polynomials." 
Fifth Annual Workshop on Space Operations, Applications, and Research (SOAR '91), NASA/Johnson Space Center, Houston, TX, July 9-11, 1991; Proc. NASA CP.S127, Vol. I (1991)

MANN, R. C., K. FUJIMURA, AND M. A. UNSEREN, "Research and Development at ORNL/CESAR Towards Cooperating Robotic Systems for Hazardous Environments," pp. 402-406. (3.4)

Fourth International Conference on Genetic Algorithm, San Diego, CA, July 19-16, 1991; Proc. Belew and Booker, Eds., Morgan Kaufmann Publishers (1991)

LIEPINS, G. E., AND L. A. WANG," "Classifier System Learning of Boolean Concepts." (3.38)

VOSE, M. D., ${ }^{*}$ AND G. E. LIEPINS, "Schemata Disruption." (3.41)

Conference on Numerical Methods in Engineering Problems, Caracas, Venezuela, July 15-17, 1991

NG, E., AND B. W. PEYTON, "Parallel Solution of Sparse Symmetric Positive Definite Linear Systems."

Oral Presentation, Universidad Central de Oriente, Oriente, Venezuela, July 18-19, 1991

NG, E., AND B. W. PEYTON, "Parallel Solution of Sparse Symmetric Positive Definite Linear Systems."

Oral Presentation, Universidad Central de Venezuela, Caracas, Venezuela, July 1826, 1991

NG, E., "Direct Solution of Sparse Linear Systems."

19th IMACS World Congress on Computation and Applied Mathematics, Dublin, Ireland, July 21-26, 1991; Proc. Vol. 1 (1991)

GRAY, L. J., AND C. S. SOUCIE,* "Hermite Interpolation Algorithm for Evaluating Hypersingular Boundary Integrals," pp. 240-241. (1.53)

JESSUP, E. R., "A Divide and Conquer Approach to the Nonsymmetric Eigenvalue Problem." (1.33)

Oral Presentation, National Research Council's Subcommittee on A-Bomb Dosimetry, Washington, DC, July 25, 1991

LARSON, D. C., "Nitrogen Cross-Section Measurements at ORNL."

IEEE International Conference on Systems Engineering, Dayton, OH, August 1-9, 1991; Proc. (1991)

UNSEREN, M. A., "Dynamic Coupling Effects in Modeling and Control of Hard Contact Motion of a Manipulator," pp. 100-104. (3.10) 
Oral Presentation, Solenoidal Detector Collaboration Meeting, Lawrence Berkeley Laboratory, Berkeley, CA, August 5, 1991

GABRIEL, T. A., "Compensation and Energy Resolution."

1991 Joint Statistical Meetings, Atlanta, GA, August 19-22, 1991

MORRIS, M. D., T. J. MITCHELL, AND D. YLVISAKER,* "Bayesian Design and Analysis of Computer Experiments: Use of Derivatives in Surface Prediction." (1.84)

Boundary Element Methods-19 Conference, Tulsa, OK, August 21-29, 1991

LUTZ, E., * L. J. GRAY, A. R. INGRAFFEA, "An Overview of Integration Methods for Hypersingular Bound.ary Integ-als." (1.56)

Human Factors Society Annual Meeting, San Francisco, CA, September 2-6, 1991; Proc. Vol. 2 (1991)

KNEE, H. E., AND R. J. CARTER, "A Research Profile of the Cognitive Systems and Human Factors Group at the Oak Ridge National Laboratory," pp. 1175-1179. (3.42)

Oral Presentation, Fourth SIAM Conference on Applied Linear Algebra, Minneapolis, MN, September 11-14, 1991

DONGARRA, J. J., G. A. GEIST, AND C. H. ROMINE, "Calculating the Eigenvalues and Eigenvectors for a General Matrix Reduced to Tridiagonal Form."

NG, E., AND B. W. PEYTON, "A Supernodal Cholesky Factorization Algorithm for Shared-Memory Multiprocessors."

NG, E., AND B. W. PEYTON, "Block Sparse Cholesky Algorithms on Advanced Uniprocessor Computers."

95th Annual Human Factors Society Meeting, San Francisco, CA, September 19-16, 1991

TERRANOVA, M., D. HARTLEY, ${ }^{*}$ B. G. COURY, ${ }^{*}$ AND K. N. HOOPER,* "Individual and Team Communication in a Dynamic Task." (3.43)

American Nuclear Society Meeting on Frontiers in Innovative Computing for the Nuclear Industry, Jackson, WY, September 15-18, 1991; Trans. Am. Nucl. Soc. 11 (1991)

SWEENEY, F. J., M. BECKERMAN, P. L. BUTLER,* J. P. JONES, AND D. B. REISTER, "Application of Autonomous Robotics to Surveillance of Radioactive Waste Storage Containers for Surface Contamination," p. 724.

Oral Presentation, Minisymposium on Linear Algebra, University of Tennessee, Knoxville, TN, September 27, 1991

D'AZEVEDO, E. F., W.-P. TANG, * AND P. A. FORSYTF,, "Recent Results on Ordering for PCG Methods." 
DONGARRA, J. J., G. A. GEIST, AND C. H. ROMINE, "Calculating the Eigenvalues and Eigenvectors for a General Matrix Reduced to Tridiagonal Form."

Oral Presentation, Second Workshop on Numerical Solutions of Fluid Flow in Spherical Geometry, Boulder, CO, October 9-11, 1991

DRAKE, J. B., D. W. WALKER, AND R. E. FLANERY, "Parallel SemiLagrangian Transport for MIMD Architectures."

Institute for Mathematics and Its Applications Workshop on Sparse Matrix Computations: Graph Theory, Issues and Algorithms, Minneapolis, MN, October $14-18,1991$

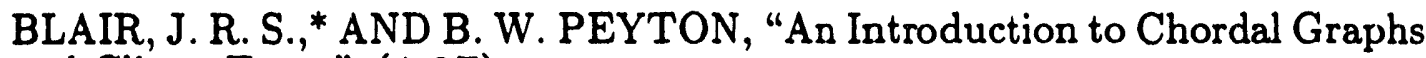
and Clique Trees." (1.37)

BLAIR, J. R. S., ${ }^{*}$ AND B. W. PEYTON, "On Finding Minimum-Diameter Clique Trees," (oral presentation only).

GILBERT, J. R.,* AND E. G. NG, "Predicting Structure in Nonsymmetric Sparse Matrix Factorizations." (1.39)

GILBERT, J. R., ${ }^{*}$ E. G. NG, AND B. W. PEYTON, "An Efficient Algorithm to Compute Row and Column Counts for Sparse Cholesky Factorization," (oral presentation only).

NG, E., "On the Structure of Sparse Gaussian Elimination with Partial Pivoting," (oral presentation only).

Oral Presentation, Heterogeneous Network-Based Concurrent Computing Workshop, Tallahassee, FL, October 16-18, 1991

GEIST, G. A., "PVM and HeNCE: A Software Environment for Programming Distributed Heterogeneous Computers."

Oral Presentation, KAPL/BETTIS/ORNL Discrete Ordinates Development Coordination Meeting, Knolls Atomic Power Laboratory, Schenectady, NY, October 17, 1991

RHOADES, W. A., "TORT Improvement Activities - FY 91."

Topical Conference on Nuclear Data for Fusion Reactor Technology, Karlsruhe, Germany, October 23, 1991; Proc. (1992)

LARSON, D. C., and C. Y. FU, "Potential Improvements to ENDF/B-VI for Fusion Data," pp. 113-118. (2.33)

Oral Presentation, Biostatistics Department, University of Arizona, Tucson, AZ, October 24, 1991

BOWMAN, K. O., L. R. SHENTON,* AND M. A. KASTENBAUM,*

"Discrete Pearson Distributions." 
Oral Presentation, Parallel Circus Workshop, Oak Ridge, TN, October 25, 1991 WORLEY, P. H., "Parallelizing Across Time When Solving Time-Dependent PDEs."

International Conference on Fast Reactors and Related Fuel Cycles, Kyoto, Japan, October 28-November 1, 1991; Proc. Vol. 1 (1991)

KNEE, H. E., AND J. C. SCHRYVER, "Models of Human Operators: Their Need and Usefulness for Improvement of Advanced Control Systems and Control Rooms," pp. 4.5-1 - 4.5-10. (3.48)

Oral Presentation, 99rd Annual Meeting of the American Physical Society, Division of Plasma Physics, Tampa, FL, November 4-8, 1991

LYNCH, V. E., ${ }^{*}$ B. A. CARRERAS, ${ }^{*}$ J. B. DRAKE, J. N. LEBOEUF, ${ }^{*}$ AND D. W. WALKER, "The Performance of a Fluid Code on a Hypercube and Connection Machine."

1991 Winter Meeting of the American Nuclear Society, San Francisco, CA, November 10-15, 1991; Trans. Am. Nucl. Soc. 64 (1991)

AZMY, Y. Y., "Optimal Diffusion Synthetic Acceleration of Discrete Ordinates Transport Calculations," p. 284.

DE SAUSSURE, G., AND N. M. LARSON,* "Analysis of ${ }^{249} \mathrm{Cf}$ Neutron Cross Sections in the Resolved Resonance Region," p. 540.

DIFILIPPO, F. C., "Influence of the Angular Correlation of Fission Neutrons on Noise Signatures," p. 299.

INGERSOLL, D. T., "Initial Phase II Testing of ENDF/B-VI Shielding Data," p. 564.

MAERKER, R. E., AND M. L. WILLIAMS,* "Improvements in Calculational Technique in Pressure Vessel Dosimetry," p. 489.

SWEENEY, F. J., "Random and Systematic Errors in Laser Range Camera Data," p. 621.

WRIGHT, R. Q.,* AND J. E. WHITE, "Fast Reactor Data Testing of ENDF/B-VI at ORNL," p. 566.

Oral Presentation, Southeast Section American Physical Society (SESAP) Meeting, Duke University, Durham, NC, November 11-19, 1991

GABRIEL, T. A., "Overview of the Superconducting Super Collider Project."

JONES, D. W., ${ }^{*}$ D. R. FRANCESCHETTI,* B. W. CAMPBELL,* J. D. TURNER,* AND T. A. GABRIEL, "Energy Deposition by Electrons in Particle Detectors."

MOORE, B.," AND T. A. GABRIEL, "Calorimetry Simulation Using CALOR89 on UNIX Computers." 
Oral Presentation, Distributed Computing Workshop, University of Utah, Salt Lake City, UT, November 14-15, 1991

GEIST, G. A., "Using the PVM System: A Case Study."

GEIST, G. A., "The PVM System for Distributed Heterogeneous Corcputing."

Supercomputing '91 Conference, Albuquerque, NM, November 18-22, 1991; Proc. (1991)

BEGUELIN, A., J. J. DONGARRA, G. A. GEIST, R. MANCHEK, AND V. S. SUNDERAM,* "Graphical Development Tools for Network-Based Concurrent Supercomputing," pp. 435-445. (1.2)

GEIST, G. A., "Parallel Superconductor Code Exceeds 2.5 Gflops on iPSC/860." (1.10)

GEIST, G. A., "PVM and HeNCE: Software for Distributed Computing," (oral presentation only).

Oral Presentation, IAEA Advisory Group Meeting on FENDL-2 and Associated Benchmark Calculations, Vienna, Austria, November 18-22, 1991

LARSON, D. C., AND C. Y. FU, "Potential Improvenents to ENDF/B-VI for Structural Materials."

Minisymposium on Computing Climate Change: Can We Beat Natvre? Supercomputing '91, Albuqierque, NM, November 21, 1991

DRAKE, J. B., D. W. WALKER, AND P. H. WORLEY, "Atmospheric Spectral Models on MIMD and SIMD Architectures." (1.74)

Oral Presentation, International Workshop on Industrial Applications of Fuzzy Control and Intelligent Systems, College Station, TX, November 21-22, 1991

PIN, F. G., AND H. WATANABE,* "Application of Custom-Designed VLSI Fuzzy Inferencing Hardware to the Sensor-Based Navigation of an Autonomous Mobile Robot."

Oral Presentation, Math Department, University of Tennessee, Knoxville, TN, November 22, 1991

BOWMAN, K. O., L. R. SHENTON,* AND M. A. KASTENBAUM,* "Discrete Pearson Distributions."

Materials Research Society 1991 Fall Meeting, Boston, MA, December 2-6, 1991; Proc. Mat. Res. Soc. Symp. Vol. 239 (1992)

PAWEL, J. E., ${ }^{*}$ W. E. LEVER, D. J. DOWNING, C. J. MCHARGUE, ${ }^{*}$ L. J. ROMANA,* AND J. J. WERT,* "Using Weibull Statistics to Analyze Ion Beam Enhanced Adhesion as Measured by the Pull Test," p. 541. (1.110) 
Oral Presentation, Executive Council, Oak Ridge, TN, December 9, 1991 GEIS' ${ }^{\prime}$ ', G. A., "Solving Computational Grand Challenges Using PVM and a Network of Computers."

Handouts, Defense Nuclear Agency 2nd Annual Human Response Program (BRP) Technology Transfer Meeting, Arlington, VA, December S-5, 1991

SANTORO, R. T., "Radiation Environments Program."

90th IEEE Conference on Decision and Control, Brighton, England, December 1119, 1991; Proc. Vol. II (1991)

LENHART, S. M., V. PROTOPOPESCU, AND S. STOJANOVIC,* "A TwoSided Game for Non-Local Competitive Systems with Control on Source Terms," pp. 1838-1839. (1.55)

Oral Presentation, 2nd International Workshop on Software Engineering, AI, and Neural Nets for High Energy and Nuclear Physics, L'Agelonde, France, January $13-18,1992$

GLOVER, C. W., C. Y. FU, AND T. A. GABRIEL, "ORNL Neural Net Research for Particle Tracking in Detector Systems."

Oral Presentation, The Workshop on Parallel Computing for $3 D$ Plasma Simulation, Phillips Laboratory, Albuquerque, NM, January 15, 1992

WALKER, D. W., "Problems with Parallel PIC and Related Matters."

Oral Presentation, Geophysical Fluid Dynamics Laboratory, Princeton, NJ, January 16, 1992

DRAKE, J. B., "PCCM: A Distributed-Memory Parallel Implementation of the Community Climate Model."

Oral Presentation, Ninth Meeting of the DOE Performance Assessment Task Team, Augusta, GA, January 21, 1992

WORLEY, B. A., "Sensitivity and Uncertainty Analysis Methods Used in Performance Assessment of Waste Storage and Isolation Systems."

Oral Presentation, Alabama Supercomputer Center Seminar Series, Huntsville, $A L$, January 30, 1992

GEIST, G. A., "Getting High Performance Through Parallel Programming."

Oral Presentation, Human Factors Experimental Research Facility, Washington, DC, January 30, 1992

KNEE, H. E., "Human Factors Research at Oak Ridge National Laboratory and Ideas for a Human Performance Research Program." 
Lecture, University of Tennessee, Computer Science Department, Knoxville, TN, January 30, 1992

WALKER, D. W., "Techniques for Dynamically Load Balancing Parallel Particle-In-Cell Simulations."

Oral Presentation, TORT Three-Dimensional Discrete Ordinates Neutron/Photon Transport Code Workshop, February 9-6, 1992

BURNS, T. J., "Graphical Tools for Modeling, Debugging, and Display."

ENGLE, W. W., "Discrete Ordinates - Background."

KIRK, B. L., "Sample Problems."

LILLIE, R. A., "Source Input."

LILLIE, R. A., "Procedures for Problem Solution Activity."

MANNESCHMIDT, J., "System Implementation."

PACE, J. V., "Description of Problem(s) to be Solved."

RHOADES, W. A., "TORT - Background, Description, and Applications."

RHOADES, W. A., "Control Arrays."

RHOADES, W. A., "Source Iteration Strategies."

SLATER, C. O., "Space Mesh, Geometry, Quadrature Sets, and Capability."

SLATER, C. O., "Graphics Display of Flux/Response Data."

WHITE, J. E., "Cross-Section Data."

WILLIAMS, L. R., "Key Flux and Response Output - Cross-Section Requirements/Preparation."

Oral Presentation, Clarkson University, Potsdam, NY, February 10, 1992

GRAY, L. J., "Hypersingular Boundary Integral Equations."

Oral Presentation, Cornell University, Ithaca, NY, February 11, 1992

GRAY, L. J., "Hermite Interpolation for Hypersingular Boundary Integrals."

Oral Presentation, WATTec Conference, Knoxville, February 19, 1992

MANN, R. C., "Robotics and Related Intelligent Systems for the Space Exploration Initiative." 
First Annual Conference on Evolutionary Programming, La Jolla, CA, February 21-22, 1992; Proc. (1992)

MCDONNELL, J. R., ${ }^{*}$ B. L. ANDERSEN,* W. C. PAGE,* AND F. G. PIN, "Mobile Manipulator Configuration Optimization Using Evolutionary Programming," pp. 52-62. (3.16)

Spring Meeting of Nuclear Physics Sections, University of Salzburg, Austria, February 24-28, 1992

SCHMIEDMAYER, J., ${ }^{*}$ P. RIEHS, ${ }^{*}$ J. A. HARVEY, AND N. W. HILL,* "Electric Properties of the Neutron from Precision Cross Section Measurements." (2.12)

Eighth IEEE Conference on Artificial Intelligence for Applications, Monterey, CA, March 2-6, 1992; Proc. (1992)

GUAN, X., R. J. MURAL, ${ }^{*}$ J. R. EINSTEIN, R. C. MANN, AND E. C. UBERBACHER, "GRAIL: An Integrated Artificial Intelligence System for Gene Recognition and Interpretation," pp. 9-13. (3.51)

American Nuclear Society Topical Meeting on Advances in Reactor Physics, Charleston, SC, March 8-11, 1992; Proc. (1992)

AZMX, Y. Y., "A Nodal Integral Method for Neutron Diffusion In Hexagonal Geometry," Vol. 1, pp. 509-519. (4.59)

PRIMM, R. T., III, "Calculation of Critical Experiment Parameters for the High Flux Isotope Reactor," Vol. 2, pp. 203-218. (4.20)

WARD, R. C., "A DOE Partnership for Grand Challenge Applications," (oral presentation only).

Computer Hardware Advanced Mathematics and Model Physics Science Team Meeting, Las Vegas, NV, March 17, 1992

DRAKE, J. B., D. L. WILLIAMSON,* AND I. FOSTER,* "Parallel Algorithms for the Spectral Transform and Semi-Lagrangian Transport Methods." (1.75)

DOE/ORAU Speaker's Bureau at the University of New Orleans, New Orleans, LA, March 19, 1992

SPELT, P. F., "Autonomous Mobile Robots to Cope with Complex Hostile Environments."

Oral Presentation, Sixth International Parallel Processing Symposium, Beverly Hills, CA, March 29-26, 1992

DONGARRA, J. J., "A Look at the Evolution of Software for Numerical Linear Algebra." 
Oral Presentation, The Systems Research Center, University of Maryland, College Park, MD, March 27, 1992

MANN, R. C., "Robotics and Intelligent Systems Research at ORNL/CESAR."

Third Annual Combat Vehicle Survivability Symposium, Gaithersburg, MD, March 31-April 2, 1992

HAALAND, C. M., J. O. JOHNSON, R. T. SANTORO, AND K. G. KERRIS,* "Calculation of Protection Factors of the M1A1 Tank Against Initial Nuclear Radiation." (4.46)

KEHLET, R. A., ${ }^{*}$ R. W. YOUNG, ${ }^{*}$ C. M. WARD, ${ }^{*}$ H. G. SNYDER, ${ }^{*}$ C. N. DAVIDSON, * R. T. SANTORO, J. O. JOHNSON, AND T. J. BURNS, "Combined Conventional and Radiation Protection." ( )

Oral Presentation, 1992 IMSL User Groups for Europe, Munich, Germany, April $1-3,1992$

DONGARRA, J. J., "A Look at the Evolution of Software for Numerical Linear Algebra."

Oral Presentation, CHAMMP (Computer Hardware Advanced Mathematics and Model Physics) Directed Team Meeting, Santa Fe, NM, April 3, 1992

DRAKE, J. B., R. E. FLANERY, I. FOSTER,* AND J. MICHALAKES,* "Progress on PCCM2: Implementation and Performances Issues."

Oral Presentation, IBM-SUPER Conference, Penn State University, University Park, PA, April 5-8, 1992

DONGARRA, J. J., "Heterogeneous Network Computing Environments: Trends and Issues."

Oral Presentation, American Nuclear Society Executive Conference on Remote Operations and Robotics in Nuclear Industry-II, Pine Mountain, GA, April 5-8, 1992

MANN, R. C., "DOE Basic Research in Intelligent Machines."

SWEENEY, F. J., "Robotics for Future Nuclear Reactor Operations."

International Sherwood Fusion Theory Conference, Santa Fe, NM, April 6-8, 1992 LYNCH, V. E., ${ }^{*}$ B. A. CARRERAS,* J. B. DRAKE, J. N. LEBOEUF,* P. LIEWER, * AND D. W. WALKER, "The Performance of a Fluid Code on Massively Parallel Machines," (1.58)

Oral Presentation, Computer Hardware Advanced Mathematics and Model Physics Development Meeting, Santa Fe, NM, April 6-8, 1992

WORLEY, P. H., "Parallel Spectral Transform Methods: Options and New Directions." 
Copper Mountain Conference on Iterative Methods, Copper Mountain, CO, April 9-14, 1992

DONATO, J. M., "Iterative Methods for Nonsymmetric Coupled Systems." (1.66)

DONATO, J. M., "The Fourier Analysis Technique and $\epsilon$-PseudoEigenvalues." (1.67)

Lecture, Oregon Graduate Institute, Portland, OR, April 1C, 1992

WALKER, D. W., "ScaLAPACK: A Scalable Linear Algebra Library for Distributed Memory Concurrent Computers."

Oral Presentation, Material Modeling Conference, Morganton, WV, April 12-14, 1992

GEIST, G. A., "Distributed Computing of the Electronic Structure of Disordered Alloys."

Oral Presentation, Computer Science Department, Radford University, Radford, VA, April 20, 1992

NG, E., "Solving Linear Systems on Multiprocessor Systems."

1992 Joint April Meeting of the American Physical Society and the American Association of Physics Teachers, Washington, DC, April 20-24, 1992; Proc. Vol. 97(2) (1992)

CARLTON, R. F., ${ }^{*}$ C. H. JOHNSON, ${ }^{*}$ AND J. A. HARVEY, "R-Matrix Analysis of an ORELA Measurement of the $n+{ }^{48} \mathrm{Ca}$ Total Cross Section from $100 \mathrm{keV}$ to $3.9 \mathrm{MeV}$," p. 902.

Oral Presentation, Department of Physics, Lowell University, Lowell, MA, April 22, 1992

DICKENS, J. K., "Research with Neutrons at the Oak Ridge Electron Linear Accelerator (ORELA) Facility."

Scalable High Performance Computing Conference, Williamsburg, VA, April 26-29, 1992; Proc. J. Saltz and R. Voigt, Eds., IEEE Computer Society Press (1992)

BEGUELIN, A., J. J. DONGARRA, G. A. GEIST, R. MANCHEK,* K. MOORE, ${ }^{*}$. WADE,* AND V. SUNDERAM,* "HeNCE: Graphical Development Tools for Network-Based Concurrent Computing." (1.3)

DONGARRA, J., R. VAN DE GEIJN,* AND D. W. WALKER, "A Look at Scalable Dense Linear Algebra Libraries." (1.32)

WORLEY, P. H., "Phase Modeling of a Parallel Scientific Code," pp. 322327. (1.21) 
American Nuclear Society Topical Meeting on New Horizons in Radiation Protection and Shielding, Pasco, WA, April 26-90, 1992; Proc. (1992)

ALSMILLER, F. S., R. G. ALSMILLER, JR., AND O. W. HERMANN,* "The Use of Delay Times in the Intranuclear Cascade Model of High Energy $(\sim 5 \mathrm{GeV})$ Hadron-Nucleus Collisions," pp. 505-508. (4.27)

ALSMILLER, JR., R. G., AND F. S. ALSMILLER, "Particle Production Models in HETC88 in the Energy Range 3 to $30 \mathrm{GeV}$," pp. 499-504. (4.28)

BURNS, T. J., AND M. S. SMITH, "Graphical Debugging of Combinatorial Geometry," pp. 48-55. (4.66)

INGERSOLL, D. T., "The Horizon for Advanced Reactor Shielding Technology: Sunrise or Sunset?" (oral presentation only).

INGERSOLL, D. T., R. Q. WRIGHT, * AND C. O. SLATER, "Phase II Testing of ENDF/B-VI Shielding Data," pp. 385-391. (4.17)

JOHNSON, J. O., T. J. BURNS, J. D. DRISCHLER, AND J. M. BARNES,* "Nuclear Vulnerability of the U.S. M60A1 Tank in an Initial Radiation Environment: MASH Code System Analysis," pp. 267-274. (4.47)

JOHNSON, J. O., J. D. DRISCHLER, AND J. M. BARNES,* "Analysis of the Fall-1989 Two-Meter Box Test Bed Experiments Performed at the Army Pulse Radiation Facility (APRF)," pp. 251-258. (4.48)

KIRK, B. L., Y. Y. AZMY, T. A. GABRIEL, AND C. Y. FU, "Implementation of the Monte Carlo Charged Particle Transport Code EGS4 on the Hypercube," pp. 34-37. (5.1)

LARSON, D. C., C. L. DUNFORD,* AND C. NORDBORG,* "NEACRP/ NEANDC Working Group on International Evaluation Cooperation," pp. 392-399. (2.32)

PACE, J. V., III, D. T. INGERSOLL, F. J. MUCKENTHALER, R. R. SPENCER, H. T. HUNTER, AND A. SHONO, "Current Status of JASPER: Japanese-American Shielding Program of Experimental Research," (oral presentation only).

Seminar, College of Engineering, Iowa State University, Ames, IA, April 27, 1992 BOWMAN, K. O., "Discrete Pearson Distributions."

International Conference on Radiation-Tolerant, Florida State University, Tallahassee, FL, April 28-May 2, 1992

GABRIEL, T. A., T. HANDLER, * AND P. K. JOB,* "Comparison of Experimental and Calculational Energy Deposition Profiles in Calorimeters and Other Damage Related Data," (4.32) 
Oral Presentation, Rover/Mobility Systems Workshop, Johnson Space Center, Houston, TX, April 29-90, 1992

MANN, R. C., "Real-Time Qualitative Reasoning and Communication Technologies for Tele-Robotic Systems."

Oral Presentation, Workshop on Standards for Message Passing in a Distributed Memory Environment, Williamsburg, VA, April 29-90, 1992

WORLEY, P. H., "PICL: Description, Experiences, and Implications for Message-Passing, Interface Standards."

Oral Presentation, Statistics Program, Mathematical Sciences Research Institute, Berkeley, CA, May 9-16, 1992

MORRIS, M. D., AND A. D. SOLOMON, "Design and Analysis for an Inverse Problem of Advective - Dispersive Fluid Flow."

Oral Presentation, Real Application on Parallel Systems, Workshop on Parallel Programming Models and Interfaces, Bonn, Germany, May 4, 1992

WALKER, D. W., "Standards for Message Passing on Distributed Memory Computers: the US View."

1992 IEEE International Conference on Robotics and Automation, Nice, France, May 12-14, 1992; Proc. (1992)

BECKERMAN, M., "A Bayes-Maximum Entropy Method for Multi-Sensor Data Fusion," pp. 1668-1674. (3.37)

FUJIMURA, K.,* "On Motion Planning Amidst Transient Obstacles," pp. 1488-1493. (3.19)

JONES, J. P., A. L. BANGS, AND P. L. BUTLER,* "A System for Simulating Shared Memory in Heterogeneous Distributed-Memory Networks with Specialization for Robotics Applications." pp. 2738-2744. (3.7)

JONES, J. P., P. L. BUTLER, ${ }^{*}$ M. BECKERMAN, AND F. J. SWEENEY, "Autonomous Surveillance for Surface Radiation Contamination," p. 63. (3.13)

KILLOUGH, S. M., * AND F. G. PIN, "Design of an Omnidirectional and Holonomic Wheeled Platform Prototype," pp. 84-90. (3.15)

PIN, F. G., H. WATANABE,* J. SYMON,* AND R. S. PATTAY, "Autonomous Navigation of a Mobile Robot Using Custom-Designed Qualitative Reasoning VLSI Chips and Boards," pp. 123-128. (3.24)

Oral Presentation, Institute of Chemical Research, University of Kyoto, Kyoto, Japan, May 22, 1992

UBERBACHER, E. C., R. MURAL, ${ }^{*}$ J. R. EINSTEIN, X. GUAN, AND R. C. MANN, "High-Speed Pattern Recognition and Analysis of DNA Sequences." 
International Symposium on Numerical Transport Theory, Moscow, Russia, May 29-91, 1992; Proc. (1992)

AZMY, Y. Y., "General Order Characteristic Methods for Solving Neutron Transport Problems," p. 49. (4.63)

Specialist Meeting on Application of Artificial Intelligence and Robotics to Nuclear Plants (AIR '92), Ibaraki-ken, Japan, May 25-27, 1992; Proc. (1992)

MANN, R. C., "CESAR Robotics and Intelligent Systems Research for Nuclear Environments," pp. 110-119. (3.1)

The NEANSC Specialists' Meeting on Fission Product Nuclear Data, JAERI Tokai Research Establishment, Ibaraki-ken, Japan, May 28-29, 1992; Proc. (1992)

LARSON, D. C., AND R. L. MACKLIN, "Review of Fission Product Capture Measurements at the Oak Ridge Electron Linear Accelerator (ORELA)," pp. 127-138. (2.6)

Oral Presentation, Fourth Meeting of the NEANSC Working Group on International Evaluation Cooperation, Mito, Japan, May 28-29, 1992

FU, C. Y., "Status Report to the Working Group on International Evaluation Cooperation from Subgroup I: Comparison of ENDF/B-VI, JEF-2/EFF-2, and JENDL-3 for ${ }^{52} \mathrm{Cr},{ }^{56} \mathrm{Fe}$, and ${ }^{38} \mathrm{Ni}$."

LARSON, D. C., "Status Report to the NEANSC Working Group on International Evaluation Cooperation from Subgroup II: Comparison of the Resonance Regions for ${ }^{52} \mathrm{Cr},{ }^{56} \mathrm{Fe}$, and ${ }^{58} \mathrm{Ni}$."

Second International Conference on Bioinformatics, Supercomputing, and Complex Genome Analysis, St. Petersburg Beach, FL, June 4-7, 1992

UBERBACHER, E. C., J. R. EINSTEIN, X. GUAN, AND R. J. MURAL, "Gene Recognition and Assembly in the GRAIL System: Progress and Challenges." (3.54)

1992 Summer Meeting of the American Nuclear Society, Boston, MA, June 7-12, 1992; Trans. Am. Nucl. Soc. 65 (1992)

DIFILIPPO, F. C., B. A. WORLEY, AND T. WILLIAMS,* "Analysis of Kinetics Experiments in LEU-HTR Configurations of the PROTEUS Facility," pp. 457-459.

HAIRE, M. J., * B. E. LEWIS, * S. C. NELSON,* AND M. S. SMITH, "Dissolution System Reliability Data Base," pp. 379-380.

KALLFELZ, J. M., * J. E. HORWEDEL, * AND B. A. WORLEY, "Sensitivity Analysis of Reactor Safety Parameters with Automated Adjoint Function Generation," pp. 218-220.

PRIMM, R. T., III, "Prediction and Measurement of Fuel Depletion Due to Irradiation of HFIR Fuel Elements," pp. 446-448. 
Informal Seminar, Institute for Mathematics and Its Applications (IMA), Minneapolis, MN, June 9, 1992

DONATO, J. M., "Comparison of Iterative Methods for Nonsymmetric Coupled Elliptic Equations."

Oral Presentation, CERFACS (European Centre for Research and Advanced Training and Scientific Computation) Training Cycle, Toulouse, France, June 9, 1992

DONGARRA, J. J., "An Overview of High-Performance Computers."

9th International Conference on Computational Methods in Water Resources, Denver, CO, June 9-12, 1992; Proc. Vol. 1 (1992)

ELNAWAWY, O. A., * AND Y. Y. AZMY, "The Cell Analytical-Numerical (CAN) Method for the Solution of the Richards Equation," p. 203. (4.68)

7th International Association for Mathematics and Computer Simulation Conference on Computer Methods for Partial Differential Equations, New Brunswick, NY, June 21-24, 1992; Proc. (1992)

KAHANER, D. K., ${ }^{*}$ E. G. NG, W. E. SCHIESSER, ${ }^{*}$ AND S. THOMPSON, ${ }^{*}$ "Experiments with an Ordinary Differential Equation Solver in the Parallel Solution of Method of Lines Problems on a Shared Memory Parallel Computer, Part II," pp. 404-410. (1.35)

LAWKINS, W. F., AND J. S. PAYNE,* "An Implementation of a Parallel MOL Solver on the Intel Gamma Parallel Computer." (1.71)

ROTHBERG, E., ${ }^{*}$ A. GUPTA, ${ }^{*}$ E. NG, AND B. W. PEYTON, "Parallel Sparse Cholesky Factorization Algorithms for Shared-Memory Multiprocessor Systems," pp. 622-628. (1.46)

ASME Pressure Vessels and Piping 1992 Summer Conference, New Orleans, LA, June 21-25, 1992

POTYONDY, D. O., ${ }^{*}$ L. J. GRAY, AND A. INGRAFFEA,* "Simulation of 3D Non-Planar Fatigue Crack Growth in a Turbine Blade Root." (1.60)

Oral Presentation, Mechanical Engineering Lab, Tsukuba, Japan and Other Japanese Laboratories, June 21-August 90, 1992

BANGS, A. L., AND J. P. JONES, "Research at the University of Tennessee and Oak Ridge National Laboratory."

O-al Presentation, Los Alamos High Performance Computing Workshop, Albuquerque, NM, June 22, 1992

GEIST, G. A., "PVM Overview." 
Oral Presentation, NATO Advanced Research Workshop on Software for Parallel Computation, Centraro, Italy, June 22-26, 1992

DONGARRA, J. J., "Graphical Development Tools for Network-Based Concurrent Computing."

IEEE/IES Intelligent Vehicles '92, Detroit, MI, July 1-2, 1992

BANGS, A., F. G. PIN, AND S. M. KILLOUGH,* "An Implementation of Redundancy Resolution and Stability Monitoring for a Material Handling Vehicle." (3.12)

Oral Presentation, Conference on Supercomputing in Science and Industry, Monte Verita, Switzerland, July 2-5, 1992

DONGARRA, J. J., "LAPACK: A Package of Mathematical Software for High-Performance Computers."

1992 IEEE International Conference on Intelligent Robots and Systems, Raleigh, NC, July 7-10, 1992; Proc. (1992)

PIN, F. G., H. WATANABE, ${ }^{*}$ J. SYMON, ${ }^{*}$ AND R. S. PATTAY, "Using Custom-Designed VLSI Fuzzy Inferencing Chips for the Autonomous Navigation of a Mobile Robot," pp. 790-795. (3.22)

Oral Presentation, Center for European Nuclear Research, Geneva, Switzerland, July 9, 1992

DONGARRA, J. J., "Graphical Development Tools for Network-Based Concurrent Computing."

Oral Presentation, IBM Europe Institute Workshop on Highly Parallel Computers, Oberlech, Austria, July 19-1\%, 1992

BEGUELIN, A., J. J. DONGARRA, G. A. GEIST, R. MANCHEK,* K. MOORE,* R. WADE,* AND V. S. SUNDERAM,* "PVM, HeNCE, and XAB: Graphical Development Tools for Network-Based Concurrent Computing."

1992 IEEE Annual Conference on Nuclear and Space Radiation Effects, New Orleans, LA, July 19-17, 1992

TSVEYBAK, I., * W. BUGG, ${ }^{*}$ J. A. HARVEY, AND J. WALTER, * "Fast Neutron-Induced Changes in Net Impurity Concentration of High-Resistivity Silicon." (2.15)

Test Technology Symposium V, Johns Hopkins University, Applied Physics Laboratory, Laurel, MD, July 14-16, 1992

JOHNSON, J. O., R. T. SANTORO, T. J. BURNS, AND R. A. KEHLET,* "Analytic Methods for Radiation Environment Simulation and Vulnerability Assessment." (4.51) 
Oral Presentation, Environmental Working Group Uncertainties Panel (Radiation Subpanel), Nichols Research Center, Huntsville, AL, July 16, 1992

SANTORO, R. T., "Radiation Environments."

1992 SPIE Conference on Neural and Stochastic Methods in Image and Signal Processing, San Diego, CA, July 20-29, 1992; Proc. Vol. 1766 (1992)

BECKERMAN, M., AND F. J. SWEENEY, "Restoration and Fusion of Laser Range Camera Images," pp. 454-465. (3.35)

SIAM 40th Anniversary Meeting, Los Angeles, CA, July 20-24, 1992

DONATO, J. M., "The Relationship Between $\epsilon$-Pseudo-Eigenvalues and the Fourier Analysis Techniques." (1.68)

DONATO, J. M., "Comparison of Iterative Methods for Nonsymmetric Coupled Elliptic Equations." (1.65)

GEIST, G. A., "Solving Material Science Problems at ORNL's Center for Computational Studies." (1.11)

GRAY, L. J., AND L. L. Manne,* "Hypersingular Integral Equations at a Corner." (1.52)

LENHART, S. M., "Environmental Modeling at Oak Ridge National Laboratory," (oral presentation only).

Oral Presentation, Joint DOE/JAERI MHTGR Program Meeting, Washington, DC, July 21-22, 1992

WORLEY, B. A., "Role of VHTRC Data in Validation of MHTGR Reactor Physics Codes."

Oral Presentation, 1992 International Nuclear Physics Conference, Wiesbaden, Germany, July 26-August 1, 1992

KOPECKY, S., ${ }^{*}$ J. A. HARVEY, N. W. HILL, ${ }^{*}$ AND P. RIEHS, "Investigations on the Neutron Charge Radius."

Oral Presentation, Gordon Conference, Plymouth, NM, July 27-91, 1992 GEIST, G. A., "Creation of a Nation-Wide Distributed Computer."

Oral Presentation, Radiation Environments Program Review, Munster, Germany, July 28-29, 1992

BURNS, T. J., "Graphical Tools for Geometry Modeling, Debugging, and Display."

JOHNSON, J. O., "MASH 1.0 A Monte Carlo Adjoint Shielding Code System." 
JOHNSON, J. O., J. M. BARNES,* J. D. DRISCHLER, R. T. SANTORO, AND T. J. BURNS, "Application of the MASH 1.0 Code System to Armored Vehicle Nuclear Vulnerability Analysis."

SANTORO, R. T., J. D. DRISCHLER, AND J. O. JOHNSON, "Resolution of Dose and Reduction Factor Questions in APRF Experiments: Renormalization of Calculated and Measured Data."

SANTORO, R. T., AND S. Y. WHITAKER,* "Review of Experiments for Benchmarking MASH - Version 1.0 Discussion of Measurements and Analyses."

IABEM-2 (International Association for Boundary Elements Meeting), Boulder, CO, A ugust 2-5, 1992

FLANERY, R. E., J. B. DRAKE, AND L. J. GRAY, "Boundary Elements on Distributed Memory Architectures." (1.69)

POTYONDY, D. O.,* L. J. GRAY, AND A. R. INGRAFFEA,* "Hermite Interpolation Algorithm for 2D Fracture Mechanics," (oral presentation only).

Oral Presentation, SOAR '92, Space Operations, Applications, and Research Symposium, Houston, TX, August 4-6, 1992

PIN, F. G., "Real Time Qualitative Reasoning for Telerobotic Systems."

99th International Field Emission Symposium, Halifax, Canada, August 10-14, 1992

BOWMAN, K. O., L. R. SHENTON,* AND M. K. MILLER,* "The Use of Moment Estimators to Determine the Parameters of Concentration Fluctuations in Random Area Atom Probe Analyses." (1.86)

MILLER, M. K., ${ }^{*}$ K. O. BOWMAN, A. CEREZO,* AND J. M. HYDE,* "Comparison of Models for Deconvoluting the Compositions of Coexisting Phases." (1.94)

American Nuclear Society Topical Meeting on Nuclear Technologies for Space Exploration, Jackson Hole, WY, August 16-19, 1992; Proc. Vol. III (1992)

BURNS, T. J., "Graphical-Based Construction of Combinatorial Geometries for Radiation Transport and Shielding Applications," pp. 717-727. (4.64)

CRAMER, S. N., "Analytic Estimation of Radiation Effects in Space Using Solid Angles," pp. 654-663. (5.2)

JOHNSON, J. O., R. T. SANTORO, J. D. DRISCHLER, AND J. M. BARNES,* "Radiation Protection for Human Exploration of the Moon and Mars: Application of the MASH Code System," pp. 697-706. (4.52) 
1992 ANS/ASME Nuclear Energy Conference, San Diego, CA, August 23-26, 1992; Proc. (1992)

HAIRE, M. J.,* M. S. SMITH, AND D. R. MOSER,* "Framework for Monitoring Reliability," pp. 179-182. (4.14)

WOOD, D. H., M. S. SMITH, AND J. D. DRISCHLER, "Motor-Operated Globe Valve Performance in a Liquid Sodium Environment," pp. 341-346. (4.24)

10th Symposium on Computational Statistics, Neuchatel, Switzerland, A ugust 2928, 1992; Proc. Computational Statistics, Vol. 1, Y. Dodge and J. Whittaker, Eds., Physics Verlag (1992)

OSTROUCHOV, G., "HModel: An X Tool for Global Model Search," pp. 269-274. (1.95)

Oral Presentation, Risk Methods Day sponsored by East Tennessee Society for Risk Analysis, SAIC, Oak Ridge, TN, August 25, 1992

FLANAGAN, D. M., "The Statistics Partner in Waste Management: Data Quality Objectives and Statistical Sampling Plans."

Oral Presentation, Nuclear Criticality Safety Workshop, Breckenridge, CO, August 27-28, 1992

PRIMM, III, R. T., "Validation Studies Related to the Storage of Waste from Nuclear Fuel Services in Building 3019 at Oak Ridge National Laboratory."

49rd Congress of the International Astronautical Federation, Washington, DC, A ugust 28-September 5, 1992

WALTON, J. T., ${ }^{*}$ N. A. HANNAN, ${ }^{*}$ K. R. PERKINS, ${ }^{*}$ J. J. BUKSA, ${ }^{*}$ B. A. WORLEY, AND D. DOBRANICH,* "System Model Development for Nuclear Thermal Propulsion." (4.23)

CNRS-NSF Collaborative Workshop on Environments and Tools for Parallel Scientific Computing, St. Hilaire du Touvet, France, September 7-8, 1992

CHOI, J., J. J. DONGARRA, AND D. W. WALKER, "Level 3 BLAS for Distributed Memory Concurrent Computers." (1.25)

CHOI, J., J. J. DONGARRA, AND D. W. WALKER, "The Design of Scalable Software Libraries for Distributed Memory Concurrent Computers." (1.24)

Oral Presentation, IBM Super Meeting, Umea, Sweden, September 14-16, 1992 DONGARRA, J. J., "Scalable High-Performance Libraries in Linear Algebra." DONGARRA, J. J., "PVM and HeNCE: Tools for Network-Based Concurrent Computing." 
Oral Presentation, C8T Seminar Series, K-25 Plant, Building 1007, Room 1070, Oak Ridge, TN, September 16, 1992

GEIST, G. A., "PVM Overview and Tutorial."

6th International Conference on Modeling Techniques and Tools for Computer Performance Evaluation, Edinbuigh, Scotland, September 16-18, 1992; Proc. (1992)

CARLSON, B. M., ${ }^{*}$ T. D. WAGNER, ${ }^{*}$ L. W. DOWDY, , AND P. H. WORLEY, "Speedup Properties of Phases in the Execution Profile of Distributed Parallel Programs," pp. 83-95. (1.5)

Conference on Computing in High-Energy Physics, Annecy, France, September 2125, 1992

GLOVER, C. W., C. Y. FU, T. A. GABRIEL, AND T. HANDLER,* "Charged Particle Track Reconstruction Using Artificial Neural Networks." (4.33)

HANDLER, T., ${ }^{*}$ E. NEIS, ${ }^{*}$ C. W. GLOVER, T. A. GABRIEL, AND S. SAINI,* "Feasibility of Using Neusal Networks as a Level 2 Calorimeter Trigger for Jet Tagging." (4.35)

Oral Presentation, Computer Science Department, University of Tennessee, Knoxville, TN, September 28, 1992

DONATO, J., "Comparison of Iterative Methods for Nonsymmetric Coupled Elliptic Equations."

1992 Annual Intel Supercomputer Users' Group Conference, Dallas, TX, October 4-7, 1992

SHELTON, W. A., AND G. A. CEIST, "Developing Large Scale Applications by Integrating PVM and the Intel iPSC/860." (1.16)

SINCOVEC, R. F., "Oak Ridge National Laboratory's Center for Computational Sciences," (oral presentation only).

WORLEY, P. H., "PICL: Past, Present, and Future." (1.22)

Computer Science Colloquim, Clemson University, Clemson, SC, October 7, 1992 BLAIR, J. R. S.* AND B. W. PEYTON, "On Finding Minimum-Diameter Cli . .e Trees." (1.38)

Nuclear Energy Agency Nuclear Science Committee Specialists Meeting on Evaluation and Processing of Covariance Data, Oak Ridge National Laboratory, Oak Ridge, TN, October 7-9, 1992; Proc. (1993)

HETRICK, D. M., * D. C. LARSON, AND C. Y. FU, "Approximate Methods for Generatior of Covariance Data for the Structural Materials of ENDF/BVI," pp. 173-182. (2.30) 
LARSON, N. M.,* "Covariances as Input to and Output from Resonance Analyses," pp. 221-236. (2.34)

MAERKER, R. E., B. L. BROADHEAD,* AND J. J. WAGSCHAL," "Needs for Evaluated Covariance Data for Reactor Pressure Vessel Dosimetry," pp. 13-32. (4.18)

SARTORI, E.,* AND R. W. ROUSSIN, "Computer Codes for Checking, Plotting and Processing of Neutron Cross-Section Covariance Data and Their Application," pp. 253-264. (5.12)

ZHAO, Z., ${ }^{*}$ C. Y. FU, AND D. C. LARSON, "Covariance Matrix of Model Parameters and Uncertainties of Calculated Neutron Cross Sections on ${ }^{19} \mathrm{~F}$," pp. 71-75. (2.38)

Symposium on Nuclear Data Evaluation Methodology, Brookhaven National Laboratory, Upton, NY, October 12-16, 1992

DIFILIPPO, F. C., J. P. RENIER,* AND B. A. WORLEY, "Status and Covariance Files of the Scattering Matrix of Graphite." (4.13)

FU, C. Y., "Equilibrium and Pre-Equlibrium Models for Calculation of Particle Emission Cross Sections and Spectra." (2.24)

HETRICK, D. M., " Tools and Techniques for Evaluation Intercomparison." (2.29)

LARSON, N. M.," "Cross Section Parameterization in the Resolved Resonance Region." (2.25)

PEELLE, R. W., "Covariance Methods for Standards Evaluations." (2.35)

PEELLE, R. W., "The Resolution of Discrepancies Among Nuclear Data." (2.36)

TAGESEN, S., ${ }^{*}$ AND D. C. LARSON, "Approximate Methods for Derivation of Covariance Data." (2.37)

96th Annual Human Factors Society Meeting, Atlanta, GA, October 12-16, 1992; Proc. (1992)

CARTER, R. J., AND J. A. WACHTEL," "Advanced Control Room Design Review Guidelines: Merging Old and New," pp. 423-427. (3.47)

SPELT, P. F., "Human Factors and Safety Issues Associated with Actinide Retrieval from Spent Light Water Reactor Fuel Assemblies," pp. 1158-1162. (3.46)

4th Symposium on Frontiers of Massively Parallel Computing, McLean, VA, October 19-21, 1992; Proc. (1992)

CHOI, J., J. J. DONGARRA, D. W. WALKER AND R. POZO,* "ScaLAPACK: A Scalable Linear Algebra Library for Distributed Memory Concurrent Computers," pp. 120-127. (1.26) 
Oral Presentation, Nuclear Propulsion Technical Interchange Meeting, NASA-Lewis Research Center, Cleveland, OH, October 20-29, 1992

DIFILIPPO, F. C., "Scoping Calculations of Power Sources for NEP."

Eleanor Roosevelt Institute Transcribed Sequences Workshop, San Francisco, CA, October S1-November 1, 1992

MURAL, R. J.,* X. GUAN, J. R. EINSTEIN, AND E. C. UBERBACHER, "Combining Neural Networks and Expert Systems to Identify Features in DNA Sequences." (3.56)

Oral Presentation, Army Research Office Workshop in Modeling and Analysis for Mechanical Systems and Algorithms for Real-Time Vehicle Simulation, Raleigh, NC, November 5-7, 1992

DONGARRA, J. J., "Scalable High-Performance Libraries in Linear Algebra."

1992 American Society of Mechanical Engineers Annual Winter Meeting, Anaheim, CA, November 8-19, 1992; Proc. Fundamentals of Phase Change: Freezing, Melting, and Sublimation, HTD-Vol. 215, P. G. Kroeger and Y. Bayazitoglu, Eds., (1992)

SOLOMON, A. D., ${ }^{*}$ V. ALEXIADES, G. JACOBS, ${ }^{*}$ M. NANEY, ${ }^{*}$ AND M. OLSZEWSKI,* "Latent Heat Thermal Energy Storage for Lunar Oxygen Production," pp. 73-77. (1.61)

4th International Symposium on Robotics and Manufacturing, Santa $\mathrm{Fe}, \mathrm{NM}$, November 11-19, 1992; Proc. Vol. 4 (1992)

PIN, F. G., AND Y. WATANABE,* "Sensor-Based Driving of a Car with Fuzzy Inferencing VLSI Chips and Boards," pp. 613-619. (3.25)

Southeastern Section Meeting of the American Physical Society, Oak Ridge, TN, November 12-14, 1992; Proc. Vol. 97(7) (1992)

ALSMILLER, F. S., R. G. ALSMILLER, JR., AND O. W. HERMANN,* "HECC92, An Updated Intranuclear Cascade Model of High-Energy HadronNuclear Collisions," p. 1673. (4.26)

CARLTON, R. F., ${ }^{*}$ J. A. HARVEY, AND C. H. JOHNSON,"* "R-Matrix Analysis of Neutron Total Cross Section Data in the MeV Region," p. 1676. (2.20)

DICKENS, J. K., AND M. A. MILLER,* "Measurements of ${ }^{181} \mathrm{Ta}(\gamma, x n y p)$ Reactions for Bremsstrahlung Photons Produced by $150-\mathrm{MeV}$ Electrons," p. 1677. (2.3)

HARVEY, J. A., "Neutron Physics at the Oak Ridge National Laboratory," p. 1641. (2.5)

STOCKS, G. M. ** W. A. SHELTON, D. M. NICHOLSON, ${ }^{*}$ G. A. GEIST, AND F. J. PINSKI,* "Fermi Surfaces of $\beta$-Phase NiAl Alloys," p. 1646. (1.17) 
TSVEYBAK, I,* W. BUGG,* J. A. HARVEY, AND J. WALTER,* "Fast Neutron-Induced Changes in Net Impurity Concentration of High-Resistivity Silicon," p. 1675. (2.15)

WESTON, L. W., J. A. HARVEY, AND H. DERRIEN,* "Is the Problem with Pu-239 Fission Cross Section Solved?" p. 1677. (2.16)

American Nuclear Society/European Nuclear Society 1992 International Conference, Chicago, IL, November 15-20, 1992; Trans. Am. Nucl. Soc. 66, (1992)

DICKENS, J. K., R. R. SPENCER, AND J. H. TODD,* "A New Experimental Program of Neutron-Emission Spectral Measurements," p. 475.

HARVEY, J. A., "Historical Perspective on Reactor-Associated Nuclear Data," p. 147.

LARSON, D. C., "Cross Sections of Structural and Shielding Materials," p. 149.

MUCKENTHALER, F. J., "Where Have the Neutrons Gone - A History of the Tower Shielding Facility," p. 427.

PEREZ, R. B., * F. C. DIFILIPPO, "Contributions of Gerard de Saussure to Fission Physics: Subthreshold and Near-Subthreshold Measurements at the ORELA," p. 470.

WESTON, L. W., "Cross Sections of Fissile and Fertile Nuclides," p. 148.

Oral Presentation, IAEA's First Research Coordination Meeting of the Agency's Coordinated Research Program, Debrecen, Hungary, November 17-19, 1992

FU, C. Y., "Resolving the Calculational Differences Among the EFF-2, ENDF/B-VI, and JENDL-3 Evaluations for the ${ }^{58} \mathrm{Ni}(n, \alpha)$ Cross Section."

Oral Presentation, Supercomputing '92, Minneapolis, MN, November 17-20, 1992 GEIST, G. A., "PVM: Portable Distributed Computing Software."

GEIST, G. A., "PVM 3.0 Overview and Tutorial."

GEIST, G. A., "PVM 3.0 Integrating Multiple Paragons into a Virtual Machine."

GEIST, G. A., "PVM 3.0 and the Convex Meta-Series."

Oral Presentation, Fifth ECMWF Workshop on Use of Parallel Processors in Meterology, Reading, United Kingdom, November 23-27, 1992

WALKER, D. W., P. H. WORLEY, AND J. B. DRAKE, "Parallelizing the Spectral Transform Method."

WORLEY, P. H., "The Message Passing Version of the Parallel Community Climate Model." 
Oral Presentation, Department of Electronics and Computer Science, University of Southampton, Southampton, United Kingdom, November 90, 1992

WORLEY, P. H., "PICL: Past, Present, and Future."

Ural Presentation, Mathematics Department, University of Bristol, Bristol, United Kingdom, December 1, 1992

WORLEY, P. H., "Parallelizing Across Time When Solving Time-Dependent Partial Differential Equations."

Oral Presentation, Third Annual Radiation Risk/Safety Technology Transfer Conference, Alexandria, VA, December 1-9, 1992

BURNS, T. J., "Three Dimensional Graphics/Diagnostics System."

JOHNSON, J. O., "MASH Version 1.0 User Instructions and Technology Transfer."

JOHNSON, J. O., T. J. BURNS, J. D. DRISCHLER, R. T. SANTORO, AND J. M. BARNES,* "Armored Vehicle Analyses - U.S. and Foreign Systems."

MORRIS, M. D., "CHERNEM Code: Protracted Dose Mortality."

RHOADES, W. A., "Radiation Calculations in Concrete and Wood-Frame Buildings at Hiroshima and Nagasaki."

SANTORO, R. T., "Overview of the ORNL DNA Radiation Environments Program."

SANTORO, R. T., "Radiation Environments Program," (handouts).

SANTORO, R. T., J. O. JOHNSON, AND S. Y. WHITAKER, " "Two-Meter Box/Phantom Benchmark Experiments, Analyses, and Results."

Oral Presentation, Workshop on-Cluster Computing Supercomputer Computations Research Institute, Florida State University, Tallahassee, FL, December 2-4, 1992

DONGARRA, J. J., "LAPACK for Clusters."

Seminar given at ICASE, NASA Langley Research Center, Hampton, VA, December 10, 1992

SINCOVEC, R. F., "Oak Ridge National Laboratory's Center for Computational Science."

1992 Winter Simulation Conference, Arlington, VA, December 13-16, 1992

MITCHELL, T. J., AND M. D. MORRIS, "The Spatial Correlation Function Approach to Response Surface Estimation." (1.82) 


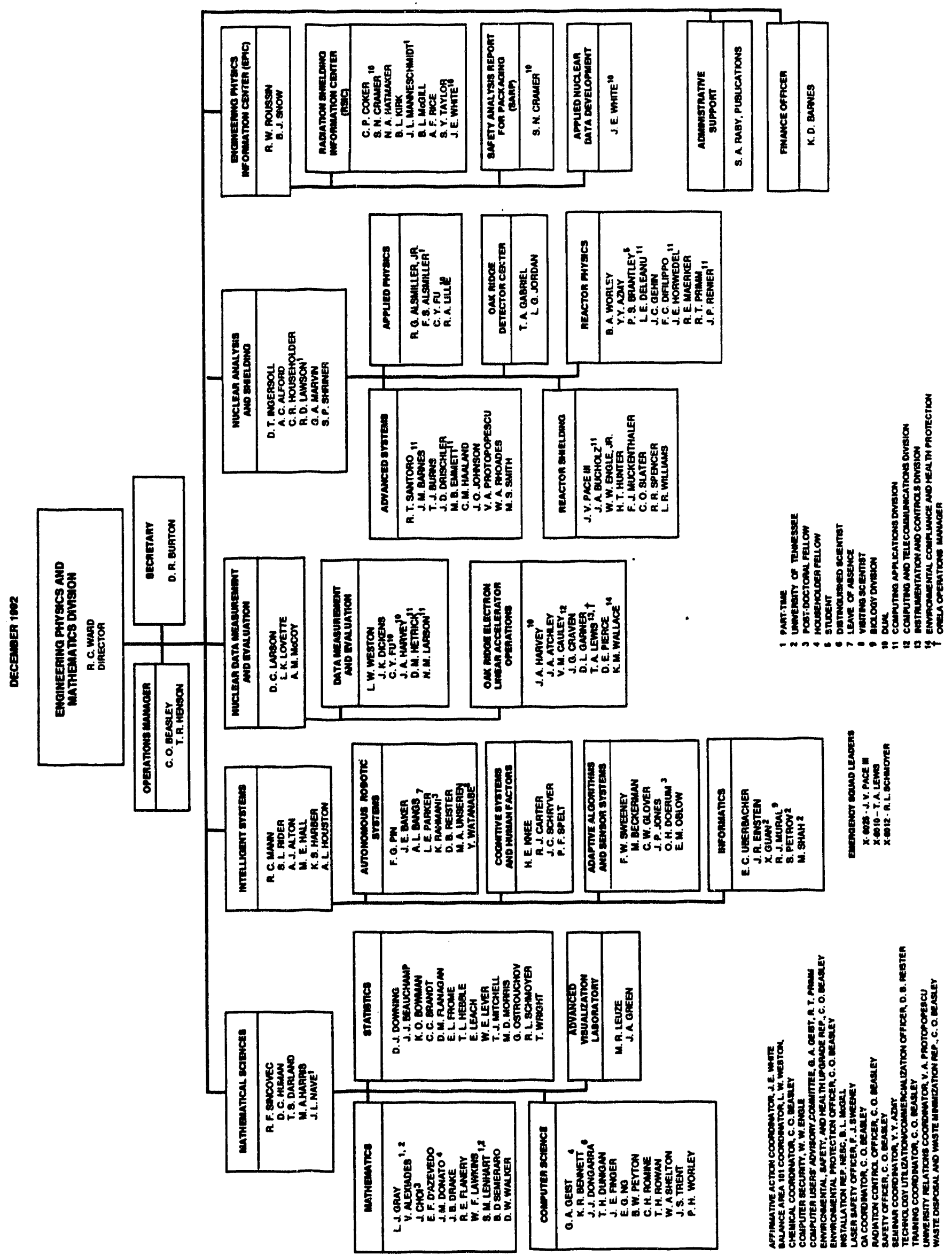




\section{AUTHOR INDEX}

Ables, E. 4.25

Aboughantous, C. 5.6

Alexiades, V. $1.61,1.62$

Alley, G. T. $4.34,4.39$

Alsmiller, F. S. $4.26,4.27,4.28$

Alsmiller, Jr., R. G. 4.1,4.15,4.26,4.27,4.28,4.30, 4.41

Anassontzis, S. 4.29

Andersen, B. L. 3.16

Asgari, M. 5.6

Azmy, Y. Y. 4.58,4.59,4.60,4.61,4.62,4.63,4.67, $4.68,4.69,4.72,4.73,4.75,4.76,4.77,5.1$

Baker, J. E. $3.31,3.32$

Ban, S. 5.7

Bangs, A. L. $3.7,3.12$

Barnes, J. M. 4.43,4.47,4.48,4.49,4.50,4.52

Bauer, M. L. $4.25,4.39$

Bauer, Jr., R. J. 3.40

Beauchamp, J. J. $1.96,1.100$

Beckerman, M. 3.13,3.14,3.33,3.35,3.36,3.37

Beguelin, A. 1.1,1.2,1.3,1.4

Bishop, B. L. $4.25,4.36$

Blair, J. R. S. $1.37,1.38$

Boffi, V. C. 4.73

Botts, J. L. 2.44

Bowman, K. O. $1.85,1.86,1.87,1.88,1.89,1.90,1.92$, 1.94

Brashear, H. R. $4.29,4.34,4.39$

Britton, Jr., C. L. 4.34,4.39

Broadhead, B. L. $2.44,4.18$

Bugg, W. 2.15

Buksa, J. J. 4.23

Burns, T. J. $4.43,4.47,4.51,4.64,4.65,4.66$

Butler, P. L. $3.7,3.8,3.13,3.14$

Carlson, B. M. 1.5

Carlton, R. F. $2.18,2.19,2.20$

Caro, M. 4.2

Carreras, B. A. 1.58

Carter, R. J. $3.42,3.47,3.49$

Cerezo, A. 1.94

Chang, S. J. 1.51

Chatani, K. 4.3

Childs, R. L. 4.53

Chin, P. 1.47

Choi, J. $1.24,1.25,1.26$

Christensen, S. W. 1.100

Cohn, H. O. $4.25,4.39$

Colyer, S. P. 1.102
Coury, B. G. 3.43

Cragle, D. L. 1.111

Cramer, S. N. 2.40,5.2,5.8,5.9

Dale, D. S. 2.26

Davis, S. C. $1.97,1.104$

D'Azevedo, E. F. $1.47,1.48,1.63,1.64,1.70$

Deleanu, L. 4.67

Depiero, F. 3.50

Derrien, H. 2.16

de Saussure, G. 2.21,2.22,2.23

di Brozolo, G. R. 1.28

Dickens, J. K. $2.1,2.2,2.3,2.7,2.8,2.13,2.14,2.41$, $2.42,2.43,2.44$

Difilippo. F. C. $4.2,4.4,4.5,4.6,4.7,4.8,4.9,4.10$, 4.11,4.12,4.13

Dockery, J. 4.78

Dobranich, D. 4.23

Donato, J. M. $1.65,1.66,1.67,1.68$

Dongarra, J. J. 1.1,1.2,1.3,1.4,1.24,1.25,1.26, $1.27,1.28,1.29,1.30,1.31,1.32$

Dowdy, L. W. 1.5

Downing, D. J. $1.110,1.112$

Drake, D. M. 2.26

Drake, J. B. $1.58,1.69,1.74,1.75,1.76,1.77,1.78$

Drischler, J. D. $4.22,4.24,4.43,4.47,4.48,4.49$,

$4.50,4.52,4.56$

Drosg, M. 2.26

Dunford, C. L. 2.32

Dunigan, T. H. $1.6,1.7,1.8,1.9$

Durfee, R. C. 1.112

Edwards, J. J. 1.112

Einstein, J. R. $3.51,3.52,3.53,3.54,3.55$

3.56

Elnawawy, O. A. 4.68

Emery, M. 4.34

Emmett, M. B. 4.54

England, T. R. 2.43

Epperson, S. J. 2.31

Ericison, N. 4.29

Etheridge, J. A. 1.14

Flanagan, D. M. $1.93,1.112$

Flanery, R. E. 1.69

Fletcher, M. C. 1.112

Forsyth, P. A. $1.47,1.48,1.63,1.70$

Foster, I. 1.75

Francis, A. A. 1.98

Frome, E. L. $1.102,1.103,1.111$ 
Fretwurst, E. 4.42

Fu, C. Y. $2.1,2.24,2.27,2.28,2.30,2.31,2.33$, $2.38,4.30,4.33,4.41,5.1$

Fujimura, K. 3.4,3.17,3.18,3.19

Gabriel, T. A. $4.25,4.29,4.30,4.31,4.32,4.33,4.34$, $4.35,4.36,4.37,4.38,4.39,4.41,4.42,5.1$

Gaff, S. J. 4.74

Geist, G. A. $1.1,1.2,1.3,1.4,1.10,1.11,1.12,1.13$ $1.16,1.17,1.18$

Gilbert, J. R. $1.39,1.40$

Ginatempo, B. 1.10

Glover, C. W. $3.27,3.28,3.30,4.33,4.34,4.35$

Goeltz, R. T. 1.112

Gordeev, A. 4.25

Gorman, R. 1.97

Goshaw, A. T. 4.34

Graham, J. 3.9

Graham, P. 3.9

Gray, L. J. $1.49,1.50,1.51,1.52,1.53,1.56$, $1.57,1.59,1.60,1.69$

Guan, X. $3.51,3.523 .53,3.54,3.55,3.56,3.57$

Gupta, A. 1.46

Haaland, C. M. 4.44,4.45,4.46,4.83,4.84

Hack, J. J. 1.77

Haghighat, A. 4.69

Haight, R. C. 2.26

Haire, M. J. 4.14

Hammarling, S. 1.27

Handler, T. $4.32,4.33,4.35,4.36,4.37,4.38,4.42$

Hannan, N. A. 4.23

Harber, K. S. 3.3

Hartley, D. 3.43

Harvey, J. A. $2.4,2.5,2.10,2.11,2.12,2.15$, $2.16,2.18,2.19,2.20,2.22,2.23$

Hasegawa, I. 5.5

Hayashi, M. 4.15

Heath, M. T. 1.14,1.101

Hedge, V. G. 3.9

Hermann, O. W. 4.26,4.27

Hetrick, D. M. $2.1,2.28,2.29,2.30,2.31$

Heywood, T. E. 3.8

Hill, N. W. $2.4,2.10,2.11,2.12,2.19,2.22,2.23$

Hirayama, H. 5.7

Hooper, K. N. 3.43

Hopper, C. M. 4.21

Horwedel, J. E. 4.79,4.80,4.81,4.82

Hu, P. S. $1.97,1.104$

Hunt, G. 4.75

Hyde, J. M. 1.94
Iijima, S. 2.28

Ingersoll, D. T. $4.16,4.17,4.40,4.53,5.4$

Ingersoll, J. K. 4.16

Ingraffea, $\mathbf{A}$. $R$. $1.56,1.57,1.59,1.60$

Iyengar, S. S. 3.9

Jacobs, G. K. $1.61,1.62$

Jager, H. I. 4.81

Jakob; R. 1.77

Jessup, E. R. $1.33,1.34$

Job, P. K. 4.32,4.36,4.38

Johnson, C. H. $2.18,2.19,2.20$

Johnson, J. O. 4.43,4.46,4.47,4.48,4.49, $4.50,4.51,4.52,4.56,4.70$

Johnston, S. E. 3.8

Jolley, R. L. 1.98

Jones, J. P. $3.7,3.8,3.13,3.14$

Jones, T. D. $1.79,1.105,1.106$

Joiner, E. E. 1.102

Kahaner, D. K. 1.35

Kam, F. B. K. 5.6

Kamyshkov, Y. 4.25

Kastenbaum, M. A. $1.85,1.88,1.89,1.90,1.92$

Kehlet, R. A. 4.51

Kerris, K. G. 4.46

Killough, S. M. 3.12,3.15

Kirk, B. L. 4.77,5.1

Klein, J. A. 1.98

Kleinerman, R. A. 1.103

Knee, H. E. 3.42,3.48

Kopecky, J. 2.28

Kraner, H. W. 4.39

Larson, D. C. $2.1,2.6,2.7,2.10,2.13,2.14,2.26$, $2.27,2.28,2.30,2.31,2.32,2.33,2.37,2.38$

Larson, N. M. 2.4,2.10,2.11,2.21,2.22,2.23, $2.25,2.31,2.34$

Lawkins, W. F. 1.71

Laymon, C. M. 2.26

Leboeuf, J. N. 1.58

Lenhart. S. M. $1.54,1.55,1.62$

Leuze, M. R. 1.15

Lever, W. E. 1.110

Liepins, G. E. $3.28,3.38,3.39,3.40,3.41$

Liewer, P. 1.58

Lillie, R. A. 4.1,4.15,4.25,4.54

Lindström, G. 4.42

Littlefield, L. G. $1.102,1.103$

Lutz, E. 1.56,1.57

Lynch, V. E. 1.58 
MacFarlane, R. 5.5

Macklin, R. L. $2.6,2.10$

Macko, K. 4.11

Maerker, R. E. 4.18

Maino, G. 2.28

Manchek, R. 1.1,1.2,1.3,1.4

Mandrekas, J. 4.73

Mann, F. 5.5

Mann, R. C. $3.1,3.4,3.513 .52,3.57$

Manne, L. L. 1.52

Martha, L. F. 1.59

Mattes, M. 5.5

Mays, P. 1.28

McCarthy, J. F. 1.96

McDonnell, J. R. 3.16

McHargue, C. J. 1.110

Miaou, S.-P. $1.97,1.104$

Miller, M. A. 2.2,2.3,2.8

Miller, M. K. $1.86,1.94$

Mitchell, T. J. $1.80,1.81,1.82,1.83,1.84$

Moore, K. 1.3

Moore, T. 4.29

Morgan, G. L. 2.26

Morris, M. D. $1.79,1.80,1.81,1.82,1.83,1.84$, $1.105,1.106$

Moser, D. R. 4.14

Mrochek, J. E. 1.98

Muir, D. 5.5

Mural, R. J. $3.51,3.52,3.53,3.54,3.55,3.56$, 3.57

Naney, M. 1.61

Neis, E. 4.35

Nelson, R. O. 2.7,2.26

$\mathrm{Ng}$, E. G. $1.35,1.39,1.40,1.41,1.42,1.43$, $1.44,1.46$

Nicholson, D. M. 1.17

Nishigori, T. 4.15

Nordborg, C. 2.32

Nyquist, J. E. 1.62

Oblow, E. M. $3.26,3.27,3.28,3.29$

Olszewski, M. 1.61

Osborne-Lee, I. W. 1.98

Ostrouchov, G. 1.95,1.101,1.107,1.108,1.109, 1.112

Ostrouchov, L. S. 1.101

Page, W. C. 3.16

Panini, G. 5.5

Parker, L. E. 3.50

Pattay, R. S. $3.21,3.22,3.24$

Pavlik, A. 2.26
Pawel, J. E. 1.110

Payne, J. S. 1.71

Peelle, R. W. 2.9,2.35,2.36

Perey, C. M. 2.10,2.11

Perey, F. G. $2.10,2.11,2.39,2.40,5.8$

Perez, R. B. 4.12

Perkins, K. R. 4.23

Peyton, B. W. $1.37,1.38,1.40,1.42,1.43,1.44$, $1.45,1.46$

Pin, F. G. $\quad 3.2,3.6,3.9,3.12,3.15,3.16,3.20$,

$3.21,3.22,3.23,3.24,3.25,3.50$

Pinski, F. J. 1.17

Plasil, F. 4.25,4.39

Pothen, A. 1.45

Potyondy, D. O. 1.60

Pozo, R. 1.26

Price, L. E. 4.36,4.37

Primm, III, R. T. $4.19,4.20,4.21,4.67$

Protopopescu, V. $1.54,1.55,4.73,4.74,4.76,4.77$, 4.78

Proudfoot, J. 4.36,4.37,4.38

Railsback, S. F. 4.81

Raman, S. 2.44

Rao, N. S. V. $3.27,3.28,3.30$

Raridon, R. J. 4.82

Rathi, A. K. 1.104

Read, K. 4.25

Reister, D. B. 3.5,3.6,3.14

Renier, J. P. 4.13

Rennich, M. J. 4.25,4.39

Rhoades, W. A. 4.53,4.54

Rice, A. F. 5.11

Riech, V. 4.42

Riehs, P. 2.12

Romana, L. J. 1.110

Rome, J. A. 1.112

Romine, C. H. $1.64,1.101$

Rosenblatt, D. 1.96

Rosener, B. 1.29

Rothberg, E. 1.46

Roussin, R. W. 5.4,5.5,5.11,5.12

Ryan, T. 4.34

Saini, S. 4.35

Sallam, F. 1.102,1.103

Saltmarsh, M. J. 1.112

Saltzen, E. 3.34

San Soucie, C. 1.53

Santoro, R. T. $4.40,4.46,4.51,4.52,4.55,4.56$, 4.57,4.77

Sartori, E. $\quad 5.5,5.12$

Savin, A. 4.25 
Sayer, A. M. 1.103

Schenter, R. E. 2.43

Schiesser, W. E. 1.35

Schmiedmayer, J. $2.12,2.19$

Schmoyer, R. L. 1.91

Schrack, R. A. $2.13,2.14$

Schryver, J. C. 3.48

Shelton, W. A. 1.10,1.16,1.17

Shenton, L. R. $1.85,1.86,1.87,1.88,1.89,1.90$

Shmakov, K. 4.25

Shugart, L. R. 1.96

Shy, C. M. 1.111

Sidani, M. 1.30

Singeltary, B. H. 4.25

Slater, C. O. $4.17,4.71,5.9$

Smirnov, A. 4.25

Smith, E. P. 1.100

Smith, M. S. $4.14,4.22,4.24,4.66$

Smolen, G. R. 4.21

Smyre, J. L. 1.112

Solomon, A. D. $1.61,1.62$

Spelt, P. F. $3.44,3.45,3.46$

Steiner, R. 4.41

Stocks, G. M. $1 \cdot 10,1 \cdot 17$

Stojanovic, S. 1.55

Storjohann, K. 3.34

Sunderam, V. S. $1.1,1.2,1.3,1.4,1.12,1.13$ 1.18

Swarztrauber, P. N. 1.77

Sweeney, F. J. 3.13,3.14,3.35

Symon, J. $3.22,3.24$

Tagesen, S. $2.26,2.37$

Takano, H. 5.5

Tang, W.-P. $1.47,1.48,1.63,1.70$

Tankersley, W. 1.111

Tarkovsky, E. 4.25

Taylor, J. R. 3.20

Terranova, M. 3.43

Thompson, S. 1.35

Todd, J. F. $2.1,2.13,2.14,2.17$

Todd, R. A. 4.25,4.39

Trubey, D. K. $\quad 5.3,5.10$

Tsveybak, I. 2.15

Uberbacher, E. C. $3.51,3.52,3.53,3.54,3.55,3.56$, 3.57

Unseren, M. A. 3.4,3.10,3.11

Uppuluri, V. R. R. 3.29

van de Geijn, R. A. 1.31,1.32

Vandergriff, D. 4.34

Vasseur, H. A. 3.20
Vineyard, T. A. 1.112

Vonach, H. K. $2.26,2.28$

Vose, M. D. $3.39,3.41$

Wachtel, J. A. 3.47

Wade, R. 1.3

Wagner, T. D. 1.5

Wagschal, J. J. 4.18

Walker, D. W. $1.19,1.24,1.25,1.26,1.32,1.58,1.72$, $1.73,1.74,1.76$

Walker, R. L. 2.44

Walter, J. 2.15

Walton, J. T. 4.23

Wang, L. W. 3.38

Ward, R. C. 1.15

Wasson, O. A. $2.13,2.14$

Watanabe, H. $3.22,3.23,3.24,3.25$

Wender, S. A. $2.7,2.26$

Wert, J. J. 1.110

Weston, L. W. 2.16,2.17

Whitaker, S. Y. 4.57

White, J. E. 5.4,5.5,5.6

Wilkinson, J. H. 1.27

Williams, M. L. 5.6

Williamson, D. L. $1.75,1.77$

Wing, S. 1.111

Wing, W. R. 1.112

Wintenberg, A. 4.29

Winters, R. R. 2.19

Wolf, S. 1.111

Wood, D. H. $4.22,4.24$

Wood, J. L. 1.111

Worley, B. A. $4.13,4.23$

Worley, P. H. $1.5,1.20,1.21,1.22,1.23,1.36,1.74$, $1.76,1.78$

Wright, R. Q. $4.17,4.82,5.4,5.6$

Wright, T. $1.97,1.98,1.99,1.104$

Wu, W. 3.30

Yager, R. 4.78

Yamamuro, N. 2.28

Ylvisaker, D. 1.84

Young, K. G. 4.25

Young, P. G. 2.26

Young, R. W. 1.79,1.106

Yuan, X. 1.45

Zeitnitz, C. 4.42

Zhao, Z. $2.38,2.39$

Zhixiang, Z. 2.27 
INTERNAL DISTRIBUTION

1. V. A. Alexiades

2. A. C. Alford

3. R. G. Alsmiller, Jr.

4. A. J. Alton

5. B. R. Appleton

6. D. K. Arakawa

7. Y. Y. Azmy

8. J. B. Ball

9. J. M. Barnes

10. K. D. Barnes

11. C. Beasley

12. J. J. Beauchamp

13. M. Beckerman

14. B. A. Berves

15. R. G. Bilbrey

16. K. O. Bowman

17. C. C. Brandt

18. D. R. Burton

19. R. J. Carter

20. R. L. Childs

21. D. F. Craig

22. S. N. Cramer

23. T. S. Darland

24. E. F. D'Azevedo

25. L. Deleanu

26. J. K. Dickens

27. F. C. Difilippo

28. J. M. Donato

29. J. J. Dongarra

30. D. J. Downing

31. J. B. Drake

32. T. H. Dunigan

33. J. R. Einstein

34. M. B. Emmett

35. W. W. Engle

36. R. Fenstermaker

37. R. E. Flanery

38. E. L. Frome

39. C. Y. Fu

40. T. A. Gabriel

41. D. L. Garner

42. G. A. Geist

43. R. K. Genung

44. L. J. Gray

45. C. M. Haaland

46. W. R. Hamel

47. M. A. Harris

48. F. C. Hartman

49. N. A. Hatmaker

50. R. B. Honea

51. C. R. Householder

52. H. T. Hunter

53. D. T. Ingersoll

54. J. O. Johnson

55. F. B. Kam

56. B. L. Kirk
57. H. E. Knee

58. D. C. Larson

59. N. M. Larson

60. R. D. Lawson

61. E. Leach

62. R. A. Lillie

63. L. K. Lovette

64. R. E. Maerker

65. R. C. Mann

66. G. A. Marvin

67. D. W. McDonald

68. B. L. McGill

69. T. J. Mitchell

70. R. M. Moon

71. M. D. Morris

72. F. J. Muckenthaler

73. R. J. Mural

74. J. L. Nave

75. E. G. $\mathrm{Ng}$

76. E. M. Oblow

77. C. E. Oliver

78. G. Ostrouchov

79. J. V. Pace, III

80. B. W. Peyton

81. F. G. Pin

82. M. L. Poutsma

83. R. T. Primm, III

84. V. A. Protopopescu

85. C. E. Pugh

86. S. A. Raby

87. D. E. Reichle

88. D. B. Reister

89. A. F. Rice

90. C. R. Richmond

91. S. L. Rider

92. C. H. Romine

93. R. W. Roussin

94. T. H. Rowan

95. R. T. Santoro

96. R. L. Schmoyer

97. J. C. Schyrver

98. J. Sheffield

99. S. P. Shriner

100. W. D. Shults

101. C. S. Sims

102. R. Sincovec

103. C. O. Slater

104. M. S. Smith

105. B. J. Snow

106. P. F. Spelt

107. R. R. Spencer

108. J. O. Stiegler

109. A. W. Trivelpiece

110. E. C. Uberbacher

111. M. A. Unseren

112. R. C. Ward 
113. C. West

114. J. E. White

115. L. R. Williams

116. B. A. Worley

117. P. H. Worley

118. T. Wright

119. R. W. Brockett (Consultant)

120. D. J. Dudziak (Consultant)

121. J. E. Leiss (Consultant)
122. N. Moray (Consultant)

123. M. F. Wheeler (Consultant)

124-148. EPMD Reports Office

149. Laboratory Records - RC

150-151. Laboratory Records

152. Central Research Library

153. Document Ref. Section

154. ORNL Patent Office

\section{EXTERNAL DISTRIBUTION}

155. Office of the Assistant Manager for Energy Research \& Development, DOE-ORO Oak Ridge, TN 37830

156-157 Office of Scientific \& Technical Information, P.O. Box 62, Oak Ridge, TN 37831

158. Dr. Jim Beall, ER-72, Office of Health and Environmental Research, U.S. Department of Energy, Germantown, Washington, DC 20585

159. Sam E. Berk, Office of Fusion Energy, ER-533, GTN, J-216, U.S. Department of Energy, Germantown, MD 20874

160. Kathleen C. Bernard, 513 Princess St., Alexandria, VA 22314

161. T. A. Callcott, Director, The Science Alliance Program, 53 Turner House, University of Tennessee, Knoxville, TN 37996

162. John Conway, Mathematics Department, University of Tennessee, Knoxville, TN 37996-1300

163. Jim Cook, Westinghouse Savannah River Company, P.O. Box 616, Building 704-Z, Aiken, SC 29802

164. G. D'Alessio, Director, Research and Technology Development Division, DP-242, GTN-A-383, U.S. Department of Energy, Germantown, MD 20874

165. M. Dougherty, NASA Lewis Research Center, 21000 Brookpark Road, MS SVR, Cleveland, OH 44135

166. Doug Halford, Oak Ridge National Laboratory, Grand Junction Office, P.O. Box 2567, Grand Junction, CO 81502

167. Dr. Anton J. Haug, Manager, ASW Advanced Requirements, Martin Marietta Aerospace, 103 Chesapeake Park Plaza, Baltimore, MD 21220

168. P. B. Hemmig, Safety and Physics Branch, Office of Technology Support Programs, U.S. Department of Energy, Washington, DC 20585

169. Dr. Dan Hitchcock, Office of Scientific Computing, ER-7, Applied Mathematical Sciences, Office of Energy Research, U.S. Department of Energy, Washington, DC 20585

170. Dr. Fred Howes, Office of Scientific Computing, ER-7, Applied Mathematical Sciences, Office of Energy Research, U.S. Department of Energy, Washington, DC 20585

171. Dr. Thomas R. James, Director, ITER \& Technology Division, Office of Fusion Energy, U.S. Department of Energy, Washington, DC 20585

172. Dr. Gary Johnson, Office of Scientific Computing, ER-7, Applied Mathematical siciences, Office of Energy Research, U.S. Department of Energy, Washington, DC 20585

173. R. A. Kehlet, Defense Nuclear Agency, 6801 Telegraph Rd., Alexandria, VA 22310

174. M. K. Kerris, Army Research Laboratory, 2800 Powder Mill Rd., Adelphi, MD 20783

175. Dr. T. A. Kitchens, Office of Deputy Assistant Secretary for International Affairs, ER-7, GTN, G-218 U.S. Department of Energy, Germantown, MD 20874

176. Milt Klein, Electric Power Research Institute, 3412 Hillview Avenue, P.O. Box 10412, Palo Alto, CA 94303

177. Joel L. Kristal, Director, Program Operations Support Division, EM-333 TREV, 307, 12800 Middlebrook Road, U.S. Department of Energy, Germantown, MD 20874

178. R. M. Loesch, Assistant Secretary for Environment, Safety and Health, EH-411, GTN, G-137, U.S. Department of Energy, Germantown, MD 20874

179. F. C. Maienschein, 838 West Outer Drive, Oak Ridge, TN 37830

180. Dr. Richard A. Meyer, Division of Nuclear Physics, ER-23, GTN, U.S. Department of Energy, Washington, DC 20585

181. William Lake, Civilian Radioactive Waste Management, RW-431, FORS-7F-043, U.S. Department of Energy, Washington, DC 20585

182. Paul B. Moyers, Office of Energy Research, AD-611, FORS, 1G-079, U.S. Department of Energy, Washington, DC 20585 
183. Dr. David Nelson, Director of Scientific Computing, ER-7, Applied Mathematical Sciences, Office of Energy Research, U.S. Department of Energy, Washington, DC 20585

184. J. Poore, Computer Science Department, University of Tennessee, Knoxville, TN 37996-1300

185. R. E. Stephens, Director, Office of University and Science Education Programs, ST-50, FORS, 3F-043, U.S. Department of Energy, Washington, DC 20585

186. B. H. Thomas, Office of Nonproliferation Policy, AN-20, FORS, GA-017, U.S. Department of Energy, Washington, DC 20585

187. C. Ward, Foreign Science and Technology Center, 220 7th St. N.E., Charlottesville, VA 229015396

188. Dr. Pete M. Williams, Office of Advanced Reactor Programs, U.S. Department of Energy, 19901 Germantown Road, Germantown, MD 20874

189-213. Given Engineering Physics and Mathematics Division Special Distribution AS 

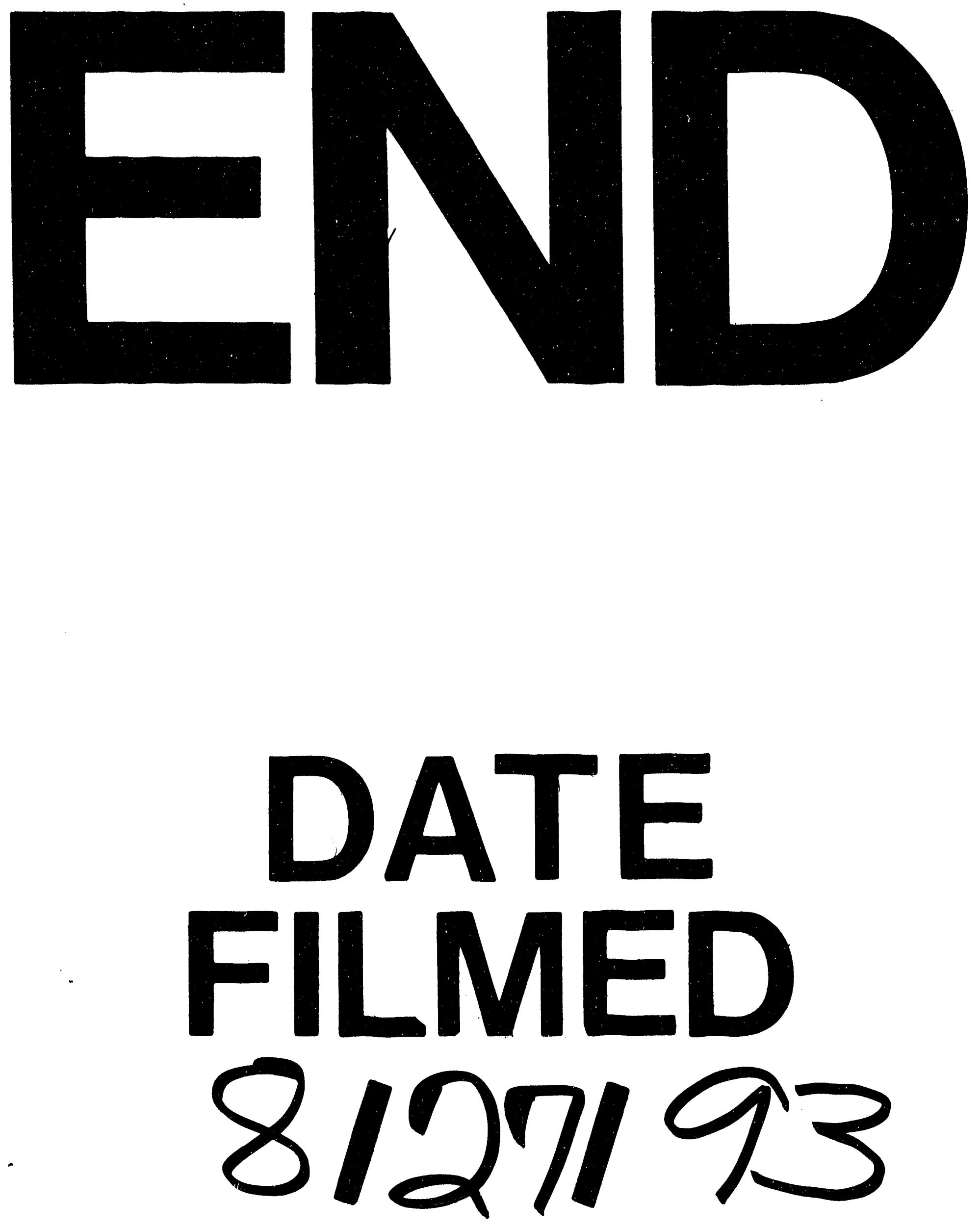